SPACE-CHARGE LIMITS ON THE TRANSPORT OF ION BEAMS IN A LONG ALTERNATING GRAOIENT SYSTEM*

Michael G. Tiefenback

Lawrence Berkeley Laboratory

University of California

Berkeley, CA 94720

November 1986

* Tiis work was supported by the Office of Energy Research, Office of Basir. Energy Sciences, U.S. Department of Energy, under Contract No. DE-ALD3-76SF DOO98. 


\section{LEGAL NOTICE}

This book was prepared as an account of work sponsored by an agency of the lonited states Covernment. Veither the l'niled States Covern. ment nor any agessy thereof, nor any of their employecs, makes aty warranny, express or intplied or assumes amy lexal liability or responsibitity. ferr the accuracy. completeness. or usefulnew if any information, apparaties, product, or process disclosent, or represents that its ase wiould nut infringe pritately owned rights. Heference herein

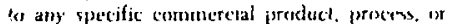
serice by trader marne, trademark, thantıfacturer. or otherwise, does rot necessarily constitute os imply its endorsement, recommendation, or favoring by the United States Covernment or any agency thereof. The views and opinions of authors expressed herein do not necessarily state or reflect those of the United States Government or any agency thereof. 


\title{
Space-Charge Limits on the Transport of Ion Beams in a Long Alternating Gradient System
}

\author{
Michael G. Tiefenback
}

\begin{abstract}
We have experimentally studied the space-charge-dominated transport of ion beams in an alternating-gradient channel, without acceleration. We parameterize the focusing strength in terms of the zero-current "betatron" oscillation phase advance rate, $\sigma_{0}$ (degrees per focusing period). We have investigated the conditions for "stability", defined as the constancy of the total current and phase space area of the beam during transport. We find that the beam may be transported with neither loss of current nor growth in phase area if $\sigma_{0}<90^{\circ}$. In this regime, the space-charge repulsive force can counter $98-99 \%$ of the externally applied focusing field, and the oscillation frequency of the beam particles can be depressed by self-forces to almost a factor of 10 below the zero-current value, limited only by the optical quality of our ion source. For $\sigma_{0}>90^{\circ}$, we find that collective interactions bound the maintainable density of the beam, and we present a simple, semi-empirical characterization for stability, within our ability to distinguish the growth rate from zero in our apparatus. Our channel comprises 87 quadrupole lenses, 5 of which are used to prepare the beam for injection into the non-azimuthallysymmetric focusing channel.
\end{abstract}




\section{Acknowledgements}

As John Donne has said in a different context, "No man is an island." Similarly, no thesis can be completed without the help and encouragement of many people. I take this opportunity to thank, in public, my in-all-ways wonderful wife, Claudia J. Tiefenback, for her patient and loving support throughout this part of our life together.

To all of the people who helped and guided me in researching this thesis, I would like to give public acknowledgement. The following list is incomplete by its very nature, and I apologize to all who are not, but should have been, included. I thank Dr. D. L. Judd, who introduced me to the subject of particle accelerators. I thank Dr. D. Keefe, who served as my advisor at Lawrence Berkeley Laboratory, and Professor W. B. Kunkel, who served as my faculty advisor from the University of California at Berkeley.

I would like to thank A. Faltens for many hours of discussion on the nature of things, and for providing much in the way of physical insight into the workings of accelerator hardware. I greatly appreciate the work of Drs. L. J. Laslett, C. Kim, and J. Shiloh, who designed and organized much of the hardware for the Single Beam Transport Experiment. Dr. WN. Chupp provided me with an introduction to vacuum science, as well as discussions on other topics of value. Mr. Bill Ghiorso and Dr. A. A. Warwick did much of the work on the data collection system used in the experiments of this thesis.

All of the technical support staff of the accelerator design group at Lawrence Berkeley Laboratory are due recognition for their contributions to the design, fabrication, and construction of the Single Beam Transport Experiment, and I would like to single out for particular recognition D. Vanecek, R. Hipple, C. Pike, and G. Stoker.

Finally, I would like to thank Drs. G. Krafft and M. C. Lampel for their 
friendship and for long hours of discussion while we were all graduate sti lents together. 
Contents

1 Introduction 1

1.1 Approaches to Fusion $\ldots \ldots \ldots \ldots \ldots$

1.2 Heavy Ion Fusion $\ldots \ldots \ldots \ldots \ldots \ldots$

1.3 Early Related Work ... . . . . . . . . . . 7

1.4 Recent results relevant to heavy ion fusion . . . . . . 7

1.5 Summary of Goals and Results . . . . . . . . . . 10

2 Beam Dynamics $\quad 12$

2.1 Conventions . . . . . . . . . . . . 12

2.2 Envelope Descriptions . . . . . . . . . . 13

2.2.1 Beam with negligible space-charge ......... 13

2.2.2 Beam with linear space-charge field included . . . 15

2.2.3 RMS envelope description including nonlinear spacecharge ................... 18

2.3 Bore Requirements as a Function of Lens Strength . . . . . 19

3 Experimental Hardware 21

3.1 Vacuum System . . . . . . . . . . . . . 21

3.2 Ion Source and Injector Assembly . . . . . . . . . 24

3.3 Marx generator . . . . . . . . . . . . . 27

3.4 Quadrupoles \& Power Supplies . . . . . . . . . 27

3.5 Diagnostics ....................... 31

3.5.1 Multiwire Profile Monitor . . . . . . . . . . 31

3.5.2 Beam current monitors . . . . . . . . . 35

3.5.3 Phase space diagnostics $\ldots \ldots \ldots \ldots$ 
3.5.4 Diagnostic positioning .............. 38

3.5.5 Attenuator Wheel .............. 41

3.6 Data Acquisition System . . . . . . . . . . . . 41

4 Lattice Performance and Data Analysis 45

4.1 Injector Perveance and Performance . . . . . . . . . 45

4.2 Particle Energy and Lattice Strength Calibration . . . . . . 47

4.3 Beam Matching Procedures . . . . . . . . . . . . . 50

4.4 Emittance Measurements ................ 53

4.5 Data Analysis ........................ 53

4.5.1 Stability Criterion ............... 54

4.5.2 Phase space data presentation .......... 54

4.5.3 Intensity parameterization and $\sigma$ values ....... 55

5 Beam Measurement Results

5.1 Results for $\sigma_{0} \leq 88^{\circ} \ldots \ldots \ldots$

5.1.1 Focusing aberrations .............. 57

5.1.2 Beam loss bounds ............... 59

5.1.3 Beam envelope measurements ........... 63

5.1.4 Phase Space Measurement Results ... . . . . . . 67

5.2 Discussion of Results for $\sigma_{0} \leq 88^{\circ} \ldots \ldots . \ldots . . \ldots 9$

5.2.1 Low-emittance limits . . . . . . . . . . . . 69

5.2.2 Plasma-like behavior .............. 73

5.2 .3 Dependence on source distribution ......... 76

5.3 Results for $\sigma_{0} \geq 88^{\circ} \ldots \ldots \ldots 77$

5.3.1 Envelope measurements ............. 80

5.3.2 Phase space measurement results . . . . . . . . 81 
5.4 Discussion for $\sigma_{0} \sim 90^{\circ} \ldots \ldots \ldots \ldots \ldots \ldots \ldots 10 \ldots \ldots$

5.5 Discussion for $\sigma_{0}$ much greater than $90^{\circ} \ldots \ldots \ldots \ldots$

5.6 Summary of Results . . . . . . . . . . . . . 112

6 Other Experiments $\quad 117$

6.1 Space-charge Coupling of Transverse Dimensions . . . . . 117

6.2 Image Charge Effects . . . . . . . . . . . . 121

6.2.1 Shift in the coherent oscillation frequency . . . . . 121

6.2.2 Emittance growth for off-axis beams . . . . . . 125

6.3 Background Gas Stripping of $\mathrm{Cs}^{+} \ldots \ldots \ldots \ldots \ldots$

7 Error Analysis $\quad 135$

7.1 Errors in determination of $\sigma_{0} \ldots \ldots \ldots \ldots \ldots$

7.2 Incomplete scans of phase space . . . . . . . . . 136

7.3 Beam end-effects . . . . . . . . . . . . . 141

7.4 Effect of non-zero slit size $\ldots \ldots \ldots \ldots \ldots 141$

7.5 slit misalignment . . . . . . . . . . . . 143

7.6 Space charge effects $\ldots \ldots \ldots \ldots \ldots \ldots \ldots$

7.7 Slit-beam perturbation . . . . . . . . . . . 144

7.8 Pulse-to-pulse variation of the beam . . . . . . . 145

7.9 Background gas effects . . . . . . . . . . . . 145

7.10 Secondary electron yield variations $\ldots \ldots \ldots \ldots \ldots$

7.11 Error summary . . . . . . . . . . . . . 146

$\begin{array}{ll}\text { A Beam Dynamics } & 148\end{array}$

A.1 Space-Charge Dominated Transport . . . . . . . . . 148

A.2 Useful Approximate Calculations . . . . . . . . . . 150 
B Faraday Cups $\quad 154$

B.1 Secondary Electron Yields . . . . . . . . . . . . . . . 154

B.2 Slit Cups ..................... 154

B.3 Absolute Current Measurement . . . . . . . . . . . 155

B.3.1 Shallow Faraday Cups . . . . . . . . . . 155

B.3.2 Deep Faraday Cups .............. 157

B.3.3 Secondary Ion Effects .............. 159

C Emittance Increase due to Grids in the Beam Path 160

C.1 Mechanism ...................... 161

C.2 Termination oi the Accelerating Field of the Injector . . . . . 164

C.3 Effect of Multiple Biased Grids . . . . . . . . . . . . 165

$\begin{array}{ll}\text { D Quadrupole Design } & 168\end{array}$

E Ion Sources $\quad 175$

E.1 Source Recoating Procedure . . . . . . . . . . . . 175

E.2 Performance. . . . . . . . . . . . . 177

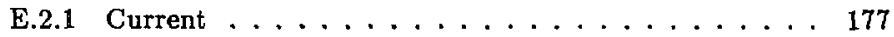

E.2.2 Source Emittance . . . . . . . . . . . . 177

E.2.3 Beam Purity ................. 177 
List of Figures

3.1. Physical layout of the SBTE lattice and diagnostics. . . . . 22

3.2 SBTE experimental apparatus view . . . . . . . . 23

3.3 SBTE injector schematic drawing . . . . . . . . 25

3.4 SBTE injectcr exploded view . . . . . . . . . 26

3.5 SBTE Marx generator circuit schematic . . . . . . . 28

3.6 Interior of one of the SBTE vacuum tanks with quadrupole array installed. . . . . . . . . . . . . 32

3.7 SBTE quadrupole power supply schematic . . . . . . . 33

3.8 Follow-and-hold circuit diagram for profile monitors . . . . 34

3.9 Shallow Faraday cup diagram . . . . . . . . . 36

3.10 Deep Faraday cup diagram . . . . . . . . . . 37

3.11 Principle of the two-slit emittance measurement . . . . . . 39

3.12 Slit collector assembly . . . . . . . . . . . 40

3.13 Diagnostic wheel assembly . . . . . . . . . . . 42

3.14 HP-85 based data acquisition system layout and flow chart . 43

4.1 Comparison of measured and calculated injector performance 48

4.2 Direct measurement of $\sigma_{0} \ldots \ldots \ldots \ldots \ldots \ldots$

5.1 SBTE injector current density profile . . . . . . . 58

5.2 Focusing aberrations at matching section exit . . . . . 60

5.3 Faraday cup current comparison along lattice . . . . . . 62

5.4 Higher order beam oscillations shown in beam profiles .... 65

5.5 Measured second and third moments of beam along lattice for $\sigma_{0}=83^{\circ} . \ldots \ldots \ldots \ldots \ldots \ldots \ldots \ldots \ldots \ldots \ldots$ 
vini

5.6 Plot of : vs. $\epsilon(i)$ for $15 \mathrm{~mA}, \sigma_{0}=59^{\circ}$. . . . . . . . 68

5.7 Plots of $i$ vs. $\epsilon(i)$ for $15 \mathrm{~mA}, \sigma_{0}=78^{\circ}, \ldots . . . . .70$

5.8 Plots of $i$ vs. $\epsilon(i)$ for $15 \mathrm{~mA}, \sigma_{0}=83^{\circ} \ldots \ldots 71$

5.9 Current transmitted through the lattice for $\sigma_{0} \sim 90^{\circ} \ldots 72$

5.10 Comparison between Debye length and beam sheaths . . . . 75

5.11 Overall stability summary, with envelope instability loundary 79

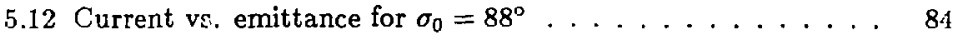

5.13 Phase siace contours for $\sigma_{0}=88^{\circ} \ldots \ldots . \ldots . \ldots 5$

5.14 Current vs. emittance for $\sigma_{0}=98^{\circ} \ldots \ldots . \ldots . \ldots 6$

5.15 Phase space contours for $\sigma_{0}=98^{\circ} \ldots \ldots$. . . . . . . 87

5.16 Current vs. emittance for $\sigma_{0}=102^{\circ} \ldots \ldots$. . . . . . . 89

5.17 Phase space contours for $\sigma_{0}=102^{\circ} \ldots \ldots . . . \ldots 9$

5.18 Threshold measurement for $\sigma_{0}=118^{\circ} \ldots \ldots$. . . . . . . 93

5.19 Threshold measurement for $\sigma_{0}=124^{\circ} \ldots \ldots . . \ldots 5$

5.20 Current vs. emittance for $\sigma_{0}=124^{\circ} \ldots \ldots$. . . . . . . . $\$ 6$

5.21 Phase space contours for $\sigma_{0}=124^{\circ} \ldots \ldots$. . . . . . . 97

5.22 Current vs. emittance for $\sigma_{0}=134^{\circ} \ldots \ldots . . \ldots 9$

5.23 Phase space contours for $\sigma_{0}=134^{\circ}$. . . . . . . . . . 100

5.24 Current vs. emittance fớr $\sigma_{0}=145^{\circ} \ldots \ldots$. . . . . . . . 102

5.25 Phase space contours for $\sigma_{0}=145^{\circ} \ldots \ldots . . \ldots 103$

5.26 Comparison of output beams for $\sigma_{0}=145^{\circ}$ for $10 \mathrm{~mA}$ and 34 . $\mathrm{mA}$ injection ...................... 104

5.27 Maxirnum growth rates for envelope instability . . . . . . 106

5.28 Current vs. emittance for $\sigma_{0}=94^{\circ}$, unstable beam. . . . . 107

5.29 Phase space contours for $\sigma_{0}=94^{\circ}$, unstable beam. . . . . 108

5.30 Estimated on-axis current density along stability boundary. . 113 
5.31 Result summary, presented in terms of $\sigma / \sigma_{0} . \ldots \ldots \ldots 114$

6.1 Equilibration of the emittances in the $x$ and $y$ planes. . . . 119

6.2 Contour plots for horizontal-vertical equilibration. . . . . 120

6.3 Model treatment of induced charge as due a line image. . . . 123

6.4 Beam centroid vs. Jattice strength for high-current beam . . . 124

6.5 Simulation results for high-current beam offcenter in lattice . 126

6.6 Measured emittance growth for high-current, off-center beam 128

6.7 Current vs. fressure in SBTE for $120 \mathrm{keV}$ particle energy. . . 131

6.8 Current vs. pressure in SBTE for $160 \mathrm{keV}$ particle energy . . 132

7.1 Emittance error mechanism due to high beam divergence or misalignment. . . . . . . . . . . . . . . . 139

B.1 Current response of the $\mathrm{SFC} \ldots \ldots \ldots \ldots \ldots$

B.2 DFC bias response curves $\ldots \ldots \ldots \ldots \ldots \ldots$

C.1 Transverse electric field near grid wires . . . . . . . 162

C.2 Grid spoiling of the emittance compared to calculated values. 167

D.1 SBTE electrostatic quadrupole dimensions. . . . . . 169

D.2 Transverse field linearity of the SBTE periodic lattice. . . . 172

D.3 Variation of $\sigma_{0}$ in the SBTE with particle oscillation amplitude. . . . . . . . . . . . . . . . . 173 
List of Tables

4.1 Generalized perveances for SBTE and for two proposed accelerators . . . . . . . . . . . . . . . . 46

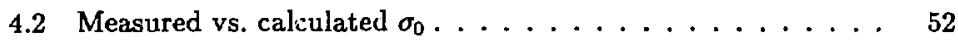

5.1 Summary of output beam parameters, calculated and measured $\ldots \ldots \ldots \ldots \ldots \ldots \ldots \ldots \ldots$

6.1 Measured cross-sections for charge-changing collisions for 120 and $160 \mathrm{keV} \mathrm{Cs}{ }^{+}$ions against air. . . . . . . . . 133

7.1 Summary of error bounds . . . . . . . . . . . 147

A.1 Envelope eqn. solutions compared against transfer matrix with smoothed space-charge . . . . . . . . . . . . . . 151

C.1 Variation of the grid perturbation of the emittance with grid structure .................... 163

C.2 Intrinsic emittance of gun before grid passage . . . . . . 165

C.3 Relative effect of various terminating grids vs. calculation . . 165

D.1 Coefficients used in field expansion $\ldots \ldots \ldots \ldots \ldots$

E.1 Distribution of light ion impurities $\ldots \ldots \ldots \ldots 179$ 


\section{Chapter 1: Introduction}

Nuclei of light atoms can fuse together into heavier nuclei, with the accompanying release of up to about $0.4 \%$ of their rest mass energy, depending on the reactants and products. To adapt this process for practical power generation requires confining the very hot reactants until a large fraction of the fuel has fused. Three distinct means are known for maintaining the fuel at high density and fusion temperatures for long enough for a large proportional "burn" - gravitational confinement, magnetic confinement, and what is called "inertial confinemeni."

Inertial confinement fusion, or ICF, is a generalization of the hydrogen bomb mechanism. This process has been under development for application to sub-milligram fuel pellets since about 1960 [1], using lasers or beams of light charged particles rather than a fission bomb to drive the fuel to the conditions required for nuclear fusion. In addition, in 1974, Masclke [2] proposed that beams of heavy ions with parameters appropricte to pellet. fusion requirements might be generated using the existing particle accelerator technology developed for high-energy particle physics research. A major extrapolation of existing technology is necessary; for this application, it is necessary to transport beams with currents in the multi-kiloampere range over long distances, while maintaining a low transwerse beam temperature.

The goal of this thesis work was to determine the maximum brightness and average current density maintainable for a space-charge dominated beam in a long focusing channel. This subject is not well-treated in the conventional accelerator literature, because effects other than intrinsic space-charge dominated beam interactions with the focusing structure have limited the intensity of the beam. Circular accelerators, for example, may nat have space-charge forces of more than a few percent of the average external focusing forces. 
The reason is that the nonlinear space-charge force increases the spread in the transverse oscillation frequency of the particles. As a result, some particles become resonant with the unavoidable errors in the focusing channel $[3,4,5]$. Conventional linear accelerators (linacs) have used radio-frequency cavity acceleration and have been current-limited by, for example, the particle source current or space-charge forces in the low-velocity segment of the accelerator. For these and other reasons, alternating gradient focusing has not hitherto been explored for space-charge dominated beams. The heavy ion induction linac approach to inertial confinement fusion (ICF), however, centers about space-charge dominated transport of high-brightness beams, and the practical limitations are of overriding interest $[6,7]$.

\subsection{Approaches to Fusion}

The fusion reaction requiring the least energy to initiate is the deuteriumtritium reaction

$$
{ }^{2} \mathrm{H}+{ }^{3} \mathrm{H} \rightarrow{ }^{4} \mathrm{He}+\mathrm{n}+17.6 \mathrm{MeV},
$$

requiring a minimum temperature of several $\mathrm{keV}$, and reaching the peak reaction rate (assuming Maxwellian velocity discributions for the reactants) at about $50 \mathrm{keV}$. Lawson [8] quantified the requirements for density and confinement time for thermal distributions, obtaining the result called the Lawson criterion: for the output of fusion energy from the confined fuel greater than that required to heat the fuel and overcome radiative losses, the product of the number density, $n$, and the confinement time (for the energy, rather than the particles), $\tau$, must satisfy

$$
n \tau \geq 10^{14} \mathrm{sec} \mathrm{cm}^{-3},
$$

for the reaction between deuterium and tritium, at a temperature above 10 $\mathrm{keV}$. For other reactants, the temperature and $n \tau$ product required are larger. 
Of the confinement mechanisms listed above, gravitational confinement is clearly unworkable on a terrestrial scale. In order to confine a fusion plasina by gravitational self-attraction, a mass of the order of the solar mass is required, and the resulting star is not a useful example.

Magnetic confinement schemes attempt to impede the free loss of fuel by applying a magnetic feld within the confinement region. The presence of the magnetic field has no effect on the ultimate thermal equilibrium state of a system [9]. The purpose of the field is to slow the rate of approach to equilibrium and greatly increase the plasma confinement time. Work in this field is ongoing and has resulted in confinement times and temperatures within about an order of magnitude of what is required for "scientific breakeven," or the release of fusion energy equal to the energy used to confine and heat the plasma.

Inertial confinement derives its name from two inertial effects important in pellet fusion. A mass of fuel is accelerated radially inward to a high velocity, on the order of $2 \times 10^{7} \mathrm{~cm} / \mathrm{sec}[6]$. The inertia of the fuel carries it inward, $o$ rarcuming the increasing pressure of the material and drastically increasing the density of the fuel. In pellet applications this radial acceleration is driven by heat ablation of the outer layers of the pellet. The input energy is supplied by an external driver, with the compressional force supplied by the rocket-like ablation of the heated outer material of the pellet.

The second inertial effect limits the disassembly of the dense fuel mass. The fuel particles have an average velocity deterr:ined by the temperature of the fuel. The fuel cannot escape from the high-density region in a time much shorter than that given by the ratio of the pellet radius to the thermal velocity of the ions, even in the case of a transparent plasma. However, the plasma is quite opaque to the ions, even at a temperature of $20 \mathrm{keV}$. For 1 
mg of D-T compressed into a sphere $0.1 \mathrm{~mm}$ in radius, with a temperature of $20 \mathrm{keV}$, the $90^{\circ}$ scattering length is on the order of $0.001 \mathrm{~mm}$. Because the density is proportional to $R^{-3}$, where $R$ is the minimum radius of the pellet, and the disassembly time $\tau$ is roughly proportional to $R$, the product of the disassembly time and the compressed fuel density is proportional to $R^{-2}$ for a fixed fuel temperature. If the density is made sufficiently high and the temperature of the fuel is raised to about $20 \mathrm{keV}$, then the fuel simply cannot escape until a large fraction has had time to undergo fusion.

The earliest work on laser-driven ICF was directed toward the use of very small pellets, to provide a means of laboratory simulation of nuclear weapons dynamics. The initial estimates of 1-10 kJ driver energies proved to be much too low, and much of the material about the project was declassified. The possibility of applying this idea to commercial generation of electrical power was recognized, and experimental programs for both electron and light-ion beams as drivers for ICF were initiated, using the pulse power technology from flash radiography and nuclear weapons effects simulation programs. Then, in 1974, Maschke [2] proposed adapting existing particle accelerator technology to the generation of high-current, high power, heavy ion beams for use as an ICF friver (heavy ion fusion, or HIF). Recent estimates of the required pellet size and driver energy and power lie in the range of $1 \mathrm{mg}$ of D-T mixture and 3-5 M.J of driver beam energy, with peak power in the hundreds of terawitts [i 0,11 .

\subsection{Heavy Ion Fusion}

Hereafter we will consider only particle-beam driven ICF, and will show some advantages offered by a heavy ion beam as an ICF driver as opposed to a light particle beam. The physical properties of matter dictate certain parameters for ICF, in addition to the obvious requirement that the beam 
be focused onto the pellet [6]:

- In order to obtain the required force to drive the implosion, there must be a minimum specific energy deposition (joules/gm) in the ablative portion of the pellet, on the order of $20 \mathrm{MJ} / \mathrm{gm}$. This will raise the temperature of the ablative material to about $200 \mathrm{eV}$. The requirement provides an upper bound on the allowable range of the primary beam particles.

- The fuel compression must be nearly adiabatic to maximize the fuel density attained before incoming shock waves heat the core of the pellet and initiate thermonuclear reactioss. In addition to requiring that the energy from the primary beam be deposited in the outer layer of the pellet, there must be no transport processes active to carry energy into the pellet core and increase the pressure forces opposing the implosion.

- The beam power must be high enough to deliver the energy on the time scale of the pellet implosion. To deliver megajoules of energy on the time scale of $10 \mathrm{~ns}$ to a pellet of surface area about $0.1 \mathrm{~cm}^{2}$ requires power levels of $10^{14}$ watts and power densities of $10^{15}$ watts $/ \mathrm{cm}^{2}$.

These considerations constrain the range (in $\mathrm{g} / \mathrm{cm}^{2}$ ) of the beam particles and thus limit the particle energy, while at the same time requiring very high beam power and total energy. For electrons or low-Z nuclei, the low energy per particle required by the range limitation necessitates very large electrical current, and total charge in the beam $\left(\sim 1 \mathrm{MeV}\right.$ and $10^{8}$ amperes for electrons and $\sim 5 \mathrm{MeV}$ and $2 \times 10^{7}$ amperes for protons). To reduce the technical problems of transport and focusing as much as possible, Maschke [2] suggested that heavy ion beams with parameters almost within reach of current accelerator technology might be used. Beam parameters for ions 
of atomic weight near 200 are expected to be $10 \mathrm{GeV}$ per nucleus, with a peak beam current of 10 kiloamperes and a total charge corresponding to about 300 particle microcoulombs $(\mu \mathrm{C})$. Although there are several unsoived problems with this approach, none presently seems prohibitive $[12,13,10,11]$. While these extremes of particle energy, total current, and total energy are not currently available simultaneously, beam parameters of this order are found in different accelerators around the world. The Bevalac complex at Berkeley, California, routinely accelerates such heavy nuclei as uranium to this energy, although the number of particles per pulse corresponds only about $10^{-3}-10^{-2}$ particle $\mu \mathrm{C}$. In the ATA electron induction linear accelerator at Lawrence Livermore Laboratory, beam currents of several tens of $\mathrm{kA}$ are attained with relativistic electrons. Beams with a total energy of several MJ are manipulated routinely within the ISR complex at CERN and at Fermilab in the USA.

Practical considerations of reaction energy deposition and wall damage [12] dictate a standoff distance of about 5-10 meters from the reactor wall and final beam lenses to the pellet. In order to focus the beam from this distance orito a pellet of radius a few mm, the optical quality of the beam must be very high. Additionally, cost and efficiency concerns require the average current density along the accelerator to be as high as possible without heating the beam transversely enough to hinder final focusing of the beam onto the pellet. This regime of near-laminar flow, space-charge dominated beam transport has until recently been treated only theoretically $[14,15,16,17]$, with no body of experimental results to provide a comparison. How intense may a beam be before the very large electrical potential energy collectively couples to the random transverse particle kinetic energy and heats the beam ? The research reported here is driven by the requirement to transport as much current as possible in each beam, subject to being able to focus the beam onto a pellet, 
and to take advantage of the higher acceleration efficiency of induction linacs as the beam current becomes higher.

\subsection{Early Related Work}

An early study of the transport of high-current electron beams with low emittance was made by Brewer [18] in connection with traveling wave electron tube amplifiers. His experiment used solenoid focusing, and he found he could not avoid beam loss between cathode and anods unless the magnetic field strength was raised to about 1.2 times the value theoretically required for a cold beam. The cold-beam ideal case for this transport is called Brillouin flow [19], for which the current density and confining field are related (nonrelativistically) by

$$
j=B_{x}^{2} \frac{\epsilon_{0} q v_{x}}{2 m}
$$

in mks units, where $j$ is the current density, $B_{s}$ is the solenoid field strength, $q$ is the particle electric charge, and $m$ is the particle mass.

In detailed experiments, Brewer established that his electron beam contained a significant component of thermally hot particles, which explained the need for the higher field. The particles with high transverse veiocities (due to aberrations at the cathode edge) Brewer called "translaminar." The question of the long-term stability of the transport of cold, high-cu:rent beams, particularly in an alternating gradient (A.G.) focusing array, had not been addressed before the interest in the topic for heavy ion fusion (HIF) brought it to the forefront of attention $[20,17,21]$.

\subsection{Recent results relevant to heavy ion fusion}

Based on materials limits and attainable magnetic focusing strengths, Maschke [22] proposed a limit on the beam power transportable in an alter- 
nating gradient channel

$$
P=C\left(\frac{A}{Z}\right)^{4 / 3} B_{Q}^{2 / 3}\left(\beta_{\mathrm{r}} \gamma\right)^{5 / 3} \epsilon_{\mathrm{N}}^{2 / 3}(\gamma-1)
$$

where $P$ is in watts, $A$ is the atomic number of the ion, $Z$ is its charge state in units of the electron charge, $B_{Q}$ is the magnetic field strength at the pole-tips of the focusing magnets, $\epsilon_{\mathrm{N}}$ is the normalized emittance (see section 2.2.1), $\beta_{r}$ and $\gamma$ are the usual relativistic factors, and $C$ is a constant. Under certain assumptions, discussed below, Maschke showed that the value assumed by the constant is

$$
C \simeq 1.67 \times 10^{15} \mathrm{Watt} /(\text { Tesla-meter })^{2 / 3}
$$

The focusing of the channel must counter both the space-charge defocusing of the beam and the spread in transverse velocities of the beam particles. The space-charge forces cancel part of the applied lens fields, leaving only part of the vacuum focusing field to contain the thermal motion of the particles. The value of the constant in the above equation is dependent on the allowable relative strengths of the channel focusing and the space-charge self-defocusing of the beam. Maschke estimated, based on his experience with radio-frequency linear accelerators, that only about half of the average restoring force provided by a focusing channel could be canceled by the space-charge self-defocusing of the beam without enabling collective interactions which would degrade the optical quality of the beam. Taking the average focusing of the channel to be linear, a single particle passing along the channel executes harmonic oscillations in the transverse dimensions, which may be characterized by a "wave number," or phase advance rate for the oscillation. This is often quoted in terms of a parameter, $\sigma_{0}$, in degrees of phase advance per period of the (periodic) focusing structure (see Ch. 2). 
For a given particle mass and velocity along the cliannel, $\sigma_{0}$ is proportional to the square root of the equivalent spring constant of the restoring well. For a beam with non-negligible space-charge forces, the overall restoring force is weakened, and the individual particles execute harmonic oscillations at a lower phase advance rate denoted by $\sigma$. If only a factor of two decrease in the overall focusing were possible without degrading the optical quality of the beam, then we would be constrained to have $\sigma / \sigma_{0} \geq 0.7$.

Gluckstern [15] had already published a calculation for a model beam distribution (Kapchinskij-Vladimirskij, or K-V; see section 2.2.2) in the limit of a uniform focusing latîice which indicated the possibility of unstable beam behavior from purely transverse effects. This was later extended to periodic focusing structures by Smith and co-workers [16], and indicated that serious difficulties might arise if the channel focusing were raised to $\sigma_{0}>60^{\circ}$ (see section 2.2.1). Results published from this work by Hofmann, Laslett, Smith, and Haber [20] show many isolated patches and extended regions of instability for the $K-V$ beam distribution.

Numerical simulation work has confirmed some of the analytical results for the $\mathrm{K}-\mathrm{V}$ distribution (the only analytically known equilibrium distribution for a non-uniform focusing channel). In addition, simulation work is not restricted to this distribution, and has shown that many of the instabilities of the analytical work saturate with little or no practical effect on the beam.

The overall conclusion reached from this theoretical work was that the focusing strength of the lattice should be limited to $\sigma_{0}<60^{\circ}$ per focusing period in an aiternating gradient channel, and the space-charge forces should not reduce the depressed phase advance, $\sigma$, below about $24^{\circ}$.

There have been experimental results elsewhere $[23,24]$, as well as from this work [25], which have shown that within the limits of the lengtlis of the 
respective channels, the space-charge forces may be so great as to almost totally cancel the external focusing. In this case, the beam temperature is so low as to be almost totally negligible, and the particle flow in the beam is nearly laminar (a particle will drift from one side of the beam to the other only after traveling through many periods of the focusing channel.

\subsection{Summary of Goals and Results}

For a fusion pellet driver, the emittance (see section 2.2.1) must be held below certain bounds, given by the requirement to focus the beam onto a fusion fuel pellet. The major result of this work is the measurement of the threshold for collective degradation of the emittance in high space-charge beam environmenis, providing information both for accelerator design in the heavy ion fusion program and for theoretical efforts to interpret the mechanism of such collective effects. To give an experimental answer to the question of beam stability we constructed a focusing channel for transport of a coasting beam (constant particle energy downstream of the beam injector). We measured the beam emittance at injection, exit, and intermediate lattice locations, to determine the low-emittance limits on the stable transport of high-current beams, resulting from collective space-charge interaction with the focusing channel. We varied the relative contributions of the space-charge and emittance terms of the envelope equations (section 2.2.2) both by attenuating the beam and by raising the transverse emittance using a set of biasable grids we could insert into the beam. We define the beam to be "stable" if the output current and emittarce are equal to the values at injection.

Our results show no observable lower bound to the emittance maintainable for a given beam current (for 40 periods of quadrupole transport), as long as the focusing channel has a zero-current phase advance per period, $\sigma_{0}$, less than about $90^{\circ}$. This result has important consequences for the design of 
HIF drivers.

For $v_{0}>90^{\circ}$, there is a band of instability in the envelope equations themselves. While we observed a definite lower bound on the emittance for $\sigma_{0}>90^{\circ}$ (where the envelope equations have the possibility of being unstable), the empirical limit was not weil-correlated with this lowest-order instability band. Instead, the limit on beam intensity was well-described for $\sigma_{0}$ as high as $140^{\circ}$ by a relation of the form of the smooth approximation relationship (derived assuming a $\mathrm{K}-\mathrm{V}$ distribution for the beam)

$$
\sigma^{2}=\sigma_{0}^{2}-\frac{1}{2}\left(\frac{180^{\circ}}{\pi} \frac{2 L}{v_{x}} \omega_{p}\right)^{2}
$$

with a constant limiting $\omega_{\mathrm{p}}$ given by

$$
\omega_{\mathrm{p}} \frac{2 L}{v_{s}} \simeq \frac{2 \pi}{3}
$$

Here, $\sigma$ is the phase advance of the beam particles inciuding space-charge effects, $\omega_{p}$ is the usual (ion) plasma frequency, $v_{x}$ the beam velocity along the channel, and $2 L$ is the period of the focusing channel. This value for $\omega_{\mathrm{p}}$ corresponds to one "plasma oscillation" occurring while the beam transits three focusing periods.

Thus the boundary $\sigma_{0} \simeq 90^{\circ}$ separates a region of parameter space in which the beam emittance grows very rapidly (on a length scale of ten focusing periods) from a region giving no noticeable growth $(<10 \%)$ in our experimental channel of over 40 focusing periods. 


\section{Chipter 2: Beam Dynamics}

\subsection{Conventions}

We use right-handed $(x, y, z)$ coordinates for a linear transport system in which $x$ is positive in the vertically upward direction and $z$ is positive in the direction of the beam. We will use nonrelativistic formulae, relativistic corrections being negligible throughout this work. There is often confusion of the relativistic "beta", the ratio of a velocity to the speed of light, with the "beia functiosi" of accelerator physics, to be defined shortly. We will denote the relativistic quantity by $\beta_{r}=v / c$, all other occurrences of $\beta$ being the accelerator function, which depends on the distance along the focusing channel. All external focusing fields wili be assumed linear in transverse displacement, with a givan perindicity iu $z$. We will denote derivatives with respect to $z$ by primes. Thus, for example, $x^{\prime} \equiv \partial x / \partial z$. Finally, we will denote the RMS values of a parameter, sucls as the beam offset in the $I$ dimension, by a iilde, as

$$
\tilde{x} \equiv \sqrt{\left((x-\bar{x})^{2}\right)}
$$

Space-charge will be assumed to be the cnly source of non-linear fields, and for most of this work orly the linear part of this $f_{e}-1$ is conside ed. We will assume a monoenergetic longitudinal beam distribution without acceleration. The experimental apparatus incorporates electrostatic quadrupoles for beam focusing, and we will write all focusing fields as electric fields, using mks units. Recall that the magnetic equivalent involves substitution of $\underline{\mathbf{v}} \times \underline{\mathbf{B}}$ for $\underline{\mathbf{E}}$. The space-charge field will be calculated locally as if it were purely transverse, that is, neglecting beam envelope variațions.

Periodic focusing systems are well-covered in the classic paper by Courant and Snyder [26], which includes the limitations placed on circular machines 
by lens errors. A very comprehensive treatment of accelerators with good mathematical background material is given by Brück [27]. Some additional material on space-charge-dominated transport is included in Appendix A.

\subsection{Envelope Descriptions}

\subsubsection{Beam with negligible space-charge}

In a periodic focusing channel with a restoring force linear in the transverse displacements $x$ and $y$, the equations of motion for a particle may be written (neglecting self-fields) as

$$
\begin{aligned}
& x^{\prime \prime}(z)+K_{x}(z) x(z)=0 \\
& y^{\prime \prime}(z)+K_{y}(z) y(z)=0,
\end{aligned}
$$

where $K_{x}$ and $K_{y}$ are periodic functions of $z$. For continuous solenoid focusing $K_{x}(z)=K_{y}(z)=$ constant.; for an A.G. system $K_{x}(z)=-K_{y}(z)=K(z)$. Unless otherwise stated, we will assume henceforth that the focusing is from a quadrupole array, so that the force constants in the $x$ and $y$ planes differ only in sign. We will assume that the focusing array has the focus-drift-defocusdrift (FODO) geometry, in which the lens fields reverse sign with each lens along the array.

From Eqns. 2.1 one obtains the equations for the beam envelopes $u(z)$ and $b(z)$ in the $x$ and $y$ planes, respectively, (as in Lawson [28], for example)

$$
\begin{aligned}
& a^{\prime \prime}(z)+K(z) a(z)-\frac{\epsilon_{z}^{2}}{a^{3}(z)}=0 \\
& b^{\prime \prime}(z)-K(z) b(z)-\frac{\epsilon_{y}^{2}}{b^{3}(z)}=0
\end{aligned}
$$

where $\epsilon_{x}$ and $\epsilon_{y}$ are the "emittances" for the two planes, defined below. 
In an electrostatic system, the focusing field coefficient $K(z)$ is given by

$$
K(z)=\frac{q}{m v_{x}^{2}} \frac{\partial \mathrm{E}_{x}}{\partial x}
$$

which altemates in sign from lens to lens as $E_{x}$ changes sign. Here $m$ is the particle mass, $q$ its charge, and $v_{z}$ the $z$-velocity. It is conventional in the field of accelerator physics to call the $\left(x, x^{\prime}\right)$ space "phase space," even though in mechanics that term is usually reserved for the space described by the canonically conjugate variables $\left(x, p_{x}\right)$. (We will neglect all vector potential effects, so that $p_{x}$ is purely the mechanical momentum.) The area occupied by the beam in $\left(x, z^{\prime}\right)$ space is $\pi$ times the product of the semiaxes of the ellipse. The product of the semi-axes of the ellipse is denoted by $\epsilon$, and so the area of the beam in phase space is $\pi \epsilon$. When quoted in the (scaled) canonical phase space $\left(x, p_{x} / m c\right)$, the area is $\pi \epsilon_{\mathrm{N}}$. The quantity $\epsilon$ is ralled the "emittance," or "unnormalized emittance," and is the quantity occurring in the envelope equations. The quantity $\epsilon_{\mathbb{N}}=\epsilon \beta_{r} \gamma$, where $\beta_{r}$ and $\gamma$ are the nsual relativistic factors, is called the "normalized emittance." It is particularly useful because it remains constant upon acceleration of the beam, in the absence of nonlinear forces.

The "acceptance" is the transverse phase space area into which particles may be injected without subsequent loss to the walls. A beam is called "matched" in a periodic lattice if its envelope has the same periodicity as the lattice. The envelope of a mismatched beam undergoes oscillations about the matched solution, requiring a larger aperture for the same beam emittance.

The motion of individual particles in the $x$ plane can be written

$$
x(z)=\sqrt{\beta(z) C} \sin \left\{\psi(z)+\psi_{0}\right\}
$$

where $\psi(z)$ is called the betatron phase function, $\beta(z)$ is called the envelope 
function, and $\mathcal{C}$ and $\psi_{0}$ are constants deperding upen the initial conditions. The particle motion is broadly sinusoidal ("betatron oscillations") with a superposed higher frequency flutter component described by $\sqrt{\beta}$. The futter occurs as a particle is alternately focused and defocused by the lenses. The average betatron motion is due to the average restoring force of the A.G. channel.

For any given beam particle undergoing linear focusing, there is a constant of the motion,

$$
\left(\frac{x}{\beta}\right)^{2}+\left(x^{\prime}-\frac{\beta^{\prime}}{2 \beta} x\right)^{2}=C
$$

where $\beta$ is the envelope function defined above. For the outermost particles in phase space of a matched beam, $C=\epsilon$. The largest offset in $x$ of any particle is given by $\sqrt{\beta \epsilon}$, so this quantity gives the radius of the beam. The particle oscillation frequency in linear accelerators is usually characterized by $\sigma_{0}$ in units of degrees of phase advance per period (analogous to a wave-number), often loosely called the zero-current "tune" of the lattice.

The individual particle motion we have been describing to this point is referred to as "incoherent." In addition one can have a "coherent" motion of the beam about the axis if the beam is misaligned. In this case the centrcid of the beam oscillates about the axis with the betatron wave-number $\sigma_{0}$, as if the beam were a macroparticle.

\subsubsection{Beam with linear space-charge field included}

In the case of azimuthally symmetric focusing, uniform in $z$, many distributions which include self-fields in a self-consistent way may be written either explicitly or inplicitly [29], because the total energy is a constant of the motion. However, only one of these, called the "Kapchinskij-V)adimirskij" or "K-V" distribution [30], is known to be generalizable to the case of periodic 
focusing, for which the transverse Hamiltonian is not a constant of the motion. The $\mathrm{K}-\mathrm{V}$ distribution is a microcanonical distribution in terms of a modified transverse Hamiltonian, based on the constant of the motion given in Eqn. 2.4. That is, all the beam particles lie on a single surface in the total transverse phase space $\left(x, x^{\prime}, y, y^{\prime}\right)$. This particular distribution function results in a uniform particle density within an elliptical boundary in $(x, y)$ space. This gives rise to linear space-charge fields, satisfying the requirements for Eqn. 2.4 to give a constant of the motion. The envelope equations for the two transverse planes are coupled by the self-field, although the emittances $\epsilon_{x}$ and $\epsilon_{y}$ ideally are independent constants. Denoting the beam radius in the $x$ plane by $a(z)$ and in the $y$ plane by $b(z)$, we add the defocusing space-charge term to Eqns. 2.2 and obtain the K-V envelope equations

$$
\begin{aligned}
& a^{\prime \prime}(z)+K(z) a(z)-\frac{Q}{a(z)+b(z)}-\frac{\epsilon_{z}^{2}}{a^{3}(z)}=0 \\
& b^{\prime \prime}(z)-K(z) b(z)-\frac{Q}{a(z)+b(z)}-\frac{\epsilon_{y}^{2}}{b^{3}(z)}=0
\end{aligned}
$$

where $Q=\frac{q I}{\pi \epsilon_{0} m v_{x}^{3}}$ in the nonrelativ istic limit. We calculate the matched envelope parameters for our experiment by direct integration of these equations, using the focusing fiela renresentation given in Appendix D.

Within this linear field model, a useful relation between the values of $\epsilon$, $\sigma_{0}$, and the space-charge depressed "tune", $\sigma$, may be found for a uniform focusing channel. In this case, $a=b$ and $K$ is constant with no sign difference between the dimensions, and we have

$$
a^{\prime \prime}(z)+K a(z)-\frac{Q}{2 a(z)}-\frac{\epsilon^{2}}{a^{3}(z)}=0 .
$$

The matched beam envelope in a uniform focusing channel is constant, which 
we will denote by $R$. The trajectory equation for the particles in the matched beam is

$$
x^{\prime \prime}(z)+K x(z)-\frac{Q}{2 R^{2}} x(z)=0 .
$$

We see that the zero-current wave number $\kappa_{0}$ for the particle oscillation is given by $\kappa_{0}^{2}=K$, and the space-charge depressed value $\kappa$ is given by

$$
\kappa^{7}=\kappa_{0}^{2}-Q / 2 R^{2}
$$

If we further note that the value for $\epsilon$ is given by the product of the beam radius and the maximum crossing angle of particles at the beam axis, $\epsilon=R^{2} \kappa$, then we may obtain the relation

$$
\kappa^{2}+\frac{Q}{2 \epsilon} \kappa-\kappa_{\mathrm{C}}^{2}=0
$$

We use this result to obtain the proportionality (written in the periodic channel notation given above)

$$
\sigma \propto \frac{\epsilon}{I}\left(\sigma_{0}^{2}-\sigma^{2}\right)
$$

This is approximately valid for $\sigma_{0}<90^{\circ}$, and implies that if $\sigma$ much less than $\sigma_{0}$, then $\sigma \propto(\epsilon / I) \sigma_{0}^{2}$. If $\sigma_{0}$ and the current are constant, then $\sigma$ increases monotonically with $\epsilon$.

By perturbing Eqn. 2.6, we may calculate the frequency of envelope oscillations for the envelope mode in which the two dimensions oscillate in-phase. Denoting the perturbation by $\delta$, we obtain

$$
\delta^{\prime \prime}+\left(K+\frac{Q}{2 R^{2}}+3 \frac{\epsilon^{2}}{R^{4}}\right) \delta=0
$$

We eliminate $\epsilon$ in favor of the matched beam radius $R$ by using Eqn. 2.6. By using the expressions for $\kappa_{0}$ and $\kappa$ above we obtain the envelope frequency $k$, 
using the periodic channel notation,

$$
k^{2}=2 \sigma_{0}^{2}+2 \sigma^{2}
$$

This mode frequency will be used in Ch. 5. In the zero-current limit, $\sigma \rightarrow \sigma_{0}$, and so $k \rightarrow 2 \sigma_{0}$. This is because when the particles have executed half of a betatron oscillation, the envelope has executed one full oscillation. The high-current envelope oscillation frequency could have been written in terms of the plasma frequency, showing explicitly the space-charge-dominated nature of the mode in that limit. We have used the channel strength parameter $\sigma_{0}$, linearly related to the cold-beam plasma frequency, as may be seen from Eqn. 2.7 by rewriting $Q / R^{2}$ in terms of $\omega_{p}^{2}$.

\subsubsection{RMS envelope description including nonlinear space-charge}

An alternative envelope analysis has been given by Sacherer [31] and Lapostolle [14] in terms of various moments of the trajectory equation, averaged over an arbitrary beam distribution. The resulting hierarchy of coupled moment equations is examined for a low-order quantity which can be approximated from other considerations to close the chain of variables and equations. The unnormalized RMS emittance is such a quantity, formally defined by

$$
\epsilon_{\mathrm{RMS}} \equiv \sqrt{\left\langle(x-\bar{x})^{2}\right\rangle\left\langle\left(x^{\prime}-\overline{x^{\prime}}\right)^{2}\right\rangle-\left\langle(x-\bar{x})\left(x^{\prime}-\overline{x^{\prime}}\right)\right\rangle^{2}},
$$

where both the brackets $\rangle$ and overlines denote an average over the distribution. The nonlinear portion of the total field drives the $z$ variation of the RMS emittance. The equations for the RMS radius of the beam in terms of the linearized self-field and the RMS emittance are identical in form to the $\mathrm{K}-\mathrm{V}$ equations (Eqns. 2.5) with the restriction that the beam distribution in $(x, y)$ space must have elliptical symmetry. The RMS emittance is either taken to be constant or is approximated in other simple ways from known 
behavior. For any beam with constant RMS emittance, the RMS beam envelope is well-modeled by the envelope equations. For a $\mathrm{K}-\mathrm{V}$ distribution the usual radius is equal to $2 \tilde{x}$, where $\tilde{x}$ is the RMS beam radius, and the usual emittance, $\epsilon$, is equal to $4 \epsilon_{R M S}$. We will identify $2 \tilde{x}$ with the beam radius calculated from the envelope equations (Eqns. 2.5), and will use $4 \epsilon_{\mathrm{RMS}}$ for the beam emittance.

\subsection{Bore Requirements as a Function of Lens Strength}

A desirable property of an accelerator for many applications, including HIF, is that the average current density over the bore of the accelerator be high. For a single beam of fixed current, this primarily involves minimizing the maximum beam radius, although for multiple beams the packing fraction for the beams within the bore is also importani.The relationship between lattice strength and envelope size differs considerably between emittancedominated and current-dominated beam transport, as we will now illustrate using the thin-lens quadrupole lattice. Because of the symmetry, the maximum in the beam radius occurs in the focusing lens. For a thin-lens FODO lattice with focusing period $2 L$, lens focal length $\pm f$, and no space-charge, we obtain

$$
\beta_{\max }=2 L \frac{1+\sin \left(\sigma_{0} / 2\right)}{\sin \sigma_{0}},
$$

where $\sin \left(\sigma_{0} / 2\right)=L / 2 f$. Note the divergence of $\beta_{\max }$ as $\sigma_{0}$ approaches $180^{\circ}$, for which the particle becomes resonant with the focusing lattice. For given values of $\epsilon$ and $L$, the required beam aperture is minimized (for the thin lens model) for $\sigma_{0} \simeq 76.3^{\circ}$.

In contrast, for an ideal zero-emittance beam with non-zero current, the minimum in required aperture occurs at a lens strength well beyond the $\sigma_{0}=$ $180^{\circ}$ limit for zero-current beams (see Appendix A). The beam itself, viewed 
as a macroparticle, will become resonant with the focusing if $\sigma_{0}$ is raised too high, even though the individual particles experience a much weaker overall focusing and do not become resonant with the external focusing as in the zero-current case. This provides a great incentive to determine the strongest lattice usable for high current transport. For low emittance, in the absence of collective instability, and with perfect alignment of the lattice and beam, very high intensity beams could be transported. The different response of the envelope to lens strength for emittance-dominated and for current-dominated beams is due to the much different dependence on the beam radius of the space-charge defocusing term and the emittance term in the envelope equations, Eqns. 2.5. 


\section{Chapter 3: Experimental Hardware}

The Single Beam Transport Experiment (SBTE) consists of

- a vacuum enclosure with pumps,

- an ion source and high voltage power supply,

- an array of five independently powered quadrupoles, M1-M5, to enable the beam to be matched from the source into the transport lattice,

- eighty-two quadrupoles, $Q 1$ through $Q 82$, in a periodic focusing lattice with their power supplies

- several types of diagnostic hardware, and

- an automated data acquisition system.

A sketch of the overall system layout is included in Fig. 3.1.

\subsection{Vacuum System}

The vacuum tanks were made of aluminum, seven in number, exclusive of the source chamber. The tanks housing the transport channel were 31 inches in height and 27 inches in width, measured internally. The one adjoining the source was made 5.5 feet in length, with the other six being six feet in length. The large transverse dimensions allowed for the possible inclusion of a cryopumping panel. Access ports were provided every 6 inches to permit almost any conceivable diagnostic configuration (see Fig. 3.2). Five Balzers turbopumps, model T'P-510, each with a net pumping speed (after mounting on adapter flanges) of about $350 \mathrm{l} / \mathrm{sec}$, were distributed along the beamline. Each tank was vacuum baked to about $120^{\circ} \mathrm{C}$, attaining an ultimate pressure with one pump of $2-3 \times 10^{-8}$ Torr, as indicated by ion gauge. The bakeout 


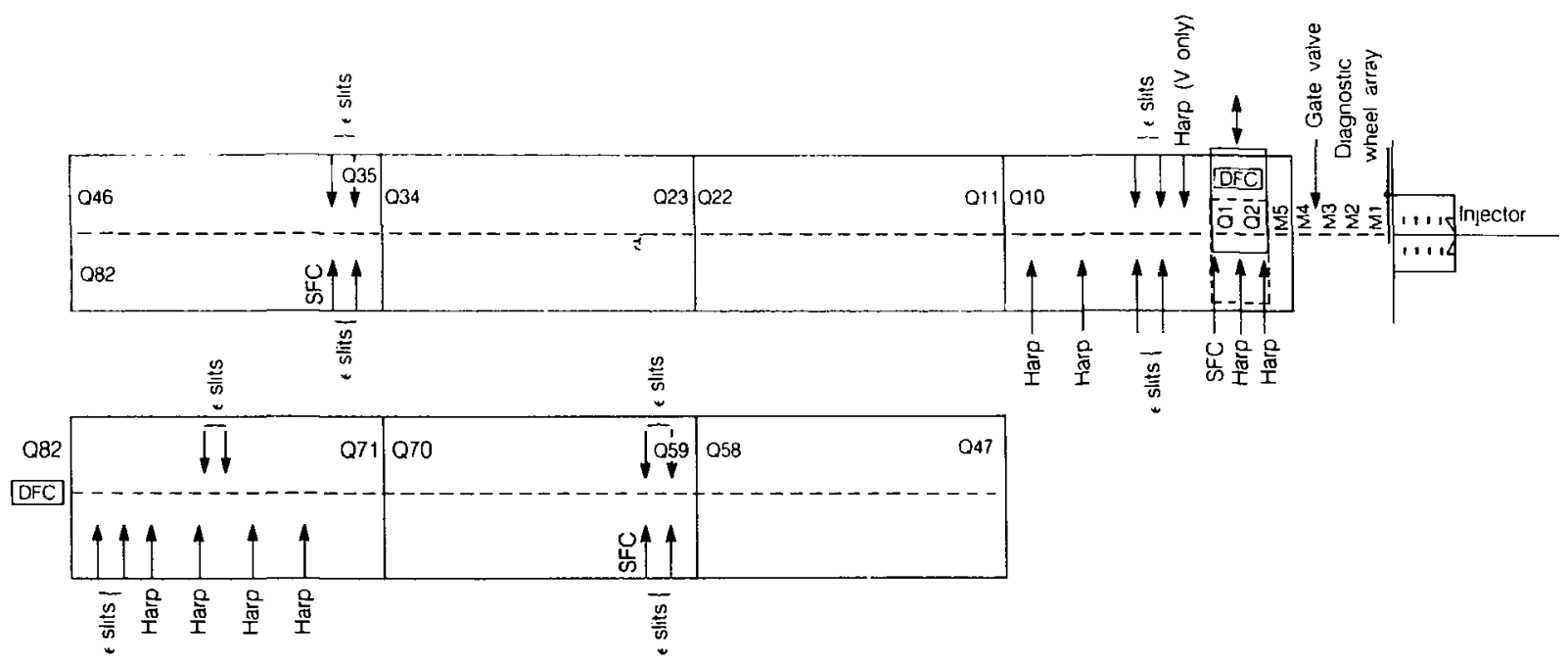

XEL 86410368

Figure 3.1: Physical layout of the SBTE lattice and diagnostics, showing the various charge collectors and
beam profile diagnostics along the channel. 


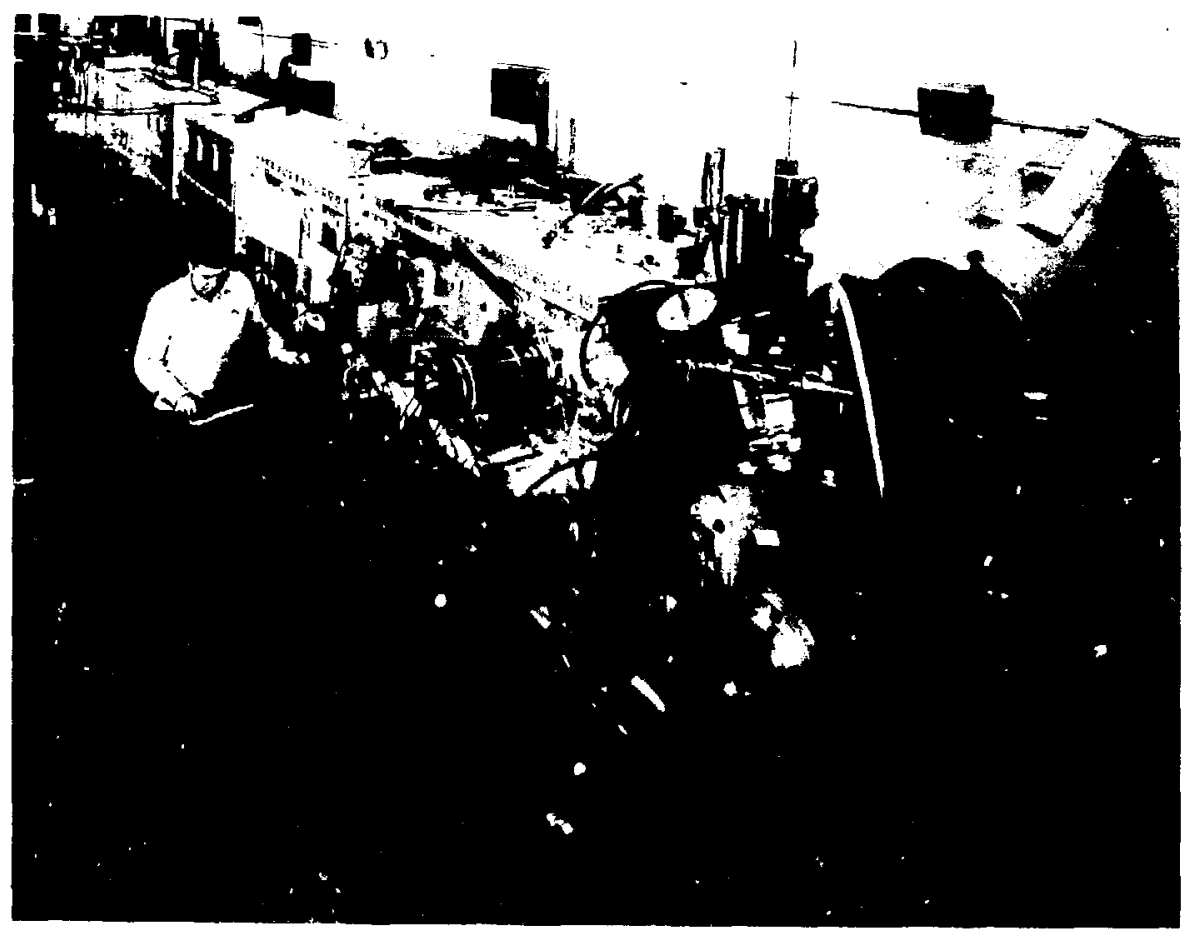

Figure 3.2: External view of the SBTE apparatus showing the vacuum chambers and diagnostic ports. Pumps are on the opposite side. 
procedure was not repeated after the tanks were installed on the beamline, for fear of distorting the quadrupole array (also aluminum). After adding two liquid nitrogen cold fingers to enhance water vapor pumping, the ultimate pressure was measured to be 8-10 $\times 10^{-8}$ Torr, with pressures of $2 \times 10^{-7}$ Torr being more common. Background gas effects on the beam are discussed in sections 6.3 and 7.9.

\subsection{Ion Source and Injector Assembly}

A cesium-loaded alumino-silicate ion source was chosen on the basis of literature reports, confirmed by our own preliminary investigations, that many milliamperes per square centimeter of $\mathrm{Cs}^{+}$can be extracted in short pulse, low duty factor operation [32,33]. The intrinsically low gas load, simplicity of operation, potential availability of certain other ion species with minimal effort, and the commercial availability of usable sources were the deciding factors. Such solid-state sources have two decided advantages over gaseous sources, in that the gas load for the solid-state source is nearly negligible, and there is a well-defined emitter surface, having effectively zero equilibration time before coming into steady-state operation. Very short pulses are possible ( $<10 \mu \mathrm{sec}$ ), precluding any space-charge buildup due to ionizing collisions between beam and background gas particles. Although the SBTE quadrupoles are electrostatic, and hence provide large sweeping fields to clear static charges from the bore region, this short-pulse feature could be desirable in magnetically focused intense beam dynamics research. More information about these sources, the problems we encountered, and our solutions is provided in Appendix $\mathrm{E}$.

A schematic diagram of the injector assembly (designed by C. Kim using Herrmannsfeldt's EGUN program [34]) is shown in Fig. 3.3, and an exploded view of the injector in Fig. 3.4. At the gun exit, up to three grids were 


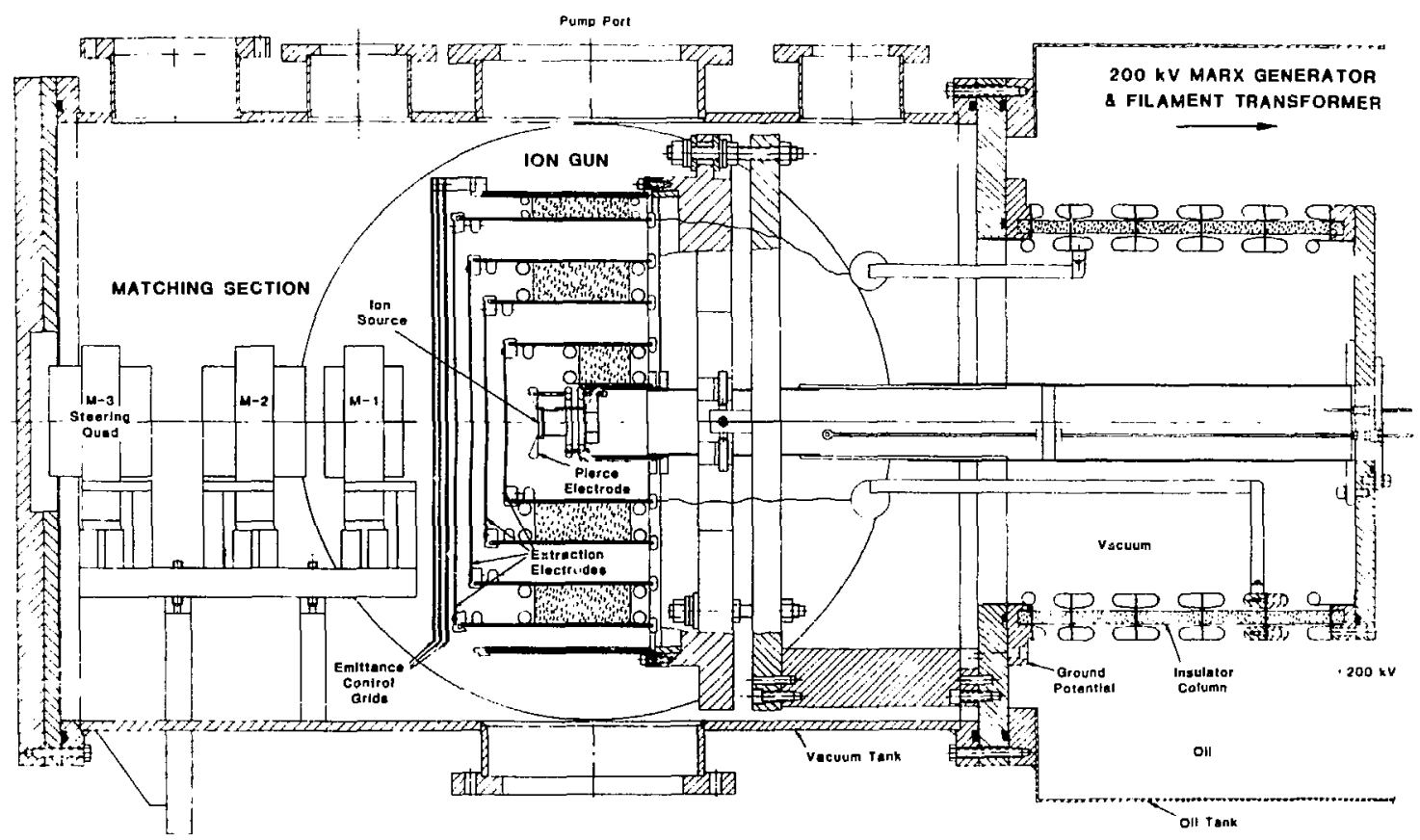

SINGLE BEAM TRANSPORT EXPERIMENT GUN A MATCHING SECTION

Figure 3.3: Schematic drawing of the SBTE injector, showing the concentric electrodes supporting the source and the focusing apertures. 


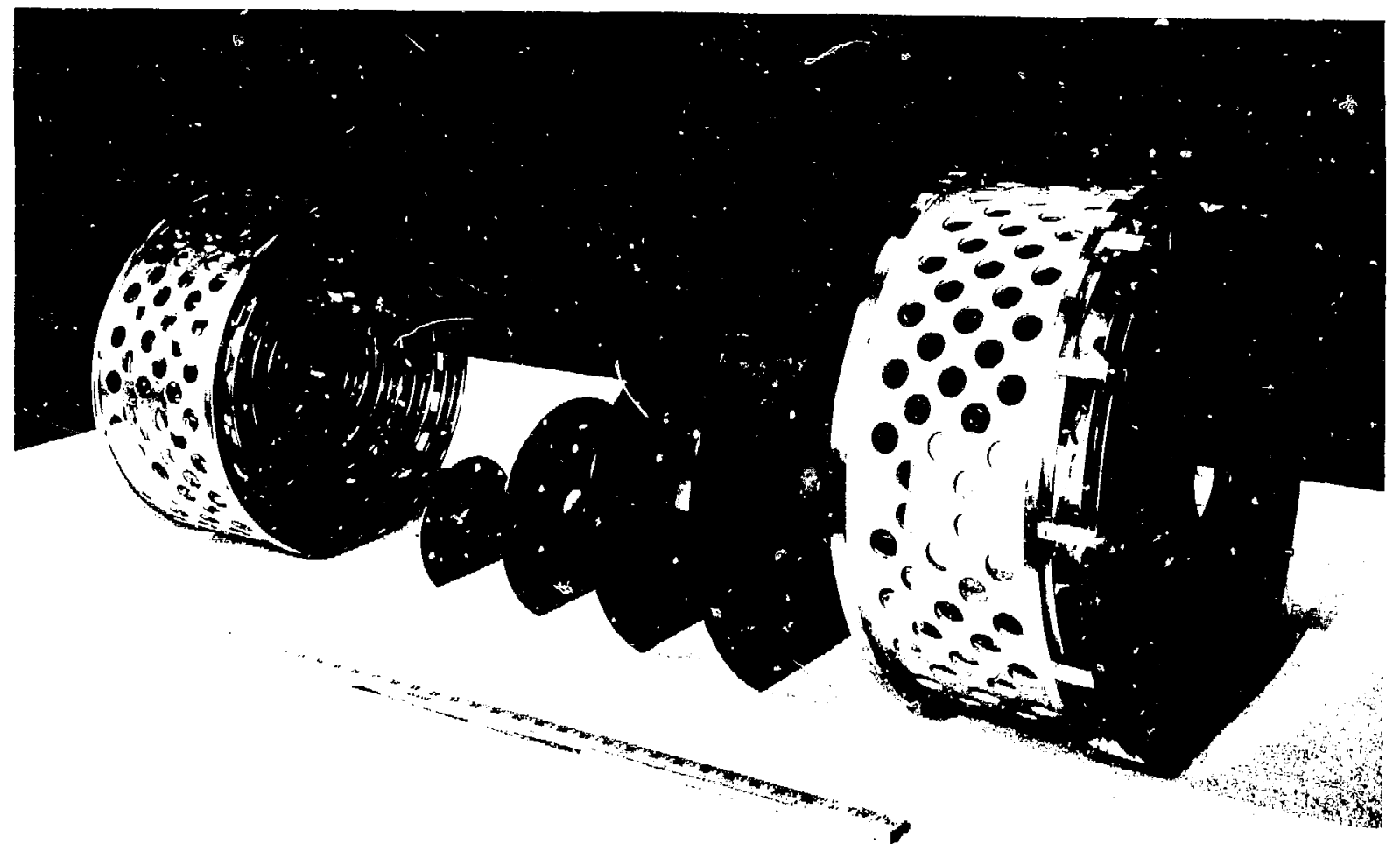

Figure 3.4: Exploded view of the SBTE injector, with the source visible at the center. 
provided, independently biasable for increasing the beam emittance prior ito matching into the periodic channel. Grid perturbations to the emittance are discussed in Appendix C. They were used to change the bea. emittance-tocurrent ratio continuously for the stability measurements reported in $\mathrm{Ch} .5$.

\subsection{Marx generator}

The high-voltage pulses $(120-160 \mathrm{kV})$ used to generate the ion beam were provided by a Marx generator, schematically represented in Fig. 3.5. It consists of twelve capacitors connected in parallel by a network of chargeliruiting resistors, with fast series connections made possible by triggering the breakdown of an array of spark gaps.

The high voltage pulse from the generator has a risetime of about $400 \mathrm{~ns}$, providing a good approximation to the ideal applied voltage pulse required to minimize current and beam energy transients during the initiation of the diode [35]. The output voltage droops in time as the charge on the capacitors flows out along the charging resistors and through the spark gaps and load resistance. We used a capacitive divider to obtain the intermediate voltages we needed to grade the ion gun apertures. Any droop in beam energy resulte in debunching of the entire beam pulse, making corrections necessary to the measured current to detect beam loss along the lattice as explained in section 4.2 .

\subsection{Quadrupoles \& Power Supplies}

We chose to use electrostatic quadrupoles, as mentioned above, primarily for cost reasons, but there are advantages in terms of the focusing force available from an electrostatic system. The beam current in the space-charge dominated regime scales as $(R / L)^{2}$ for constant $\sigma_{0}$, and the required foc using gradient scales as $1 / L$ for constant $\sigma_{0}$, where $R$ is the bore radius and $2 L$ 


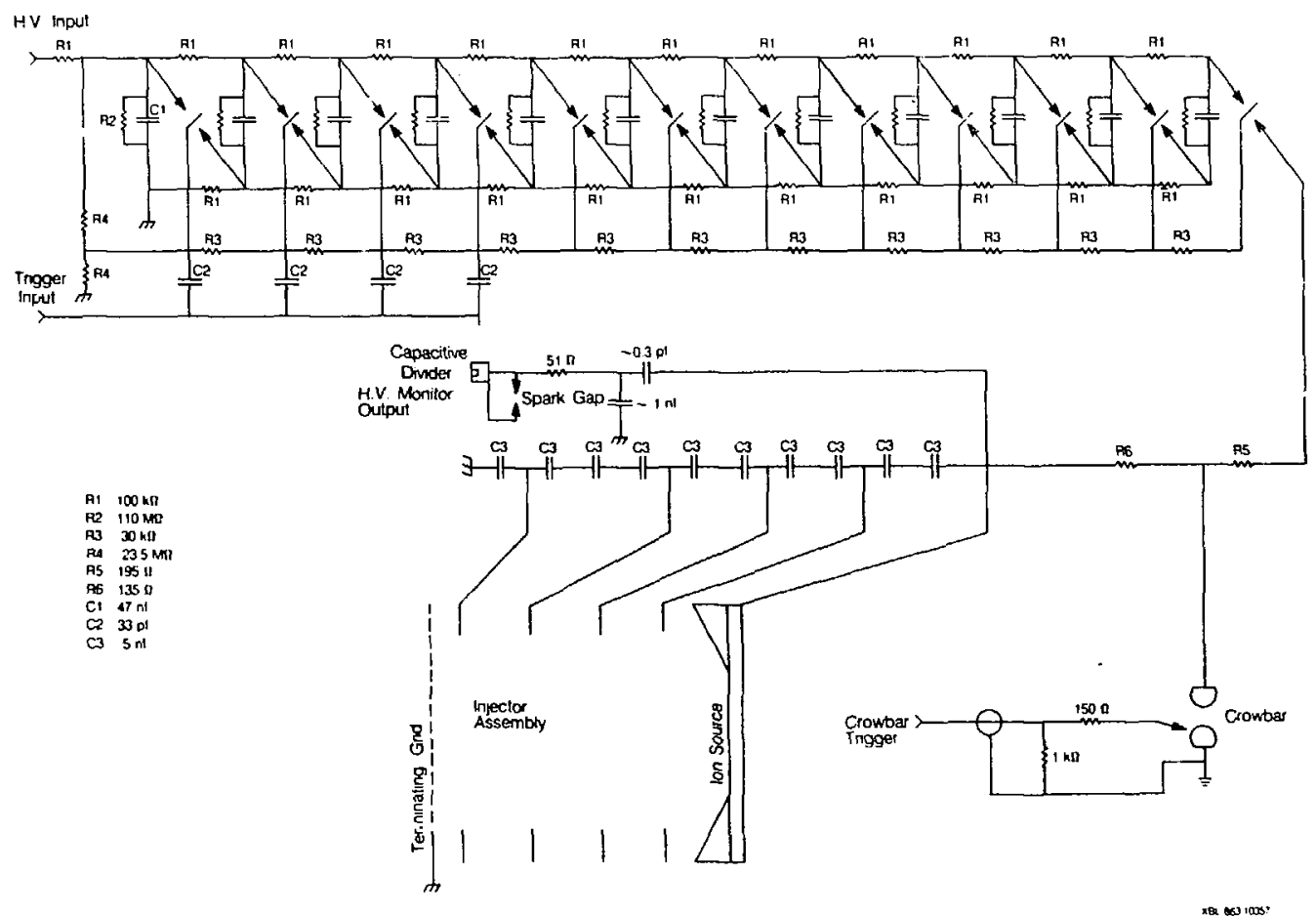

Figure 3.5: Circuit diagram for the SBTE Marx generator, showing the divider circuitry for the high voltage monitor and for the injector aperture voltages, as well as the triggers for the Marx itself and for the crowbar used to terminate the beam pulse. 
is the lens period. We needed a short lattice period in order to fit as many periods as possible into the available experimental area and to keep the beam current high, making beam diagnosis easier. These requirements made large focusing forces desirable. At the low particle velocity we planned to use $\{v \simeq 0.0014 c)$, the magnetic equivalent for the final geometry we chose would have required either pole-tip fields up to about $4 \mathrm{~T}$ or a much lower particle energy and beam current. The equivalent electric field of about $20 \mathrm{kV} / \mathrm{cm}$ was easily attainable.

The aim of the experiment was to measure the threshold for collective emittance degration in the most ideal linear field case possible. Short period and large bore requirements conflict with the desire to optimize focusing linearity. The bore and period of the lattice were chosen as a compromise between the desire to have a large enough beam size and curient for ease of diagnosis, and the need to keep the period short as mentioned above. Maschke also made an experiment on beam transport, using a very small lattice bore [36], but by doing so he essentialiy tliminated the possibility of making detailed phase space measurements on the beam.

Dr. L. J. Laslett calculated a pole-tip shape to optimize the focusing linearity of the lattice, providing a focusing field approximately sinusoidal in $z$. The resulting electrodes have a length of 4 inches, a quadrupole bore diameter of 2 inches, and an inter-lens gap of 2 inches. The overall period of the lattice is therefore 12 inches. More details are given in Appendix D, including a diagram of the shape of the electrodes.

In order to use the acceptance of the channel efficiently, it was desirable to "match" the beam into the transport channel, that is, to launch the beam so that its envelope has the same periodicity as the channel. We used the first five quadrupoles for this purpose. For this matching section (labeled M1- 
M5), the tore and sfacing of certain quadrupoles are different from those in the periodic lattice, in order to ease the matching of very high space-charge beams into the periodic lattice. We found that M3-M5 could have the same dimensions as the periolic lattice quadrupo'es, but M1 must be shorter in length ( 3 inches), M2 must, be larger in bore ( 2.5 inches in diameter), and the spacing between $\mathrm{M} 1$ and $\mathrm{M} 2$ must be as small as practical ( 1.25 inches). In order to counter the space-charge defocusing, some large quadrupole strength is needed from the ou'set. By making M1 short and close to M2, the expansion of the beam dimension defocused in M1 is minimized. Providing a wide bore for $M 2$ further relaxes the constraint on the allowable strength of $\mathrm{M1}$, allowing the M1-M2 doublet enough strength to focus a high-current beam from the injector, even with an initially divergent envelope. The beam size in M3 is also calculated to become large in extreme cases, but the M2 constraint is calculated to be more severe. We also provided a separation between M3 and M4 of 2.5 inches to allow room for a gate valve, rather than using the periodic lattice spacing of 2.0 inches. This valve allowed access to the source housing while leaving the transport section under vacuum, greatly reducing the outgassing time required to return to high vacuum after working within the source chamber.

Four quadrupoles would be enough in principle to match the beam radius and convergence in each transverse dimension from the source into the periodic lattice. However, the use of a fifth independent matching element allowed additional control over the aperture requirements during matching. This freedom was useful later, when the beam began showing noticeable aberrations in focusing at the exit of the matching section as we tried to match the beam into lattices with $\sigma_{0} \sim 90^{\circ}$. This effect was reduced by weakening the M1-M2 doublet (see section 5.1.1), and may have been directly due to the matching section fields. 
The transport quadrupoles were bench-mounted on I-beams, each beam holding 12 quadrupoles (see Fig. 3.6). The centers of the lenses were held at their nominal positions to within \pm 0.003 inch, and the relative lens rotation about the beam axis was held to \pm 3 milliradian. Each group of 12 quadrupoles was aligned within the vacuum tanks to within 0.010 inch using an optical surveying transit with precision targets.

All quadrupole power supplies are unregulated $30-k V$ supplies. They are used in \pm pairs to maintain a true ground potential along the beam axis, apart from the beam potential. The connection to the quadrupoles is made through a filter and monitor network shown in Fig. 3.7.

\subsection{Diagnostics}

Diagnostic equipment consisted of various beam collimators, charge collectors, and also a multiwire scanner ("harp") used in the secondary electron emission mode. Two types of free-standing Faraday cups were used-shallow Faraday cups (SFC's), with grids for secondary electron control, and deep Faraday cups (DFC's), which were gridless, but electrostatically insulated for electron control.

\subsubsection{Multiwire Profile Monitor}

The multiwire profile scanner ("harp") is an array of thirty wires 2 inches in length and 0.010 inches in diameter, with a spacing between centers of 0.050 inches. The wires are individually connected to a $1000-\Omega$ termination across the input terminals of a follow-and-hold circuit, as shown in Fig. 3.8. The various signal levels are frozen in when the follow-and-hold array is triggered, and then multiplexed on a $50-\Omega$ coaxial cable to a digitizing storage ascilloscope. A minicomputer then reads the signal levels from the digitizer for calculation of the beam centroid and RMS radius. For one small subset of the data, we also examined the third moment of the transverse distribution 


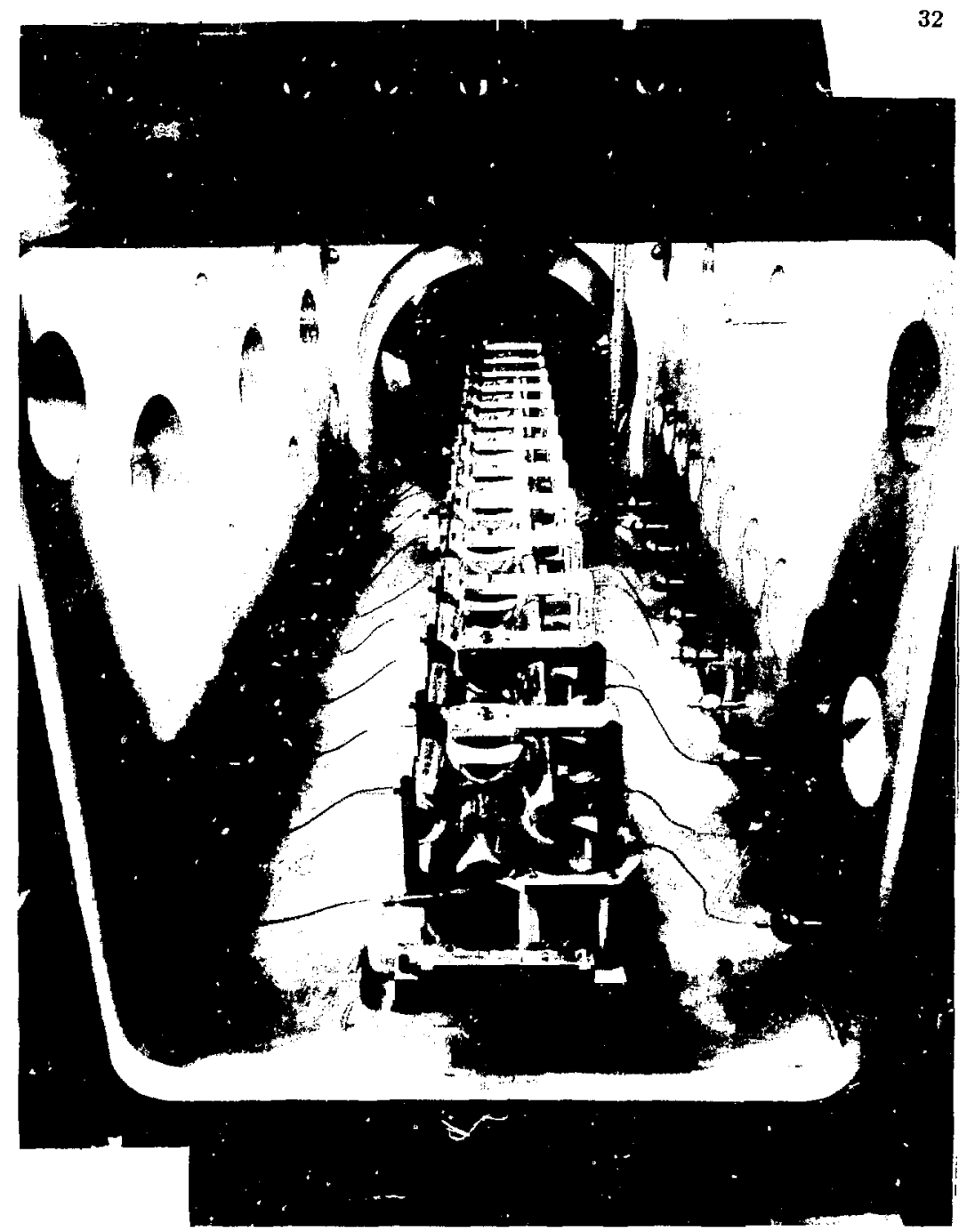

Figure 3.6: Interior of one of the SBTE vacuum tanks with quadrupole array installed. The length of each quadrupole electrode is $\mathbf{4}$ inches, and the bore radius is 1 inch. 


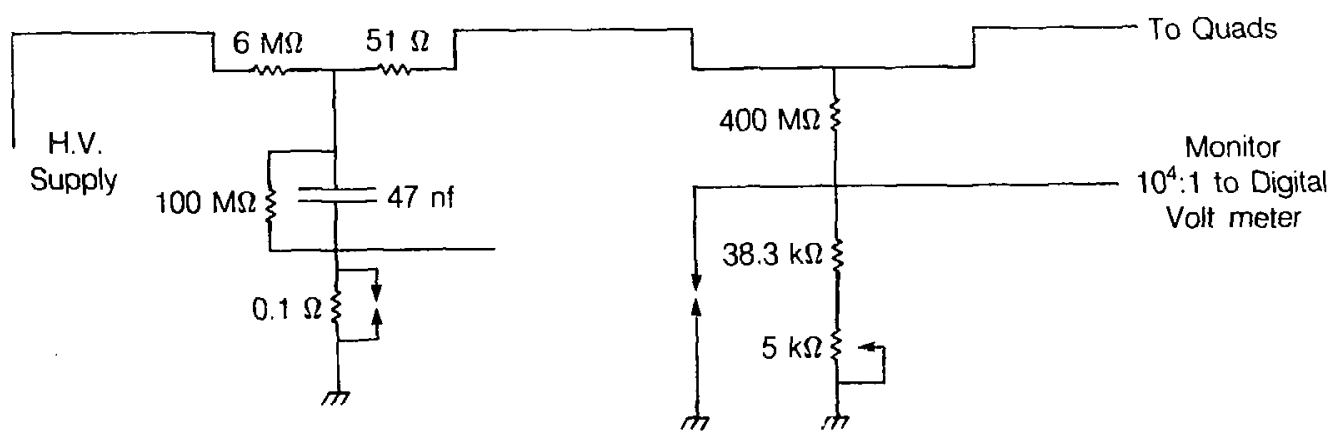

Quadrupole Power Supply Stifferier and Monitor Circuitry

XBL 863.10353

Figure 3.7: Circuit diagram for the SBTE quadrupole power supply and monitor chain. The 47-nf capacitor serves to hold the quadrupole potential constant in case of beam-induced currents. The monitor circuit measures the voltage applied to the quadrupoles. 


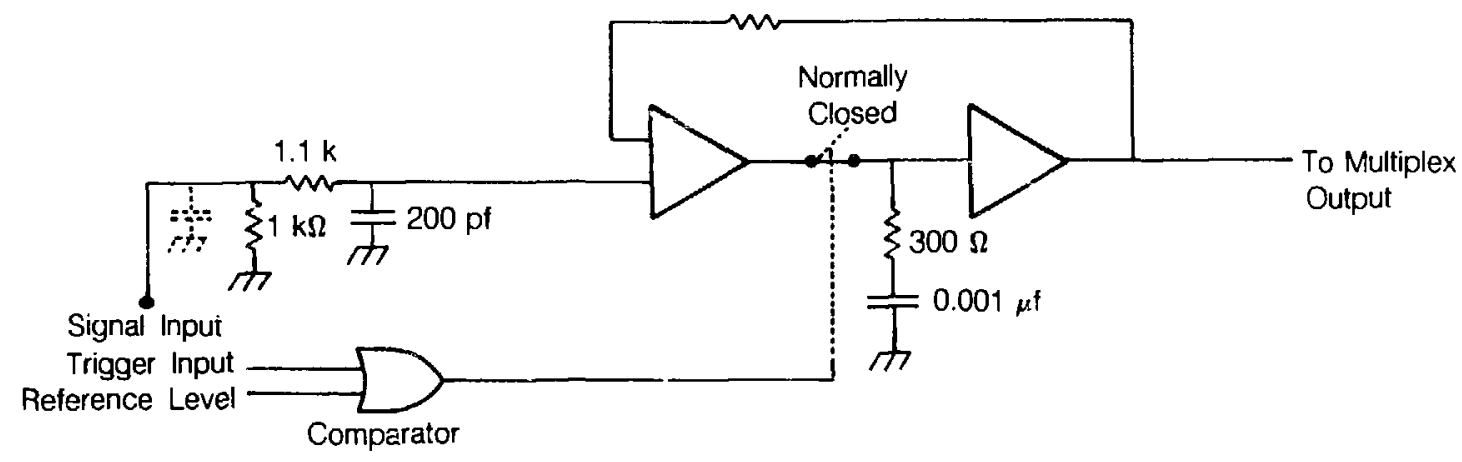

XBL 863.10354

Figure 3.8: One of an array of thirty follow-and-hold circuits for the harp profile monitor. When triggt:red, the comparator opens the transistor swich between the op-amps, freezing the voltage on the 1-nf capacitor. 
for comparison with analytical results for a sextupole-symmetry beam mode with a non-zero third moment (see Figs. 5.5 and 5.4). We also used the emittance diagnostics to measure beam profiles.

\subsubsection{Beam current monitors}

The SFC's (Fig. 3.9) must be thin enough for insertion into the 2-inch gap between quadrupoles, which have electrodes at $\pm 15-\mathrm{kV}$ potentials, without shorting out the quadrupoles. Grids were required to shield the collector from the quadrupole fields and to provide a bias field to retain the secondary elections on the main collector. These grids have an overall transparency of between $90 \%$ and $96 \%$. The high yield of secondary electrons (measured to be about 11 per ion impact for $120-160 \mathrm{keV}$ cesium ions normally incident on stainless steel) could have had a $50 \%$ effect on the measured beam current. The gridless DFC's (shown in Fig. 3.10) provided a current measurement with errors due primarily to secondary positive ion emission. These DFC's were designed by Kim, using the EGUN program [34], to provide secondary electron control by electrostatic effects, without inserting grids into the beam.

We placed gridded shallow Faraday cups, varying in grid transparency and mechanical design, along the lattice after Q2, Q6, Q36, and Q60. The two identical deep Faraday cups were placed after M5 and at the end of the lattice after Q82. We could install one of the DFCs within the lattice only by providing for a precision transverse-traveling mount holding the DFC itself and the two quadrupoles displaced by the DFC. We mounted Q1, Q2, and the DFC on a metal plate and aligned the plate carefully within the quadrupole lattice. Transverse motion was provided by a ball screw in the same manner as for the other diagnostics. These two cups provided direct confirmation of constancy of the beam current through the lattice observed at the lower $\sigma_{0}$ values. This constant beam current was used to intercal- 


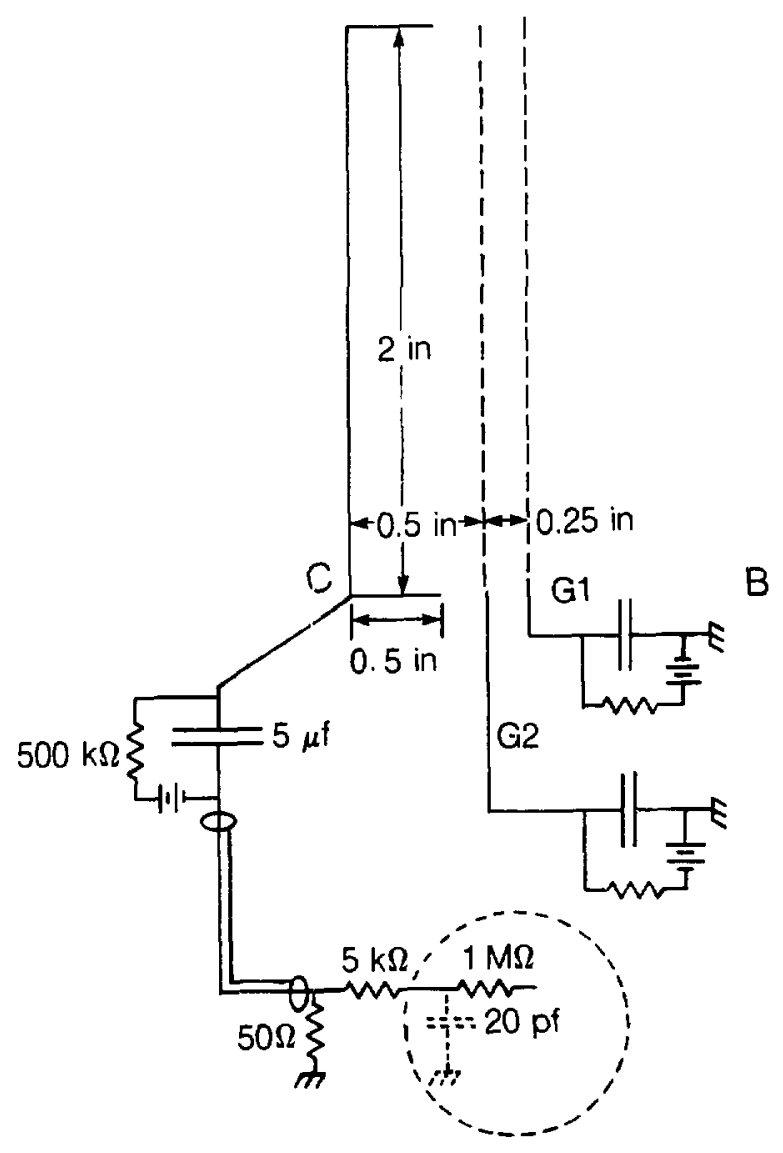

Shallow Faraday Cup

XBL 864-1036\%

Figure 3.9: Diagram of the shallow Faraday cup, including bias and signal-measurement circuitry. The 5-k $\Omega$ resistor in series with the oscilloscope (represented by the dotted circle) served as a $100-n$ filter. 
37

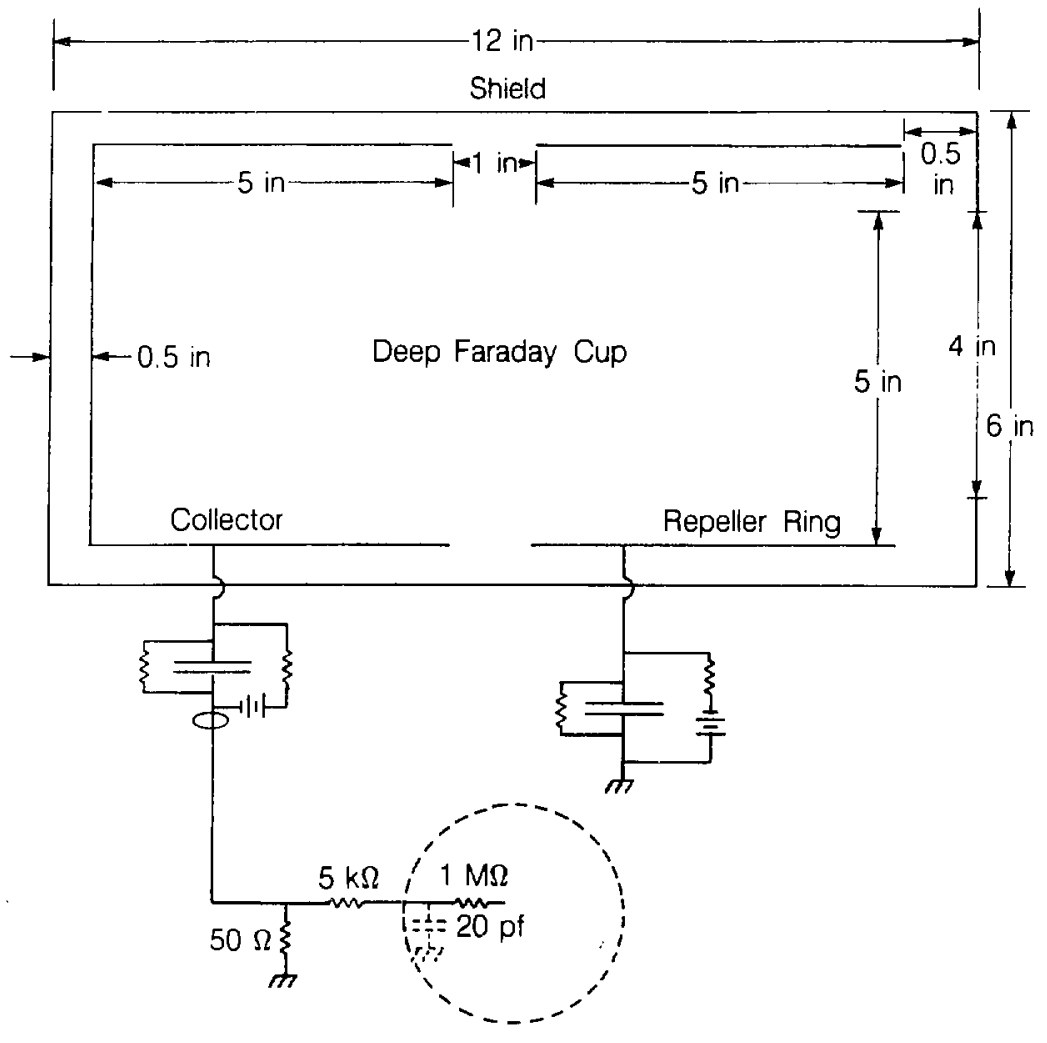

Deep Faraday Cup with Bias and Detector Circuitry

XBL $863 \cdot 10355$

Figure 3.10: Diagram of the deep Faraday cup. Gridless design required deep electrodes to provide bias for electron control. The typical operating potentials were $\pm 2 \mathrm{kV}$ on the collector and repeller, respectively. 
ibrate the SFC's, which differed in current calibration becuase of differing grid transparency (see Fig. 5.3). No cup, however, showed detectable variation in its current response with time, and all cup measurements were made using a single 49.9- $\Omega$ Allen Bradley carbon composition resistor. (Metaİ-film resistors were too sensitive to damage caused by bias breakdown and other high-voltage transients.) Details of cup bias behavior and calibration are given in Appendix B.

\subsubsection{Phase space diagnostics}

Emittance measurements were made with pairs of 0.010 -inch slits, placed in successive midplanes between quadrupoles (ground planes in the absence of beam). The principle is shown in Fig. 3.11. The presence of the grounded slits did not disturb the quadrupole fields, and in fact enforced the ground plane boundary condition when the intermediate quadrupole was grounded to provide a drift region between the slits. Rather than require a second drift to one of the SFC's, we incorporated a miniature slit cup with the second slit, as shown in Fig. 3.12 .

In the emittance measurements, where we were not concerned with absolute current, we used a negative bias on the cup collectors, taking advantage of the secondary electron amplification. Operational characteristics and bias response are discussed further in Appendix B.

\subsubsection{Diagnostic positioning}

The diagnostics were positioned within the vacuum system along ball screws, with the driving torque applied from the outside using commercially available ferrifluidic seals, having a very low gas leakage. The positioning precision within the beamline was within \pm 0.002 inch 


\section{Principle of Two-slit \\ Emittance Measurement Ter.hnique}

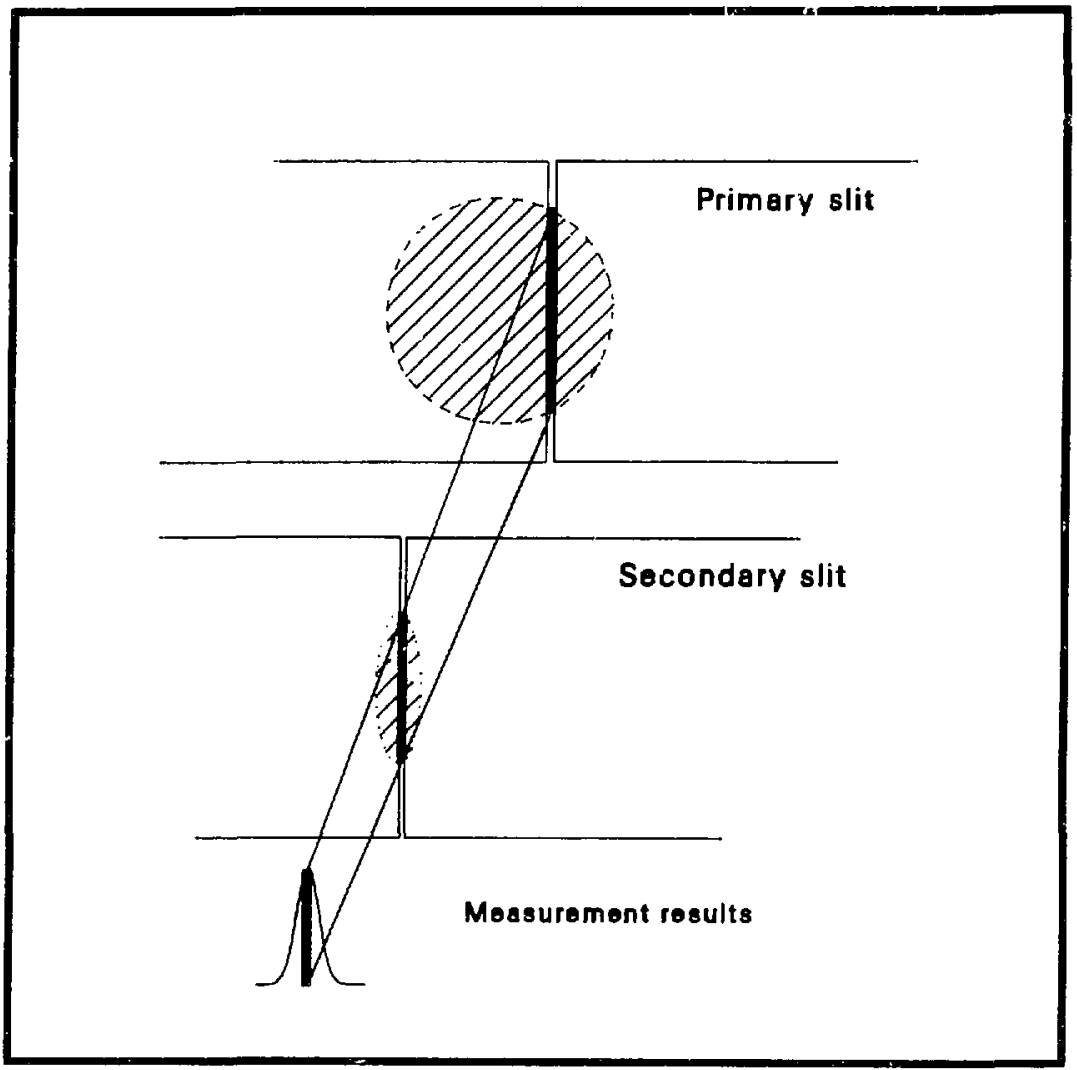

XBL $865-1843$

Figure 3.11: Illustration of the principle of the two-slit phase space measurement technique. The particles are collimated in position by the upstream slit, and the downstream slit passes those particles into the detector which have a certain well-defined transverse velocity. 


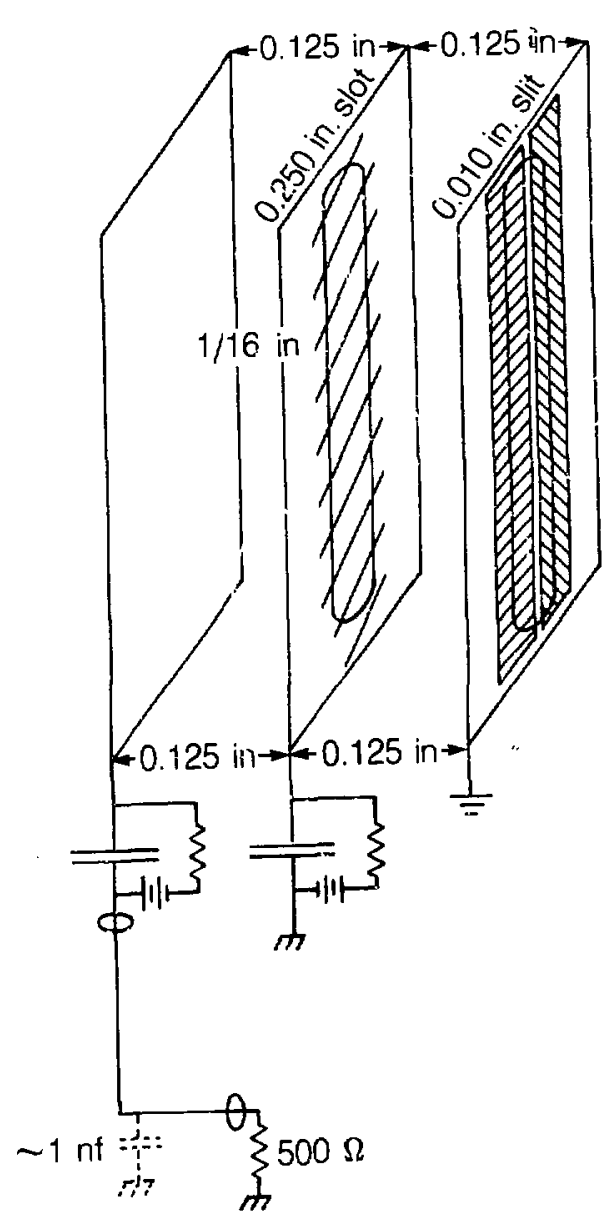

40

Slit Collector Cup

Figure 3.12: Diagram of the detectors we used for the slit measurements. The wires across the intermediate electrode provided a well-defined electrical surface over the slot. 


\subsubsection{Attenuator Wheel}

We provided 3 wheel array (see Fig. 3.13) of attenuators and small apertures just cownstream of the source and emittance grids. The attenuators were mesh screens 1.5 inches in dianneter, ranging in transparency from $3.5 \%$ to $95 \%$. The beam had a diameier of about 1 inch at the wheel location, and was well-centered within the apertures to avoid clipping part of the beam. We also provided several small apertures for current density scans across the source. We also used them for making small beamlets at various $(x, y)$ positions over the source area to check source alignment and single-particle lattice properties. The small apertures vere of $2 \mathrm{~mm}$ and $3 \mathrm{~mm}$ diameter; the $3 \mathrm{~mm}$ apertures were covered by a $50 \%$ transmission mesh. The motion across the source was nearly horizontal, because of the 4.75-inch radius from the center of the wheel to the center of the beam. Discrete vertical resolution was made possible by using apertures offset along radial lines from the wheel center, but was much poorer than the horizontal resolution.

\subsection{Data Acquisition System}

Data acquisition for phase space measurements and harp readout was done through a Tektronix 7D20 digitizing storage insert. in a Tektronix 7904 oscilloscope, coupled to an EP-85 computer through the HPIB (IEEE-488) interface. The overall trigger and communication loop is shown in Fig. 3.14. The HP-85 controis the slits via a stepper motor interface, triggers the Marx generator and oscilloscope, and then interrogates the oscilloscope for the signal at a manually preset time. The HP -85 checks that the signal recorded by the oscilloscope is within the amplifier range setting, resets the amplifier gain, moves the slit(s) unless the signal was out-of-range, and continues the loop. The amplifier gain was set to keep the signal level near $1 / 3$ of full-scale. This value was chosen because as the slits entered the beam, the beam signal 


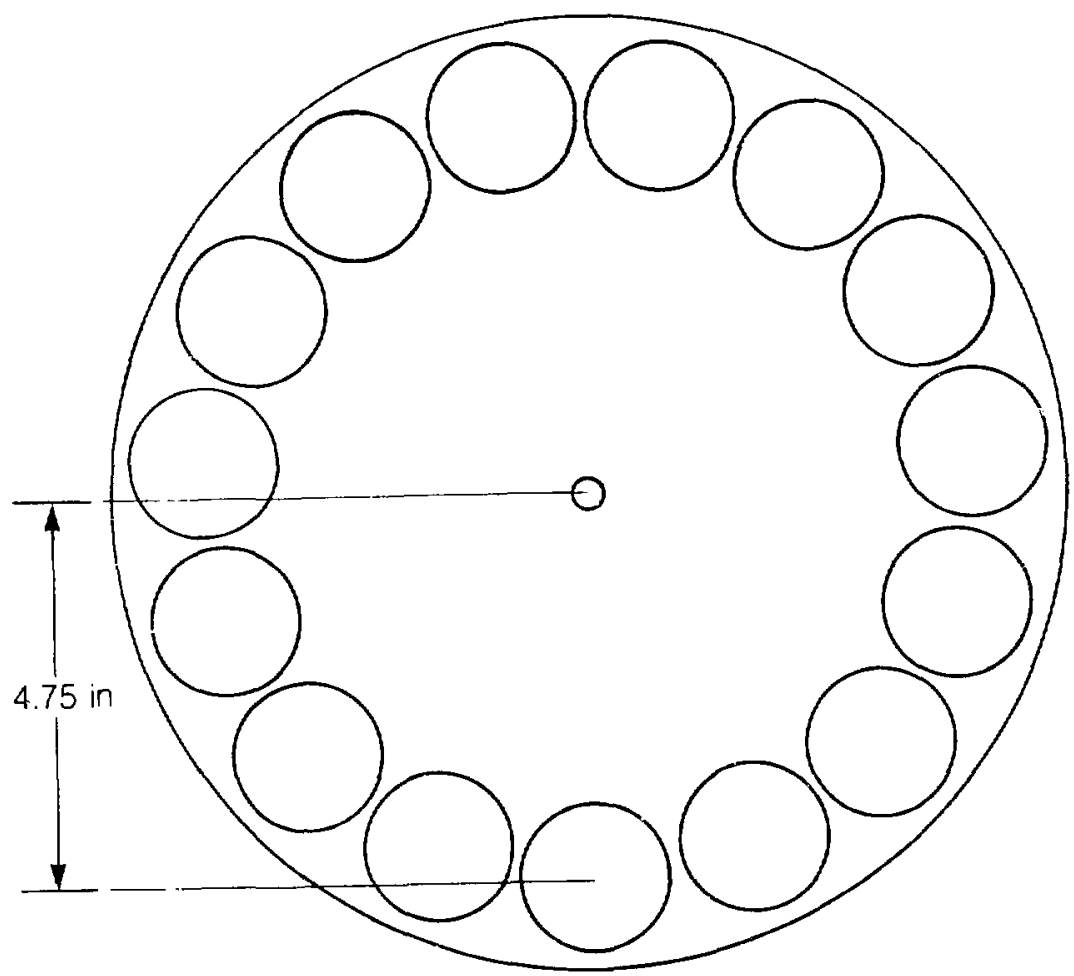

XBL 863.10352

Figure 3.13: Diagnostic wheel assembly. The holes in he wheel were of 1.5-inch diameter to avoid clipping the beam edges. Various attenuator grids were provided, along with blank inserts having either $2-\mathrm{mm}$ or 3 -mm diaineter holes to generate beamlets for diagnostic purposes. 


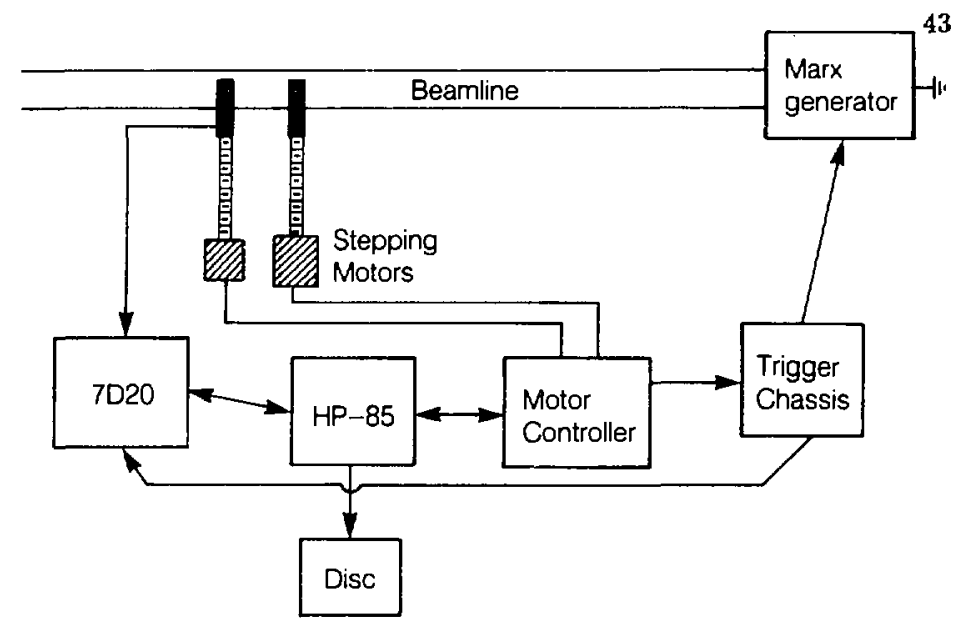

Automated data acquisition network physical layout

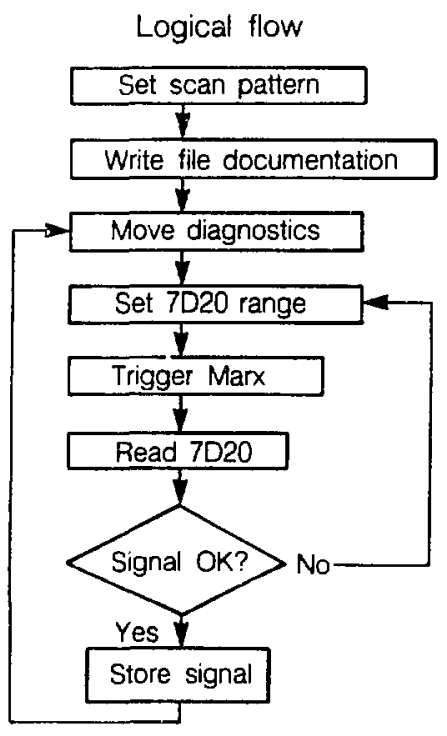

XBL 863.10350

Figure 3.14: HP-85 based data acquisition system layout and flow chart 
rarely rose more than a factor of 3 from point to point. This minimized the need for repeating data measurements during the scans, while providing good resolution in the tails of the distribution. 


\section{Chapter 4: Lattice Performance and Data Analysis}

In this chapter we present details of the SBTE beam and diagnostics and of the analytical techniques used to interpret the data. We discuss diagnostic performance and calibration, particie energy calibration, background gas interaction with the beam, and how actual performance compared with the design parameters for the source. Errors are discussed in Ch. 7 .

The SBTE was assembied in stages, with procedures and diagnostics changing from time to time as a result of measurements and growing experience. The source was installed first, and during its checkout we determined what size slit we should use for emittance measurements. As the lattice was gradually installed, ve tested our Faraday cups, emittance measurement procedures, and beam tuning procedures, inproving each in the process. We found that we needed Faraday cups having no grids to intercept beam if we were to get absolute measurements of beam current, and that we needed to provide automated data collection and storage to improve the resolution of the phase space measurements and facilitate data analysis. When we found that we had not reached the low-emittance stability limit of current-dominated beam transport for $\sigma_{0}<90^{\circ}$ with our initial bearn parameters, we shortened the injector assembly to double its current output. Finally, we incorporated charge collectors with the downstream slitis of our emittance measurement apparatus to increase the area in phase space arcessible to measurement (the diagnostic acceptance). Although some data were taken during this process of change, almost ill of the data reported below were taken after these changes were compleied.

\subsection{Injector Perveance and Performance}

The "perveance" of a beam from a diode is the ratio $I / V^{\frac{3}{2}}$, where $I$ is the current of the beam and $V$ is the energy of the particles in eV. It is a 


\begin{tabular}{|c|c|}
\multicolumn{1}{c}{ Device } & Generalized perveance \\
\hline SBTE & $5.5 \times 10^{-3}$ \\
\hline proposed HTE & $<2 \times 10^{-6}$ \\
\hline proposed driver & $1-5 \times 10^{-4}$ \\
\hline
\end{tabular}

Table 4.1: Generalized perveances for SBTE and for two proposed accelerators

measure of the intensity of the space-charge forces in the beam. A related quantity, defined for a beam without regard to its source, is the dimensionless perveance found in the envelope equations of section 2.2 .2 , called the generalized perveance, $Q$. It is defined in the nonrelativistic limit by

$$
Q=\frac{q I}{\pi \epsilon_{0} m v^{3}} \quad\left(=\frac{1}{4 \pi \epsilon_{0}} \sqrt{\frac{2 m}{q}} \times \frac{I}{V^{\frac{3}{2}}}\right)
$$

The space-charge forces of the SBTE beam are large compared to those of benms proposed for HIF use, as we show in Table 4.1. The SBTE beam is by this measure an order of magnitude more intense than the beam required to drive a HIF target. Even at the low-energy end of the HIF driver, where space-charge effects are most severe, the generalized perveance of each beam of the driver in some scenarios is lower than that of the SBTE beam. In a driver for HIF, the anticipated total number of ions is equivalent to about $300 \mu \mathrm{C}$ of "particle" charge, and the final energy at the target will be about $10 \mathrm{GeV}$. For a 5- $\mu$ sec-long pulse at the source, using 100 beams (for the initial period of acceleration, merging them into about $10-20$ beams as the energy increases) [37), the current per beam is about $0.6 \mathrm{~A}$. If the snurce energy can be made as high as $3 \mathrm{MV}$ (it must be as high as technologically possible, because in low-velocity, low-current operation, an induction linac is very inefficient) then we have, for singly charged ions, that $Q \sim 2 \times 10^{-3}$. 
We compare the values of the beam radius and divergence calculated by Dr. C. Kim [38] with the measured values as a function of current from the injector in Fig. 4.1. The beam parameters are varied by changing the voltages on the various electrodes while maintaining the output voltage at a constant value. The potential drop between the source and the first aperture plate controls the source current, and the variation of the accelerating field along the injector determines the focusing applied to the beam, and hence the optics of the beam at the injector exit. The difference between calculated and measured beam quantities can easily be explained by thermal expansion of the aperture plates, and the consequent alteration of the electrode spacing and accelerating gradient from the design values. In a bench test, we applied a hot air gun to the final aperture plate (the largest and most sensitive to thermal expansion effects) and we observed a 0.2 -inch deflection of the final aperture to result from an approximately $50^{\circ} \mathrm{C}$ temperature change. This degree of motion of the plate would alter the final optics somewhat.

\subsection{Particle Energy and Lattice Strength Calibration}

The monitor cliains for the quadrupole power supplies shown in Fig. 3.7 were well-calibrated. The error in measurement of the beam energy is the major source of uncertainty in the $\sigma_{0}$ calibration of the lattice. The Marx generator output, with an RC decay time of about $0.6 \mathrm{msec}(0.15 \%$ per $\mu \mathrm{sec}$ droop) is coupled to the various gun electrodes through a capacitive divider. We used pulses with durations of about $10 \mu \mathrm{sec}$. The drocp with time of the particle energy, and hence velocity, results in a slight debunching of the bearu as it fasses through the channel. The result for linear debunching is

$$
\frac{1}{I} \frac{\partial I}{\partial z}=\frac{1}{2 v_{z}} \frac{1}{V} \frac{\partial V}{\partial t}
$$



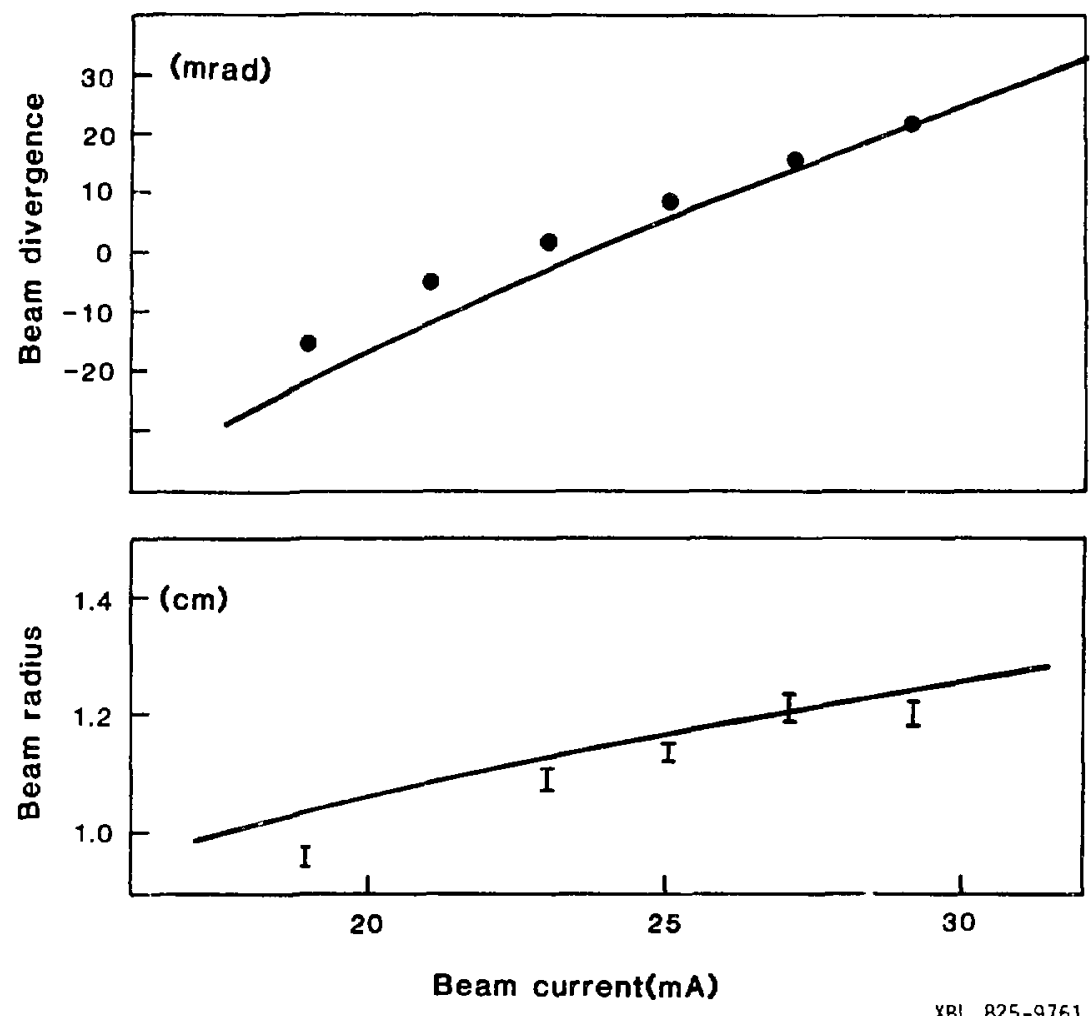

Figure 4.1: Comparison of measured and calculated injector output, with the initial injector design, operating at $160 \mathrm{kV}$. The agreement with the design values is good. 
where $I$ is the beam current, $V$ is the beam energy, $z$ is distance along the channel, and $v_{s}$ is the velocity of the beam along the channel. The calculated current droop for $120 \mathrm{keV}$ particle energy is only about $2.5 \%$ over the length of the channel ( $\sim 13$ meters). This energy variation also causes the beam centroid to move transversely with time within the channel. This is due to misalignments and the variation in focusing properties of the lenses with particle energy. This sweeping phenomenon from head to tail of the bunch was most noticeable at high lattice strengths, for which the misalignment effects give larger beam offsets.

We expect the calculation of the $\sigma_{0}$ values of the lattice as a function of particle energy and applied field to be very accurate. The major error is due to inexact measurement of the focusing field and particle energy. The calibration of the gun voltage was made with a capacitive divider, directly monitoring the source voltage through the Marx tank oil dielectric. A schematic diagram is included in the Marx schematic in Fig. 3.5. The divider ratio was measured at low-voltage to be 3182:1. The only components subject to high voltage stress were the metal and oil elements in the Marx tank. These were coupled to the $50 \Omega$ cable which served as the large capacitor to ground in parallel with the oscilloscope. We know of no dielectric nonlinearity effects that could change the division ratio at the field strength in use.

All results presented here are based on the capacitive divider energy measurement, supported by results from a $90^{\circ}$ electrostatic energy analyzer [39] and by time-of-tlight measurements. The measurements reported here were made using $17.85 \pm .03 \mathrm{kV}$ as the charging potential on the Marx. The various energy determinations have errors of about $\pm 1 \mathrm{kV}$. We obtained $122.5 \mathrm{keV}$ from the divider, $123.0 \mathrm{keV}$ from the energy analyzer, and $122.0 \mathrm{keV}$ from the time-of-flight measurement. 
We measured $\sigma_{0}$ by displacing a low-current beam ar:d measuring the wavelength of the coherent oscillation as a function of the quadrupole voltage over the range $40^{\circ}<\sigma_{0}<80^{\circ}$. We used a beam current of $0.35 \mathrm{~mA}$, for which image-charge effects were calculated to be negligible. The results are shown in Fig. 4.2 and Table 4.2. We measured the beam centroid as a function of strength of the lenses Q6-Q82 at four points along the lattice, just downstream from Q5, Q35, Q59, and Q79. As the lattice strength is varied, the initial phase of a beam injected with nonzero displacement will shift. We injected the beam at zero displacement to elsure that the initial phase of the betatron oscillation was zero $\left(\psi_{0}=0\right.$, see Eqn. 2.3), giving the sine-like orbit for any lattice strength. The amplitude will grow smaller in a well-aligned lattice with increasing lattice strength, although the local offset in any one quadrupole may grow if misalignments cause the ideal orbit center to shift.

The results agree very well with calculations based on the measured beam energy over this range of $\sigma_{0}$, as shown in the table. We estimate that we have a $\pm 10^{\circ}$ range of error in the phase determination at each measurement point, giving us the value of the accumulated phase change $\Delta \psi$ over the 37 periods from Q5 to Q79 to within about $\pm 20^{\circ}$. This results in an uncertainty in the phase advance rate per period of about $\pm 0.6^{\circ}$. Values of $\sigma_{0}$ greater than $80^{\circ}$ were calculated from the applied lens voltage and measured particle energy.

\subsection{Beam Matching Procedures}

In order to calculate how to match the beam into the lattice, we need to know the initial $x$ and $y$ radius and divergence of the beam at the source. We could measure the horizontal properties much more accurately than the vertical properties. Because the beam did not depart from azimuthal symmetry enough to detect with our vertical rescution, we assumed this symmetry for 


\section{Centroid Position at $\mathrm{Q79H}$ vs. Quad Potential for Various $x$ ' Values, $0.35 \mathrm{~mA}$}

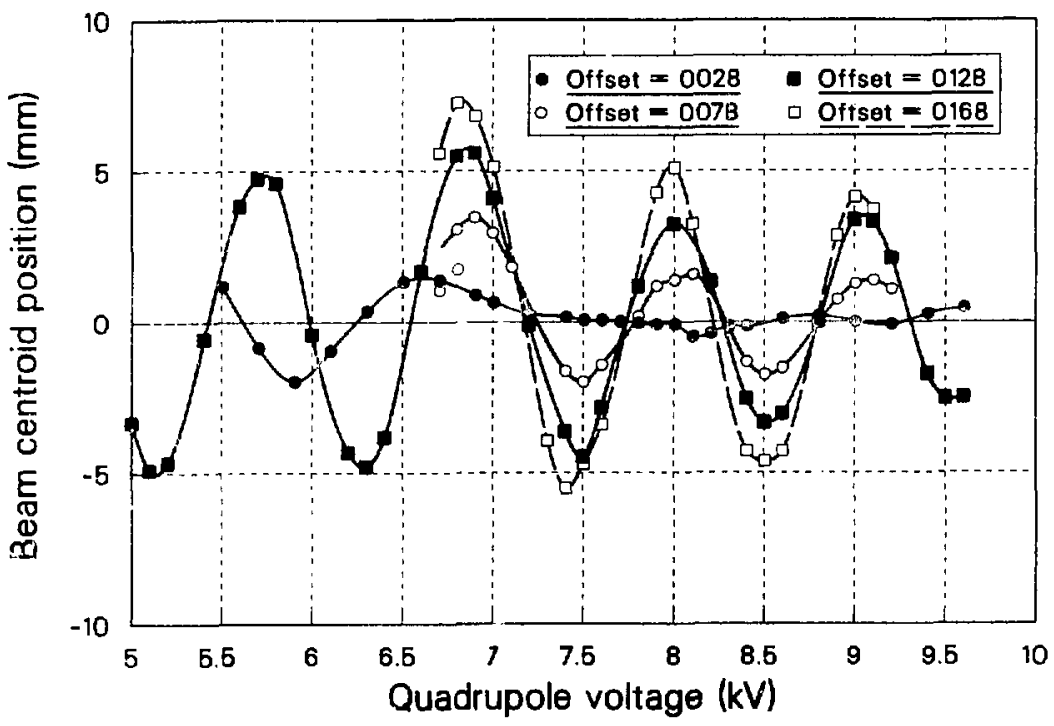

XCG $864-7156$

Figure 4.2: Direct measurement of $\sigma_{0}$ in the lattice. We provided a node in the beam offset at Q5, and measured the accumulated phase advance for the motion of the beam centroid in the lattice for low current, as a function of lattice strength. Dividing the total phase advance by the number of intervening lattice periods gives $\sigma_{0}$. The limits on the range of the quadrupole voltage for each curve were set by the onset of beam loss, which would alter the beam centroid artificially. The "offset" values in the legend do not refer directly to the offset of the beam. 


\begin{tabular}{|c|c|c|c|c|}
\hline Vo producing & no. of & measured $\sigma_{0}$ & \multicolumn{2}{|c|}{ calculated $\sigma_{0}$} \\
\cline { 4 - 5 } node at Q79 & oscillations & & $x_{\mathrm{p}} \simeq 0 \mathrm{~mm}$ & $x_{\mathrm{p}} \simeq 7 \mathrm{~mm}$ \\
\hline 5410 & 4.5 & $43.8^{\circ} \pm 0.6^{\circ}$ & $43.0^{\circ}$ & $43.2^{\circ}$ \\
\hline 5970 & 5.0 & $48.6^{\circ} \pm 0.6^{\circ}$ & $47.7^{\circ}$ & $48.0^{\circ}$ \\
\hline 6550 & 5.5 & $53.5^{\circ} \pm 0.6^{\circ}$ & $\mathbf{5 2 . 7 ^ { \circ }}$ & $52.9^{\circ}$ \\
\hline 7130 & 6.0 & $58.4^{\circ} \pm 0.6^{\circ}$ & $57.7^{\circ}$ & $58.0^{\circ}$ \\
\hline 7700 & 6.5 & $63.2^{\circ} \pm 0.6^{\circ}$ & $62.8^{\circ}$ & $63.1^{\circ}$ \\
\hline 8220 & 7.0 & $68.1^{\circ} \pm 0.6^{\circ}$ & $67.6^{\circ}$ & $67.9^{\circ}$ \\
\hline 8760 & 7.5 & $73.0^{\circ} \pm 0.6^{\circ}$ & $\mathbf{7 2 . 7 ^ { \circ }}$ & $73.0^{\circ}$ \\
\hline 9250 & 8.0 & $77.8^{\circ} \pm 0.6^{\circ}$ & $77.4^{\circ}$ & $77.8^{\circ}$ \\
\hline
\end{tabular}

Table 4.2: Comparison of values of $\sigma_{0}$ calculated from quadrupole voltage and particle energy with those from centroid measurements, using $122.5 \mathrm{keV}$ $\mathrm{Cs}^{+}$. The peak oscillation amplitude for the calculation of $\sigma_{0}$ is denoted by $x_{p}$.

matching calculations, using the radius and divergence values measured in the horizontal plane. Subsequent changes in quadrupole polarity did not exactly invert the $x$ and $y$ properties of the beam, so the beam was not precisely symmetric at the source.

First estimates of the required matching section configuration were calculated using a $\mathrm{K}-\mathrm{V}$ envelope integration program written by L. J. Laslett and V. O. Brady. This program represents the quadrupole field by superoosition of a Fourier-Bessel field expansion for each of the five unatching quadrupoles and one of the periodic lenses. The expansion includes only the quadrupole, or $\cos (2 \phi)$, terms with the associated Bessel function nonlinearities. The quadrupole focusing field is linearized during the integration by taking the effective gradient at each $z$ position to be the ratio of the vacuum field calculated at the beam edge to the beam radius. The final tuning procedure used involved measuring the RMS beam radius in each transverse dimension in two consecutive quadrupole gaps, after M5 and Q1. The linear response 
matrix calculated by varying four of the matching elements (usually M2-M5) in the envelope integration program provided a very dependable means of predicting corrected voltages. In most cases, five iterations were sufficient to provide a beam matched to within $10 \%$ envelope variation over the first 4 periods of periodic transport.

\subsection{Emittanc: Measurements}

Because we are using a double-slit emittance scanning method, only one point in the phase space distribution $f\left(x, x^{\prime}\right)$ can be determined per beam pulse. Hence pulse-to-pulse variation and overall reproducibility are important (discussed in section 7.8). In the early stages of the experiment, we had logged the data visually from the oscilloscope traces and moved the slits manually. This made the data analysis very tedious. The HP-85 system was therefore developed to handle the large number of data points (about 1000 per hour, with acquisition times of 1-1.5 hours). Almost all of the emittance data reported here are from the high-perveance, automated data acquisition mode.

\subsection{Data Analysis}

The RMS emittance is formally defined as

$$
\epsilon_{\mathrm{RMS}} \equiv \sqrt{\left\langle(x-\bar{x})^{2}\right\rangle\left\langle\left(x^{\prime}-\overline{x^{\prime}}\right)^{2}\right\rangle-\left\langle(x-\bar{x})\left(x^{\prime}-\overline{\bar{x}}\right)\right\rangle^{2}} .
$$

For a K-V beam, the RMS emittance is identically one-fourth of the actual emittance, and we have chosen to multiply the RMS emittance by this factor of 4 in calculating the emittance of our beam, following Lapostolle [14]:

$$
\epsilon \equiv 4 \epsilon_{\mathrm{RMS}}
$$




\subsubsection{Stability Criterion}

Our experimental procedure is straightforward. We set initial values for the curzent and emittance and measure the evolution of these parameters through the lattice. If the current and emittance are conserved throughout the lattice, we call the conditions "stable"; otherwise the conditions are called "unstable." This criterion has practical utility in the design of an accelerator for ICF roork. The major purpose in identifying the boundary between "stability" and "instability" is to provide relevant information for the design of such accelerators.

\subsubsection{Phase space data presentation}

We present the phase space data in two graphic forms. First, we show the measured intersity contours in phase space, as in Fig. 5.2. These contours are measured at antisymmetry points along the channel, between lenses. At such points, the phase space contours (roughly elliptical in shape) are not upright, but are tilted with respect to the $x$ and $x^{\prime}$ axes as a result of the convergence or divergence of the beam at the measurement point. In order to aid in viewing the contours, this tilt has been suppressed by a linear transformation of the $x^{\prime}$ axis, of the form $x^{\prime} \rightarrow x^{\prime}-a x$ in all plots of this type. The value for $a$ is chose:- to make the average value of the product of $x$ with the new $x^{\prime}$ identically zero: $a=\left\langle x x^{\prime}\right\rangle /\left\langle x^{2}\right\rangle$. This is equivalent to translating the distribution to a symmetry point, except that the beam size retains the value for the antisymmetry point. In addition, we plot the emittance and current associated with the various intensity contours in the following way. We generate sub-distributions from the measured data by successively deleting points with values below certain thresholds in intensity. We then plot both the area occupied by the sub-distributions and the associated $\epsilon(i)=4 \epsilon_{\mathrm{RMS}}(i)$ values against the partial current (i) represented by the sub-distributions, as 
in Fig. 5.6. The area emittance is used only in these plots; the emittance from the RMS reduction is used everywhere else. Although the $i$ vs. $\epsilon(i)$ curves do not reveal aberrations unless they are severe, the contour plots show them quite clearly.

\subsubsection{Intensity parameterization and $\sigma$ values}

The emittance for the full beam current, $\epsilon(I)$, varies somewhat for the high- $\sigma_{0}$ measurements depending on how much "empty" phase space area surrounds the beam-occupied region, because of inclusion of noise in the data. The values for $\epsilon(0.95 I)$ are more stable, and wherever values for the emittance are quoted, the value is for the $95 \%$ core of the beam distribution. This corresponds approximately to $90 \%$ of the beam when both dimensions are accounted for, assuming the distributions in the $x$ and $y$ planes are uncorrelated.

In our summary of maintainable beam intensity we must compare our results with the theoretical work, which has often been summarized in terms of the zero-current phase advance $\sigma_{\circ}$ and the space-charge "depressed" phase $\therefore$ dvance $\sigma$. We quantify the intensity of a beam by the value of $\sigma$ derived for a $K-V$ beam with the same current and emittance as measured for the non $K-V$ beam, with the following justification. For a given $\sigma_{0}$, the parameter determining $\sigma$ in the $\mathrm{K}-\mathrm{V}$ treatment is $\epsilon / I$. (This ratio does not uniquely specify non-K- $V$ beam distributions, but if one scales a given beam distribution to different current, keeping the particle trajectories similar, the quantity $\epsilon / I$ remains constant. This same scaling can be inferred from Eqns. 2.5.) The nonlinear fields of a real beam with space-charge cause a spread in particle oscillation frequencies. However, because the forms of the K-V and RMS envelope equations are identical, the ratio of emittance to current determines the envelope stability for any beam for which the RMS equations are appli- 
cable.

We derive values $\sigma_{100}$ and $\sigma_{95}$ as estimates for the average betatron frequencies of the beam particles using the linear theory in the following way. We use the emittance $\epsilon_{100} \equiv \epsilon(I)$ for the total beam current $I$, and also the emittance $\epsilon_{95} \equiv \epsilon(0.95 I)$ for the most intense $95 \%$ of the beam current to derive depressed phase advance values $\sigma_{100}$ and $\sigma_{95}$, respectively, for $K-V$ beams with a ratio of emittance to current equal to $\varepsilon_{100} / I$ and $\epsilon_{95} / 0.95 I$. We find that these two values are always close to each other, as shown in Fig. $\mathbf{5 . 1 1}$ and Table 5.1. 
Chapter 5: Beam Measurement Results

In the preceding chapters we have discussed the experimental procedures used and given a framework for analysis of the data. We present below the results of our measurements, divided into two major categories:

- results for lattice strength up to the onset of detectable growth of beam emittance $\left(59^{\circ} \leq \sigma_{0} \leq 88^{\circ}\right)$.

- results for the range $\sigma_{0}>88^{\circ}$, over which collective effects place a measurable lower bound on the beam emittance for a given current; or, conversely, an upper bound on the current that does not cause emittance growth.

We will informally use the desiriptions "low- $\sigma_{0}$ " for the first of these regions, and "high $-\sigma_{0}$ " for the second. Beams with $\sigma_{0}$ below $59^{\circ}$ are stable as checked at $\sigma_{0}=45^{\circ}$, but these data are of very limited extent and of less interest and hence are not discussed here. Sources of error are discussed in Chapter 7.

\subsection{Results for $\sigma_{0} \leq 88^{\circ}$}

\subsubsection{Focusing aberrations}

The SBTE source is a solid-state source and produces a relatively uniform current density (see Fig. 5.1). There is a small spherical aberration, a deviation from linear optics, of only about $20 \%$ of the intrinsic width of the source distribution, detectable using the horizontal dimension source diagnostics (not shown). The only apparent consequence of this aberration is the higher current density at the edges of the beam as it exits the injector, as shown in Fig. 5.1. In general, aberrations in the focusing of the beam will result in distortions of the phase space contours from elliptical shapes, either by making the contours more square in shape or by distorting the contours 
Scan over source with $2 \mathrm{~mm}$ hole in wheel aperture

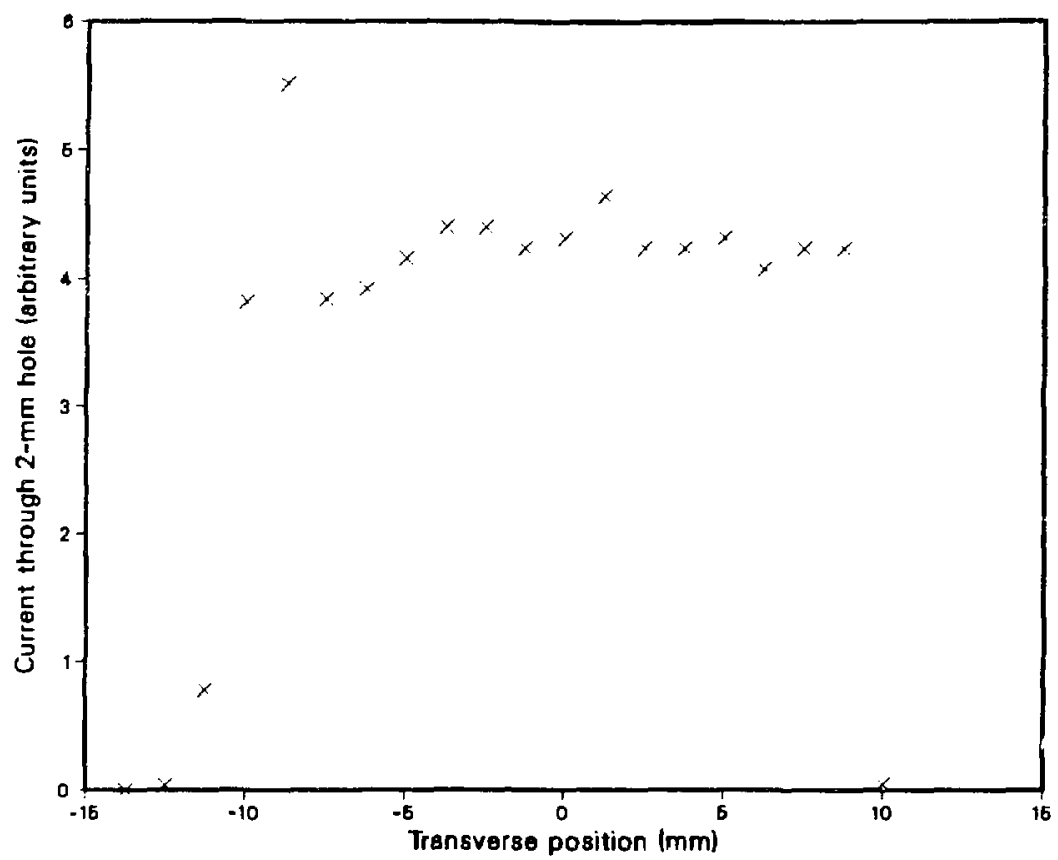

x82 $855-1761$

Figure 5.1: SBTE injector current density profile, measured using a 2-mm diameter aperture and scanning across the source at approximately the vertical center. The observed current density is relatively uniform over the beam diameter. The high point at the left of the figure is reproducible, and may be a result of the imperfect focusing of the injector on the beam. For other values of height that we could check, the beam profile was similar. 
into an S-like shape. We will refer to aberrations as a property of the beam distribution (caused by aberrations in the overall focusing field), as well as being a property of the focusing field itself. More serious aberrations in the phase space distribution of the beam were noted downstream of the matching section. We show three sequences of phase space contours in Fig. 5.2. The four rows show measurements at, respectively, quadrupoles Q4, Q35, Q59, and $\mathrm{Q} 80$, in the horizontal dimension. For column (a), $\sigma_{0}=59^{\circ}$. The minor distortion seen at $\mathrm{Q} 4$ is not visible in the downstream measurements. We infer that the slight S-like shape of the contours as measured at Q4 is due either to matching section aberrations or to tile source distribution. In contrast, phase space contours for one $\sigma_{0}=83^{\circ}$ matching attempt are shown in column (b) of the same figure. The distortion of the contours at Q4 is much more severe for $\sigma_{0}=83^{\circ}$ than for $\sigma_{0}=59^{\circ}$. The aberrations again are not evident in downstream measurements, although for the phase space distribution as measured at Q35, the contours surrounding most of the beam enclose an area appreciably greater than for the corresponding $\sigma_{0}=59^{\circ}$ case. The aberration as measured at $\mathrm{Q} 4$ was visibly diminished by weakening the M1-M2 doublet, varying the other matching elements to maintain a matched beam (see Fig. 5.2c). In this figure, the voltages on the elements M1-2 were about 15\% lower than for Fig. 5.2b.

\subsubsection{Beam loss bounds}

We could not measure the current of the beam as it exits the source, because of the restricted clearance and the geometry of the source housing. The first point along the lattice at which we could insert a deep cup for current measurement was after M5, the last matching quadrupole. We also had SFC's along the lattice at $\mathrm{Q} 2, \mathrm{Q} 36$, and $\mathrm{Q} 60$. For $59^{\circ}<\sigma_{0}<83^{\circ}$, with well-matched initial conditions, no loss of beam was detected between M5 and Q82, within 

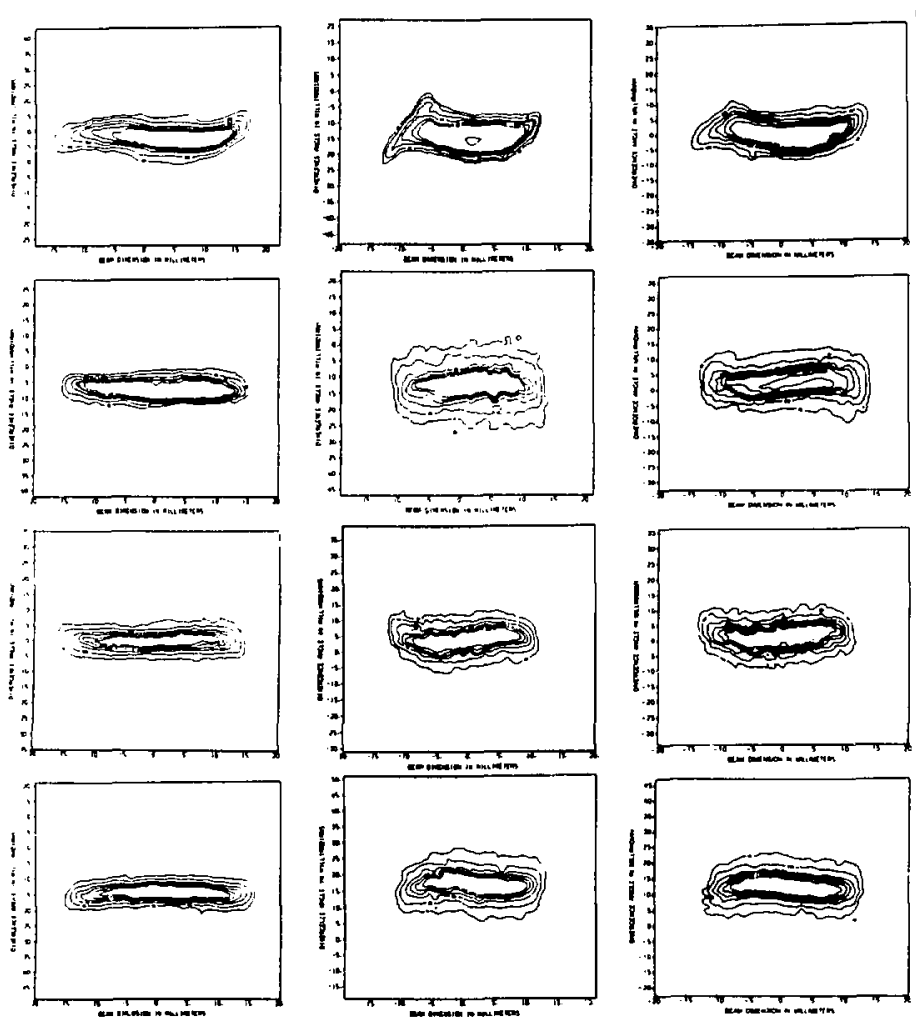

(a)

(b)

(c) $\times 161865-1874$

Figure 5.2: Focusing aberrations downstream of the matching section. The four rows are for measurements made, respectively, downstream of quadrupoles Q4, Q35, Q59, and Q80. (a) A sequence of measurements along the lattice for $\sigma_{0}=59^{\circ}$. The aberration after $Q 4$ is very small, and washes out during further transport. (b) An early set of measurements for $\sigma_{0}=83^{\circ}$. The aberration at Q4 is much worse than for (a), but washes out during further transport. In this process, however, the low level contours in phase space appear to become diluted. (c) When we weaken the M1-2 doublei of the matching section, maintaining a matched beam by varying the other matching elements, the aberration measured for $\sigma_{0}=83^{\circ}$ at $\mathrm{Q4}$ is diminished. The broadening in the low-level contours in the downstream measurements also is not as pronounced. 
the experimental precision of about $2 \%$, by direct measurement on the cups. Fig. 5.3 shows DFC traces after M5 with various pulse-lengths compared to the DFC trace after Q82. The figure shows the discrete downward steps in current due to the light ion velocity difference from the cesium ions. The direct response of the DFC at M5 to the cesium component is the same, within about $\sim 3 \%$, as for the DFC at Q82. We expect the measured current at Q82 to be 2-3\% lower than at injection as a result of the Marx pulse droop (see section 4.2), and about 1-2\% lower from background gas collisions (see section 6.3). By direct comparison between these identical cups, we are able to detect any beam loss of more than about $0.3 \mathrm{~mA}$, but we find no beam loss at this level.

For the unattenuated SBTE beam, after we shortened the injector to raise the gun current, we found that the current delivered into the DFC at Q82 was not absolutely constant, but showed a slight dependence on $\sigma_{0}$. The deviations in measured current were at the limit of experimental uncertainty, at the $2 \%$ level. The current measured at the end of the lattice for our best $\sigma_{0}=59^{\circ}$ match was $15.2 \mathrm{~mA}$, while for the best $\sigma_{0}=78^{\circ}$ match, we measured $15.5 \mathrm{~mA}$. Although the current measured at the entrance to the transport channel varied by about the same amount between the two cases, the slightly higher current may be due in part to the phenomenon found by Brewer [18]. A boundary layer of particles at the periphery of the source is focused improperly by fields distorted from the ideal by imperfect boundaries. These particles pass through the beam and penetrate outside the bulk beam distribution. If the beam clearance in the aperture is too small, these particles will be lost against the quadrupoles. But if the aperture is increased (by increasing the focusing field, in this instance), then the particles will be retained. We took care during construction of the ion source to minimize any gaps between the surface of the ion emitter and the focusing electrode bound- 


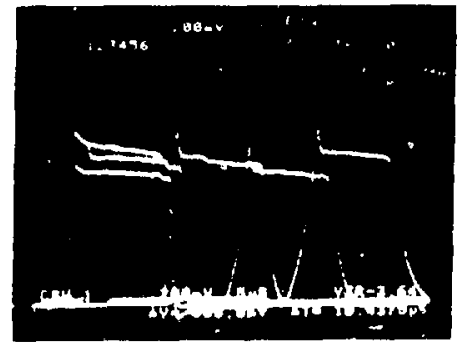

(a)

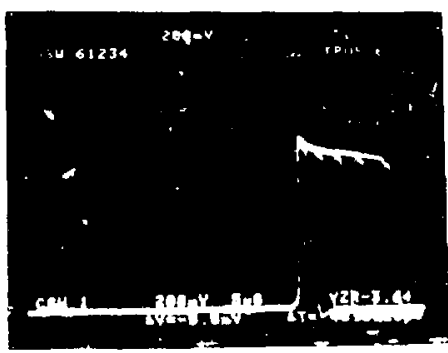

(b)

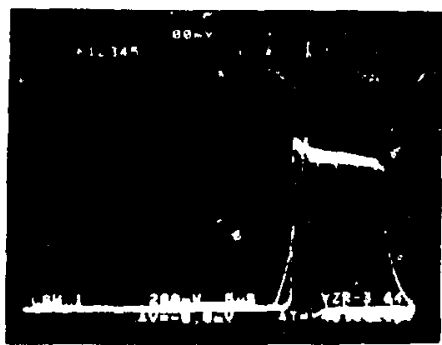

(c) $\times 13 B \quad 864-2707$

Figure 5.3: (a) Faraday cup traces along the lattice, showing the differences in the direct response to essentially equal bearn currents. (b) Deep Faraday cup traces with short fall-times (at M5), showing the separation of the light ion component from the $\mathrm{Cs}^{+}$component in the small step at the trailing edge of the beam pulse before the main pulse ends. (c) Overlay of the $\mathrm{Cs}^{+}$ component as measured at M5 and at Q82 using identical DFC's. The long fall-time at Q82 prevents observation of the undershoot at the tail of the pulse. The equality of $\mathrm{Cs}^{+}$component at either end of the lattice to within about $2 \%$ (the level of the beam loss from background gas interactions and beam debunching from the Marx generator output droop) may be seen from the oscilloscope traces. The effect of the longitudinal electric field is apparent from the elongation of the beam ends. 
aries, but a small separation was necessary to avoid unnecessary conduction of heat away from the source. Two parcent of the beam current corresponds to a boundary layer only 0.005 inches in width around the periphery of the 1inch diameter source; this fraction of the beam could easily be subject to edge aberrations in the injector and br very difficult to identify in measurements on the beam.

Somewhat later, we provided for measuring charge accumulation on the quadrupoles, and we observed small beam-coincident currents. At this time, the injector grids had been altered, and the diagnostics needed for careful matching of the beam had been removed, so that we had no way of generating a well-matched beam. The ion beam spill current necessary to induce this signal, assuming a secondary electron gain factor of 15 (adjusted upward from 12 to make a crude allowance for field-enhanced and grazing incidence emission from the quadrupole surfaces), would have resulted in a $0.2 \mathrm{~mA}$ beam loss over the entire lattice if the loss were uniform in $z$. This was measured only at one location, half-way down the lattice and with a beam that had wul been carefully matched. The signal was independent of pressure for low pressures, and thus was not due to background gas interactions. Loss of "translaminar" particles from the bear would provide a similar signal. These observations are from measurements at the limit of experimental uncertainty, and we believe that there is no collective mechanism involved to cause beam loss, given the existence of the mechanism described by Brewer.

\subsubsection{Beam envelope measurements}

We show some beam profile sequences for $\sigma_{0}=59^{\circ}$ and $\sigma_{0}=83^{\circ}$ in Fig. 5.4. As the strength of the lattice is raised, and the aberrations discussed in section 5.1.1 show up in the phase space measurements, the profile of the beam is also affected. By weakening the M1-2 doublet, the beam profiles are 
smoothed near the injector. The beam profile is much smoother at the end of the lattice, as a result of dispersion of the space-charge oscillations.

Following the RMS envelope formalism, and denoting the RMS value of $x$ as $\tilde{x}$, we plot $2 \tilde{x}$ for the beam profiles of Fig. $5.4 \mathrm{c}$ and $d$, versus position along the lattice. The results are shown in Fig. 5.5. The value calculated from from the envelope equations is about $12 \mathrm{~mm}$. (The measured beam size agrees very well with calculation using the envelope equations, as shown in Table 5.1 below. A set of data spanning the range of $\sigma_{0}$ used in these experiments from measurements at the end of the lattice is shown in Table 5.1 on page 82.) The profile data show initial transient oscillations quite clearly. Calculations of the frequencies of the simple envelope oscillation modes for the constantfocusing model are given in [40], and we have included some information in Ch. 2. The result for the symmetric breathing mode (apparent in Fig. 5.5 for the RMS radius of the beam) in terms of $\sigma$ and $\sigma_{0}$ is given in Eqn. 2.9

$$
k^{2}=2 \sigma_{0}^{2}+2 \sigma^{2},
$$

where $k$ is the "wave-number" of the envelope oscillation, or $360^{\circ} / \lambda$, where $\lambda$ is the number of focusing periods in one wavelength of the oscillation. For this beam, $\sigma_{0} \simeq 83^{\circ}$ and $\sigma \simeq 16^{\circ}$. The resulting wavelength of the envelope oscillation is very nearly three lattice periods, in very good agreement with the data. When we replaced the $45^{\circ}$ parallel-wire grid used to terminate the injector with a 0.125 -in cell-size honeycomb grid, the beam profile near the injector became much smoother, so we attribute the major part of these beam oscillations to perturbations due to the parallel-wire grid.

Although the RMS envelope oscillation is substantially dissipated by the time the beam has reached Q73 (see Fig. 54), the . d moment of the beam distribution persists, with the beam still "sloshing" iom side to side as it progresses down the channel. This mode of oscillation is visible also in 

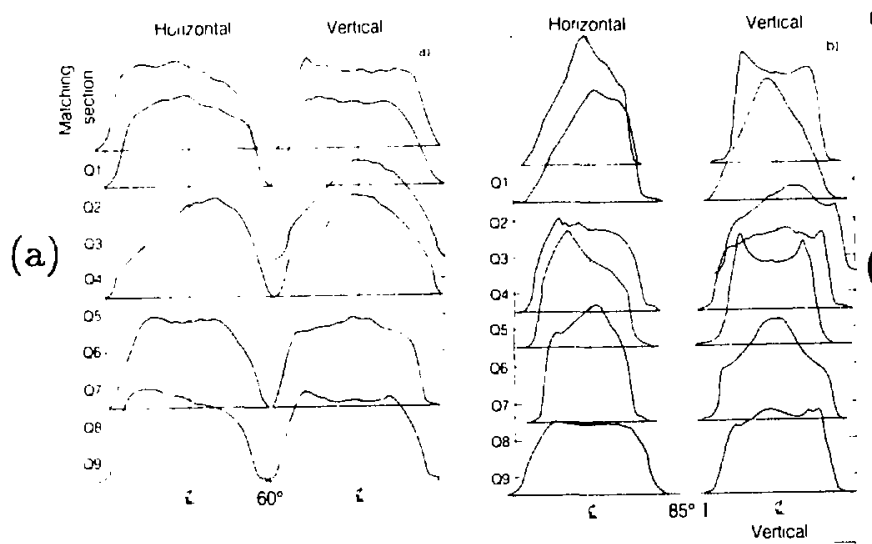

Figure 5.4: (a) For $\sigma_{0}=59^{\circ}$, the beam shows both a side-toside "sloshing" mode and a nonuniform breathing mode. (b) For higher lattice strength $\left(\sigma_{0}=83^{\circ}\right)$, the perturbation appears much more severe than for $\sigma_{0}=59^{\circ}$. (c) When we weakened the M1-2 doublet, the perturbations in the profile grew weaker. (d) By the time the beam had reached the end of the iattice, the profile perturbations had smoothed considerably, although the sloshing mode is still visible, as it was in the third moment data in Fig. 5.5.

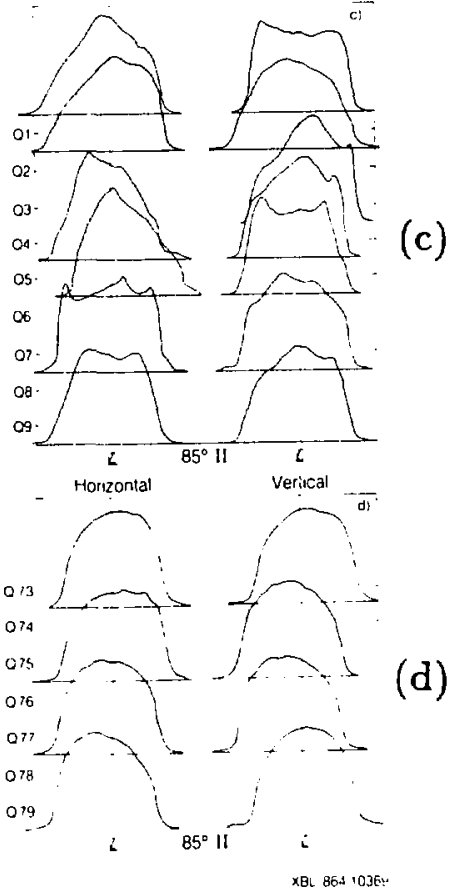




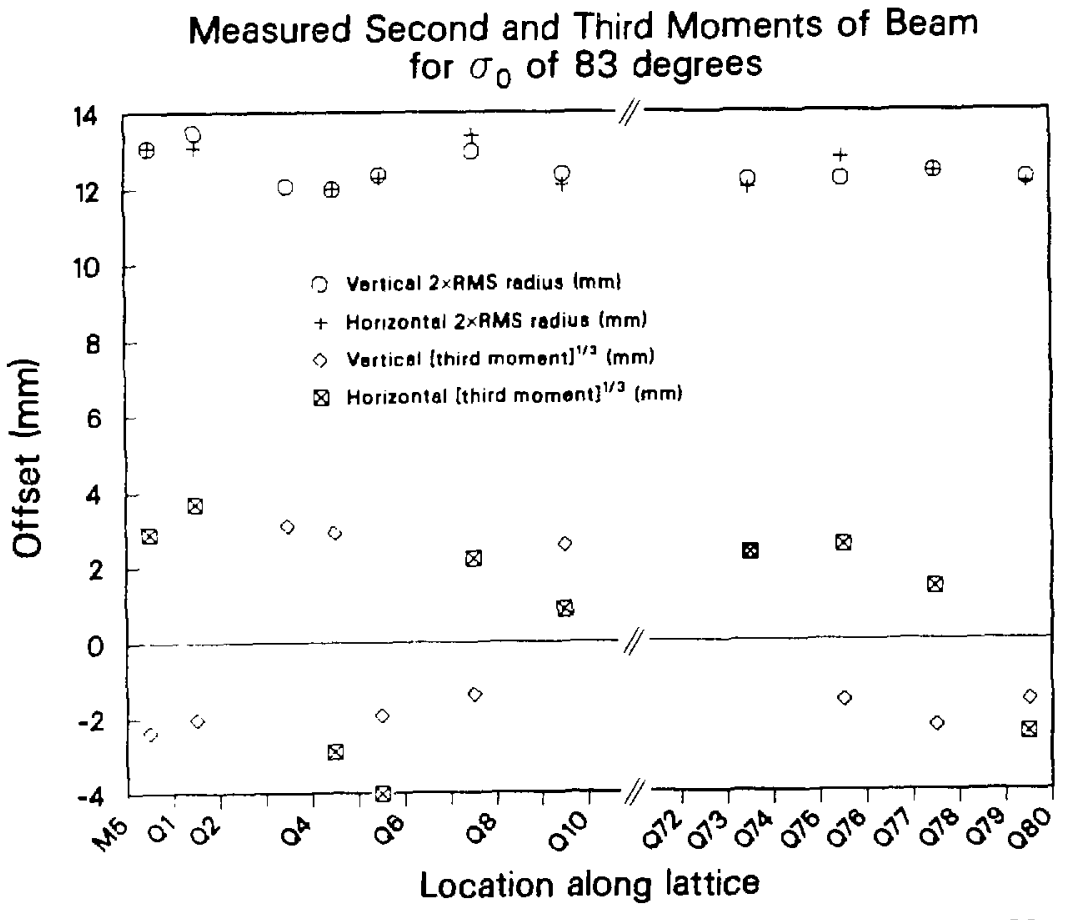

Figure 5.5: The points near the top of the figure show the measured RMS beam radius along the lattice for $\sigma_{0}=83^{\circ}$. The envelope oscillation in the two planes is almost exactly in phase, so that the envelope oscillation with these initial conditions is in only one mode. The wavelength, $\lambda$ in units of focusing periods, calculated from the smooth focusing model is such that $\left(360^{\circ} / \lambda\right)^{2}=2 \sigma_{0}^{2}+2 \sigma^{2}$. For $\sigma_{0}=83^{\circ}$ and $\sigma \sim 16^{\circ}, \lambda$ given by this formula is about three focusing periods, which is in good agreement with the measurements. The third moment of the beam distribution is plotted along the lower portion of the figure. The period of the oscillation is in reasonable agreement with the value $360^{\circ} / \sigma_{0}$, as it should from constant-focusing calculations for $\sigma$ much less than $\sigma_{0}$. 
the profiles for M5-Q9, and we have included it in Fig. 5.5 by plotting the third moment of the beam profile along the lattice. We made no detailed comparison of the frequency of these oscillations with theoretical predictions, but we note that in the limit of a cold beam, there is a sextupole-symmetry oscillation with a non-zero third moment, with frequency degenerate with $\sigma_{0}$ [15]. The wavelength of such an oscillation for $\sigma_{0} \sim 83^{\circ}$ is about four foctusing periods, which is consistent with the observations in Fig. 5.5.

\subsubsection{Phase Space Measurement Results}

For $\sigma_{0}<88^{\circ}$, we saw no growth of emittance along the lattice for the current and emittance range experimentally accessible. $R$ ، sults for many measurements in the range $59^{\circ} \leq \sigma_{0} \leq 83^{\circ}$ showed a nearly constant emittance downstream from the matching section. There is a slight consistent decrease in the RMS emittance for $95 \%$ of the beam particles as the beam passes through the lattice. The decrease is in the range 5-10\%, and may be due in part to some systematic effect, such as achieving better slit alignment near the end of the lattice. Loss of the outer $2 \%$ of the beam could have a similar result. The slope of the $i$ vs. $\epsilon(i)$ curve, which is a measure of the phase space density of the beam, remains nearly unchanged. The emittance versus current for the highest-intensity $\sigma_{0}=59^{\circ}$ beam we could obtain from our source is shown in Fig. 5.6. When plotted on linear scales, the bulk distribution has a nearly linear current vs. emittance relationship. In the tails of the distribution, the $I$ vs. $\epsilon$ curve breaks over, reflecting the low density of the tail.

As we attempted to match the beam into stronger lattices, the emittance measured after Q4 grew larger, because of the aberrations mentioned in section 5.1.1. The emittance was constant or decreased alightly along the lattice at the higher value for $\sigma_{0} \leq 88^{\circ}$, and we attribute the higher emittance down- 


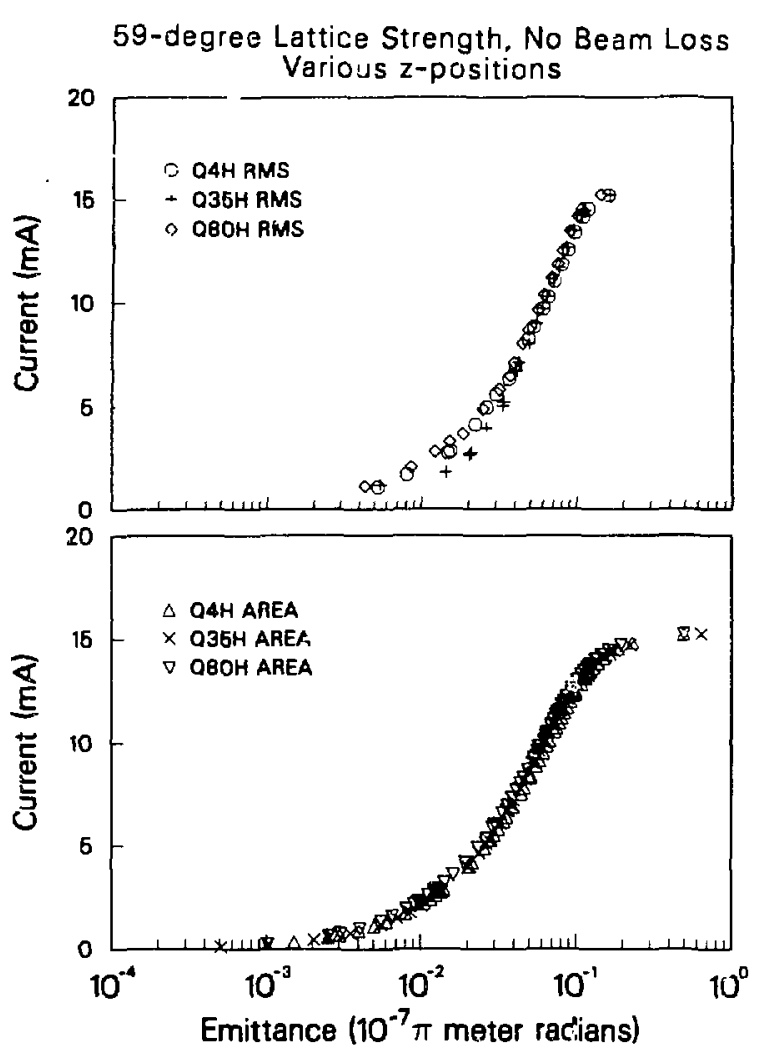

XCG $864-7154$

Figure 5.6: Plot of $i$ vs. $\epsilon(i)$ for $\sigma_{\mathrm{D}}=59^{\circ}$. (a) RMS analysis of emittance, and (b) Area emittance. The distribution is almost linear for $i / I<0.9$ when plotted on linear scales. In these and the succeeding figures 5.7 and 5.8 , there is no perceptable change in the overall emittance. In the actual phase space data, however, there is a small tail of ions, with a density on the order of $1 \%$ of the peak value of the distributior, sich forms outside the main beam. This is to be expected from realistic distributions, as a result of thermal tails in the distribution. 
stream of the matching section in these cases to the matching section fields. Figs. 5.7 and 5.8 display the effect on the beam of raising the lattice strength. The area emittance curves show a growth for $\epsilon(I)$ as $\sigma_{0}$ rises, but not much variation along the lattice.

The only measurements we made using a lattice weaker than $59^{\circ}$ were for $\sigma_{0}=45^{\circ}$. These measurements were all made during the manual data acquisition period, with poor resolution, but we saw no evidence of emittance growth. This is in accordance with our belief that one cannot get into trouble by weakening the lattice if the beam is stable for $\sigma_{0}=59^{\circ}$.

At about the $\sigma_{0}=88^{\circ}$ level, with $\sigma \sim 16^{\circ}-18^{\circ}$, a small, persistent current loss began to occur, of about $4 \%$. This lattice strength is in fact the stability limit for the most intense beam we could produce. Even a $6 \%$ increase in lattice voltage, corresponding to a $\sigma_{0}$ of about $94^{\circ}$, resulted in a $30 \%$ current loss at the Q82 DFC and a rapid rise in the emittance of the beam, as shown in Fig. 5.28. This marks the presently detectable onset of collective instability for space-charge dominated beams. The variation with lattice strength of the beam current transmitted to the DFC downstream of $Q 82$ for $\sigma_{0} \simeq 90^{\circ}$ is shown in Fig. 5.9. The beam was matched for $\sigma_{0}=88^{\circ}$, with a matched beam radius between quadrupoles of approximately $12 \mathrm{~mm}$. The drop in current for low quadrupole voltage is due to mismatch of the beam.

\subsection{Discussion of Results for $\sigma_{0} \leq 88^{\circ}$}

\subsubsection{Low-emittance limits}

Theoretical predictions based on K-V model beams have indicated several mechanisms for emittance growth. Analogous to the $\sigma_{0}=180^{\circ}$ single particle resonance with the focusing lattice, there are higher-order resonances possible for $\sigma_{0} \geq 180^{\circ} / n$ for $n \geq 2$. The envelope instability for $\sigma_{0} \geq 90^{\circ}$ is one such mode, for $n=2$. The various oscillation modes for the K-V beam 

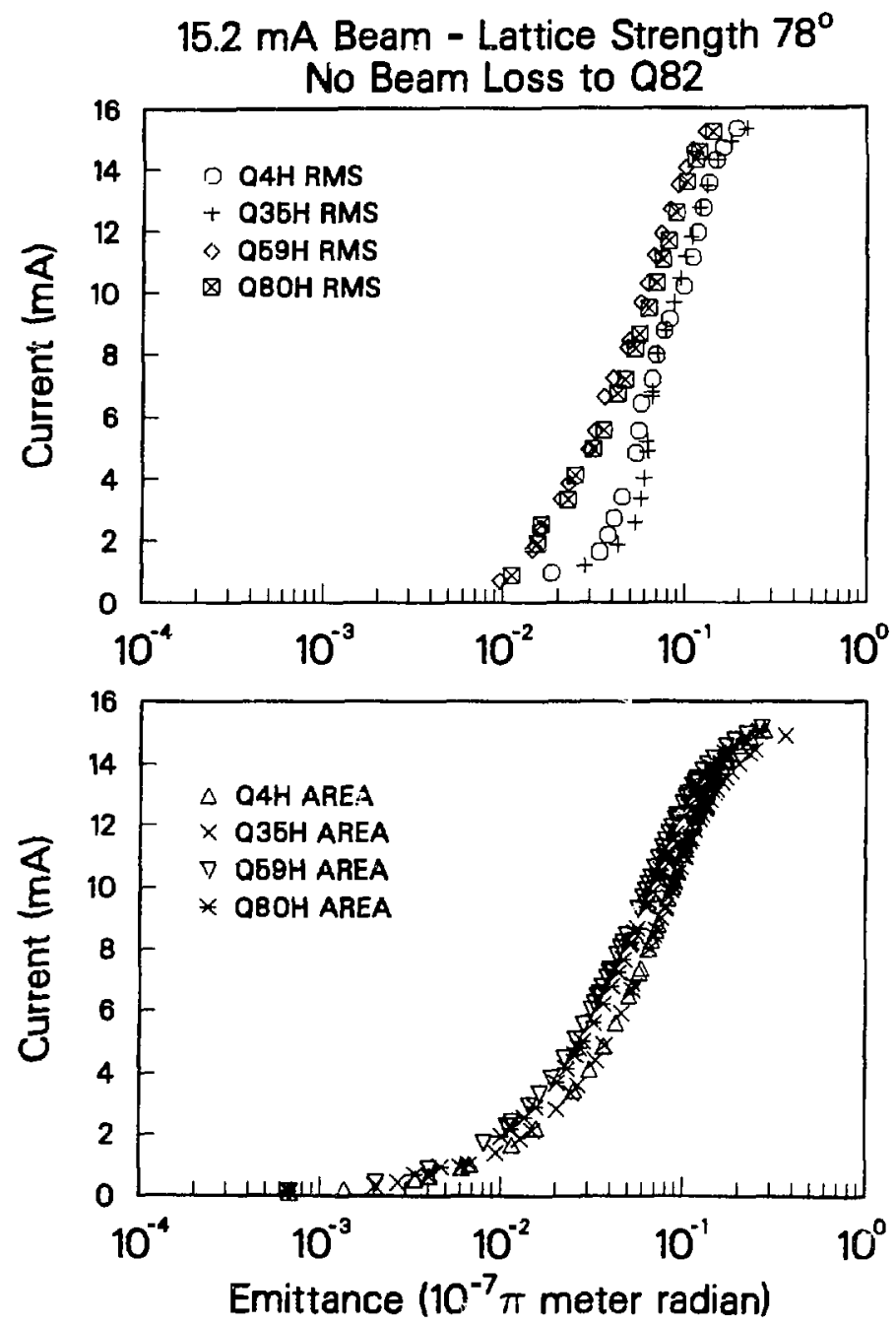

XCG 864-7161

Figure 5.7: Integrated area and RMS emittances in the horizontal dimension for $15 \mathrm{~mA}$ beam, $\sigma_{0}=78^{\circ}$. 

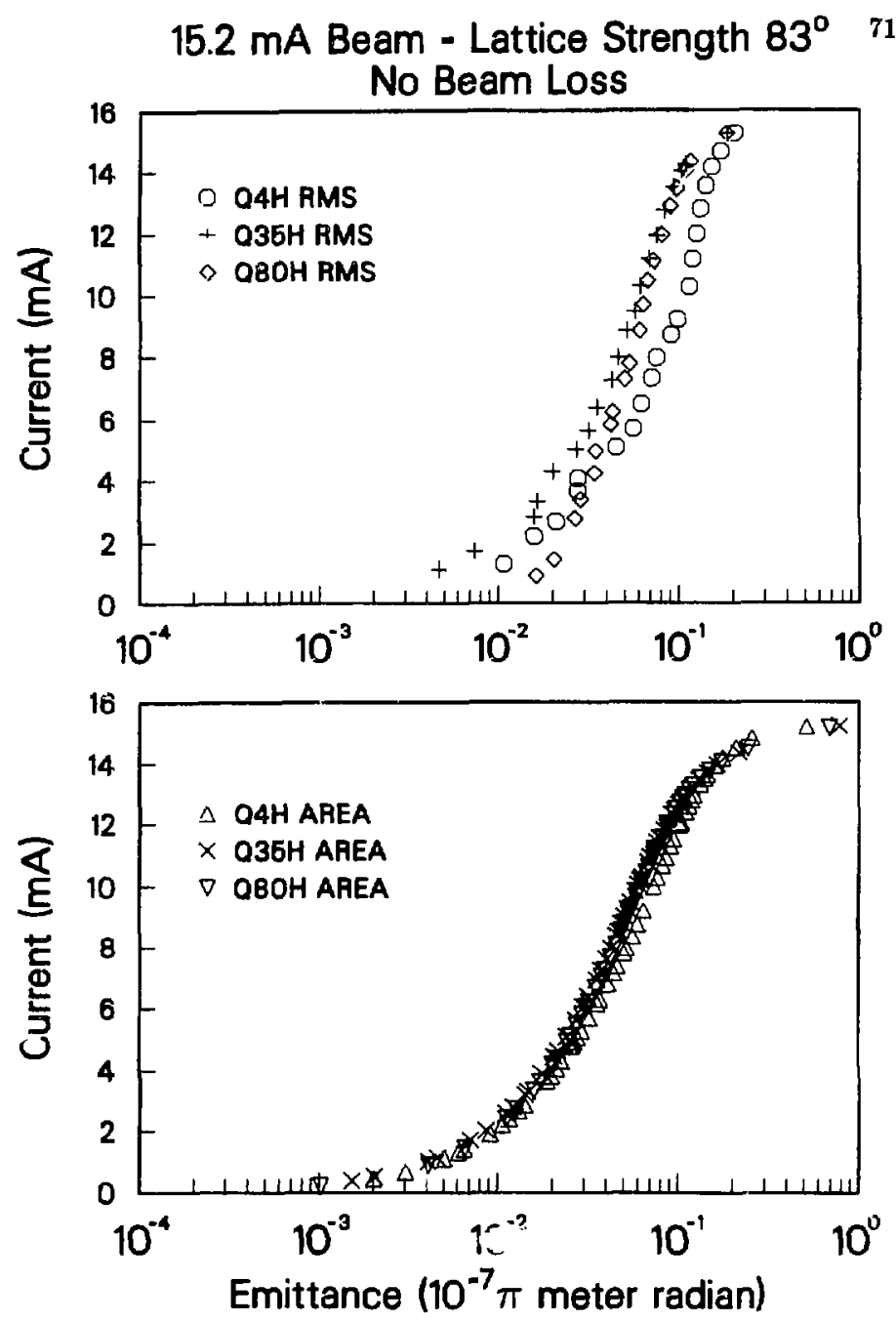

XCG $864-7162$

Figure 5.8: Integrated area and RMS emittances for $15 \mathrm{~mA}, \sigma_{0}=83^{\circ}$. The phase space contours are particularly distorted for the Q4 measurements (see Fig. 5.2c), which results in higher values for the RMS emittance there than for the downstream measurements. 
Current Transmitted for Nominal $88^{\circ}$ Match

Showing High Strength Current Falloff

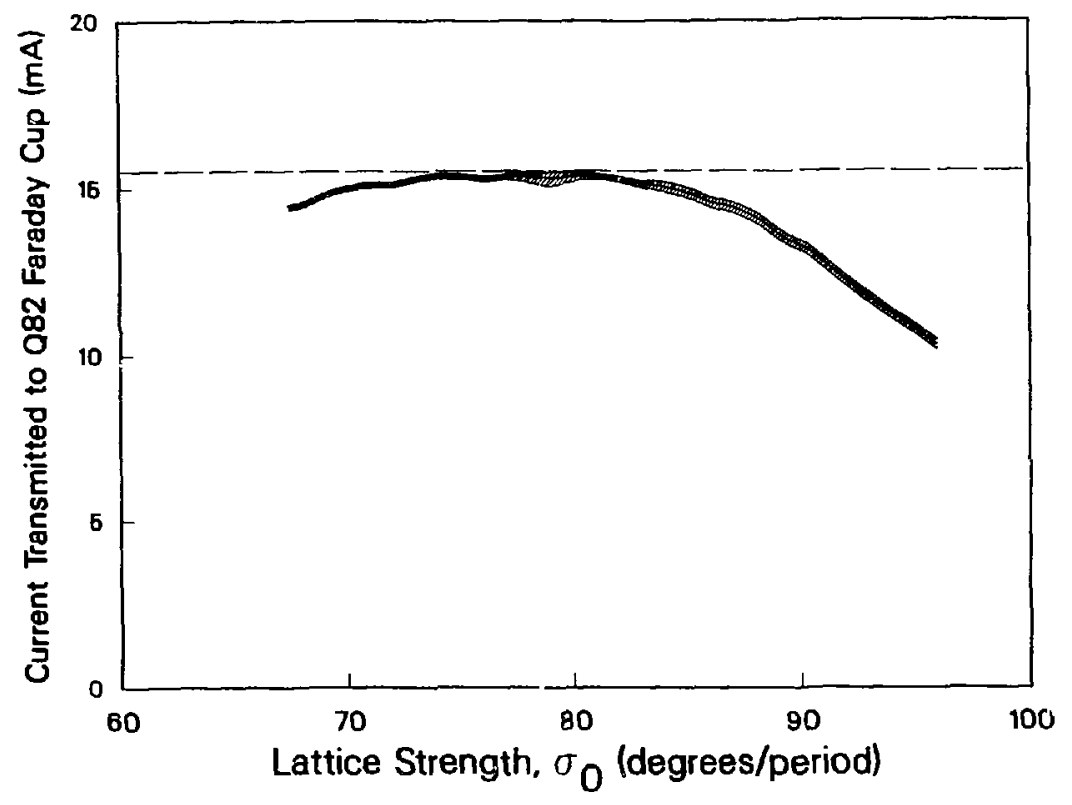

$x \operatorname{CG} 864-7166$

Figure 5.9: Current transmitted through the lattice for $\sigma_{0} \sim 90^{\circ}$, showing the falloff in transmitted current for $\sigma_{0} \sim 90^{\circ}$. The beam loss at the low lattice strength end of the plot is due to mismatch of the beam. 
are characterized by perturbed electrostatic potential functions expressible in terms of finite polynomials $n x$ and $y[15,16]$. The various modes were classified by the order of the polynomials. (For example, for a "third-order" mode, the highest-order term in the perturbed potential is $x^{2} y$ or $y^{3}$.)

In addition, there is the possiblity that in a beam with a high degree of order, such as a K-V distribution, negative-energy modes of oscillation can exist and become degenerate in frequency with positive energy modes. The resulting energy transfer between modes can result in emittance degradation, in a way analogous to the two-stream instability in plasmas [17].

As a result of such calculations, there had been concern that the existence of $n=3$ ("third-order") modes could require that $\sigma_{0}$ be limited to no greater than $60^{\circ}$, and that the threshold values of $\sigma / \sigma_{0}$ for fourth- and higher-order mode instability (about 0.4 ) would limit the space-charge depressed phase advance for $\sigma_{0}=60^{\circ}$ to $\sigma=24^{\circ}[20]$.

Our work shows no evidence of any unstable behavior for the lowest accessible values of $\sigma$ for lattice strength up to about $\sigma_{0}=88^{\circ}$. There has, however, been some work on the limiting effects caused by interaction with induced charge on the lattice [41]. This effect may prove to be the practical limit on low-emittance beam transport. Our relevant measurements are discussed in section 6.2 .

\subsubsection{Plasma-like behavior}

In the low- $\sigma_{0}$ limit, an alternating gradient lattice may be represented by a smooth-focusing equivalent for the purpose of calculating certain parameters of the beam. The smooth focusing model is physically equivalent to a fixed uniform background charge density of the opposite sign to that of the beam particies, and so the beam looks very much like a plasma. In this model, the KK-V beam, as well as a Maswellian beam for low transvese temperature, has 
a circular cross-section and a uniform particle density. The projection of this distribution onto one spatial dimension gives a locus of current vs. $x$ which is elliptical. Maxwellian solutions with a non-zero beam temperature have tails in the distribution in real space, with a scale length given by the Debye length appropriate to the density and transverse velocity spread of the beam. Within the plasma framework, the physical interpretation is simple. For a cold beam, the restoring well is almost neutralized by the beam particles over the bulk of the beam cross sect:on, and the residual forusing field inside the beam is very small. The beam edge occurs at the location where the restoring well poiential becomes comparable to the thermal energy of the particles. The beam density drops nearly to zero over a distance of about $2 \lambda_{D}$. For cold beams, the particle density is neariy constant until this sheath is reached. The result is an elliptical 1-d current profile over the bulk of the beam, with smali tails at the edges provided by the sheath particles.

We show in Fig. 5.10 a comparison between some of our measured beam profiles for $\sigma_{0}=59^{\circ}$ near the end of the channel and smooth-focusing solutions calculated for Maxwellian distributions with the same current and RMS emittances as measured for the real beams. We take the distributions to be of the form

$$
f_{M}(x, y)=n_{0} \exp \left(-\frac{\kappa\left(x^{2}+y^{2}\right)-e \phi(x, y)}{k T}\right)
$$

where $\kappa$ is an effective spring constant for the lattice, $\phi(x, y)$ is the beam self-potential, and $k T$ is the transverse temperature of the beam, in joules. We numerically integrated solutions to the Poisson equation, assuming a Maxwellian particle distribution. The physical extent of the tails in Fig. 5.10 is in reasonable agreement with the calculated value. The beam temperature, 
Comparison of measured beam profiles with smooth-focused Maxwellian calculation for $\sigma_{0}=60^{\circ}$

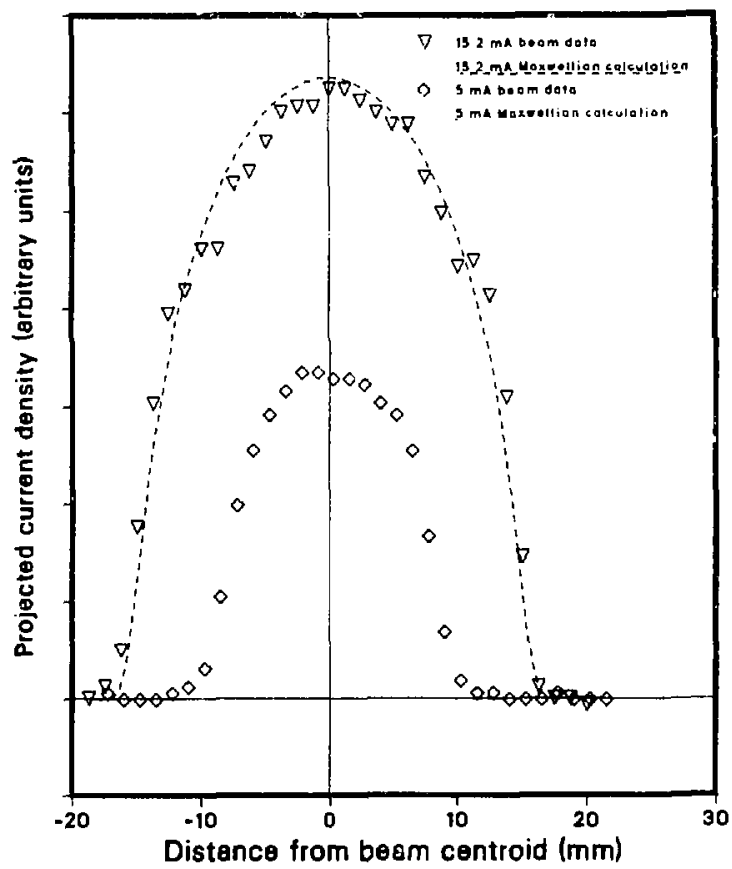

XBL 865-1837

Figure 5.10: Transverse density distritiution in $x$ (measured by a traversing slit) for the $15-\mathrm{mA}$ beam, and for a $5-\mathrm{mA}$ beam, both for $\sigma_{0}=59^{\circ}$. The dotted lines represent calculated profiles for Maxwellian transverse beam distributions in a smooth-focusing lattice. Small tails in real space due to the thermal tails of the distributions are visible. The calculated Debye length is about $0.7 \mathrm{~mm}(k T / e \sim 2.3 \mathrm{eV})$ for the $15-\mathrm{mA}$ beam and $1.2 \mathrm{~mm}(k T / e \sim 6.4$ $\mathrm{eV)}$ for the 5-mA beam. 
calculated using the formula

$$
T(\mathrm{eV})=\frac{m \tilde{v}^{2}}{e}
$$

where $\bar{v}$ is the local $x$ or $y$ RMS velocity of the phasa space distribution at the beam centroid, is $2.3 \mathrm{eV}$ for the $15-\mathrm{mA}$ beam. This corresponds to a Debye length for the beam of about $0.7 \mathrm{~mm}$. We also attenuated the beam near the source and repeated the profile measurement. The calculation for the resulting $5-\mathrm{mA}$ beam is also shown in Fig. 5.10, using the same emittance as for the unattenuated beam. With respect to the 15-mA beam, the 5-ma beam is smaller, its temperature is higher (about 6.4 eV), and $\lambda_{D}$ is larger (about $1.2 \mathrm{~mm}$ ). The wire separation for the harp monitor used to make the measurements is 0.05 inches $(1.25 \mathrm{~mm})$, so that the relative uncertainty in the sheath thickness is large.

\subsubsection{Dependence on source distribution}

Strūckmeier, Klabunde, and Reiser [42], arguing from the general conservation of transverse energy, and supported by simulation results, have shown that a very rapid change in emittance can occur as a result of charge redistribution from an initially ill-matched condition. The change in transverse kinetic energy of the particles balances the electrostatic energy change of the beans. These ideas have been developed further by Wangler [43] and Anderson [44].

In these works, it has been shown that in the low-o limit, with linear external focusing, the beam will distribute its spare charge approximately uniformly in configuration space. If the source provides a distribution of current that is too strongly peaked toward the axis, the charge will subsequently spread out as the distribution relaxes. The in itial electrostatic energy 
is higher than the final, and the difference shows up in the random transverse energy of the bram, causing the emittance to rise. An inverse situaion can occur for initially hollow beam distributions.

While we have seen no collective increase in emittance for $\sigma_{0}<88^{\circ}$, there have been results from Klabunde [23] at GSI in Darmstadt, Germany, indicating that for $\sigma_{0}=60^{\circ}$, and an initial beam current and emittance corresponding to our lowest emittance conditions $\left(\sigma \sim 7^{\circ}\right)$, but with an initially Gaussian distribution of charge in configuration space, the beam emittance grows significantly within the first few periods of transport, a distance short compared to a plasma period. It is probable that this effect is due to the nonuniform initial beam distribution, as described above. The GSI results are somewhat clouded, however, in that the lattice is magnetic, and the experiments are made using a long-pulse plasma source, which results in significant background gas reutralization as the pulse progresses. To extract data without neutralization perturbations, they use measurements only early in the pulse.

For the SBTE, we have no recent measurements of the source emittance to compare with that measured after Q4. However, as mentioned in section 5.1.1, the SBTE source produces a relatively uniform current density (see Fig. 5.1), so that this rapid increase in emittance should have little driving energy. In addition, our early measurements of emittance (using the original aluminosilicate coatings) gave about the same value as later measurements downstream.

\subsection{Results for $\sigma_{0} \geq 88^{\circ}$}

It was expected that the regime of lattice strength $\sigma_{0} \geq 90^{\circ}$ would be unsuitable for intense beam transport, because of instability in the envelope equations $[20,40]$. Nonetheless, in this thesis investigation we explored this 
region of parameter space and discovered behavior which to date has no satisfactory theoretical description (a semi-empirical conjectured description will be discussed later, in sec. 5.5).

The analytical work on the stability of the envelope equations is directly relevant for non-K-V beams. Because the K-V and RMS envelope equations are identical in form, they have the same stability properties. Instability in RMS radius will result in a large portion of the beam attaining a large displacen ent within the bore, with subsequent beam loss. The regions of envelope stability and instability are included in Fig. 5.11, along with the final results of our beam measurements in terms of stable limiting values of $\sigma$ and $\sigma_{0}$.

We found no evidence of collective beam interaction for $\sigma_{0} \leq 83^{\circ}$. At $\sigma_{0}=88^{\circ}$, a small beam loss occurred, with no effect on the beam emittance. This marks the first onset of collective beam effects in the course of our measurements. In contrast, for $\sigma_{0}>90^{\circ}$, the unattenuated beam from our source is violently unstable, and we must greatly decrease the contribution of spare-charge forces with respect to the emittance in order to ensure stability. To aid in determining the high- $\sigma_{0}$ stability boundary, we installed a threegrid emittance spoiler in place of the single parallel-wire grid terminating the injector. The new grid assembly attenuated the beam current to $10.3 \mathrm{~mA}$, because of the greater number of less transparent grids in the beam path. Because the first of the grids was coarser in structure (a hexagonal grid of 0.125-inch cell size) than the 0.05-inch spacing parallel-wire grid we had had in place, the output emittance rose to about $2 \times 10^{-7} \pi$ meter radian from the former $1.4 \times 10^{-7} \pi$ meter radian, even with no external bias applied to the prids. (See Appendix C for the dependence of the emittance on the grid geometry.) 


\section{Experimental limits on beam s'ability in terms of $\sigma$ and $\sigma_{0}$}

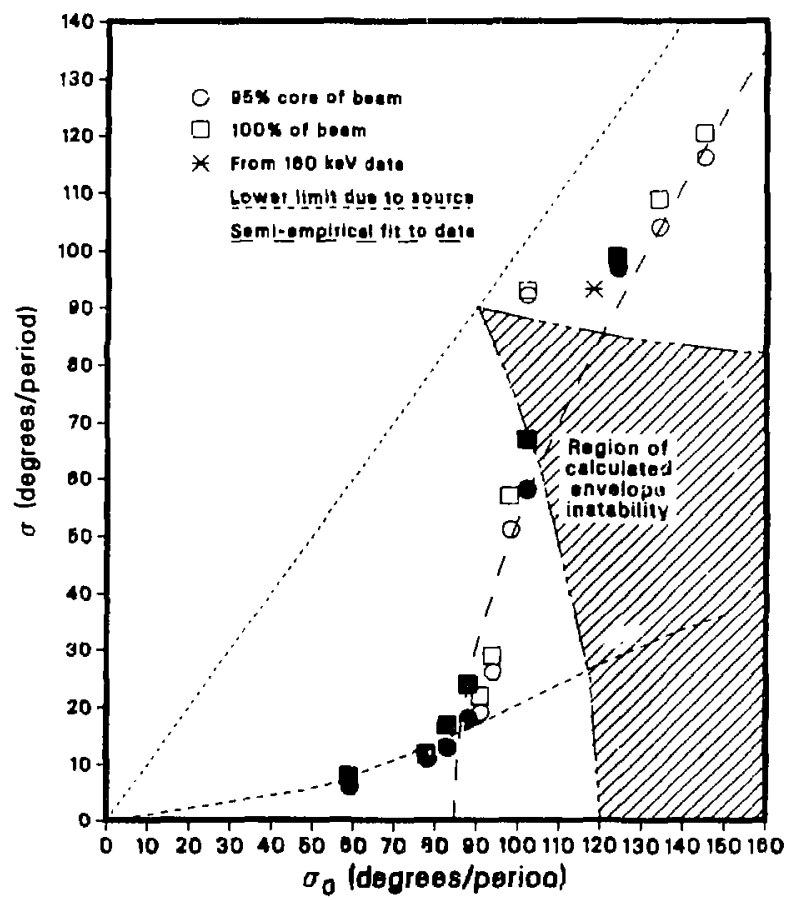

XBL $865-1840$

Figure 5.11: Summary of results for empirical stability of beams in A.G. lattice. The shaded region marks the area of instability of the envelope equations. For $\sigma_{0}<88^{\circ}$, the data are limited by the emit tance of the SBTE source. For $\sigma_{0}>88^{\circ}$, the points mark the empirical collective stability boundary. The solid symbols mark beam parameters for which the current and emittance were conserved throughout the SBTE. We have also plotted results for which the beam stabilized during transport through the channel, as discussed in the text for the various cases. The hyperbola plotted with the data is described in section 5.5 . 
By measuring the properties of the beam along the channel for various relative values of current and emittance, we determined the minimum values of $\epsilon / I$ for which $v$ 'e could maintain the beam emittance and current in the lattice. We measured the emittance at Q4, Q35, Q59, and Q80 (or Q76), spanning 38 (36) periods, and the current at Q2, Q36, Q60, and Q82, spanning 40 periods.

For $\sigma_{0}>130^{\circ}$, we were unable to transport even $3.5 \mathrm{~mA}$ through the lattice with no loss of beam, although by injecting the full $10.3 \mathrm{~mA}$ beam into the lattice we could transport as much as $7.4 \mathrm{~mA}$ to the DFC at Q82. The reason for only partial transmission for the $3.5 \mathrm{~mA}$ beam is not known, though we believe it is at least partly a result of matching and alignment problems, aggravated by the high value of $\sigma_{0}$. While for $\sigma_{0}>124^{\circ}$ we had no examples of total current and emittance conservation through the SBTE lattice, in some of the beam runs the beam had ceased its variation along the lattice by the time it reached Q35, having the same measured emittance and current for Q35 and downstream. We conclude tentatively that the beam reaches a new equilibrium by the time Q35 is reached, and that it is stable thereafter. We have included in our results data for these cases, noting that the beam is stable only from Q35 on. For the two values $\sigma_{0}=94^{\circ}$ and $\sigma_{0}=98^{\circ}$, we made measurements only during the degradation of an unstable beam, but in these instances, the beam distribution remained constant between Q59 and Q80. For these two cases, as well, we have plotted the output parameters in Fig. 5.11. More information about these beam measurements is included in section 5.3.2.

\subsubsection{Envelope measurements}

We have compared the measured RMS radius of the beam with that calculated from $\sigma_{0}$ and the measured current and emittance, using the envelope 
equations, in the last two columns of Table 5.1. The measured radius will vary along the lattice from the average value because of imperfect matching, but the resulting oscillations have been observed at low $\sigma_{0}$ to damp considerably through the channel, as shown in Fig. 5.4 for $\sigma_{0}=59^{\circ}$ and $\sigma_{0}=83^{\circ}$.

\subsubsection{Phase space measurement results}

We have calculated from solutions to the envelope equations the corresponaing values of $\sigma$ for a $\mathrm{K}-\mathrm{V}$ model beam with the same current and RMS emittance as measured for the real beam. Those values have been entered into Table 5.1 and plotted in Fig. 5.11. An analysis of these data has been published previously by Tiefenback and Keefe [25], but using values of beam energy $2 \%$ too low (caused by an error in time-of-flight measurements with a high-current beam). This calibration error resulted in quoted values for the paraxial $\sigma_{0}$ that were too high by about $1^{\circ}$ for $\sigma_{0} \sim 60^{\circ}, 2^{\circ}$ for $\sigma_{0} \sim 90^{\circ}$, and $5^{\circ}$ for $\sigma_{0} \sim 150^{\circ}$. Results quoted by Tiefenback and Keefe for the range $60^{\circ} \leq \sigma_{0} \leq 150^{\circ}$ actually span the range $59^{\circ} \leq \sigma_{0} \leq 145^{\circ}$ for paraxial particles. See section 7.1 for more information on errors in $\sigma_{0}$.

In the following figures, Figs. 5.12-5.25, we show the results of measurements made over the range $88^{\circ} \leq \sigma_{0} \leq 145^{\circ}$. The letters " $\mathrm{H}$ " and "V" in the legends for the $i$ vs. $\epsilon(i)$ plots denote measurements made in the horizontal and vertical dimensions, respectively. The contour levels in the phase space data are chosen to lie at levels containing approximately $50 \%, 60 \%$, $70 \%, 80 \%, 90 \%$, and $95 \%$ of the beam. Thus the contours do not surround a region of constant current, at least in the case of unstable beams for which the current varies along the channel. An additional complication is that the two transverse dimensions are not completely independent, as they would be in the case of negligible se'f-field for linear focusing. The 4-d nature of the phase space allows variations in the contours not expected for purely 2-d 


\begin{tabular}{|c|c|c|c|c|c|c|}
\hline$\sigma_{0}$ & $I_{\text {out }}$ & $i / I$ & $E(i)$ & $\sigma(i)$ & $2 \tilde{x}$ & $R_{0}$ \\
\hline \multirow[t]{2}{*}{$59^{\circ}$} & \multirow[t]{2}{*}{15.2} & 1.00 & 1.38 & $8 .^{\circ}$ & 15.7 & 15.3 \\
\hline & & 0.95 & 0.97 & $6 .^{\circ}$ & 15.5 & 14.9 \\
\hline \multirow[t]{2}{*}{$78^{\circ}$} & \multirow[t]{2}{*}{15.2} & 1.00 & 1.32 & $12 .^{\circ}$ & 12.3 & $12 . \overline{1}$ \\
\hline & & 0.95 & 1.08 & $11 .^{\circ}$ & 12.0 & 11.8 \\
\hline \multirow[t]{2}{*}{$8^{\circ}$} & \multirow[t]{2}{*}{15.2} & 1.00 & 1.6 & $17 .^{\circ}$ & 12.5 & 11.6 \\
\hline & & 0.95 & 1.15 & $13 .^{\circ}$ & 12.2 & 11.2 \\
\hline \multirow[t]{2}{*}{$88^{\circ}$} & \multirow{2}{*}{$\begin{array}{r}14.3 \text { of } \\
15.2 \text { inj. }\end{array}$} & 1.00 & 2.00 & $24 .^{\circ}$ & 11.6 & 11.0 \\
\hline & & 0.95 & 1.45 & $18 .^{\circ}$ & 11.2 & 10.5 \\
\hline \multirow[t]{2}{*}{$91^{\circ}$} & \multirow{2}{*}{$\begin{array}{r}13.0 \text { of } \\
15.2 \text { inj. }\end{array}$} & 1.00 & 1.54 & $22 .^{\circ}$ & 10.3 & 10.1 \\
\hline & & 0.95 & 1.26 & $19 .^{\circ}$ & 9.9 & 9.8 \\
\hline \multirow[t]{2}{*}{$95^{\circ}$} & \multirow{2}{*}{$\begin{array}{c}10.6 \text { of } \\
15.2 \text { inj. }\end{array}$} & 1.00 & 1.68 & $29 .^{\circ}$ & 9.5 & 9.1 \\
\hline & & 0.95 & 1.38 & $26 .^{\circ}$ & 8.8 & 8.8 \\
\hline \multirow[t]{2}{*}{$98^{\circ}$} & \multirow{2}{*}{$\begin{array}{c}8.5 \text { of } \\
10.3 \mathrm{inj} .\end{array}$} & 1.00 & 3.33 & $57 .^{\circ}$ & 9.4 & 9.3 \\
\hline & & 0.95 & 2.56 & $51 .{ }^{\circ}$ & $8 . \overline{9}$ & 8.6 \\
\hline \multirow[t]{2}{*}{$102^{\circ}$} & \multirow[t]{2}{*}{5.4} & 1.00 & 2.65 & $67 .^{\circ}$ & 7.2 & 7.8 \\
\hline & & 0.95 & 1.85 & $58 .^{\circ}$ & 6.5 & $6 . \overline{9}$ \\
\hline \multirow[t]{2}{*}{$102^{\circ}$} & \multirow{2}{*}{$\begin{array}{c}5.3 \text { of } \\
5.4\end{array}$} & 1.00 & 8.8 & $93 .^{\circ}$ & 12.8 & 12.5 \\
\hline & & 0.95 & 7.9 & $92 .^{\circ}$ & 12.2 & 11.9 \\
\hline \multirow{2}{*}{$\begin{array}{c}118^{\circ} \\
(160 \mathrm{keV})\end{array}$} & \multirow[t]{2}{*}{3.45} & 1.00 & 2.16 & $93 .^{\circ}$ & 6.6 & 5.9 \\
\hline & & 0.95 & $=$ & - & - & 二 \\
\hline \multirow[t]{2}{*}{$124^{\circ}$} & \multirow[t]{2}{*}{5.4} & 1.00 & 4.00 & $99 .^{\circ}$ & 8.2 & 8.6 \\
\hline & & 0.95 & 3.5 & $970^{\circ}$ & 7.6 & 8.1 \\
\hline \multirow[t]{2}{*}{$134^{\circ}$} & \multirow{2}{*}{$\begin{array}{r}2.7 \text { of } \\
3.4 \text { inj. }\end{array}$} & 1.00 & 2.06 & $108 .^{\circ}$ & 6.2 & 6.2 \\
\hline & & $0 . \overline{95}$ & 1.67 & $1039^{\circ}$ & 5.6 & 5.6 \\
\hline \multirow[t]{2}{*}{$134^{\circ}$} & \multirow{2}{*}{$\begin{array}{c}5.0 \text { of } \\
10.3 \text { inj. }\end{array}$} & 1.00 & 3.9 & $110^{\circ}$ & 8.2 & 8.5 \\
\hline & & 0.95 & 3.2 & $105 .^{\circ}$ & 7.8 & 7.8 \\
\hline \multirow[t]{2}{*}{$145^{\circ}$} & \multirow{2}{*}{$\begin{array}{l}2.5 \text { of } \\
3.4 \mathrm{inj} \text {. }\end{array}$} & 1.00 & 2.7 & $125 .^{\circ}$ & 6.9 & 7.2 \\
\hline & & $\overline{0.95}$ & 2.2 & $121 .^{\circ}$ & 6.4 & 6.5 \\
\hline \multirow[t]{2}{*}{$145^{\circ}$} & \multirow{2}{*}{$\begin{array}{c}4.6 \text { of } \\
10.3 \mathrm{inj} .\end{array}$} & 1.00 & 3.4 & $116 .^{\circ}$ & 7.8 & 8.1 \\
\hline & & 0.95 & 2.8 & $111 .^{\circ}$ & 7.2 & 7.3 \\
\hline
\end{tabular}

Table 5.1: Comparison between calculated and measured beam envelope radius, including current, emittance, and derived $\sigma$ values. Values for $\sigma$ and $\sigma_{0}$ are in degrees/period, current in $\mathrm{mA}$, emittance in $10^{-7} \pi$ meter radian, and radius values in $\mathrm{mm}$. The value $R_{0}$ calculated from the envelope equations is for the midplane between quadrupoles. 
evolution (see section 6.1). The plots all cover the same area in phase space, and are drawn to the same scale, covering about \pm 35 milliradians in angle and $\pm 20 \mathrm{~mm}$ in position. The vertical scales for the perspective views are aiso the same (with one exception in Fig. 5.23), except that no allowance has been made for the varying calibration of the fetectors (from such effects as variation of the width of the collimating slits at the varinus measurement stations). All are centered on the beam centroid, which is near the beamline axis except for very high $\sigma_{0}$, for which the lens misalignment becomes important over the length of the channel. In some of the contour plots, the position axis was lost in the figure preparation, but in most of these cases, the corresponding perspective view (from the upper right-hand corner of the contour map) is shown with the same axes, fully labeled. The perspective views are included to aid interpretation of the contour maps.

The onset of instability may be seen at $\sigma_{0}=88^{\circ}$ with the full $15 \mathrm{~mA}$ beam current of the SBTE with minimum emittance. The results of our measurements are shown in Figs. 5.12 and 5.13. There is a small beam loss through the SBTE for these parameters, about 4-5\%, although the emittance is constant. The only significant change in the phase space distribution of the beam is a rearrangement of the perturbed portions of the beam visible in the Q4 contour map in Fig. 5.13. This is the same phenomenon shown in Fig. 5.2. The beam remains unchanged between Q59 and Q80. The Q4 RMS curve in Fig. 5.12 is steeper than the curves from the other measurement positions. This is a result of the RMS calculation, which weights particles far from the phase space centroid more heavily than those near the centroid. The shape of the beam contour, and not just the area in phase space occupied by the contour, influences the RMS emittance calculation.

In Figs. 5.14 and 5.15, we show the results of injecting a $10-\mathrm{mA}$ beam 
15 mA Injected into $88^{\circ}$ Lattice Output Current: $14.3 \mathrm{~mA}$
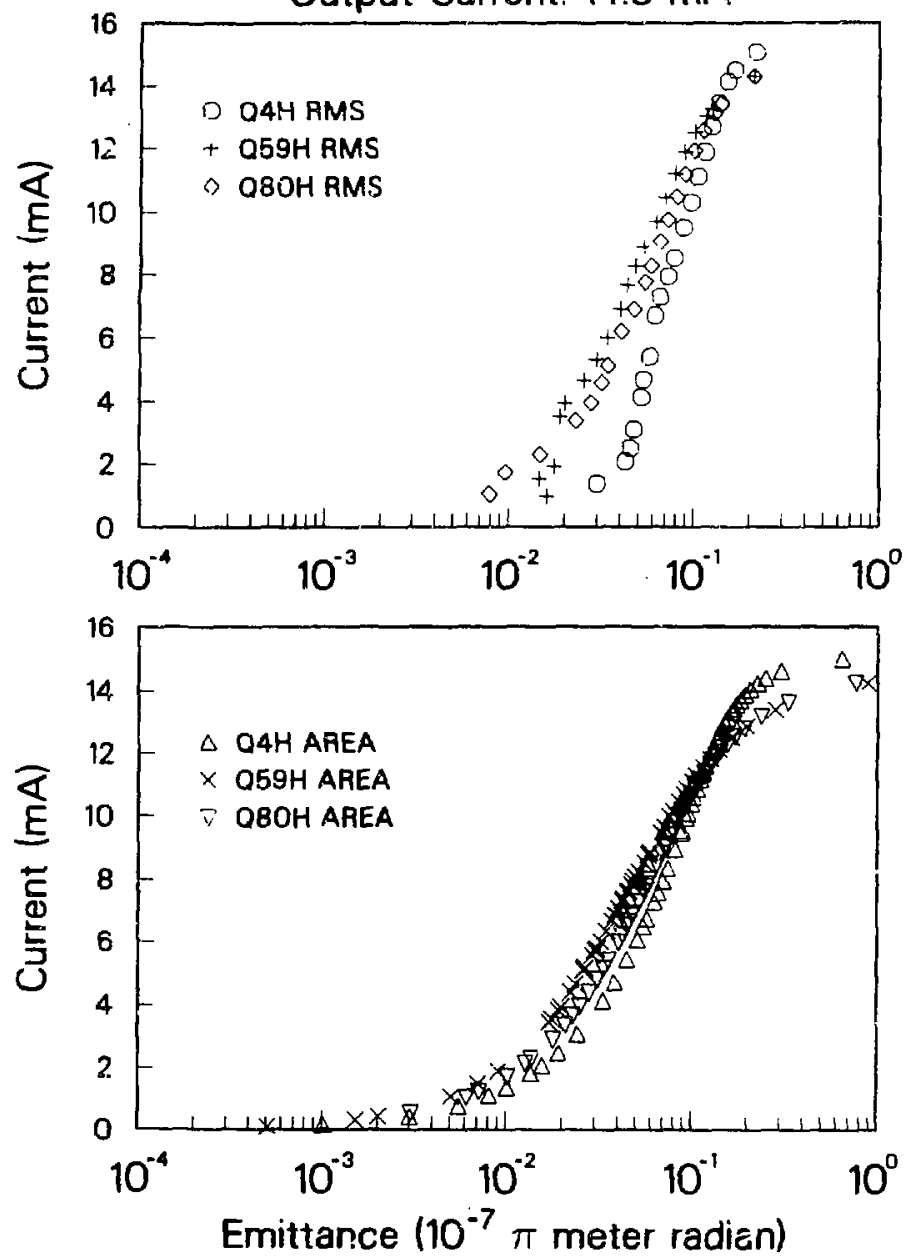

xCG $864-7168$

Figure 5.12: Current v8. emittance for $\sigma_{0}=88^{\circ}$. The Q4 RMS curve shows a higher emittance for fractional bean current because of the distortion in its phase space distribution, compared to Q59 and Q80. The distributions at Q54 and Q80, however, are very similar. 

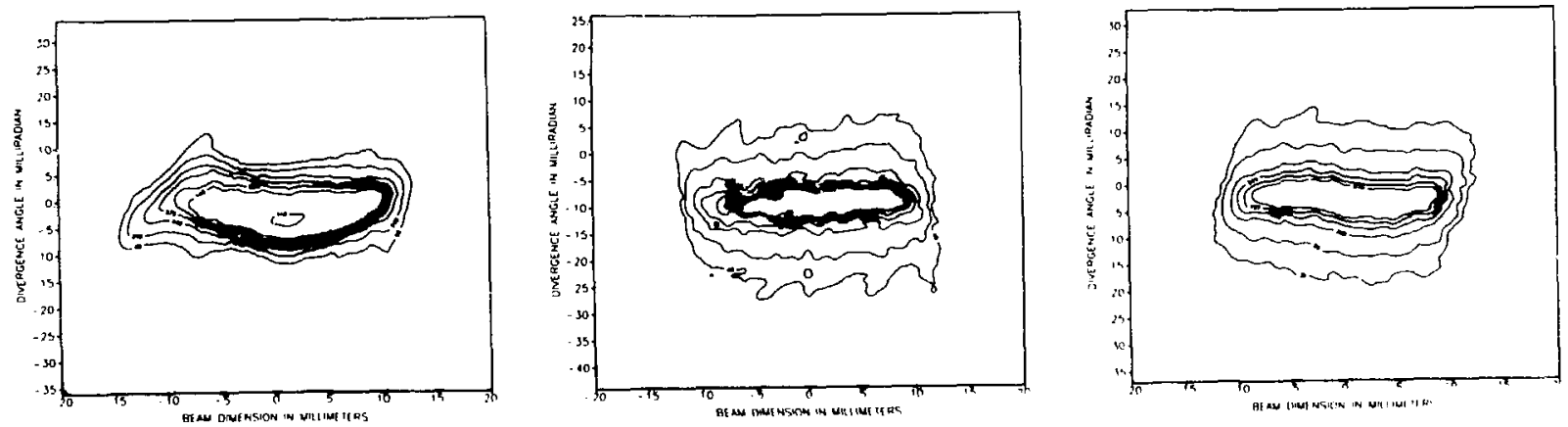

XBL 865-1873

Figure 5.13: Phase space contours for $\sigma_{0}=88^{\circ}$. The phase space distribution at Q4 appears not too severely aberrated. At a lower level than is shown in the contour map, however, there is an arm-like structure, parallel to the flat left end of the outermost contour, pointing to the upper right. This structure does not persist in measurements further along the lattice. The low-level contours (containing $90 \%$ or more of the beam), become greater in extent as the beam propagates along the channel, but the beam shows no change between Q59 and Q80. 
$10 \mathrm{~mA}$ beam injected into $\sigma_{0}=98^{\circ}$ lattice 36 Output current $8.5 \mathrm{~mA}$
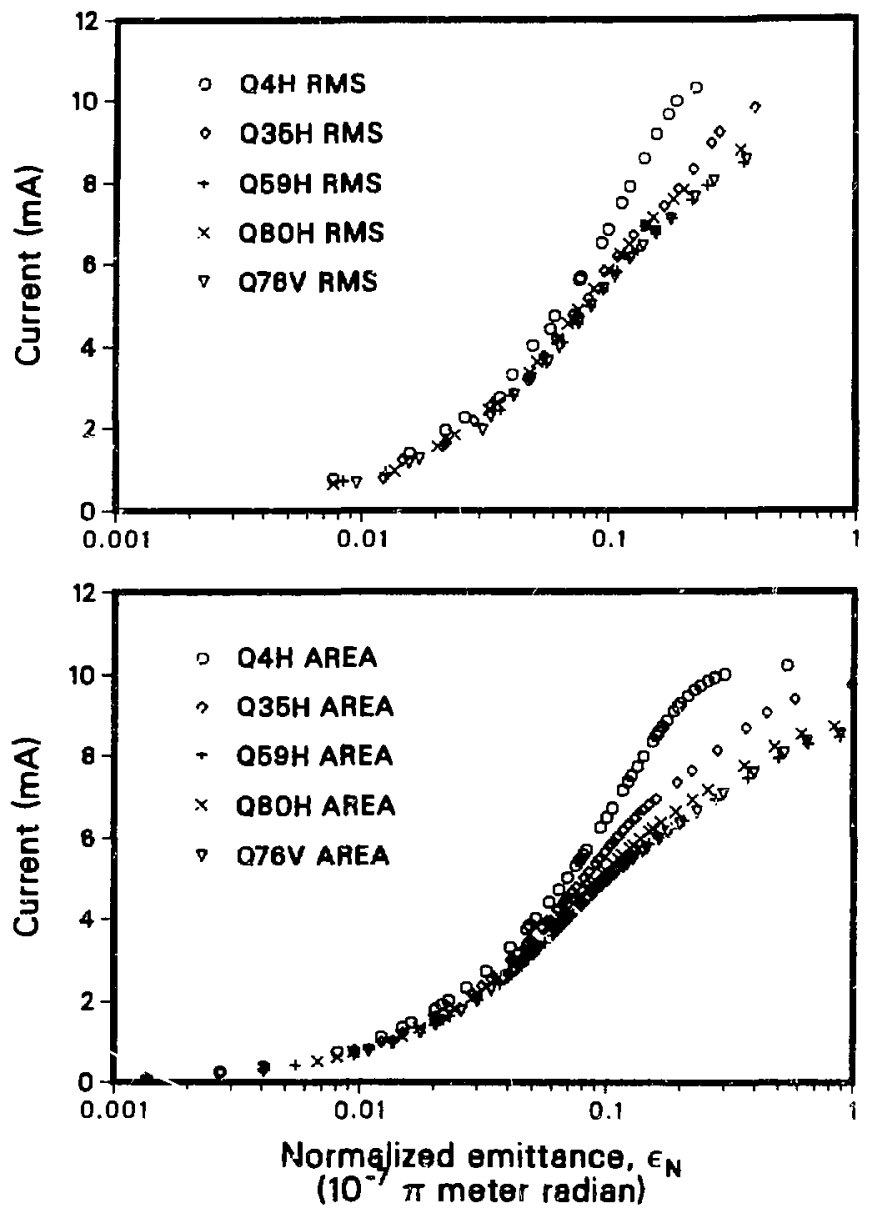

XBL 865-1891

Figure 5.14: Current vs. emittance for $\sigma_{0}=98^{\circ}$. The instability of the beam shows up in decreased current and increased emittance. The curves for Q59 and for Q76 and Q80 show that the beam is no longer changing downstream of Q59. 
(a)
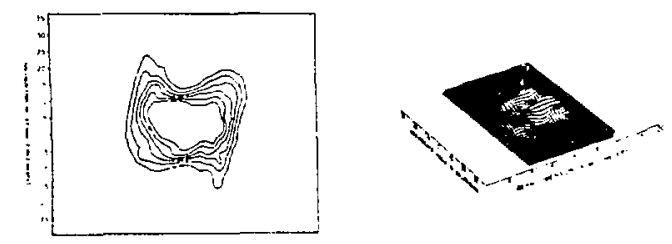

(b)
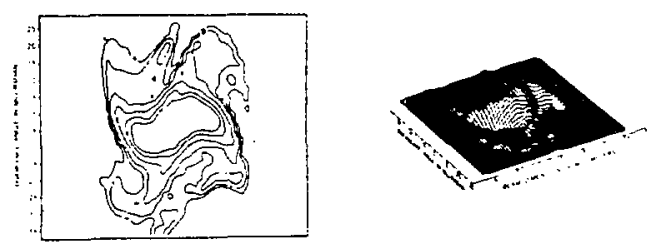

(c)
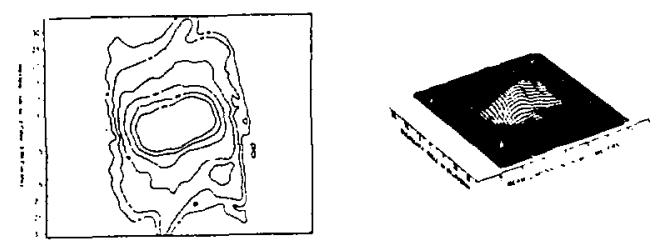

(d)
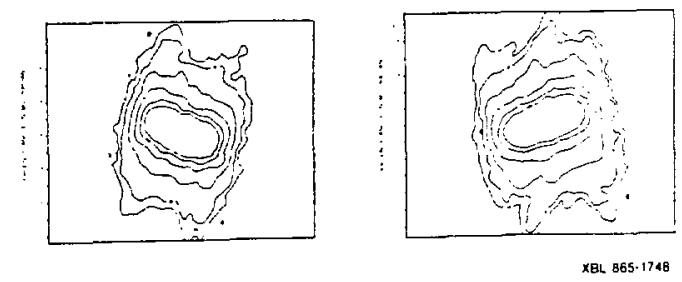

Figure 5.15: Phase space contours for $\sigma_{0}=98^{\circ}$. The perspective views are for the contour maps at the left for Q4 in row (a), Q35 in row (b), and Q59 in row (b). In row (d) are contour maps for Q80 (horizontal) on the left and Q76 (vertical) on the right. The evolution of the beam between Q4 and Q35 shows a four-pointed structure which is apparent in our other measurements only for $\sigma_{0}=102^{\circ}$. The structure is similar to that shown in some simulation work by Haber and Maschke [45] and by Hofmann [46] for strongly focused beams. A.s the beam propagates downstream, the structure becomes much less prominent, and the beam ultimately stabilizes. 
into a $\sigma_{0}=98^{\circ}$ lattice. The injector grid had been replaced by the 3 -grid eraittance control assembly, which attenuates the beam at the injector to about $10 \mathrm{~mA}$ and raises the injector emittance from about $1.4 \times 10^{-7} \pi$ meter radian to $2.0 \times 10^{-7} \pi$ meter radian. The beam is unstable even at this lower intensity, with some of the particles being pushed out in phase space and lost to the channel electrodes. The beam current stabilizes at about $8.5 \mathrm{~mA}$, with an emittance of about $2.6 \times 10^{-7} \pi$ meter radian. There is some structure visible in the phase contour plots in Fig. 5.14, similar to that shown in the simulation work of Haber, Hofmann, and others $[45,46]$, for a "fourth order" structure resonance. The beam has reached a stable configuration by the time it has reached Q59, however, showing no further change between Q59 and $Q 80$.

Without revising the matching section lens strengths, we raised $\sigma_{0}$ to $102^{\circ}$ and repeated the beam neasurements. The results are shown in Figs. 5.16 and 5.17. The contour plots are very similar to those of Fig. 5.15, although the beam does not completely come to a stable configuration before it reaches Q59, evolving somewhat more on the way to Q76. The contour map and perspective view for $\sigma_{0}=102^{\circ}$ at Q76 are very similar to those for $\sigma_{0}=$ $98^{\circ}$. We also attenuated the injector current to $5.4 \mathrm{~mA}$, and after careful adjustment of the matching section, we were able to transport the entire current through the SBTE, with an output emittance of about $(1.9 \pm 0.1) \times$ $10^{-7} \pi$ meter radian, compared to the $2.0 \times 10^{-7} \pi$ meter radian injertion emittance value. These are the parameters used for the lower set of points for $\sigma_{0}=102^{\circ}$ in Fig. 5.11.

We were unable for some time to transport the 5.4-mA beam through the lattice for $\sigma_{0}=102^{\circ}$. It is clear in Fig. 5.11 that these beam parameters place the beam very near the high-intensity (low-emittance) boundary of 


\section{$10 \mathrm{~mA}$ beem injected into $\sigma_{8}=102^{\circ}$ lattice 89 Output current $6.9 \mathrm{~mA}$}
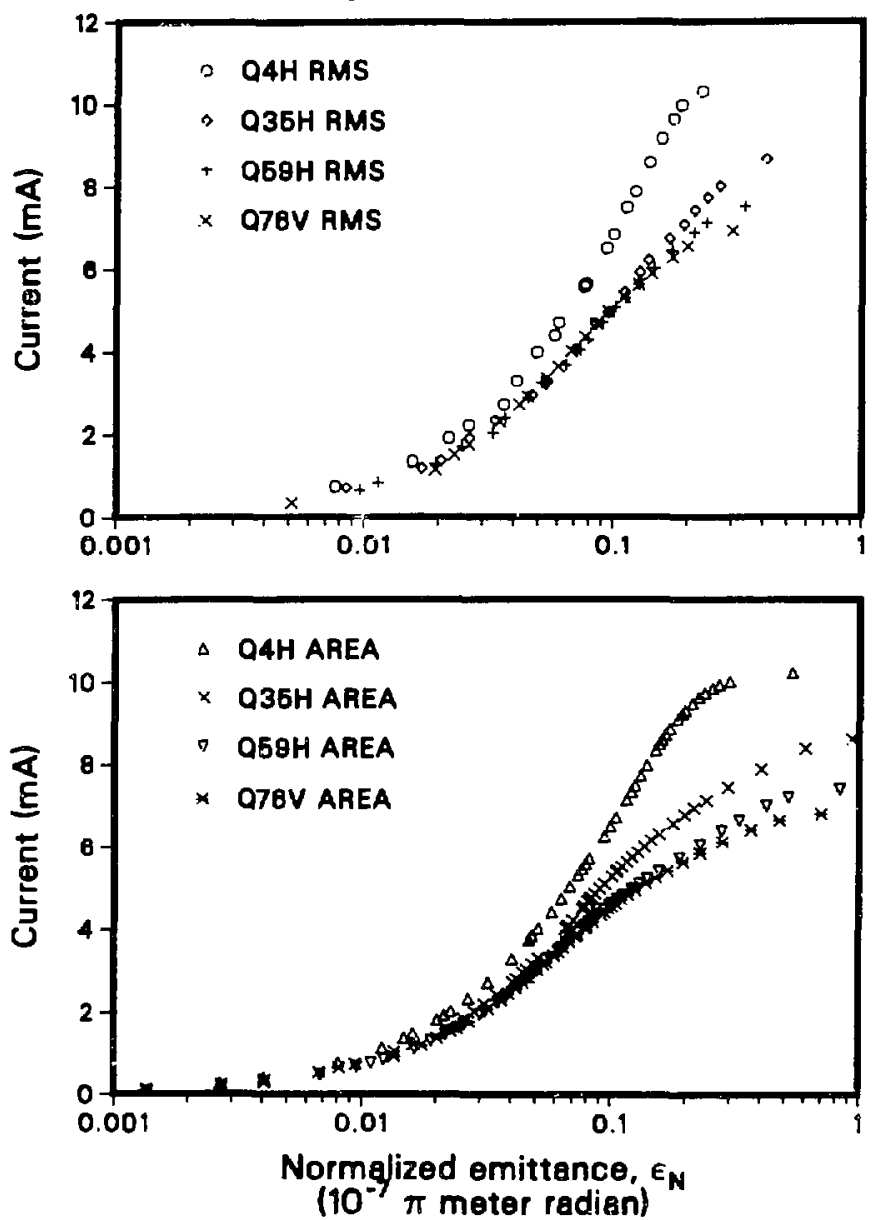

XBL $865-1890$

Figure 5.16: Current vs. emittance for $\sigma_{0}=102^{\circ}$. The major change occurs between $\mathrm{Q} 4$ and $\mathrm{Q} 35$ for the RMS curves, with the evolution nearly complete by the arrival of the beam at Q59. The current measured on the Q60 SFC is $7.2 \mathrm{~mA}$, and the current at $\mathrm{Q} 82$ is $6.9 \mathrm{~mA}$. The area emittance continues to rise even as more current is lost on the way to Q59, but little more change occurs before Q76. 

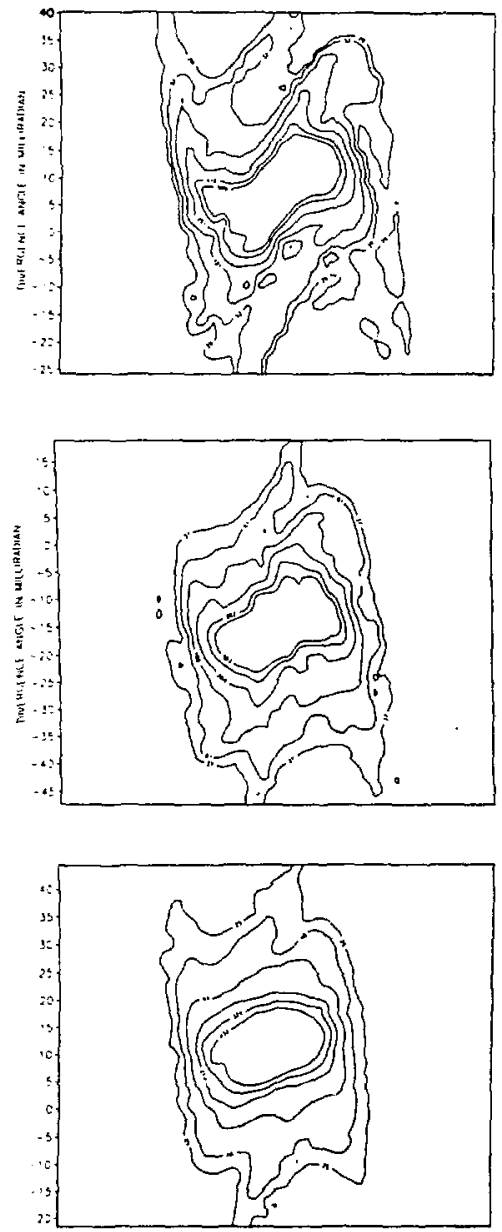

(a)

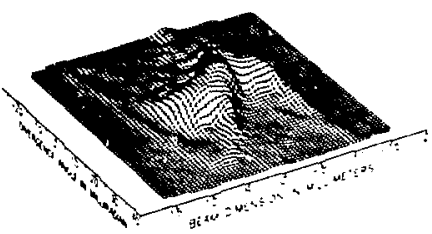

(b)

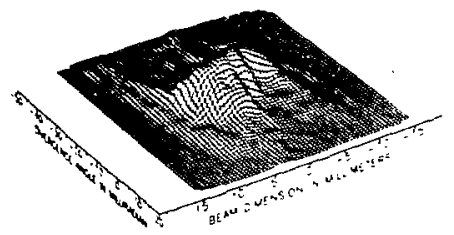

(c)

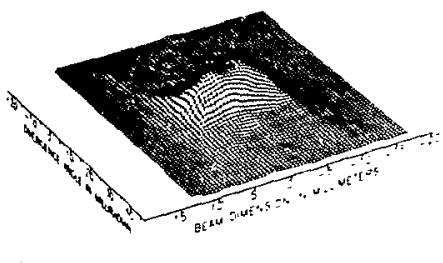

Figure 5.17: Phase space contours for $\sigma_{0}=102^{\circ}$. The perspective views correspond to the contour maps to the left of each for (a) Q35, (b) Q59, and (c) Q76. The four-pointed structure again is visible at Q35, and remains visible to some extent at Q59. The beam is very nearly stabilized by this point, evolving little in the remaining transport to $Q 76$. 
the envelope instability, and this is apparently the reason the difficulty in matching the beam into the lattice for transport to Q82 without attenuation. We also tried to use the emittance grids to raise the emittance, in an attempt to improve the current transmission, when we found that some of the beam was lost in the channel. This approach was successful for $\sigma_{0}=124^{\circ}$, as shown below, but for this case, we could obtain a maximum current of 5.3 $\mathrm{mA}$ only by raising the bias on the grids to near the breakdown iimit. We measured the beam emittance as far as Q59, obtaining values much higher than we expected at Q35 and Q59. The emittance we measured at Q4 with the emittance grid bias raised to $12 \mathrm{kV}$, was $5 \times 10^{-7} \pi$ meter radian, while the emittance measured at Q35 and Q59 was $8 \times 10^{-7} \pi$ meter radian, by far the highest value of the beam emittance that we have observed. The maximum beam size according to the envelope equations for this emittance is $20 \mathrm{~mm}$, so this emittance is close to the acceptance of the SBTE for this value of $\sigma_{0}$.

We later recognized that the source of our difficulty was the envelope instability band, and we attribute the high emittance values measured at Q35 and Q59 to the effect of the instability. Because the current was approximately conse-"red throughout the channel and because the emittance of the beam was constant for Q35 and Q59, we have included the beam parameters for this case in Table 5.1 and Fig. 5.11. We thus have observed stable transport on either side of the envelope instability band for $\sigma_{0}=102^{\circ}$. We were unable to maintain beam parameters for which tha beam was within the envelope instability band.

We made our measurements for $\sigma_{0}=118^{\circ}$ when we were operating at a particle energy of $160 \mathrm{keV}$. We used a beam current of $3.45 \mathrm{~mA}$, and raised the normalized emittance from the minimum value of $1.2 \times 10^{-7} \pi$ meter radian for that injector configuration into the neighborhood of $2.2 \times 10^{-7} \pi$ meter radian, using the emittance spoiling grids discussed in Appendix C. 
The results of this series of measurements are shown in Fig. 5.18. The error bars represent the estimated accuracy of $\pm 10 \%$, with a precision estimated irom the reproducibility and systematic consistency of the measurements to be about $\pm 5 \%$. For these measurements, the current is constant at $3.45 \mathrm{~mA}$ along the channel (equivalent to $2.25 \mathrm{~mA}$ at $120 \mathrm{keV}$ ). Sufficient clearance was provided to accomodate the increased emittance of the beam in cases of instability. For low values of injection emittance, the output emittance increases by as much a as a factor of $\mathbf{2 . 5}$, and overshoots the value ultimately found to correspond to the stability threshold. As the injection emittance is raised, the output emittance falls until the two values meet. With further increases in the injection emittance, the output emittance increases proportionally.

At the time of these measurements, we had not installed the automated data acquisition system, and data logging and analysis were manual operations. To calculate the emittance of the beam, we scanned carefully across the beam with a single traversing slit to obtain the current profile, and calculated the RMS radius of the beam. We then selected several locations within the beam for scans of the distribution in transverse angle. We always found that the RMS angular width of the distribution was nearly constant across the beam (also noted in the higher-resolution measurements made later), although the amplitude of the distribution varies with the position in real space. In this feature, the beam is similar to a Maxwellian distribution, which has a constant "temperature" throughout the beam. In contrast, the K-V model beam, with its elliptical boundary enclosing a uniform density in phase space, has a local RMS angular width decreasing with distance from the beam center. We calculated the local RMS angle of the beam near the beam centroid, and tock the emittance of the beam as four times the product of the RMS radius and angle, including the relativistic factor $\beta_{r} \gamma$ for the nor- 
๙ิ

(1)

S

त)

क

$\stackrel{2}{\underline{*}}$

ह

(4)

芌迹

足
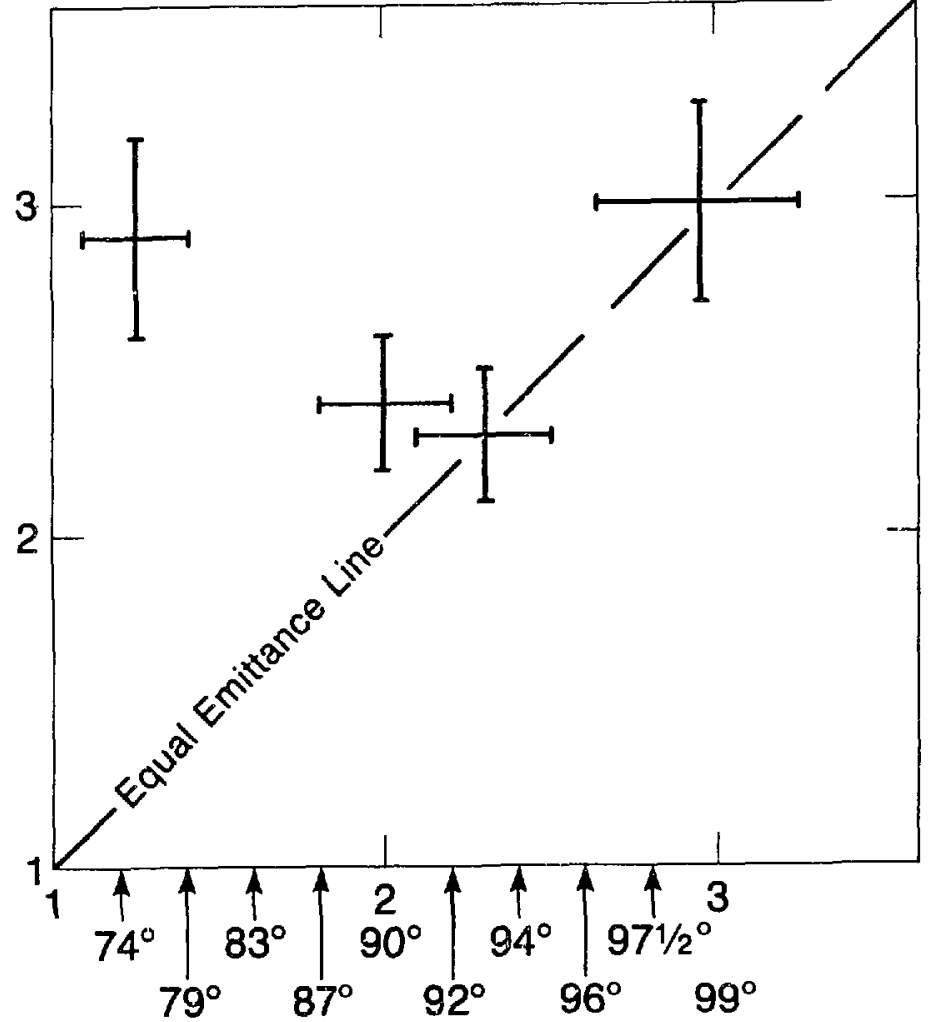

Normalized injection emittance, $\varepsilon_{\text {in }}\left(10^{-7} \pi\right.$ meter radians)

Figure 5.18: Threshold measurement for $\sigma_{0}=118^{\circ}$. For the lowest plotted initial value of the emittance, the output emittance has grown by more than a factor of 2. As the injection emittance is raised, the output emittance falls, achieving equality at about $2.3 \times 10^{-7} \pi$ meter radian. For higher values of emittance at injection, the emittance at the output rises proportionally. The error bars plotted are based on a $10 \%$ estimate of overall accuracy. 
malized emittance. This procedure neglects any aberrations in the focusing of the beam, which would be included in a full RMS calculation. Because this same procedure applied to the more-recent data obtained with the automated system gives agreement to within about $10 \%$ with the full RMS emittance calculation, we believe that our values of the emittance for the $160 \mathrm{keV}$ runs are consistent with those calculated for the $120 \mathrm{keV}$ runs, which comprise the bulk of the data :eported hore.

We made a similar threshold measurement for $\sigma_{0}=124^{\circ}$, as shown in Fig. 5.19. The injecied beam current was $5.4 \mathrm{~mA}$, at an energy of $120 \mathrm{keV}$. The resulting beam has a smaller clearance from the quadrupoles than was available for the $160 \mathrm{keV}$ measurement. For low values of injection errittance, the collective dagradation of the beam resulted in approxizaately a $10 \%$ beam loss, but the beam current became constant along the lattice for $\epsilon_{95} \sim 3.5 \times 10^{-7} \pi$ meter radian. For this reason, we have plotted both ine beam emittance and the ratio of emittance to current $\left(\varepsilon_{95} / 0.95 I\right)$ at the downstream end of the SBTE against the corresponding parameters at injection. The total beam current of $5.4 \mathrm{~mA}$ was transported to the end of the channel at the crossover point, for which the injection and output emitiances were equal. As the injection emittance was raised further, the output curient fell again slightly, although the output emittance still exceeded the injection value.

In Figs. 5.20 and 5.21, we show the results of the measurements at the crossover point, for which the bearn current was $5.4 \mathrm{~mA}$ and the normalized emittance was $3.5 \times 10^{-7} \pi$ meter radian. The initial measurement of the phase space contours ai Q4 show a great distortion of the phase space structure of the beam, with extensive gaps (relative to the peak density) in the distribution. These fili in as, presumably, the two transvarse dimensions couple together to hide the voids in 4-dimensional phase space from detection 


\section{Variation of $\epsilon$ and $\epsilon / /$ at channel exit with values at injection}
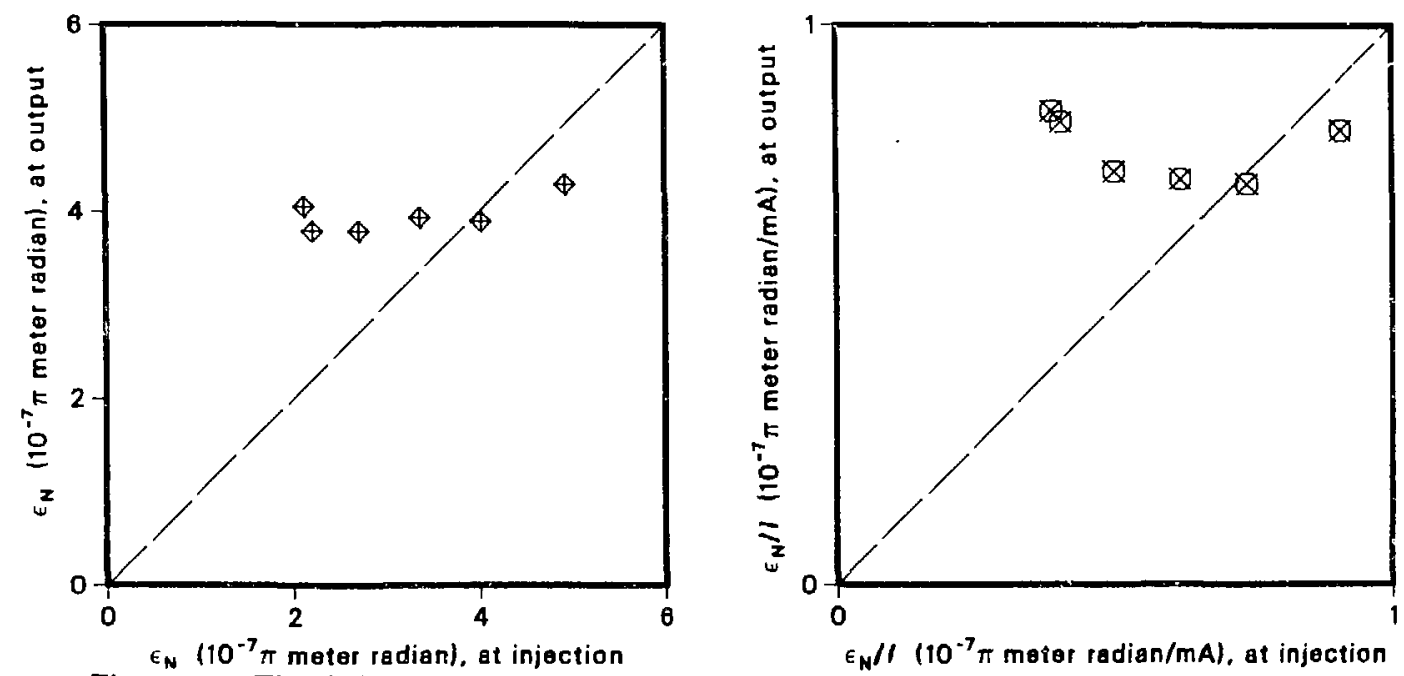

Figure 5.19: Threshold measurement for $\sigma_{0}=124^{\circ}$, showing behavior similar to that for $\sigma_{0}=118^{\circ}$. Emittance growth for unstable injection is accompanied by a small beam loss for the larger-diameter beam used here. The beam loss diminishes as the input emittance is increased, with the output emittance remaining nearly constant until the injection emittance rose above about $3.5 \times 10^{-7} \pi$ meter radian. The plot of $\epsilon_{N} / I$ at the end of the SBTE vs. t! e correspording value at injection shows the sime general features as for $\sigma_{0}=118^{\circ}$. The total beam current is about $5.4 \mathrm{~mA}$. 
$5.4 \mathrm{~mA}$ beam injected into $\sigma_{0}=124^{\circ}$ lattice 96
Output current $5.4 \mathrm{~mA}$
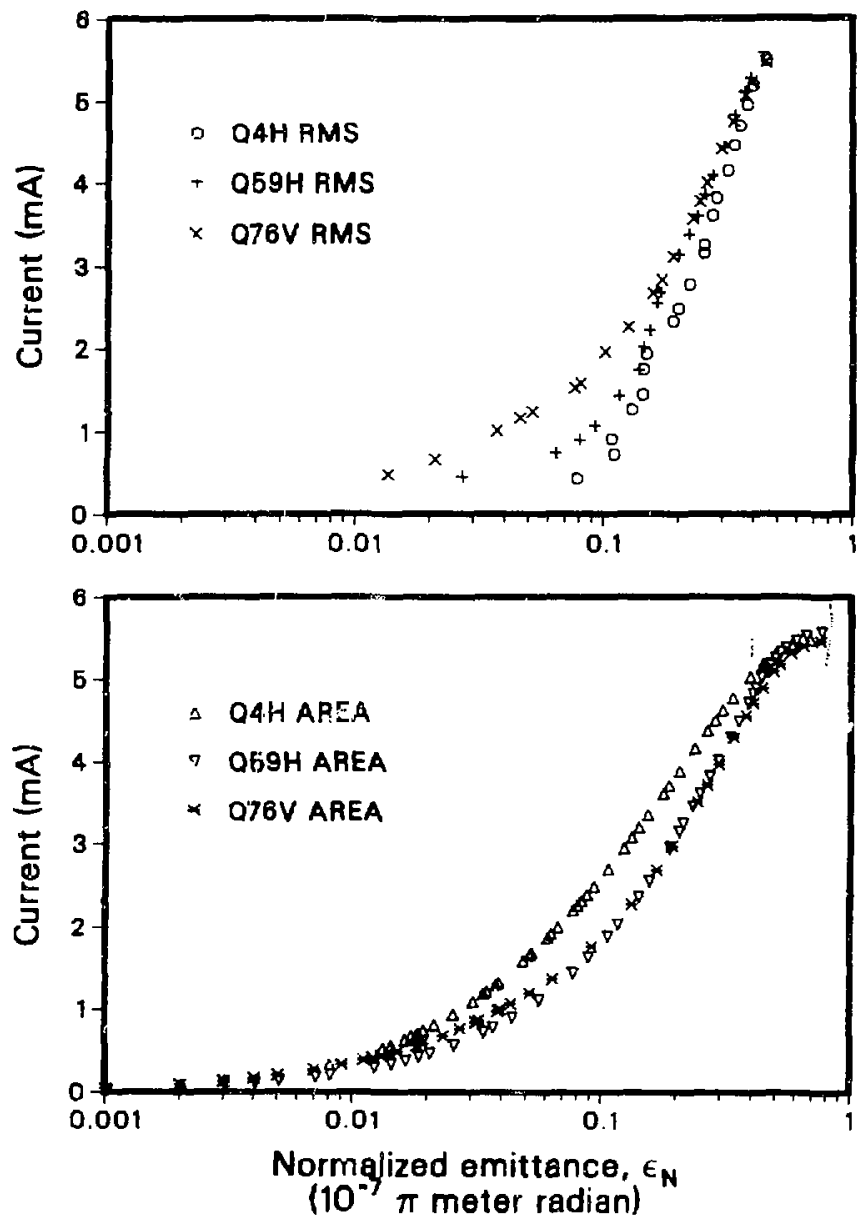

XBL 865-1889

Figure 5.20: Current vs. emittance for $\sigma_{0}=124^{\circ}$. The behavior is similar to that of the previous examples, but the total emittance and current are constant throughout the lattice. The RMS emittance for the higher level partial beam distribution decreases, while for the area curves, the partial emittance increases between $Q 4$ and $Q 59$, becoming constant thereafter. The season is clear from Fig. 5.21. 

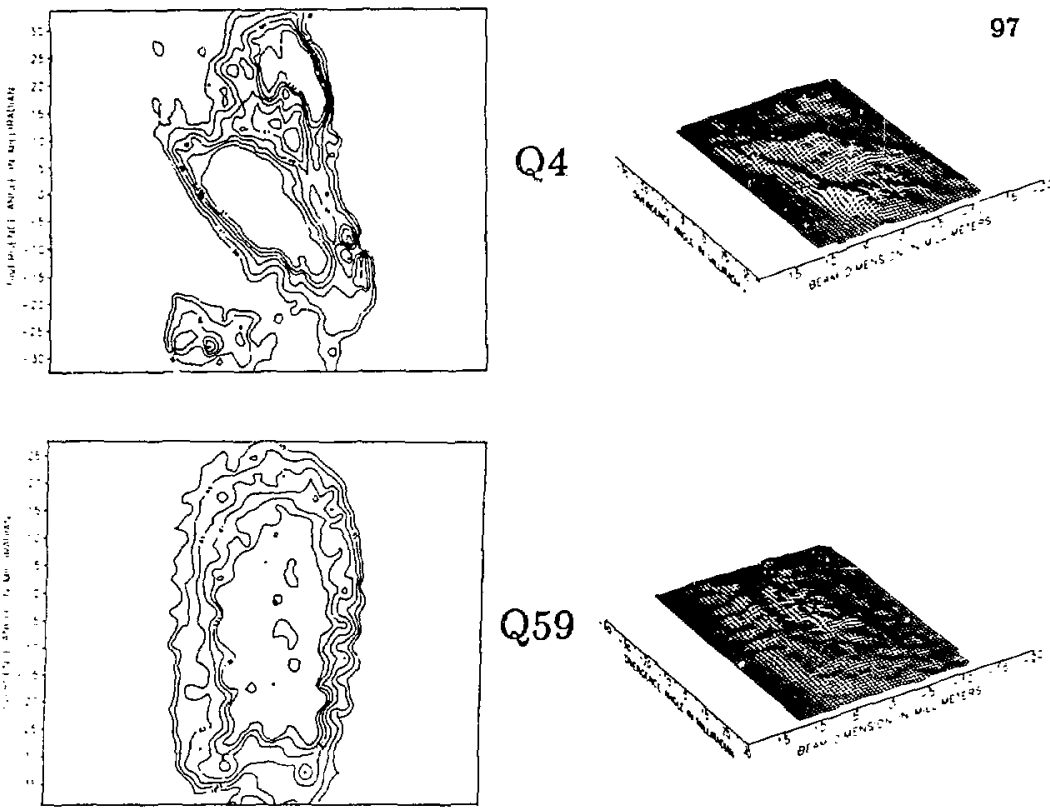

Q59
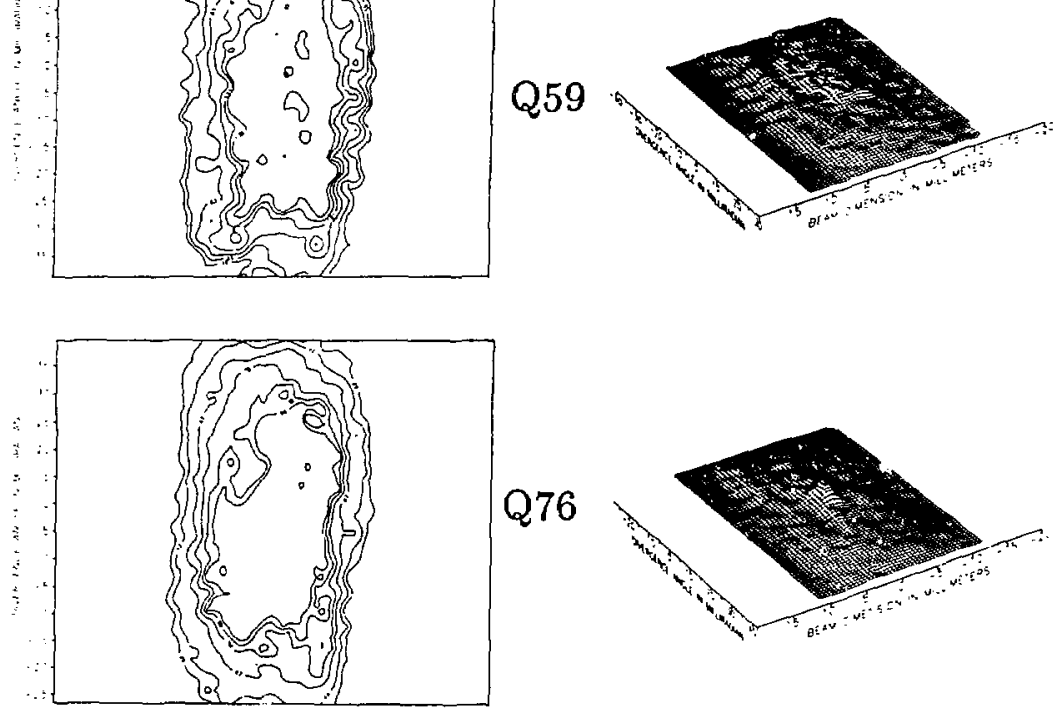

XEL $865-1740$

Figure 5.21: Phase space contours for $\sigma_{0}=124^{\circ}$. The data are shown in rows for (top to bottom) Q4, Q59, and Q76. The distribution at Q4 is greatly altered by the emittance grids at the high bias $(10 \mathrm{kV})$ in use. This structure was not present for the weaker bias potentials generally used, and it is not present in the downstream data. The dissipation of the structure introduced by the grids is the reason for the great increase in the partial area emittance curves shown in Fig. 5.20. 
by a two-slit measurement. The total area of the distribution does not increase; although the beam particles distribute themselves more evenly in the two-dimensional phase space. The RMS-derived emittance is also constant. The potential on the emittance grids for this measurement was $10 \mathrm{kV}(\sim 8 \%$ of the particle energy). For bias values below about $6 \mathrm{kV}$, the transverse phase space of the beari does not show the structure apparent for the $Q 4$ measurement of Fig. 5.21 . For the $160 \mathrm{keV}, \sigma_{0}=118^{\circ}$, beam measurements, the grid potential was about $6 \mathrm{kV}(\sim 4 \%$ of the particle energy).

For our measurements for $\sigma_{0}=134^{\circ}$ and $\sigma_{0}=145^{\circ}$, we could not transport $100 \%$ of the injected beam to Q82, even for currents as low as $3.4 \mathrm{~mA}$. We attribute this at least in part to lens misalignments, which becorne marleedly more important at high values of $\sigma_{0}$. We show in Figs. 5.22 and 5.23 a series of measurements for $\sigma_{0}=134^{\circ}$, with $2.4 \mathrm{~mA}$ at injection and $2.7 \mathrm{~mA}$ of current at the output of SBTE and the emittance grids left grounded. In Fig. 5.23, the Q7E data show the most severe pulse-to-pulse variations we experienced in the course of the experiment. The experiment was well-behaved for the first half of the data acquisition period, giving poorer reproducibility over only the last portion the data for this rua. There are only a few points having spurious high currents outside of the main body of the beam, and a few spurious zeroes inside the beam distribution. The overall outline of the beam in phase space at Q76 is very similar to the results for Q35 and $\mathrm{Q} 59$, and the emittances are also very close. The value we calculate for the emittance of the beam at Q76 is not sensitive to the positions of the spurious data, but is dominated by the well-behaved points comprising the body of the distribution, and we believe that the derived value of the emittance for Q76 is accurate. The emittance measured at Q35 was about $1.75 \times 10^{-7} \pi$ meter radian, which is less than the source emittance value of $2.0 \times 10^{-7} \pi$ meter 
$3.4 \mathrm{~mA}$ beam injacted into $\sigma_{\mathrm{p}}=134^{\circ}$ lattice Output current $2.7 \mathrm{~mA}$
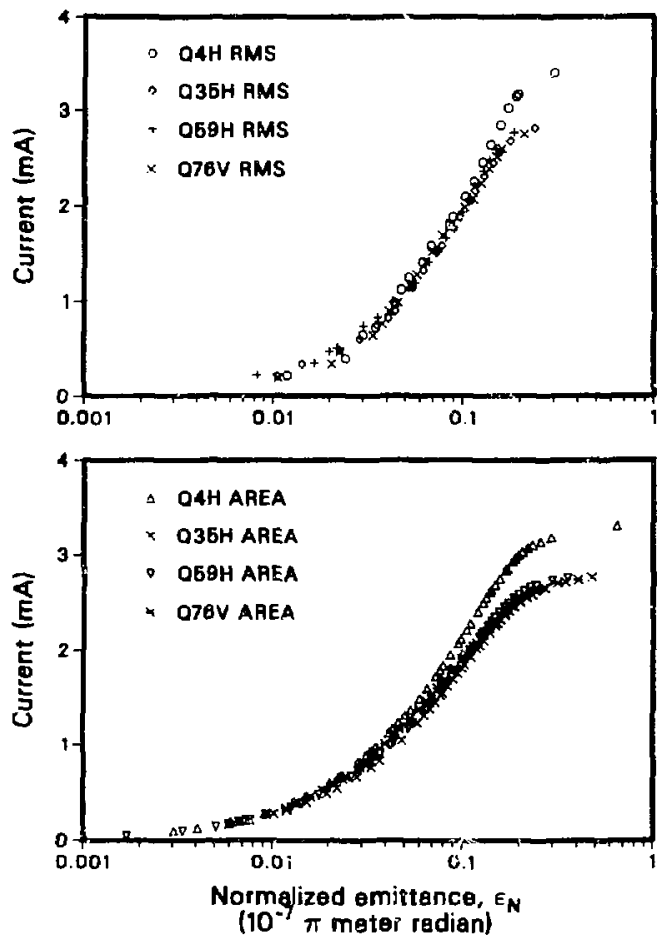

XBL. B65-1888

Figure 5.22: Current vs. emittance for $\sigma_{0}=134^{\circ}$. The exact current in the beam for Q35 and Q59 is not certain, because of the absence of Faraday cup measurements. The current used for these plots is calculated by summing the data from the phase space measurements, using the cup-measured output current of $2.7 \mathrm{~mA}$ for the measurements at Q59 and Q76, for which the sum of the phase space points indicates, respectively, 2.6 and $2.2 \mathrm{~mA}$ of current. The distribution is apparently constant downstream of Q35, apart from the possible loss of a small amount of beam. The emittance drops somewhat from Q4 to Q35, but is constant through the remainder of the lattice. 

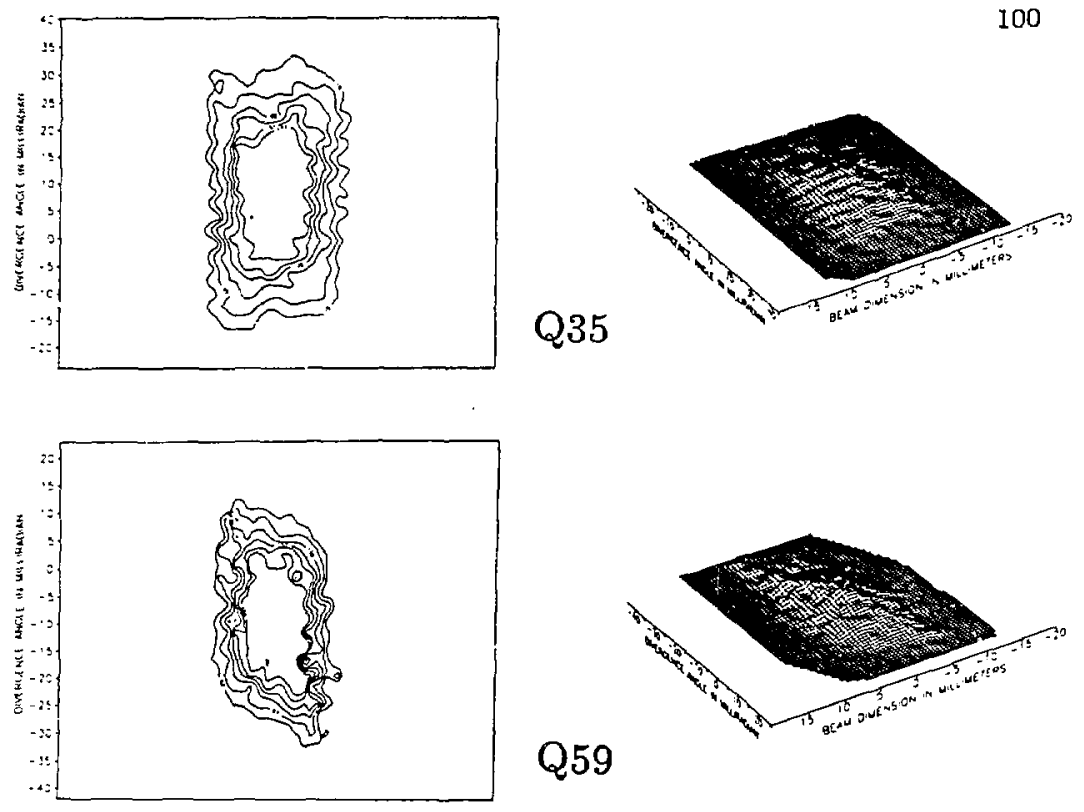

Q59
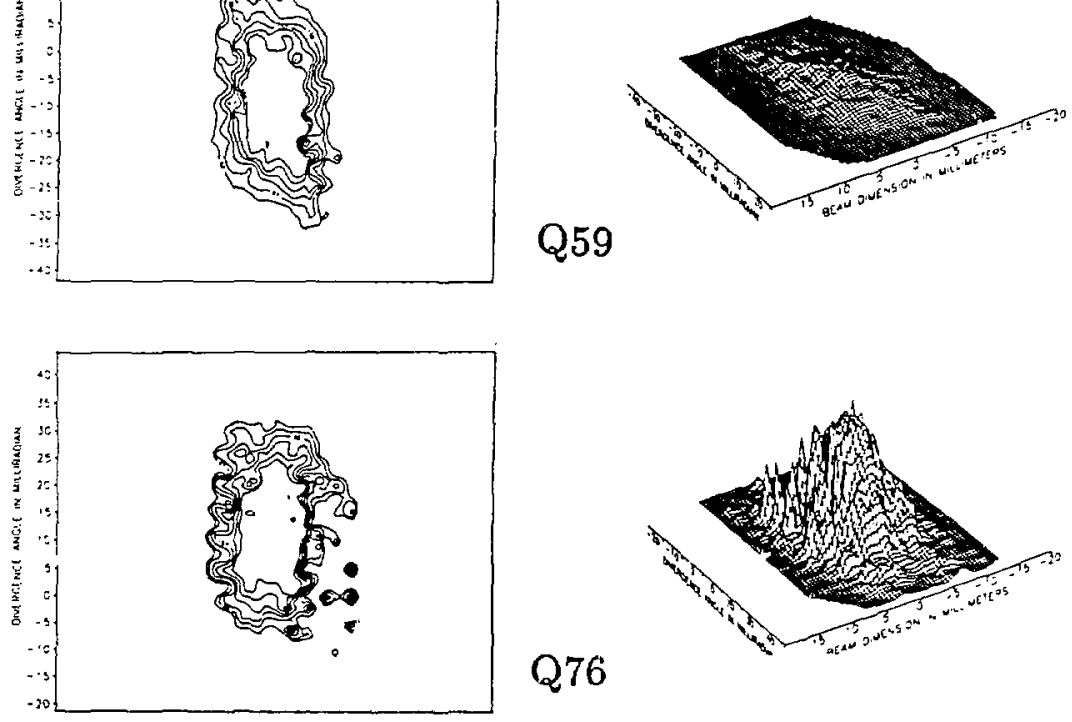

Figure 5.23: Phase space contours for $\sigma_{0}=134^{\circ}$. The data shown are, by row, for Q35, Q59, and Q76. The appearance of the Q35 and Q59 plots is similar, with no sign of a structured instability. The plots for Q76 show the effect of pulse-to-pulse variation of the beam, noticeable only for the rightmost portion of the beam, as displayed in the contour plot. See the text for more information. The emittance calculated for the beam is insensitive to the few spurious points, and the constancy of the beam distribution downstream from Q35 is evidenced by the data. 
radian. This is consistent with loss of beam due to clearance limitations. The values of emittance measured at Q59 and Q76 were, respectively, $1.6 \times 10^{-7} \pi$ meter radian and $1.7 \times 10^{-7} \pi$ meter radian.

In addition, we injected the full 10-mA beam current into the SBTE for $\sigma_{0}=134^{\circ}$, using the emittance grids set to $8 \mathrm{kV}$ bias $\left(\epsilon \simeq 3.4 \times 10^{-7} \pi\right.$ meter radian), and we were able to tune the matching section to deliver $5 \mathrm{~mA}$ of current to the Faraday cup at Q82, with an emittance of $3.2 \times 10^{-7} \pi$ meter radian. The overall ratio of emittance to current for this output beam is almost identical to that for the 2,7-ma output case discussed above.

Just as for $\sigma_{0}=134^{\circ}$, we injected the full beam current of $10 \mathrm{~mA}$ into the SBTE with $\sigma_{0}=145^{\circ}$, but using the emittance grid bias set to $7 \mathrm{kV}$ to raise the injection emittance to about $3.2 \times 10^{-7} \pi$ meter radian, and we obtained $4.6 \mathrm{~mA}$ at $\mathrm{Q} 82$, with an emittance measured at $\mathrm{Q} 76$ of $2.8 \times 10^{-7} \pi$ meter radian. The data are shown in Figs. 5.24 and 5.25. From Fig. 5.24 we see that the beam has not reached a completely stable configuration by the time it passes Q59.

For the same 3.4-mA injection curreri used for the $\sigma_{0}=134^{\circ}$ case discussed above, but with $\sigma_{0}=145^{\circ}$, we measured the current at the Q82 cup to be $2.5 \mathrm{~mA}$, with an emittance at $\mathrm{Q} 76$ of $2.2 \times 10^{-7} \pi$ meter radian. The ratio of emittance to current for the $4.6-\mathrm{mA}$ beam is about $35 \%$ below that for the 2.5-mA output case. In this parameter regime, however, the difference in the derived values for $\sigma$ is only about $10 \%$. The two output beams are compared in Fig. 5.26.

\subsection{Discussion for $\sigma_{0} \sim 90^{\circ}$}

The empirical instability region begins at the same lattice strength for which the envelope equations can become unstable, that is, $\sigma_{0} \simeq 90^{\circ}$. However, detailed comparison of the data with envelope instability predictions 
$10 \mathrm{~mA}$ beem injected into $\sigma_{0}=145^{\circ}$ lattice

Output current $4.6 \mathrm{~mA}$
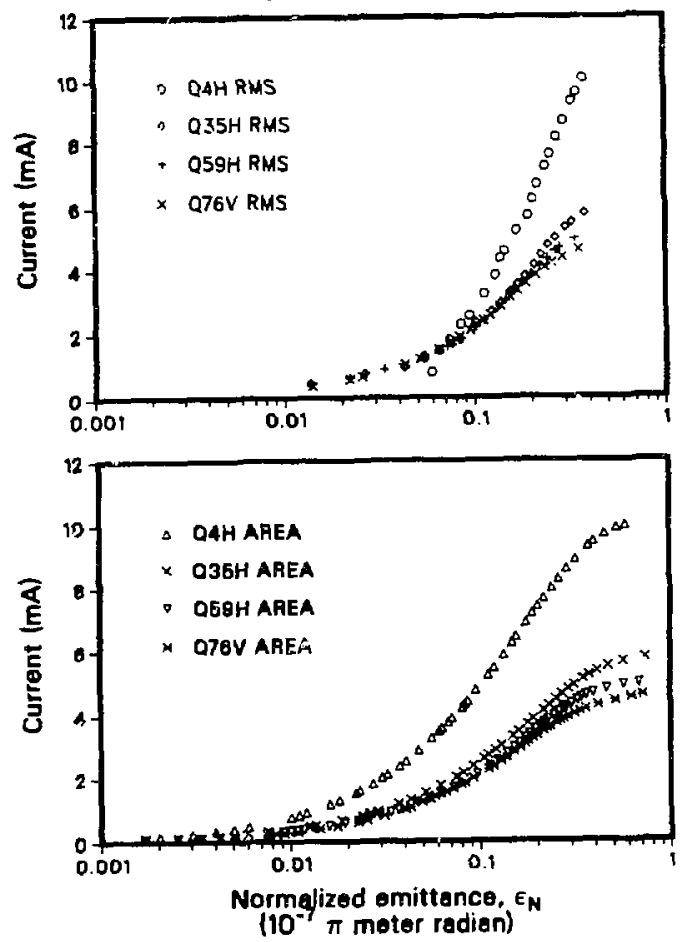

XBL B65-1887

Figure 5.24: Current vs. emittance for $\sigma_{0}=145^{\circ}$. The beam current at injection is $10 \mathrm{~mA}$, and the emittance has been raised to about $3 \times 10^{-7} \pi$ meter radian by means of the emittance grids. There is a large change in the beam distribution between Q4 and Q35, with more gradual changes in the beam occurring along the lattice. The beam does not quite reach stability before it exits the experimental channel, although the rate of change of the distribution is low. 

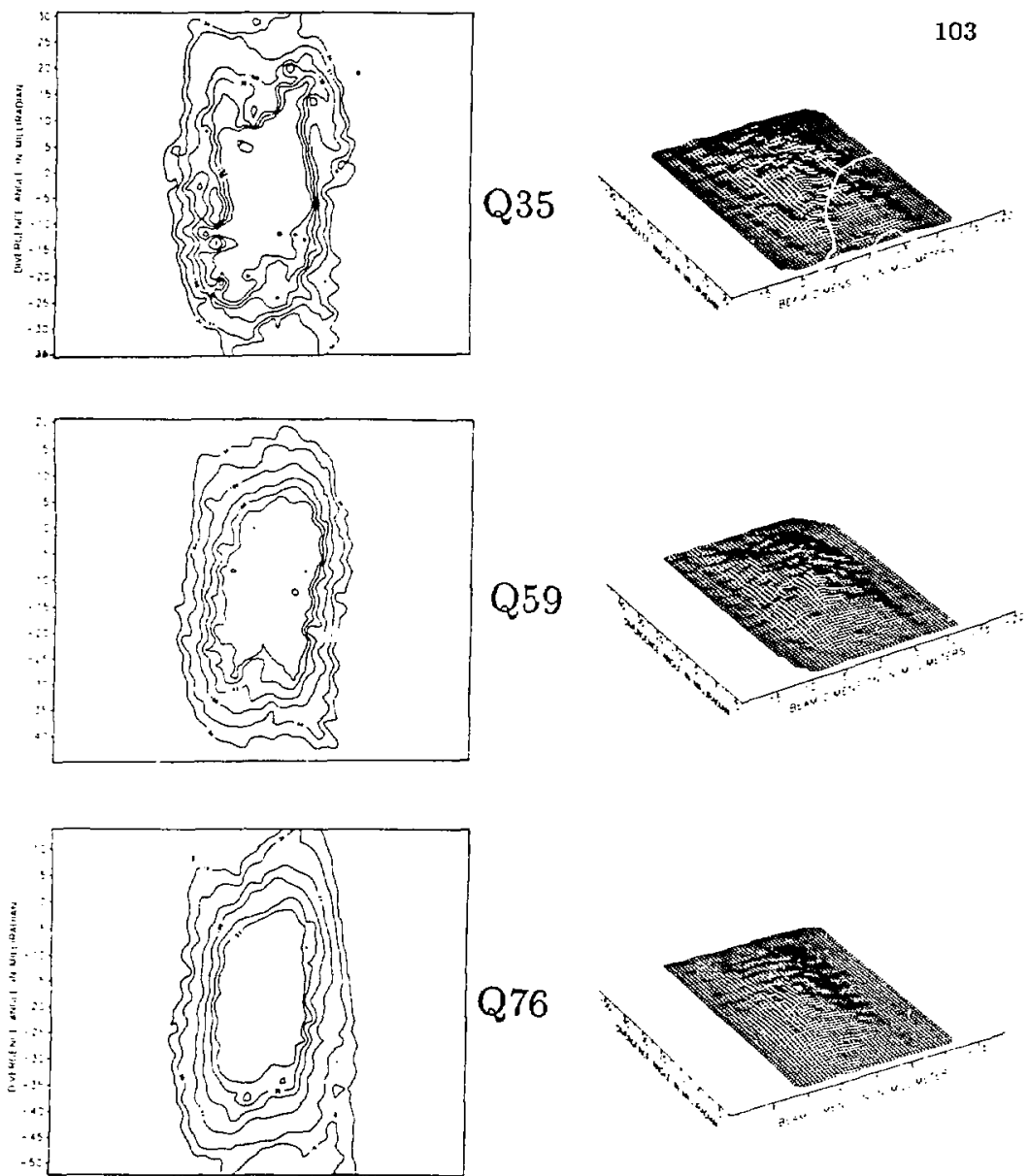

XBL $865-1744$

Figure 5.25: Phase space contours for $\sigma_{0}=145^{\circ}$. These plots are, from top to bottom, for measurements at Q35, Q59, and Q76. The Q35 plots show some structure, similar to, but not as prominent as, that in Fig. 5.21. The grid bias used to spoil the emittance at injection for this sequence of data is $7 \mathrm{kV}$. 


\section{Comparison Between $10 \mathrm{~mA}$ and $3.4 \mathrm{~mA}$ Injection into $145^{\circ}$ Lattice}

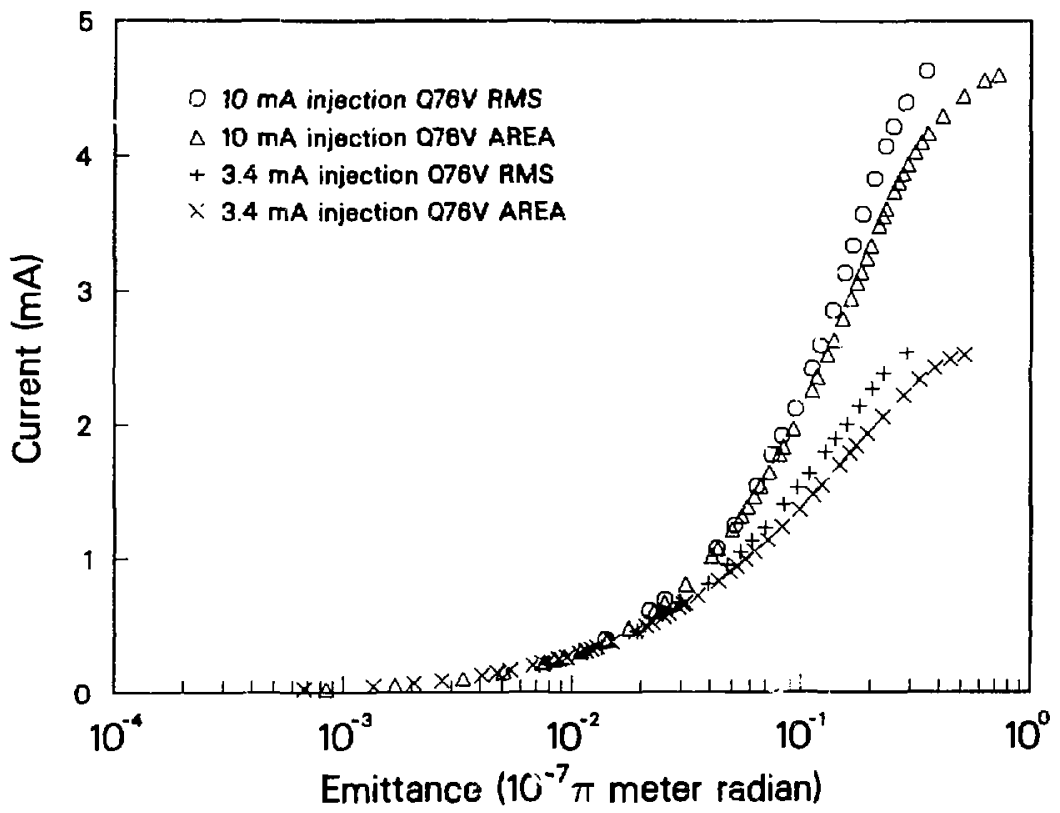

XCG 864-7164

Figure 5.26: Comparison between output beams for $\sigma_{0}=145^{\circ}$ for $10 \mathrm{~mA}$ and $3.4 \mathrm{~mA}$ injection. The two cases are somewhat different, but the higher-current beam has been generated through degradation of a 10-mA injected beam, and has not fully stabilized. The emittance and current have been changing between Q59 and Q76, and the final relative values of emittance and current may be very ciose. The figure is shown as an example of obtaining much higher current at $\mathbf{Q 8 2}$ by injecting an unstable beam, than could be provided by injecting a lower-current, more stable beam. 
shows several points of difference:

- The beam shows unstable behavior for parameters for which the "infinitely long" beam, far from the beam ends, is far from the calculated region of envelope instability, with a value of $\sigma$ too low for instability by factors of $2-4$.

- While the calculated growth rates of the envelope instability are small until $\sigma_{0}$ is raised above $100^{\circ}$, the beam shows rapid emittance degradation for $\sigma_{0}$ near $90^{\circ}$.

- The beam stabilizes at an emittance for which the beam remains on the high-intensity side of the instability band.

The maximum growth rates for the envelope instability as a function of $\sigma_{0}$, calculated using the techniques of Laslett [16], by perturbation of the envelope equations, are shown in Fig. 5.27. This calculation was for hard-edge quadrupoles with an occupancy factor of 0.5 , but the results are insensitive to the quadrupole occupancy factor. Comparison with calculations both for a thin lens lattice and for the SBTE focusing field representation given in Appendix $\mathrm{D}$ shows a difference of only about $1^{\circ}$ in $\sigma$ for the instability thresholds, and nearly identical peak growth rates. Simulation studies have been done by Haber [47] for a low-emittance beam, passing through a lattice set to $\sigma_{0}=90^{\circ}$. As the current was slowly ramped up (and $\sigma$ dropped), the emittance increased only after the beam passed a threshold intensity corresponding to $\sigma \simeq 30^{\circ}$. Our experimental value for the limiting $\sigma$ for $\sigma_{0}=90^{\circ}$ is about $\mathbf{2 5}^{\circ}$, as shown in Fig. 5.11.

The evolution of unstable beams as shown in the $i$ vs. $\epsilon(i)$ plots displays some interesting behavior. For $\sigma_{0}=94^{\circ}$, the beam degrades significantly in only 15 periods, as shown in Figs. 5.28 and 5.29, although the growth 
Maximum Growth Rates for the Envelope Instability for $1 / 2$ Occupancy Hard-edge Quadrupoles

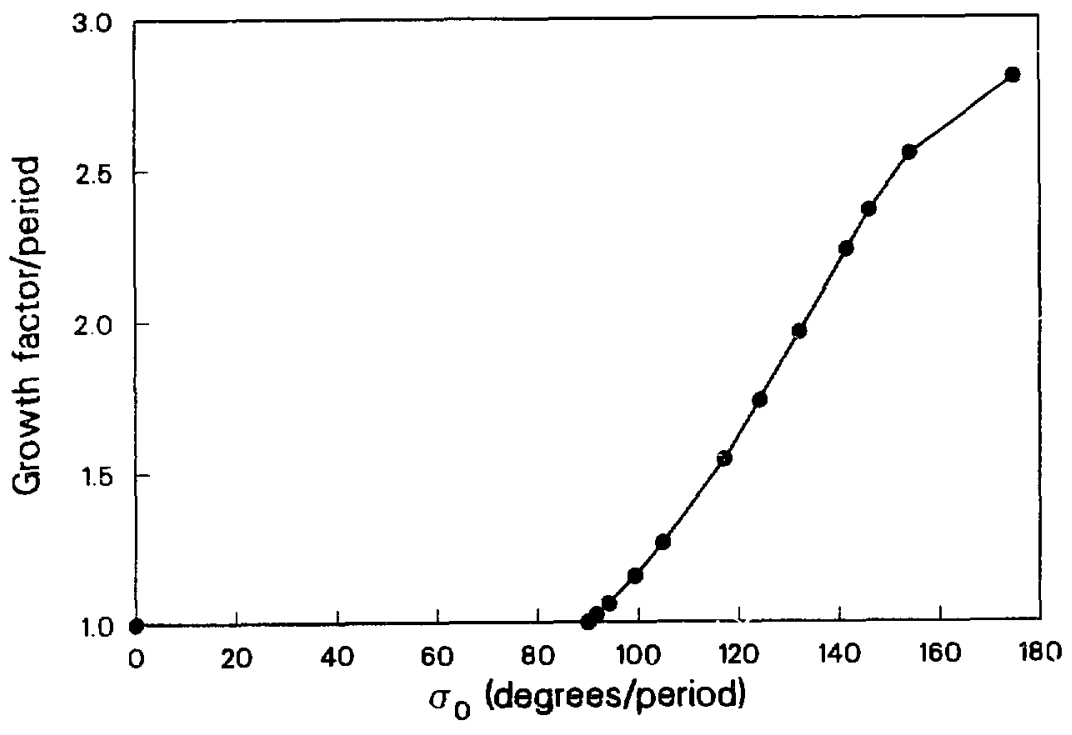

XCG $864-7152$

Figure 5.27: Maximum growth rates for the envelope instability for hard-edge quadrupoles with an occupancy factor of 0.5 . The thresholds and growth rates were compared with both thin lens quadrupoles and the SBTE model given in Appendix D, and gave agreement within about $1^{\circ}$ in thresholds and a few percent in growth rate. 

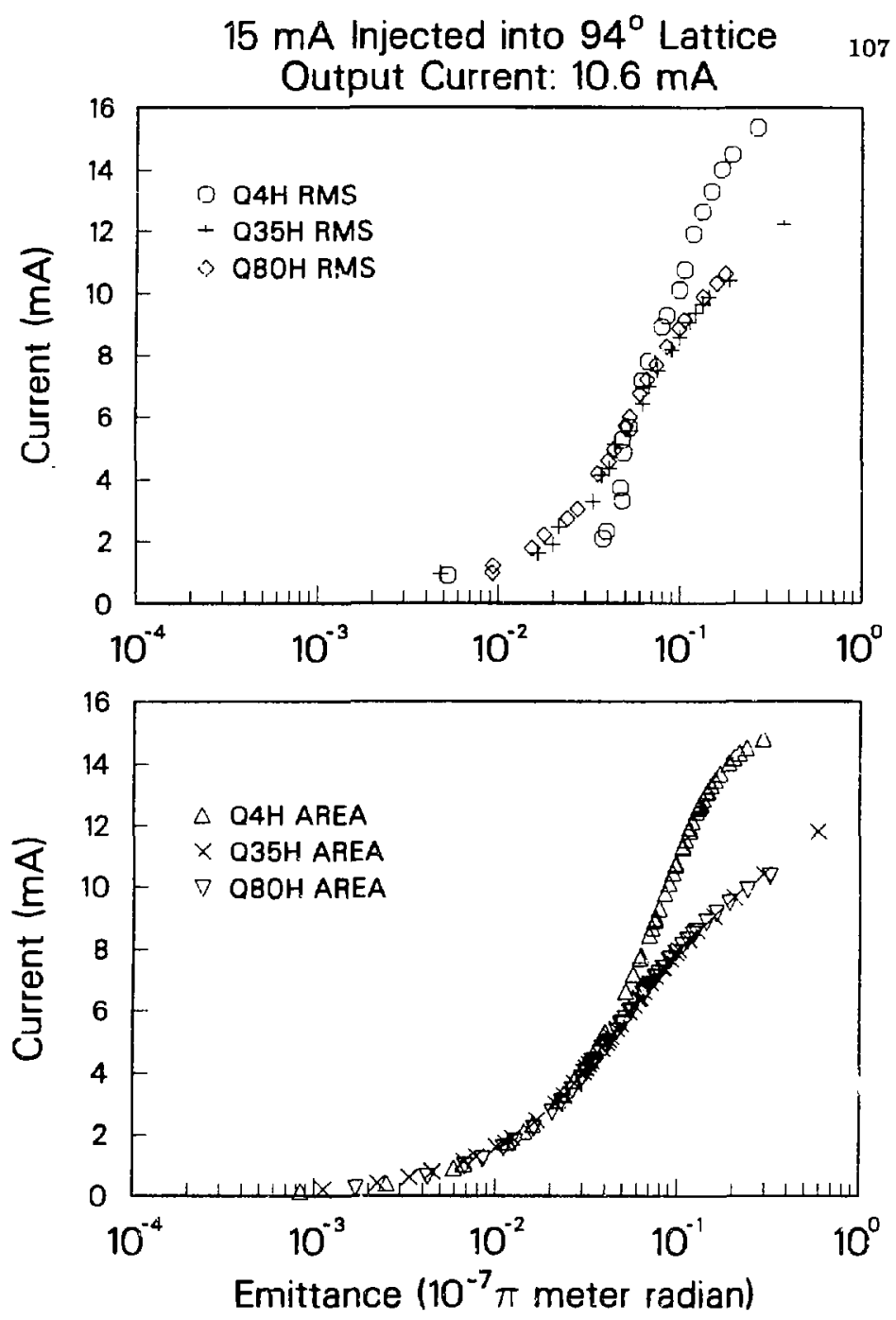

Figure 5.28: Current vs. emittance for $\sigma_{0}=94^{\circ}$, unstable beam. The core of the beam is little affected during the instability. Most of the change takes place in the outer phase space areas, with the change nearly complete by Q35. Downstream of Q35, the only change is an additional loss of about 2 $\mathrm{mA}$ of beam from the phase space tails. 

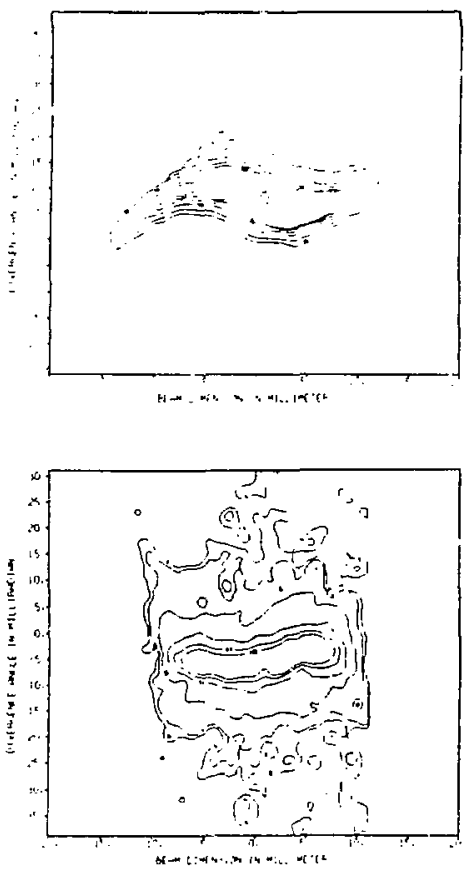

Q4

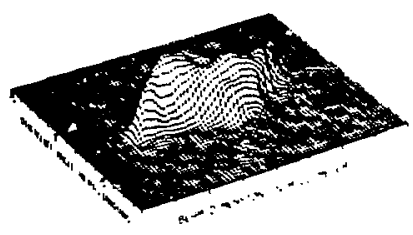

Q35
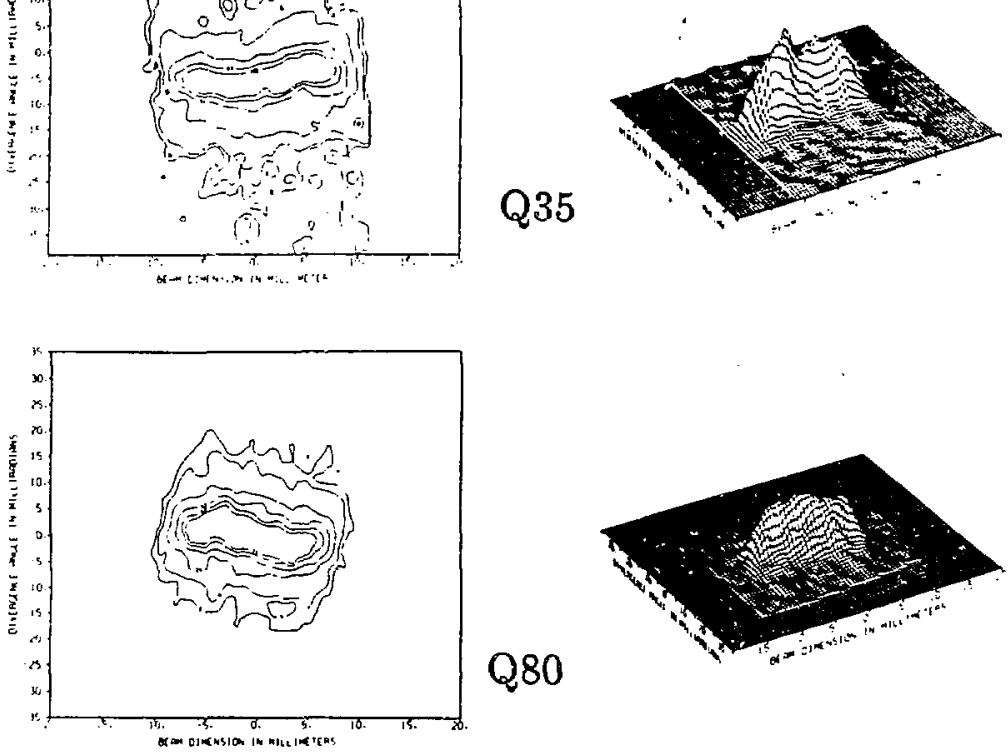

Q80

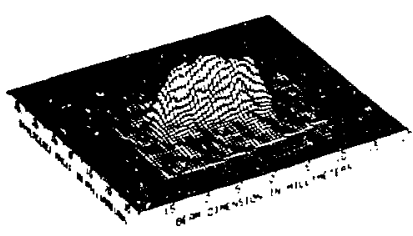

XBL $865-1831$

Figure 5.29: Instable beam behavior for $\sigma_{0}=94^{\circ}$. The beam has lost about $2.5 \mathrm{~mA}$ of the initial $15 \mathrm{~mA}$ by the time it reaches Q35. Extensive tails in phase space have developed, which are shed as the beam progresses on to Q30. 
rate of the instability near this value of $\sigma_{0}$ is only $4.3 \pm 1.9 \%$ per focusing period. This instability is in RMS radius, not in emittance, and the emittance should remain constant until the instability saturates through some nonlinear mechanism. Though the ends of the beam might be near the region of the envelope instability, the value of $\sigma$ for the bulk of the beam was a factor of at least three below the instability band for this particular mode, yet the beam emittance and current were rapidly degraded. Aiter the initial sross disturbance to the beam, which occurred during the first 15 lattice periods, subsequent measurements indicated th the beam almost exclusively lost particles in the fringes of the phase space distribution. The initial current was $15.2 \mathrm{~mA}$, and the final current was $10.6 \mathrm{~mA}$ at the Q82 Faraday cup.

\subsection{Discussion for $\sigma_{0}$ much greater than $90^{\circ}$}

For $\sigma_{0} \geq 118^{\circ}$, the beam stabilizes with an emittance significantly above that required to guarantee envelope stability, by a factor of 1.8 for $\sigma_{0}=134^{\circ}$. Recall that $\sigma$ increases monotonically with $\epsilon$. We found that when we injected too low an emittance, the resulting output emittance overshot the siability threshold, with loss of beam if the aperture clearance was insufficient. Upon raising the injection emittance, the output emittance decreased, with any beam loss diminishing at the same time. Increasing the injection emittance beyond the point at which the injection and output values met caused the output emittance to rise again, as expected.

For $\sigma_{0} \geq 134^{\circ}$, we were unable to transport even $3.5 \mathrm{~mA}$ through the entire lattice with no loss of beam. We were able to limit the beam loss to about $20 \%$ for $\sigma_{0}=134^{\circ}$ with a $3.4 \mathrm{~mA}$ beam, this loss occurring withir the first 15 periods of transport. The beam distribution was stable over the remaining 22 periods of transport for this case, with a measured output current of 2.7 $\mathrm{mA}$. For $\sigma_{0}=145^{\circ}$, the beam loss was slightly greater for the same injection 
conditions. The output current was $2.5 \mathrm{~mA}$, with a total emittance of about $2.8 \times 10^{-7} \pi$ meter radian and with an emittance for the central $95 \%$ of the beam of about $2.4 \times 10^{-7} \pi$ meter radian.

When plotted using the parameters $\sigma_{0}$ and $\sigma$, the empirical stability boundary is fit closely by a hyperbola of the form

$$
\sigma^{2}=\sigma_{0}^{2}-\text { const. }
$$

From smooth-focusing lattice solutions to matched beams, using the $\mathrm{K}-\mathrm{V}$ distribution, we find a similar relation between $\sigma$ and $\sigma_{0}$, derived from Eqn. 2.7

$$
\sigma^{2}=\sigma_{0}^{2}-\frac{1}{2}\left(\frac{180^{\circ}}{\pi} \frac{2 L}{v_{x}} \omega_{\mathrm{p}}\right)^{2}
$$

where

$$
\omega_{\mathrm{p}}^{2}=\frac{n q^{2}}{m \epsilon_{0}} .
$$

Here, $n$ the number density of the beam, $q$ the particle charge, and $m$ the particle mass. The constant value for $\omega_{p}$ which gives a good fit to the empirical stability boundary is given by

$$
\omega_{p} \simeq \frac{2 \pi}{3} \frac{v_{\pi}}{2 L}
$$

corresponding to one plasma wave oscillation while the beam transits three lens periods. Using this value in Eqn. 5.3, the $x$-intercept occurs for $\sigma_{0}=$ $120^{\circ} / \sqrt{2} \simeq 85^{\circ}$. Whether this correlation is indicative of a limiting beam "plasma frequency", we cannot definitively answer. However, we have estimated values for the beam density on the axis from our data by the following procedure.

In the low-emittance limit, there is little difference between the current profiles calculated from the $\mathrm{K}-\mathrm{V}$ (uniform beam) model and from solutions for 
a Maxwellian beam distribution (see Eqn. 5.1) for a smooth-focusing channel. For hot beams, however, the current profiles of a K-V beam and a Maxwellian beam are quite different. The Maxwellian has a local current density peaked at the beam axis, while the $\mathrm{K}-\mathrm{V}$ beam retains its uniform current density. The current density for a uniform density circular beam may be calculated from the projection onto one spatial dimension as

$$
J_{U}=\frac{I}{4 \pi \tilde{x}^{2}}
$$

For a Maxwellian beam in which the self-field is not dominant over the emittance term in the envelope equations, the distribution in real space takes the Gaussian form

$$
n(x) \propto \exp \left(\frac{-x^{2}}{2 \tilde{x}^{2}}\right)
$$

where $\tilde{x}$ is the RMS radius in the $x$ dimension. For this distribution, the peak current density (on-axis) is twice that for a uniform distribution with the same current and RMS beam radius

$$
J_{G}=\frac{I}{2 \pi \tilde{x}^{2}}
$$

We will take these two expressions, $J_{V}$ and $J_{G}$, as estimates for bounds on the peak cursent density of our beams for the $\sigma_{0}>100^{\circ}$ region with emittances high enough to bring the beam out of the space-charge dominated regime. The results are shown in Fig. 5.30. In the range $\sigma_{0}<88^{\circ}$, the beann emittance is very low and the current density in the beam should be very close to uniform. In this region, the current density will fall along the line calculated for ideal lattices and low transverse kinetic energy. However, for $\sigma_{0}>120^{\circ}$, for which the emittance term in the envelope equations is much higher relative to the current term than for low- $\sigma_{0}$, the beams may be expected to be 
nearly Gaussian in profile because of the dominant effect of the emittance in the envelope equations. In this approximation, the beam parameters are consistent with having a number density limited by a fixed value. There is, of course, a transition region between the two extremes. While this is by no means compelling evidence, it is consistent with the empirical relation between $\sigma$ and $\sigma_{0}$ found in our experiments.

\subsection{Summary of Results}

We have measured the emittance and current of a beam with intense space-charge fields, at various positions along a quadrupole channel consisting of 41 FODO periods, plus five matching lenses. and have characterized the empirical limits of stability, defined empirically as the conservation of the beam current and emittance along the channel. We have summarized the results graphically in Fig. 5.11 in terms of the quantities $\sigma_{0}$ and $\sigma$. We also present these same data in terms of $\sigma_{0}$ and the ratio $\sigma / \sigma_{0}$ in Fig. 5.31, compared with the early conjecture by Maschke and the later estimate by [HHLS $]$ of possible limiting values of the quantity $\sigma / \sigma_{0}$.

1. For low focusing strength $\left(\sigma_{0}<88^{\circ}\right)$ we have observed no collective limit to low-emittance beam transport in an A.G. lattice. Our source has an intrinsic minimum emittance, which places a lower bound on the value of $\sigma$ accessible at a given lattice strength. The only increase in emittance we have observed in the SBTE for $\sigma_{0}<88^{\circ}$ has been associated with aberrations in the phase space distribution of the beam, which were dependent on the matching section configuration. We believe that the matching section field is responsible for these aberrations, rather than any collective beam interaction with the lattice. (Interaction of the beam with the lattice through induced charge on nearby conductors provides measurable effects on the beam (see section 6.2), 


\section{Approximate current density in SBTE as a function of $\sigma_{0}$}

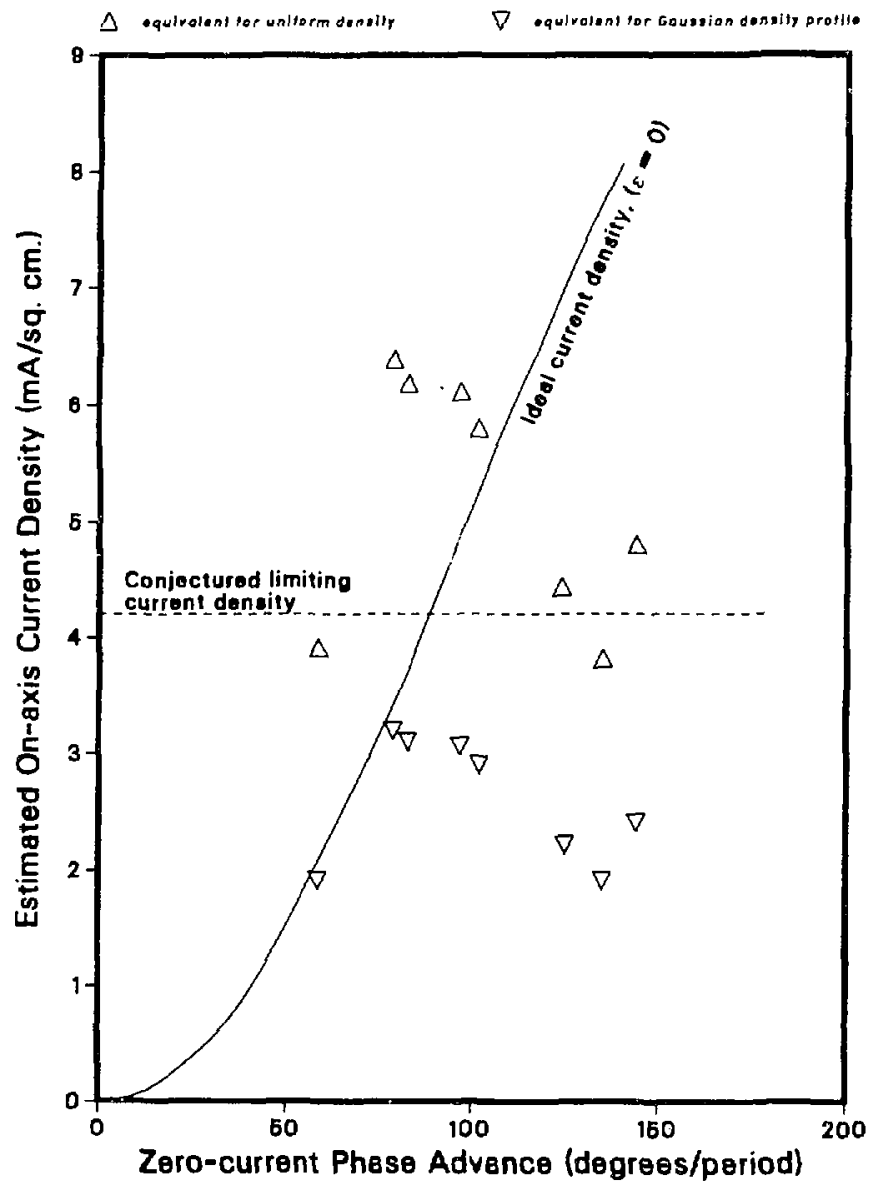

Figure 5.30: Bounds for peak current density of the beam near the stability boundary. For $\sigma_{0} \leq 88^{\circ}$, the beam is cold enough to treat in the uniform density limit, in which the current density is $I / 4 \pi \tilde{x}^{2}$. For $\sigma_{0} \geq 124^{\circ}$, however, the emittance dominates, and the beam is better approximated as Gaussian in current density. In this limit, the peak current density of the beam is $I / 2 \pi \tilde{z}^{2}$. We have plotted these two quantities for the beam parameters listed in Table 5.1. See text for discussion. 


\section{Stability limit summary plotted as $\sigma / \sigma_{0}$ vs. $\sigma_{0}$}

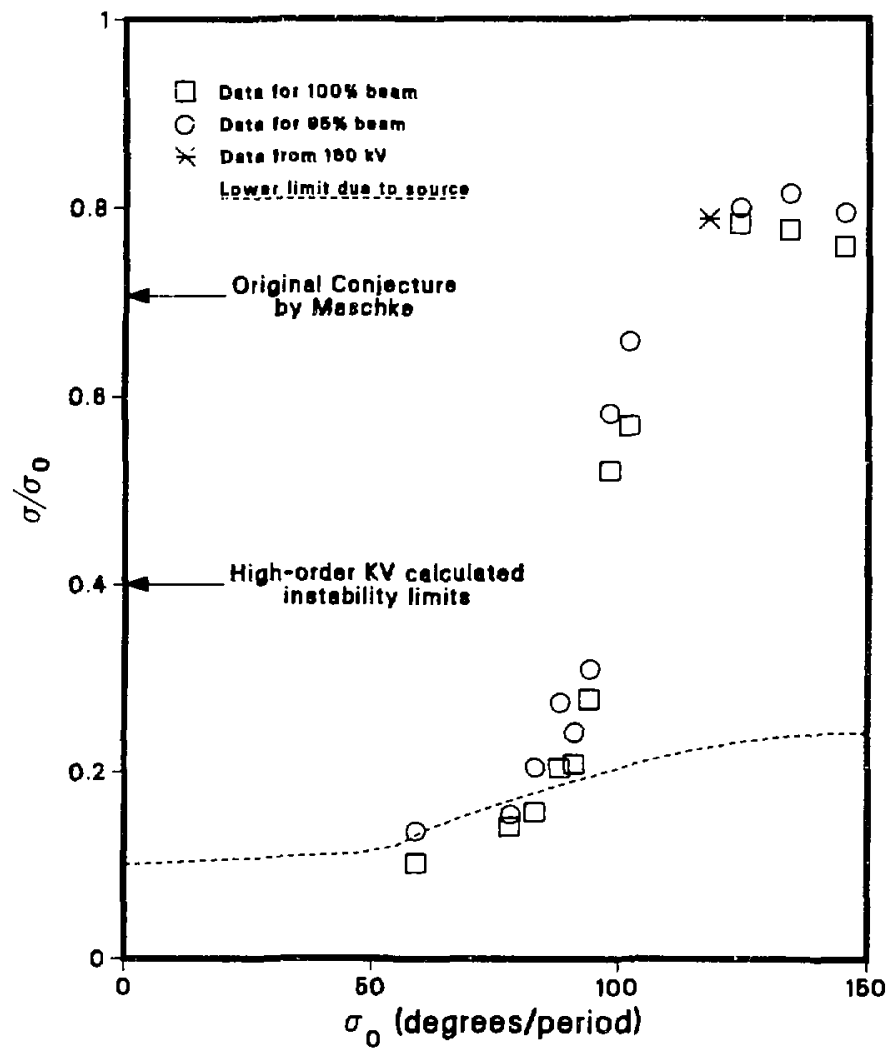

XBL $865-1838$

Figure 5.31: Results of the stability measurements from the SBTE using the parameters $\sigma / \sigma_{0}$ and $\sigma_{0}$. The early conjecture by Maschke $\left(\sigma / \sigma_{0} \geq 0.7\right)$ underestimates the beam intensity attainable in the SBTE. The later estimate $\left(\sigma / \sigma_{0} \geq 0.4\right)$, based on results for $\mathrm{K}-\mathrm{V}$ beams [20], is also somewhat conservative for $\sigma_{0}<88^{\circ}$. In this region, our lower limit on the attainable value of $\sigma / \sigma_{0}$ is given by the intrinsic emittance of the SBTE ion source, rather than by collective effects. See the text for more discussion. 
and this mechanism may provide the practical low- $\epsilon$ limit on spacecharge dominated beam transport.)

2. For $\sigma_{0}>8^{\circ}$, we observed intensity-dependent beam degradation. The mechanism is not certain, and we have seen definite structure in the phase space distribution of the beam to signal a particular mode of instability only for $\sigma_{0}=98^{\circ}$ and $\sigma_{0}=102^{\circ}$. In the unstable behavior in simulation work $[45,46]$, such structures are time-dependent, and we may have measured the beam distribution at an unfortunate choice of points along the channel. It is more probable, however, that we have not seen these mode structures because in the measurements we have made, we have generally attempted to inject the beam with parameters for which we expect only mild instability.

3. When we reach $\sigma_{0}=88^{\circ}$ without attenuating our beam, we find a small beam loss $(\sim 4 \%)$. Further increases in $\sigma_{0}$ result in a dramatic increase in the emittance of the beam and much greater loss of current. This threshold in $\sigma_{0}$ for growth of the beam emittance corresponds fairly closely to the threshold strength for envelope instability, but the detailed beam behavior is not consistent with this as the limiting phenomenon. The beam becomes unstable for parameters well outside the intensity band for the envelope instability and stabilizes while remaining on the high-intensity side of the unstable band. The growth rates calculated from perturbation of the envelope equations are inconsistent with the rapidity of the observed beam degradation in the neighborhood of $\sigma_{0} \sim 90^{\circ}$. For $\sigma_{0}>118^{\circ}$, the beam became stable only for an emittance much greater than that required to bring the beam to the low-intensity side of the envelope instability band, by a factor of about 2 for $\sigma_{0} \geq 134^{\circ}$. 
4. Although our experimental data are not well-correlated with the envelope stability curve, they are well represented by the smooth approximation relation

$$
\sigma^{2}=\sigma_{0}^{2}-\frac{1}{2}\left(\frac{180^{\circ}}{\pi} \frac{2 L}{v_{x}} \omega_{p}\right)^{2}
$$

where $2 L \omega_{\mathrm{p}} / v_{\mathrm{a}}$ is a constant approximately equal to $2 \pi / 3$. This is the hyperbola plotted along with the data. The $x$-intercept of this curve is at $120^{\circ} / \sqrt{2} \simeq 85^{\circ}$. For emittances corresponding to values of $\sigma$ and $\sigma_{0}$ below this curve, there is a violent instability in the phase space distribution of the beam. We present this as an observation on the beam parameters correlated with the cessation of this violent instability. We find no significant growth of emittance for values of $\sigma$ and $\sigma_{0}$ above this curve within the available transport channel length (82 quadrupoles plus the 5 matching lenses), with the exception of the region bounded by the instability curves for the envelope mode in Fig. 5.11. 


\section{Chapter 6: Other Experiments}

\subsection{Space-charge Coupling of Transverse Dimensions}

Liouville's theorem states that for a Hamiltonian system of $\mathrm{N}$ particles, the $6 \mathrm{~N}$-dimensional phase space density is a constant of the motion. For non-interacting particles, one may integrate over all but one of the particles and obtain a single-particle 6-dimensional distribution function, which is also a constant of the motion. For charged particles, inclusion of the self-fields complicates matters because of direct collisions between particles. In this experiment, discrete collisional effects are entirely negligible, and the distribution may be described in a 6-dimensional phase space.

To the degree that the spatial dimensions are decoupled, each may be described by averaging over the others and obtaining 2-dimensional distributions. This is standard practice in accelerator physics. The longitudinal dimension is usually decoupled from the transverse dimensions, and the two transverse dimensions are independent of each other tc the degree that the lenses are linear and orthogonally well aligned. The space-charge coupling of the beam radius in the two dimensions does not affect this condition in the ideal, linear-field case. However, non-linear space-charge fields can provide coupling in phase space. (Such coupling between dimensions is introduced externally in electron storage rings, through use of skew quadrupoles, to communicate the cooling effects of synchrotron radiation between the horizontal and vertical planes.) In the case of uncorrelated distributions in the $x$ and $y$ planes, the product of the emittances in the two planes should either be constant or grow somewhat.

That space-charge fields can provide this coupling is demonstrated by the following experiment. A single parallel-wire terminating grid had been placed at the ion gun output, with the wires oriented at $45^{\circ}$ from the quadrupole 
planes. The emittances in the vertical and horizontal planes were measured along the lattice and found to be equal at each lattice position and approximately constant in $z$. We then oriented the wires horizontally, and repeated the measurements. The field due to the image charges on the grounded grid wires was almost exclusively in the vertical plane, so that the emittance in the vertical plane received the entire grid perturbation, while the horizontal dimension retained the intrinsic source emittance. (A detailed discussion of grid effects on emittance is given in Appendix C.) The ratio of the emittances in the two planes as measured after $Q 4$, a short distance downstream from the source, was about $2: 1$, vertical:horizontal.

The results of the experiment are shown in Figs. 6.1 and 6.2. The emittances in the two planes are coupled, equilibrating with a characteristic distance of approximately 15 periods or less. The emittances as measured at $\mathrm{Q35}$, about 15 periods downstream from the $\mathrm{Q} 4$ measurement point, are nearly equal. The emittance in the vertical plane is still somewhat larger than that in the horizontal plane. But by the time the beam arrives at the end of the lattice, the emittances in the two planes are equal. In the absence of a strong $x-y$ asymmetric instability, then, we expect that $\epsilon_{x}$ and $\epsilon_{y}$ will be nearly equal for space-charge dominated beam transport. This may affect any HIF scenario involving beam merging.

If the interaction redistributes the beam transverse kinetic energy, without coupling in any of the electrostatic or longitudinal kinetic energy, then the sum of the squares of the RMS emittances will be constant. In this case, then the product of the two emittances will grow. However, because the initial ratio of the emittances in the two planes is only about 2 , we cannot distinguish between the conservation of the product, simple sum, or the sum of the squares of the emittances in the two planes. Conservation of these quantities, respectively, for an initial ratio between the $x$ and $y$ emittances 


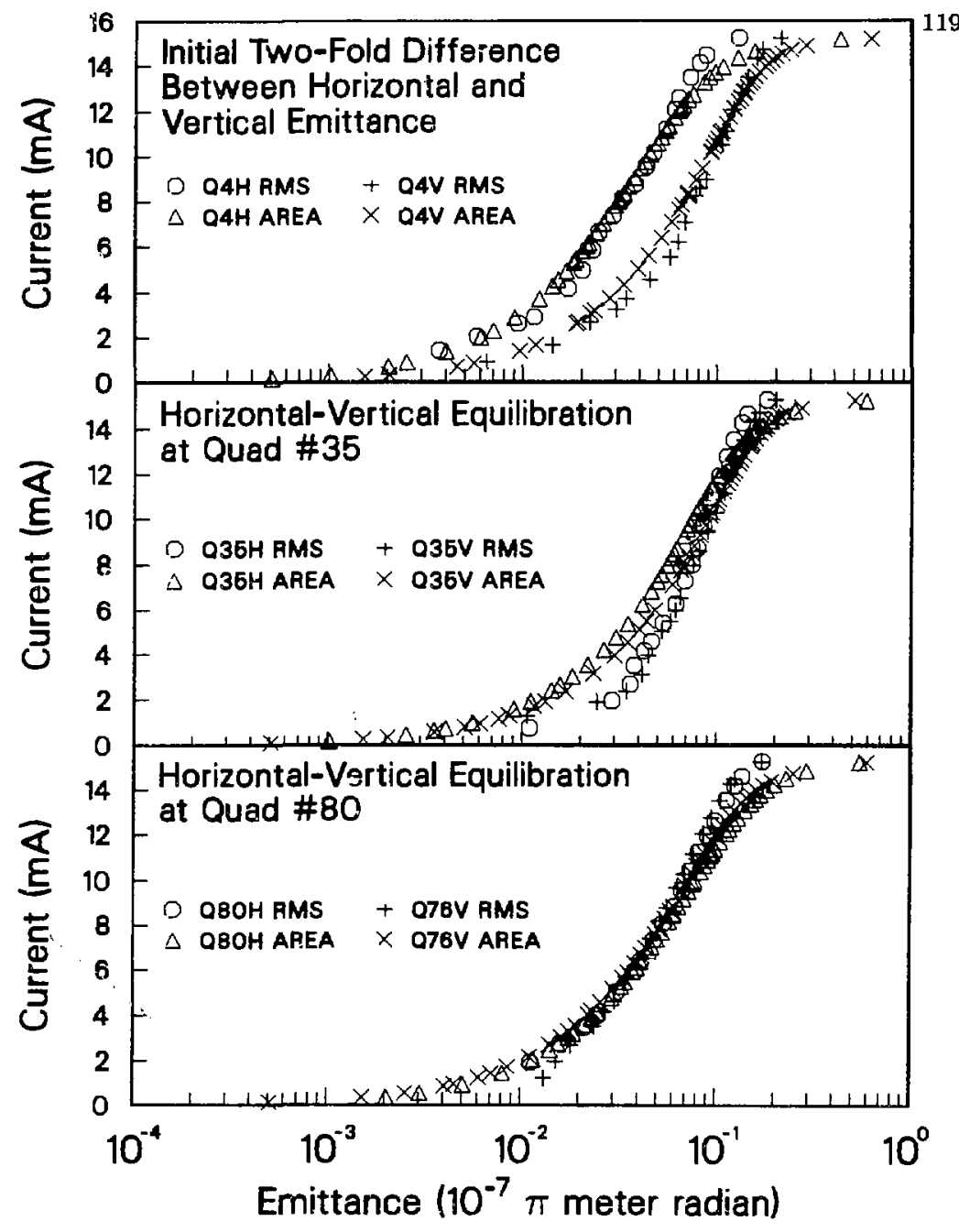

$\times C G 864-7160$

Figure 6.1: Horizontal-vertical emittance equilibration experimental results: (a) At Q4, the beam has almost a factor of 2 higher emittance in the vertical plane than in the horizontal plane. (b) By the time the beam has arrived at Q35, the transverse emittances are nearly equal. (c) The vertical emittance measured at Q76 and the horizontal emittance measured at Q80 are equal. 

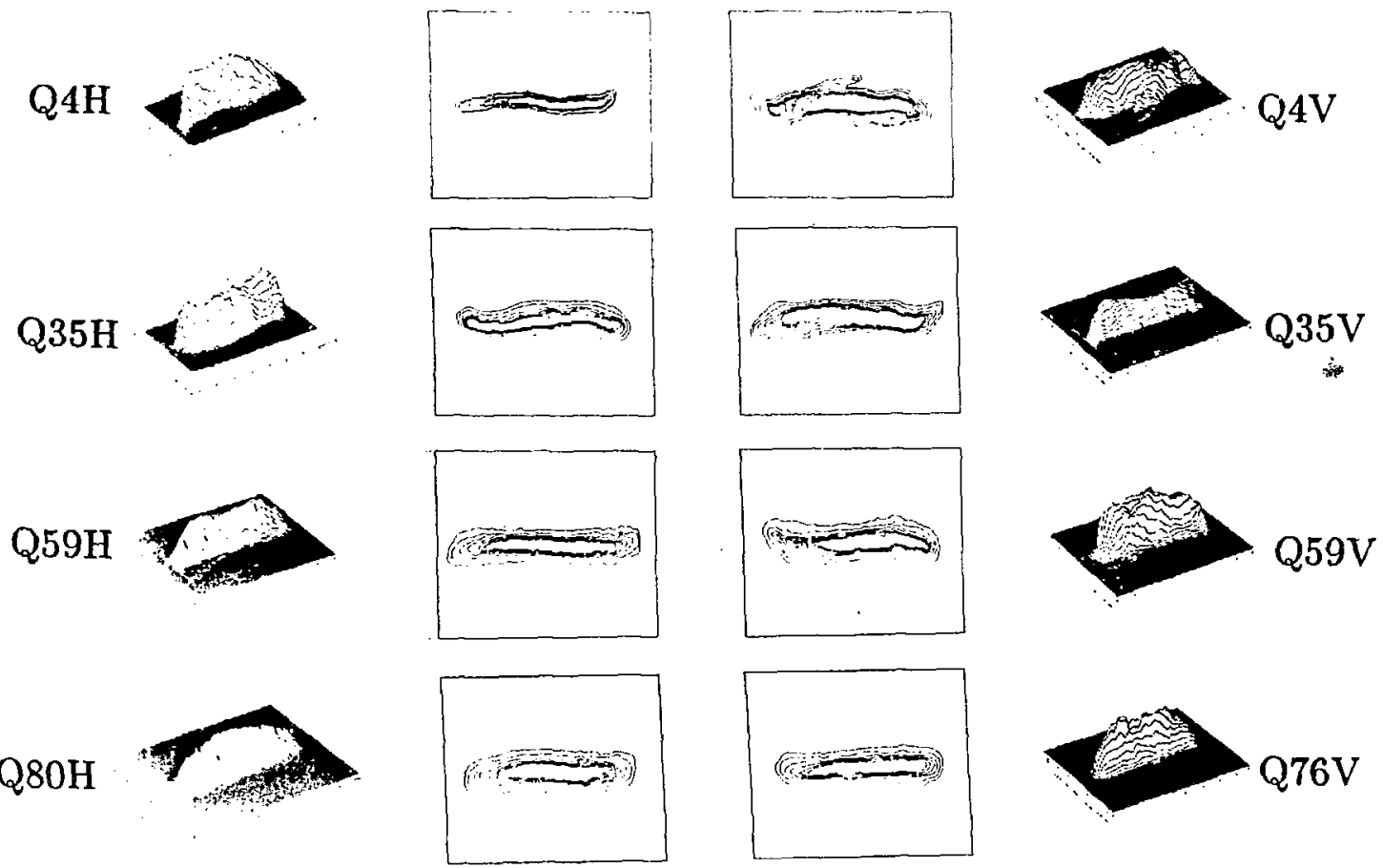

XBL $865-1749$

Figure 6.2: Phase space contours at various positions along the SBTE. The two left-most columns show the horizontal dimension results, while the two right-most columns show the vertical dimension data. The interchange of transverse energy is essentially completed upstream of Q35. 
of 2 gives a final emittance in each plane of about 1.4, 1.5, or 1.6 times the smaller initial value. The final emittances in the two planes for the case of the horizontal grid orientation were equal to the emittances measured with the grid oriented at $45^{\circ}$ to the horizontal.

\subsection{Image Charge Effects}

\subsubsection{Shift in the cohesent oscillation frequency}

Other than collective instability of the phase space aistribution of the beam, the emittance of a beam may be raised by the effect of induced charges on the quadrupole electrodes. Simulation results by Celata et al. [41] have indicated that induced charges on the quadrupole electrodes can raise the beam emittance greatly in the case of a misaligned beam, oscillating within the beamline, and Haber has shown that even for a well-aligned beam, the induced charges can limit the usable aperture [48]. At low values of the emittarice, there is very little random thermal energy in the beam. The overall focusing provided by the lattice is almost totally cancelled by the spaci-charge defocusing. There is no real shielding of the beam interior by plasma-like redistribution of the particles, except fur the average focusing over the lattice period. The nearly laminar beam flow can be vulnerable to the disturbing effects of relatively small driving terms, such as lens nonlinearities and image-charge fields.

To address this issue of image effects and make available a check on the accuracy of induced charge calculations in simulations, we have performed two experiments with the SBTE. The first effect we consider is the gross deflecting field experienced by the beam as it moves off-axis in the lattice. The induced field is very nonlinear, as may be seen from a first-order calculation using a cylindrical pipe for the aperture boundary. Treating the beam as a line charge with an offset $d$ from the axis of the channel, there is an image line 
charge as in Fig. 6.3. The image charge is of the same inagnitude as the real charge, but with opposite sign, and lies a distance $R=a^{2} / d$ from the axis of the pipe, where the radius of the pipe is a. (The real, extended beam gives rise to an extended image of varying tharge density.) The resulting field can be represented in first order as that due to a line image. The resulting field may be decomposed in the frame of the bcam, into a dipole field (deflecting the beam) and higher-order multipoles, the magnitucies depending upon the beam offset. The dipole field, in particular, is a deflecting field of magnitude proportional to $d$ in the small beam displacement limit, and causes a decrease of the coherent beam phase advance rate below the single particle rate. We denote this coherent phase advance rate by $\sigma_{C}$. Within the approximations above, treating the image of the beam as a line charge imaged by a cylindrical pipe surrounding the beam, in a constant-focusing channel, the image-charge shifted value for the coherent beam "tune" is given by

$$
\sigma_{C}^{2}=\sigma_{0}^{2}-\left(2 L \frac{280}{\pi}\right)^{2} \frac{Q}{2 a^{2}}
$$

where $2 L$ is the focusing period asd $Q$ is the generalized perveance. In the zero-current limit, $\sigma_{C}=\sigma_{0}$.

In the same way as we measured the single particle $\sigma_{0}$ (section 4.2), we measured the image-charge shifted phase advance rate $\sigma_{C}$ for a $10-\mathrm{mA}$ beam. We again set up the beam to have a zero-crossing after Q5, as we had for the low-current beam, and repeated the sequence of measurements. The results are shown in Fig. 6.4. For a quadrupole setting of 7275 volts $\left(\sigma_{C}=59.2^{\circ} \pm 0.6^{\circ}\right.$ for the low-current, $0.35-\mathrm{mA}$ beam) we measured $\sigma_{C}=55.2^{\circ} \pm 0.6^{\circ}$ for a 10 $\mathrm{mA}$ beam. The shift in $\sigma_{C}$ due to the induced charges is thus $4^{\circ} \pm 1^{\circ}$ for $\sigma_{0} \sim 60^{\circ}$. The equivalent pipe radius which gives this same shift in the smooth focusing limit is about $34 \mathrm{~mm}$, somewhat larger than the $25.4 \mathrm{~mm}$ 


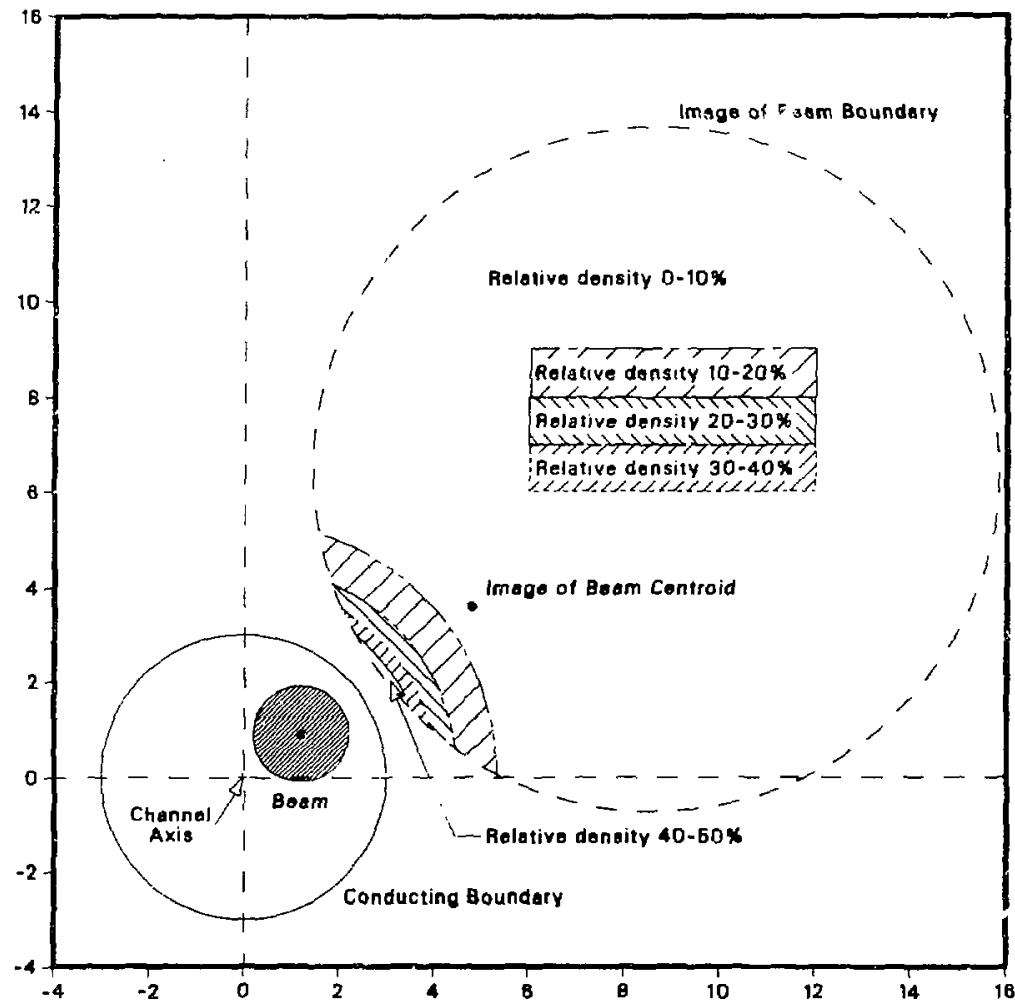

XBL $865-1842$

Figure 6.3: Model treatment of induced charge as due a line image. Image forces may be treated approximately by replacing the nearby conductors with a conducting cylindrical pipe of some effective radius, $a$, and approximating the beam as a line charge of $\lambda$ coulomb/meter. The image charge is then treated as an equivalent line charge, oi density $-\lambda$ coulombs/meter, a distance $a^{2} / d$ from the pipe axis, in the plane of the pipe wis and beam centroid. Here, $d$ is the beam offset from the pipe axis. The actual image of the beam is more complex, as shown in the figure; the charge density of the image beam varies widely over the area of the image. The dipole component of the image field in the frame of the beam centroid has the approximate magnitude $\left(\lambda / 2 \pi \epsilon_{0} a^{2}\right) d$, in the limit of $d$ much less than $a$. This provides a linear deflecting force, which reduces the overall restoring force of the channel. 


\section{Centroid vs. Potential for $\mathrm{Q79H}$ \\ $10 \mathrm{~mA}$ Beam}

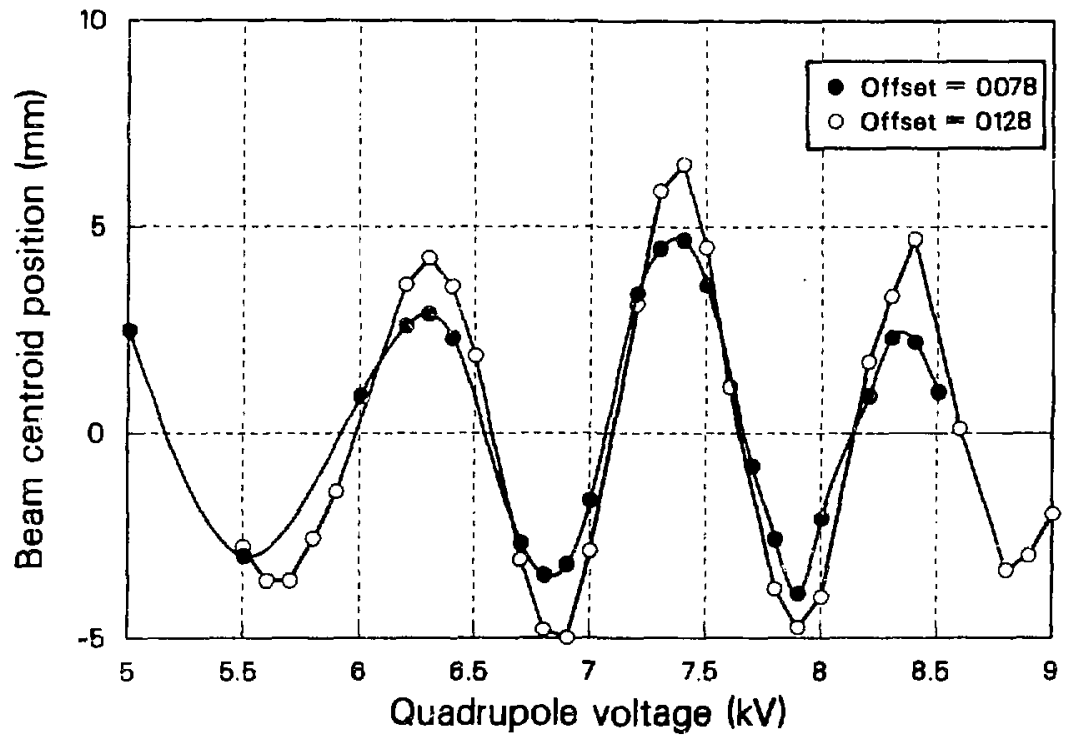

$X \cup 5864-7157$

Figure 6.4: Beam centroid vs. lattice strength for a high-current beam, for measurement of the induced-charge effect on the coherent beam oscillation. The overall phase of the coherent beam oscillation is retarded from that of the low current case shown in Fig. 4.2. The shift for $\sigma_{0}=59^{\circ}$ with respect to the low-current case in Fig. 4.2 is about $4^{\circ} \pm 1^{\circ}$, with a beam current of $10.3 \mathrm{~mA}$ and particle energy of $122.5 \mathrm{seV}$. 
inner radius of the SBTE bore.

\subsubsection{Emittance growth for off-axis beams}

The higher order image multipoles also perturb the beam. The quadrupole term in the beam frame augments the net focusing provided by the lattice, but this is of minor importance. In simulation work by Celata et al. with off-axis beams, an oscillation of the beam was seen to be driven by the sextupole image field [41]. They assume that the beam is offset from the axis of a perfectly aligned quadrupole transport system, with the boundary surfaces for the induced charges provided by continuous electrodes with the same longitudinal projection as the SBTE quadrupoles described in Appendix D. This sextupole field can drive a sextupole-symmetry space charge oscillation, the frequency of which in the low-emittance, low- $\sigma$ limit is degenerate with $\sigma_{0}[15]$. The resulting time-varying perturbation to the beam distribution causes the RMS emittance to oscillate and grow in the simulation, as seen in Fig. 6.5. The beating shown in the figure is due to the difference between $\sigma_{C}$ and the space-charge oscillation frequency. The simulations were run for $\sigma_{0}=60^{\circ}$, and the effect was sensitive to the value of $\sigma$, being very serious for $\sigma \leq 6^{\circ}$ and negligible for $\sigma \geq 12^{\circ}$.

Our experiment in the SBTE has found qualitative agreement with this emittance growth. The SBTE diagnostics are not closely spaced enough to observe an oscillation in phase space, if present. The best alignment we can provide for the beam results in approximately a $1.5 \mathrm{~mm}$ offset of the beam centroid in the channel. By moving the quadrupole doublet Q1-2 off the beamline axis by about 0.04 in (this provision is part of the mechanism allowing insertion of the M5 DFC), we displaced the SBTE beara enough to cause a few percent of beam loss. This beam displacement is estimated to be about $3 \mathrm{~mm}$. We compared the resulting $95 \%$ and $100 \%$ emittance 


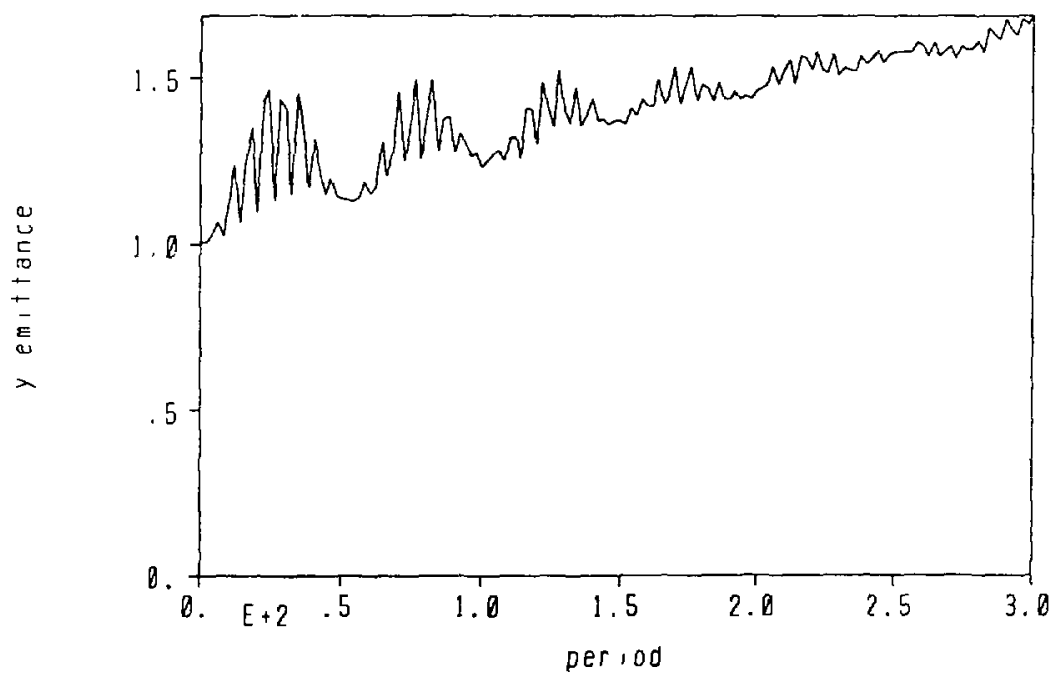

XBL $855-2333$

Figure 6.5: Simulation results for an offcenter high-current beam in a $\sigma_{0}=60^{\circ}$ lattice, after Celata et al. The oscillation in emittance is due to a sextupole-symmetry space-charge oscillation with a frequency in the low-emittance limit that is nearly degene rate with $\sigma_{0}$. The image field, oscillating at $\sigma_{C}$, drives the oscillation. The beating visible in the figure is a result of the difference between the space-charge oscillation frequency and $\sigma_{C}$. The oscillation and growth in emittance are not present in simulation of a well-aligned beam. 
values with those for the well-aligned beam, in both cases using the maximum current and minimum emittance attainable for us. The results are shown in Fig. 6.6. There is a small decrease in the apparent RMS emittance with $z$ for the better-aligned beam, as mentioned in section 5.1.4. But with the added offset, the emittance grows somewhat, ever while some beam is lost. The beam current was $15 \mathrm{~mA}$, with $2 \tilde{x}$ calculated to be about $19 \mathrm{~mm}$ at the center of a focusing lens, an estimated maximum beam offset of about $3 \mathrm{~mm}$ within a quadrupole, and a mismatch oscillation amplitude of about 1-2 mm. This essentially uses all of the $25.4 \mathrm{~mm}$ bore radius. The offset used for the experiment was the largest we could provide while avoiding beam loss greater than a few percent.

The resulting roughly $10 \%-20 \%$ growth in RMS emittance in the experiment is comparable to that in the simulation results. If one assumes that the boundary may be represented by a conducting pipe, then the perturbation of a beam in a smooth focusing channel by the sextupole driving field is dependent on the pipe radius $b$, the beam radius $a$, and the offset $h$ in the form $a^{3} h^{3} / b^{4}$, as shown in [41]. The expected perturbation in phase space, and consequently the emittance growth rate, depend strongly on the offset, which is not well-specified in the experiment. Because of the inevitable misalignments in the lenses, the phase and amplitude of the coherent beam oscillation will shift with respect to those for the perfect lattice alignment of the simulation. The relative phases of the space-charge oscillation and the coherent oscillation will therefore have a relative drift other than that which gives rise to the orderly beating of the simulation.

\subsection{Background Gas Stripping of $\mathrm{Cs}^{+}$}

Anoifur possible source of spurious beam loss and emittance growth in the SBTE is that the $\mathrm{Cs}^{+}$ions may change their charge state during collisions 


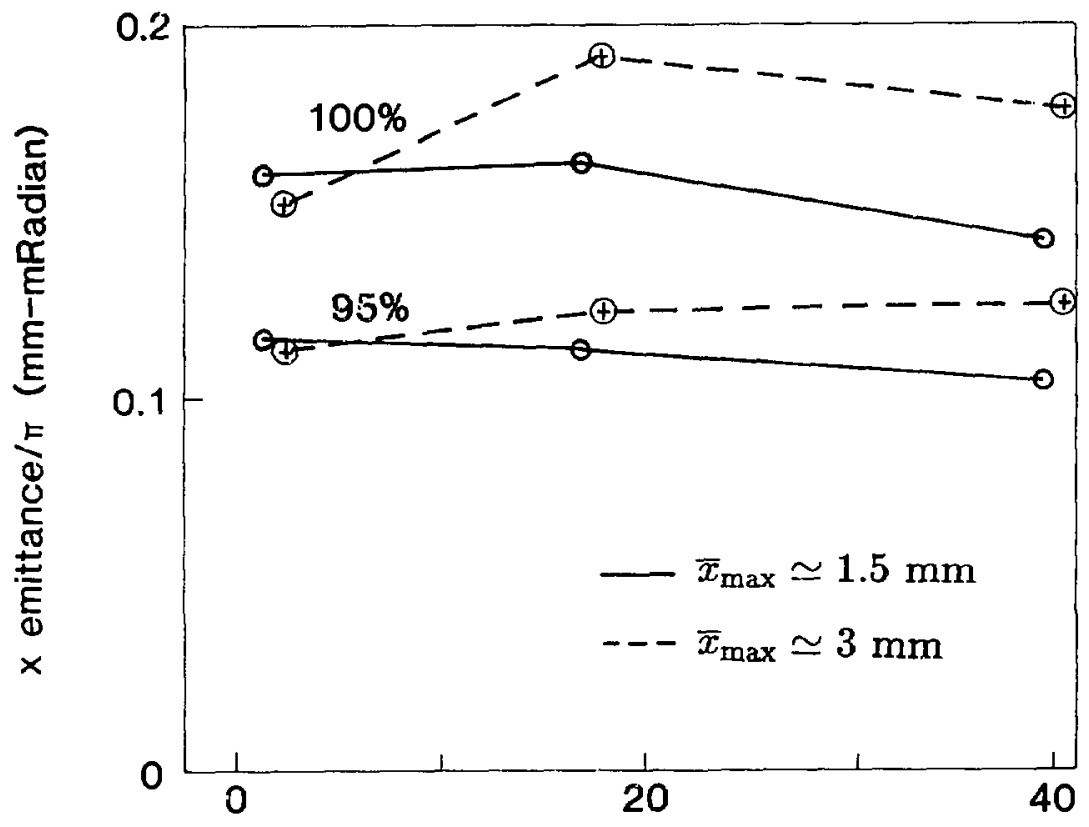

\section{Lattice Periods}

XBL $854-2195$

Figure 6.6: Experimental emittance growth for high-current, off-center beam. Sparseness of diagnostics would not permit detection of any oscillation in the emittance. The best alignment possible results in a beam offset within the bore of about $1.5 \mathrm{~mm}$, due to the lattice alignment errors. For the well-aligned beam, the measured emittance decreases slightly along the lattice. By shifting the Q1-2 doublet horizontally about $1 \mathrm{~mm}$ from its former position, we increase the offoet of the beam within the channel to about $3 \mathrm{~mm}$. We observe a few percent loss of current at the Q82 DFC, and a rise in the emittance of the remaining beam. The roughly $10-20 \%$ growth in einittance is consistent with the simulation result. 
with background gas molecules. Any neutrals will be lost to the beam, resulting in beam loss withou'; growth in the emittance. However, $\mathrm{Cs}^{+2}$ ions can be retained in the lattice under certain experimental conditions and be detected by the diagnostics. Because their orbits will be grossly different from those of the $\mathrm{Cs}^{+}$ions, retained $\mathrm{Cs}^{+2}$ ions will show up as a halo of particles in phase space. The result would be indistinguishable from a collective degradation of the $\mathrm{Cs}^{+}$beam emittanie, and we must determine the possible magnitude of this effect.

Typical stripping cross-sections are on the order of a few times $10^{-16} \mathrm{~cm}^{2}$ [49], and if we estimate the probability of stripping (or neutralization) over the length of SBTE $(l=13$ meter $)$ at a pressure of $5 \times 10^{-7}$ Torr, assuming that the cross-section is on the order of $\sigma \sim 10^{-16} \mathrm{~cm}^{2}$, we $\mathrm{t}$ btain $n \sigma l \sim .002$, where $n$ is the number density of the gas molecules and $l$ is the length of the channel. We may safely assume that the ions will undergo at most one charge-state changing collision, and we may use the transmitted beam current to determine the actual neutralization and stripping cross-sections (which we will denote, respectively, by $\sigma_{10}$ and $\sigma_{12}$ ).

For $\sigma_{0}$ somewhat greater than $60^{\circ}$ for a $\mathrm{Cs}^{+}$, the corresponding $\sigma_{0}$ for a $\mathrm{Cs}^{+2}$ is beyond the $180^{\circ}$ single-particle stability threshold, as may be seen from Fig. D.3 in Appendix D. The energy and transverse velocity of an ion change negligibly during stripping, though its charge doubles. This means that the $\mathrm{Cs}^{+2}$ suddenly experiences forces corresponding to a doubled lens strength, but with initial conditions appropriate for a $\mathrm{Cs}^{+}$. In the zerocurrent limit, these particles will be driven from the beam, and the current along the lattice will vary as

$$
I=I_{0} \exp \left[-\left(\sigma_{10}+\sigma_{12}\right) n z\right],
$$


where $\boldsymbol{z}$ is the distance along the channel. (However, in the presence of the signficant space-charge forces of an intense $\mathrm{Cs}^{+}$beam, many of the $\mathrm{Cs}^{+2}$ ions can be stable in the weakened net focusing field.) For much weaker focusing, with a beam clearance sufficient to accommodate the mismatched initial conditions for the $\mathrm{Cs}^{+2}$, these ions would be retained. The current in this regime would vary as

$$
I=I_{0} \exp \left[-\left(\sigma_{10}-\sigma_{12}\right) n z\right],
$$

because the $\sigma_{12}$ interactions increase the electrical current of the beam.

We measured transmitted current as a function of lattice voltage and gas pressure for low-current beams for both $160 \mathrm{keV}$ operation and $120 \mathrm{keV}$ operation. We varied the pressure by opening a leak valve connected to at hosphere. The measured cross sections thus are appropriate to approximately $80 \% \mathrm{~N}_{2}-20 \% \mathrm{O}_{2}$ gas. The results, in terms of fractional beam transmission for the other parameters, are shown in Figs. 6.7 and 6.8. From the slopes of the curves using a lattice strength above that for which the doubly-charged ions can remain in the lattice, we calculate the sum of the cross-sections $\sigma_{10}$ and $\sigma_{21}$. Note that in Fig. 6.7, the initial slopes of the very low quadrupole strength curves is positive, indicating that second ionization occurs more often than neutralization, even allowing for incomplete retention of the $\mathrm{Cs}^{+2}$ ions. The electrical current of the beam rises, even as the particle current drops.

For strengths far from that for which the beamlet was matched, this will be a lower current than measured for a matched beam. In Fig. 6.7 (120 $\mathrm{keV}$ ions), for a quadrupole voltage less than about $3 \mathrm{kV}$, only part of the beam is transported to the Q82 Faraday cup. If the $\mathrm{Cs}^{+}$ions are aperture limited, then so will the $\mathrm{Cs}^{+2}$ be, and the value we obtain for $\sigma_{10}+\sigma_{12}$ will be an underestimate. In this parameter range, the overlap of the $\mathrm{Cs}^{+}$and $\mathrm{Cs}^{+2}$ 


\section{Current Transmission Through SBTE as a Function of Pressure for Various Quadrupole Strengths}

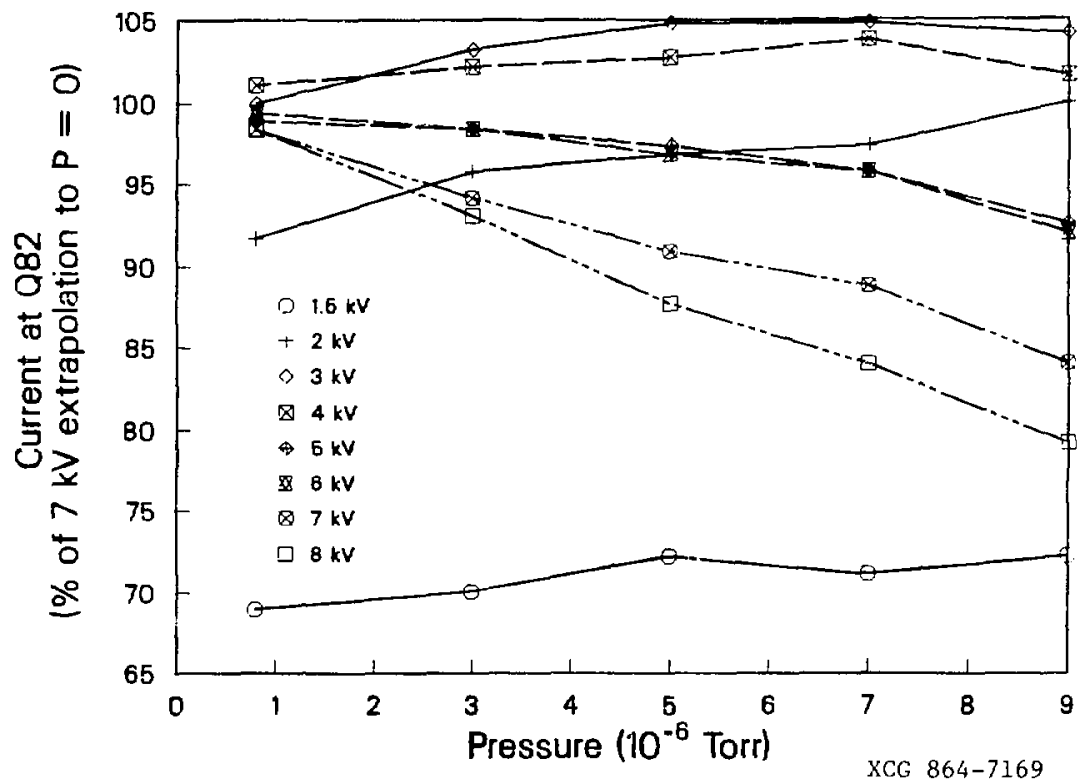

Figure 6.7: Current vs. pressure in SBTE lattice at $120 \mathrm{keV}$ particle energy. Second ionization of the $\mathrm{Cs}^{+}$ions causes loss of the ion for quadrupole voltages above about $7.5 \mathrm{kV}$, because $\sigma_{0}>180^{\circ}$ for the resulting $\mathrm{Cs}^{+2}$ ion. For lower quadrupole voltages, a $\mathrm{Cs}^{+2}$ ion may remain, if its transverse phase space coōrdinates fall within the acceptance curve for the new charge state. The exponential loss of ions from the beam from the combined processes of second ionization and neutralization may be calculated from the high voltage current vs. pressure curves. If the bore clearance is great, so that all the $\mathrm{Cs}^{+2}$ are retained, the low voltage curves give the exponential drop (or rise) in current as the electrical current is augmented by the $\mathrm{Cs}^{+3}$ ions. If the second ionization rate exceeds the neutralization rate, the current rises with pressure until the depopulation of $\mathrm{Cs}^{+2+}$ ions by other processes becomes important. The very low voltage curves for the $120 \mathrm{keV}$ ions show a distinct rise in current at the Q82 Faraday cup, as the pressure rises. This effect disappears for quadrupole voltages greater than about $4 \mathrm{kV}$. 


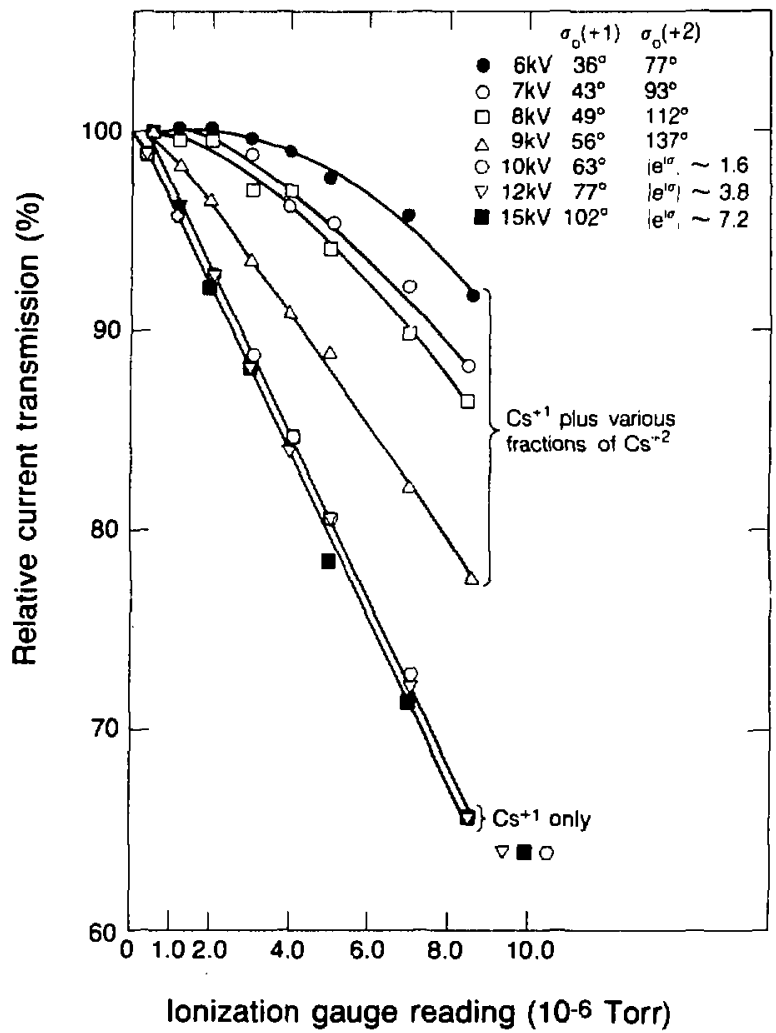

XBL. $844-10485$

Figure 6.8: Current vs. pressure in SBTE lattice at $160 \mathrm{keV}$ particle energy for various lattice strengths. The $6 \mathrm{kV}$ minimum quadrupole potential shown for $160 \mathrm{keV}$ ions corresponds to $4.5 \mathrm{kV}$ for $120 \mathrm{keV}$ ions. Based on the results for $120 \mathrm{keV}$ ions, our measurements for the $160 \mathrm{keV}$ case were not carried to quadrupole strengths low enough to measure the difference in the second ionization and neutralization cross-sections. We expect the difference in cross-sections to be comparable for $120 \mathrm{keV}$ and $160 \mathrm{keV}$ ions. 


Measured cross sections $\left(10^{-16} \mathrm{~cm}^{2}\right)$
\begin{tabular}{|c|c|c|}
\hline$\sigma_{12}+\sigma_{10}$ & $5.2 \pm 0.5$ & $9.2 \pm 0.7$ \\
\hline$\sigma_{12}-\sigma_{10}$ & $2.0 \pm 0.7$ & $2 \pm 2$ (est.) \\
\hline$\sigma_{12}$ & $3.6 \pm 0.6$ & $5.6 \pm 1.4$ \\
\hline$\sigma_{10}$ & $1.5 \pm 0.6$ & $3.6 \pm 1.4$ \\
\hline
\end{tabular}

Table 6.1: Measured cross-sections for charge-changing collisions for 120 and $160 \mathrm{keV} \mathrm{Cs}^{+}$ions against air.

acceptances of the lattice is good, so most of the stripped ions will be retained. For $V_{Q} \sim 4 \mathrm{kV}$, the current becomes flat with pressure, because the overlap in the lattice acceptance for the two species decreases. By extrapolating the curves to zero pressure, we determine how much current would have been transmitted by the lattice in the absence of charge-changing collisions. This is the base current that should be used to calculate the relative change in current with pressure for each particular lattice voltage. We have instead plotted the curves as the fraction of the maximum zero-pressure beam current (about $0.37 \mathrm{~mA}$ ) to show the incomplete transmission for low lattice strength which results from severe mismatch of the beam. For the $160 \mathrm{keV}$ beam in Fig. 6.8, we did not extend the measurements to a low enough lattice strength to get a good measurement of the difference of the cross-sections, although we can measure the sum of the cross-sections. We estimate from the rough equality of the slopes of the $6 \mathrm{kV}$ quadrupole voltage for $160 \mathrm{keV}$ ions and of the equivalent line interpolated for $4.5 \mathrm{kV}$ quadrupole voltage and $120 \mathrm{keV}$ ions, that the difference of the cross-sections for $160 \mathrm{keV}$ ions is about the same as for the $120 \mathrm{keV}$ ions. Our final results are given in Table 6.1.

Using $9 \times 10^{-16} \mathrm{~cm}^{2}$ for the sum of the cross sections, we see that for an indicated pressure of $5 \times 10^{-7}$ Torr, at most $2 \%$ of the beam particles 
undergo a charge-state changing collision. This is small enough to neglect in our emittance measurements, particularly since we quote emittance values for the most intense $95 \%$ of the beam. 


\section{Chapter 7: Error Analysis}

We now list the sources of error which could affect our measurements and the resulting error level of each. These include

1. uncertainty in the correct value of $\sigma_{0}$ for the beam, resulting botin from energy and quadrupole calibration errors and from the nonlinearity of the focusing field.

2. omission of part of the beam from measurement,

3. longitudinal space-charge effects near the ends of the beam,

4. non-zero slit-size corrections,

5. slit misalignments with the quadrupole symometry planes,

6. space-charge forces in the drift region between sli's,

7. space-charge perturbations due to the presence of the slits in the beam,

8. pulse-to-pulse variation of the beam,

9. background gas collisions with beam ions, and

10. secondary electron yield variations.

We will examine each of these possible sources of error, estimating bounds for each, and at the end of this chapter will summarize the results.

\subsection{Errors in determiniscion of $\sigma_{0}$}

We have determined the beam energy to be $122.5 \pm 1 \mathrm{keV}$, and wo know the quadrupole voltage to within about $\pm 0.4 \%$. The relative uncertainty of aboul $1 \%$ between the beam energy and quadrupole voltage results in an uncertainty in the value of $\sigma_{0}$ for paraxial particles of about $0.5 \%$ near $\sigma_{0}=60^{\circ}$, 
increasing to about $1.3 \%$ near $\sigma_{0}=140^{\circ}$. In addition to this error, there is an uncertainty in the appropriate value for $\sigma_{0}$ because of the lattice nonlinearity. In order to estimate this uncertainty, we used the envelope equations to calculate the matched beam size in the SBTE lattice using the measured values of quadrupole voltage, emittance, and current. The maximum beam radii were all at least $10 \mathrm{~mm}$ within the focusing lenses. We then calculated $\sigma_{0}$ for single particles attsining a $10 \mathrm{~mm}$ maximum displacement. The values of $\sigma_{0}$ for paraxial particles calculated for a $1 \%$ relative error between the particle energy and the quadrupole voltage, unaking the lattice weaker, and those calculated for the voltage error in the opposite direction for particles attaining a $10 \mathrm{~mm}$ maximum excursion have been taken as the limits of uncertainty in the $\sigma_{0}$ value characterizing the lattice. More information on the nonlinearity of the lattice is given in Appendix D.

\subsection{Incomplete scans of phase space}

We compared the total beam current calculated from the phase space measurements against the cuirent as measured by the various Faraday cups to ensure that we had sampled the entire beam. The sum of the phase space measurements should be proportional to the total beam current, as follows. Wi denote the upstream and downstream siit widths by $w_{1}$ and $w_{2}$, respectively, and the scan increments by $\Delta_{1}$ and $\Delta_{3}$. During the measurements, only $w_{1} / \Delta_{1}$ of the beam particles will pass the first slit, and only $w_{2} / \Delta_{2}$ of these will pass through the second slit into the detector. If $\xi$ is the overall gain of the detector and $R$ is the resistance used as the oscilloscope termination, then

$$
\sum_{i j} f\left(x_{i}, x_{j}^{\prime}\right)=\xi \frac{w_{1}}{\Delta_{1}} \frac{w_{2}}{\Delta_{2}} I R
$$


where the values $f\left(x, x^{\prime}\right)$ are the measured signals. The intercalibration of the proportionality constants was done with the constant-current, $\sigma_{0}=60^{\circ}$, beam conditions used for cup calibration.

We calculated the intercepted current for each set of beam parameters subsequently used, and compared the values obtained with Faraday cup current measurements made along the lattice. In some cases, we failed to make the mid-lattice cup measurements, and have only the value of the current calculated from the emittance measurements. Because the cup-measured current and the current calculated from the slit measurements differ by 10$20 \%$ for most of the $\sigma_{0}>118^{\circ}$ beam measurements, there is an additional uncertainty in the values of beam current along the lattice for beams undergoing current loss. (That this lower current was not due to failure to scan over some of the beam-occupied phase space was shown by subsequent scans made over a much wider phase space area. The subsequent scans showed the same intercepted beam current as the first scans.)

There are two possible causes for the discrepancy. The first is that the beam may have been surrounded by a diffuse cloud of ions, driven from the beam by either collective interactions or the emittance grids, and having too low a density to be picked up distinctly by our diagnostics. We do not believe this to be the case, because the missing current would give an observable signal if spread out over the entire lattice acceptance. For $\sigma_{0} \sim 130^{\circ}$, the zero-current acceptance of the lattice (allowing particles to take up the entire $25.4 \mathrm{~mm}$ bore radius) is about $1 \times 10^{-9} \pi$ meter radian, unnormalized. Each two-slit sample point with 0.01 -inch slits at a 6 -inch spacing covers about $1.4 \times 10^{-7} \pi$ meter radian, or about $1.4 \times 10^{-4}$ of the total acceptance. If a typical "missing" beam current of $1 \mathrm{~mA}$ were spread out equally over the total lattice acceptance, there would be a background signal of at least .8 
$\mathrm{mV}$, or $(1 \mathrm{~mA}) \times\left(1.4 \times 10^{-4}\right) \times(500 \Omega) \times 12$ (the secondary emission gain factor). Because misalignuents restrict the available bore by several $\mathrm{mm}$ at this lattice strength, and because the missing current would have to be in the phase space not sampled in the measurements, the background level required to account for the $1 \mathrm{~mA}$ is really about $2 \mathrm{mV}$, well above the observed noise level of about $\pm 0.4 \mathrm{mV}$.

We thus conclude that thw most likely cause is the high divergence of the beam at high $\sigma_{0}$. Most of the scans for $\sigma_{0}>118^{\circ}$ were made in the convergent beam dimension. The source of the discrepancy with cup current measurements lies ir: the high divergence of the beam in the other plane. With the quadrupole between the first and second measurement slits grounded, some of the particles can lie outside of the acceptance of the slits, not in the plane of the measurement (say, the $x$ plane), but in the plane of the slits. See Fig. 7.1 for a graphic illustration of this effect. The quadrupole between the two slits must be grounded to make the measurement of $x^{\prime}$. If the beam divergence is marginally too large to allow the beamlet to fall entirely within the limits of the slit, then any misalignment or mismatch of the beam can cause part of the beam to miss the slit. Because of the linear optics and generally elliptical cross-section of the beam, particles with very large values of $y^{\prime}$ have associated large displacements in $y$, and they tend to have small offsets in $x$. If some of these particles were lost while measuring the phase space distribution in the $x$ dimension, the $f\left(x, x^{\prime}\right)$ signals would have been clipped near small $x$, where they are at their maximum. One can recover the correct measurement of phase area, but not the emittance ellipse shape and orientation, by turning the strength of the quadrupole just upst:eam of the primary slits to about one-half of its nominal value. In effect, this converts a highly divergent beam into a roughly parallel beam and allows the current to be directed into the area covered by the slit cups. We chose to leave the 
(A)

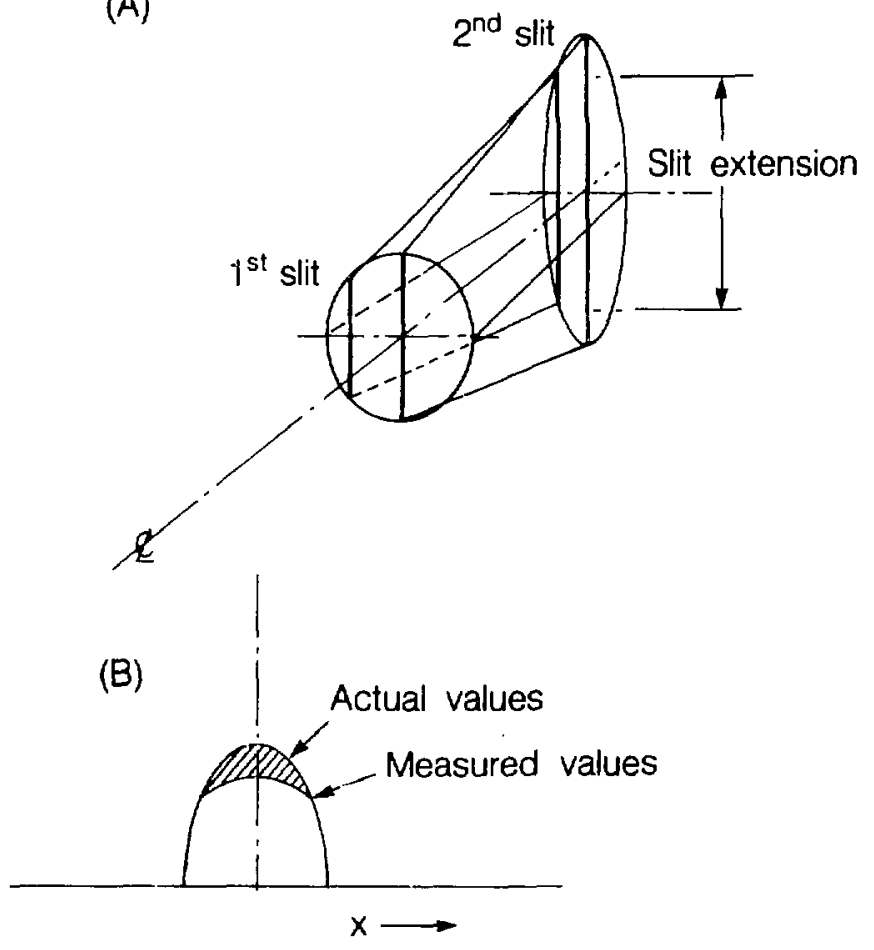

XBL B63-10351

Figure 7.1: When the beam is convergent in the plane perpendicular to the slits, the effect of the non-zero width of the first slit is minimized, as shown in section 7.4. The beam will not fall outside the measurement acceptance in the plane of the measurement. However, if the beamlet overfills the downstream slit in the divergent dimension, part of the current will be lost, causing too low a value for the phase space density at that $\left(x, x^{\prime}\right)$ point. The affected data tend to be near the center of the beam in the $x$ dimension, where the density is greatest. The RMS emittance calculated from the measured distribution will be too large, as a result. For our measurements, this error is estimated to be in the range $0-10 \%$. 
upstream quadrupole at the periodic lattice strength, to measure the irain radius at the same time as we measured the emittance.

This error lowers the measured phase space area and the phase space density near the beam core, but not at the beam edges. The effect depends on the phase and amplitude of the beam mismatch and misalignment oscillations, with no predictable severity at any location. We have estimated the effect of this error, using a correction factor for a beam of uniform density beam and elliptical cross-section, as in Fig. 7.1, of the form

$$
\max \left[1, \alpha \sqrt{\max \left[1-(x / 2 \tilde{x})^{2}, 0\right]}\right],
$$

where $\alpha$ is described below and $1 \tilde{x}$ is the RMS width of the distribution in the $x$ dimension. If an elliptical charge distribution of semi-axis $\alpha$ in $y$ is truncated by detection slits of unit half-length, the actual values for the distribution may be approximated as follows. We app:oximate the semi-axis in $x$ of the original distribution by $2 \tilde{x}$ from the measured distribution, and calculate the local half-height of the ellipse at a distance $x$ away from the $x$-centroid of the ellipse. We then multiply the measured phase space density values at that point in $x$ by the local height of the ellipse if it is greater than 1. For $\alpha \leq 1$, the dist-ibution is unaltered. As the value of $\alpha$ is raised, the total current in the corrected distribution rises, with values of the phase space density near the beam center raised preferentially. If the measured width of the distribution is close to the true width and the data are of the form assumed in Fig. 7.1, then the actual distribution in phass space is recovered for $\alpha$ chosen so that the corrected total current agrees with the Faraday cup measurements. Analysis of the corrected data yields marginally smaller emittance values than for the raw data, but the difference is no more than about $7 \%$ for $\epsilon_{95}$ and $\epsilon_{100}$. Thus the data are reliable, and we are able to place narrow bounds on the error from this mechanism in calculating the emittance of the beam. Although the 
beam sizes and divergences calculated from the envelope equations do not indicate that the beam should overfill the emittance scan slits as suggested above, beam misalignment could have allowed the beamlets to spill over one end of the downstream slit.

\subsection{Beam end-effects}

Our measurements were made near the center of a $10 \mu \mathrm{sec}$-long beam pulse (physical length about 4 meters). The beam rise and fall times at the ends are a fraction of a microsecond at the be ginning of the transport system and degrade (because of longitudinal space-charge effects) to about 2-3 $\mu \mathrm{sec}$ at the end of the channel. Hence the measurements we report are representative of an infinitely long beam. The development of local debunching at the beam ends along the lattice is apparent in Fig. 5.3. Control of the bunch ends is a separate research effort beyond the scope of this thesis.

\subsection{Effect of non-zero slit size}

We have calculated the effect on the emittance measurement due to the use of slits of non-zero width. The result (for a linearly focused beam) for the mean square width of a beamlet passing through a primary slit at $z=0$ and measured a distance $L$ downstream, is

$$
\left\langle x^{2}\right\rangle_{x=L}=L^{2} \tilde{\Theta}^{2}+m^{2} \frac{a^{2}}{12}+\frac{b^{2}}{12}
$$

where $\tilde{\Theta}$ is the local RMS angular width of the beam distribution at the first slit, $m$ is a magnification factor to be defined shortly, $a$ is the full width of the first slit, and $b$ is the full width of the second slit. To see this, we write the position and angular dependence of the beam distribution at the upstream slit in the form $f(x, p)$, where $p=x^{\prime}-s x$ and $s=-1 / f$. The parameter $s$ describes the focusing of the beam in terms of the distance $f$ to the geometric 
focus of the beam. The distance $f$ is positive for a convergent beam and negative for a divergent beam. This parameterization is used to separate the convergence of the beam from any other spatial or angular dependence. We will consider in detail only the effects of the non-zero size of the first slit. The second slit may be accounted for in a similar fashion.

A particle with coördinates $\left(x, x^{\prime}\right)$ at $z=0$ will have coördinates $(x+$ $\left.L x^{\prime}, x^{\prime}\right)$ at $z=L$, so

$$
\left\langle x^{2}\right\rangle_{2=L}=\left\langle\left(x+L x^{\prime}\right)^{2}\right\rangle_{x=0}=\left\langle(m x+L p)^{2}\right\rangle_{x=0},
$$

where $m=1+s L$ is the geometric size of the primary slit projected onto $z=L$ by the beam. For a beam distribution even in $p$, the cross term $x p$ averages to 0 , and the mean square beamlet size at $z=L$ reduces to averages of $x^{2}$ and $p^{2}$ over the collimated beati distribution at the first slit. We will irrite the slit distribution as $g(x)$, so that the collimated distribution of the beamlet at $z=0$ is $f(x, p) g(x)$. If we assume that the first slit is very narrow, then the collimated distribution may be written as the product of a purely angular dependence from the beam (apart from the focusing) and a spatial dependence from the slit, as

$$
f(x, p) g(x) \sim F(p) g(x)
$$

where $\mathrm{F}$ describes the angular distribution of the beam at the center of the slit. The averages then reduce to the form quoted in Eqn. 7.3. The higherorder corrections due to the variation of $f$ with position within the slit (apart from convergence, which we have handled properly) are of the order of the square of the ratio of the slit size to the beam diameter $\left(\sim 10^{-1}\right.$ in our case, cunsidered negligible). In the instance of a convergent beam, the effect of the nonzero size of the first slit disappears for $m=0$. This error contributes to an 
overestimate of the emittance and was checked by comparing measurements for the convergent and divergent planes for many of the beam configurations for $\sigma_{0}<100^{\circ}$. We saw no difference in emittance between the two planes of more than a few percent, except for the intentional asymmetry in emittance introduced for the measurements of section 6.1.

\subsection{Slit misalignment}

Slit misalignments with the each other and with the quadrupole symmetry planes increase the effective size of the slits. The alignment procedure used on SBTE relied on optical alignment of the slits with the same cross-hairs in a transit telescope that were used to set up the quadrupoles themselves. Slit parallelism was further checked by shining a light through both slits and checking the uniformity of illumination through the pair of slits with the telescope. The estimated misalignment between the ends of the 2 in-long slits was less than 0.005 in, making the effective width of the bearn-occupied portion of the slit at most 0.013 in. We included the effects of misalignment by using this allowance for the size of the slits.

\subsection{Space charge effects}

We have estimated the space-charge effects by integration of the envelope equations for the beamlet passed by the upstream slit. We included the overall convergence and divergence of the beam, using twice the RMS radius of the slit as the initial radius in one dimension, and twice the RMS radius of the beam as the beamlet radius in the other dimension. The current transmitted through a slit at the beam centroid was estimated from the phase space measurements, and the level of the error introduced by the space-charge was taken to be bounded by the ratio of the beamlet radius calculated in the presence of space-charge to the radius calculated for a zero-current beamlet. The correction varies from $1 \%$ to $4 \%$, with the actual beam emittance smaller 
than that calculated directly from the data.

An estimate for the correction may also be obtained by taking a zeroemittance model beam, and calculating the transverse field due to the spacecharge of the slab beamlet transmitted through the first slit. For particles at the beamlet edge, the electric feld may be taken as constant, apart from the convergence or divergence of the beam in the direction parallel to the slit, as

$$
\mathrm{E}_{\perp}=\frac{\rho d}{2 \epsilon_{0}}
$$

The space-charge deflection of the particles during the 6 in drift between slits may be easily calculated, and gives a bound on the error of a few percent in the beamlet size at the second slit, in agreement with the envelope equation calculations.

\subsection{Slit-beam perturbation}

Another effect on the beam related to space-charge forces alters the focusing properties of the beam near the first slit. The conducting slit shorts out the transverse self-field of the beam for a distance of about one beam radius from the slit, causing the beam to be less divergent than it would have been in the absence of the slit. The result is less than a $0.1 \mathrm{~mm}$ shift in beam size and a few milliradian shift in convergence angle. For example, for $I=15.2$ milliampere, $\sigma_{0}=83^{\circ}$, and $R_{\text {Beam }}=0.012$ meter, we have a local transverse self-field of $50 \mathrm{kV} /$ meter. This results in a change in beam radius on the order of $15 \mu \mathrm{m}$, or about $0.001 \mathrm{in}$. The change in divergence is more noticeable, giving $\Delta \theta \simeq 2.5$ milliradian at the beam edge, compared to a typical divergence angle at the beam edge of about 70 milliradian. This affects the emittance measurement only through the higher order effect of aberrations in the beam focusing, which we have considered to be negligible. 


\subsection{Pulse-to-pulse variation of the beam}

Overall reproducibility was good on a long-term basis, with particular reference conditions at $\sigma_{0}=60^{\circ}$ yielding reproducibility in total current intercepted in the two-slit scans and in the calculated RMS emittance at the $1 \%$ level over a period of months. However, for some of the $\sigma_{0}>100^{\circ}$ measurements, the variability was greater, resulting in values for the beam emittance estimated to be too large by as much as 6-8\%. This estimate was made by interpolating and smoothing the most seriously affected data by hand and comparing the total emittances before and after the smoothing.

\subsection{Background gas effects}

Because the electrostatic quadrupoles sweep free static charges from the bore, space-charge neutralization is unimportant for SBTE. Beam-gas nuclear collisions are negligible, and electronic interactions cannot supply enough momentum exchange to deflect the $\mathrm{Cs}^{+}$ions by significant angles. The chargestate changing interactions of electron capture and second ionization are the only effects of the background gas on the beam. Neutralized cesium ions are lost to the beam, but under certain circumstances $\mathrm{Cs}^{+2}$ ions may be retained and be counted twice in the current monitors.

The measured cross-sections for these interactions in the SBTE imply that at normal operating pressures in the neighborhood of $5 \times 10^{-7} \mathrm{Torr}$, only about $2 \%$ of the beam particles will undergo a charge-state changing interaction. See section 6.3 for the detailed measurements made with the SBTE. We found no effect on the beam emittance within the usual scatter of a few percent when we measured the emittance of the full-current, $\sigma_{0}=60^{\circ}$, beam over the range of pressure $1 \times 10^{-7}-2 \times 10^{-6}$ Torr, although the beam current had been attenuated by charge-exchange interactions at the higher pressure by about $7 \%$. 


\subsection{Secondary electron yield variations}

Secondary electron yields may vary somewhat over a surface, depending, for instance, upon variations in adsorbed gas coverage. The slit cup collectors are very limited in actual area used, the maximum area being about 2 in by 0.08 in. The slit cups are probably immune to secondary yield variations other than a uniform variation over the surface as a function of time, which we believe to be small. The materials used are the same as for the SFC's, which showed no perceptible variation in response over the duration of the measurements, and we saw good agreement of the integrated slit cup current with the Faraday cup current measurements for $\sigma_{0}<100^{\circ}$ throughout the experimental period.

\subsection{Error summary}

We have identified several (primarily systematic) sources of error. The amount of the error from each mecilanism varies with the dominance of the space-charge in the beam. In plotting the $i$ vs. $\epsilon(i)$ curves, as well as in the phase space contour curves, these errors have been ignored. However, in the quoted values of the emittances $\epsilon_{95}$ and $\epsilon_{100}$, the systematic errors have been corrected. In Table 7.1 we have summarized the effect of these errors, and indicated the typical correction resulting from them. The effect of these emittance corrections is small in the derived $\sigma_{100}$ and $\sigma_{95}$ depressed phase advance values. The error in these values is due primarily to the noise in the tails of the phase space distribution data, and we believe that the value of $\sigma$ representing the actual beam parameters lies within the bounds given by $\sigma_{100}$ and $\sigma_{95}$ in Fig. 5.11 and Table 5.1. 


\begin{tabular}{|c|c|c|}
\hline Mechanism & $\begin{array}{c}\text { Error } \\
\text { Bounds }\end{array}$ & Comments \\
\hline $\begin{array}{l}\text { Errors in lens voltage } \\
\text { Intrinsic lens nonlinearity }\end{array}$ & $\pm 1^{\circ}$ to $\pm 3^{\circ}$ & $\begin{array}{l}\text { Calculated for paraxial } \\
\text { partricles with } 1 \% \text { low lens } \\
\text { strength and for } 10 \mathrm{~mm} \\
\text { maximum orbit amplitude } \\
\text { for } 1 \% \text { high lens strength }\end{array}$ \\
\hline Collimating slits too short & $0-7 \%$ & $\begin{array}{l}\text { Makes measured values for } \\
\epsilon \text { too large, primarily for } \\
\sigma_{0}>100^{\circ}\end{array}$ \\
\hline $\begin{array}{l}\text { Nonzero slit size or } \\
\text { misaligned slits }\end{array}$ & $0.5-2 \%$ & $\begin{array}{l}\text { Measured values for } \epsilon \text { too } \\
\text { large }\end{array}$ \\
\hline $\begin{array}{l}\text { Nonzero space-charge } \\
\text { during drift between slits }\end{array}$ & $0.7-4 \%$ & More severe for $\sigma_{0}<100^{\circ}$ \\
\hline $\begin{array}{l}\text { Variation between pulses } \\
\text { during beam measurements }\end{array}$ & $0-6 \%$ & $\begin{array}{l}\text { Measured values for } \epsilon \text { too } \\
\text { large }\end{array}$ \\
\hline $\begin{array}{l}\text { Collisions of beam particles } \\
\text { with background gas } \\
\text { particles }\end{array}$ & $<3 \%$ & $\begin{array}{l}\text { Causes beam loss, with } \\
\text { effect on } \epsilon \text { dependent on } \\
\text { retention of second-ionized } \\
\text { particles. Major effect is } \\
1-2 \% \text { beam loss }\end{array}$ \\
\hline
\end{tabular}

Table 7.1: Summary of error bounds 


\section{Appendix A: Beam Dynamics}

In Chapter 2 we outlined a development of the dynamics of a particle beam from the single-particle trajectory equation without space charge forces, progressing through the envelope equations and the Courant-Snyder [26] invariant without space-charge forces. Space-charge was included first in a linear field model using the distribution of Kapchinskij and Vladimirskij [30], or the "K-V" distribution. In essence, the $\mathrm{K}-\mathrm{V}$ distribution allows the same handling of particle trajectories as the zero-current case because the spacecharge field behaves as a distributed linear lens. The particle beam envelope is then calculated self-consistently with its own space-charge field. This is a numerical exercise involving the fitting of the envelope initial conditions to provide a periodic envelope.

We then remarked on the more general approach to the problem given by Sacherer [31] and Lapostolle [14]. By averaging the trajectory equation over the phase space distribution function of a beam, they obtained equations for the RMS radii of the beam in the two transverse planes. These equations are identical in form to the $\mathrm{K}-\mathrm{V}$ envelope equations, at least for a beam with a real space distribution having elliptical symmetry. We now continue with some aspects of space-charge dominated transport in particular.

\section{A.1 Space-Charge Dominated Transport}

Even in the limit of zero emittance, the lattice will transport only a certain limiting current for a given $\sigma_{0}$ and bore radius. In the smooth approximation, this current depends on $\sigma_{0}$ for a constant bore radius, $R_{Q}$, as

$$
\mathrm{I}_{\mathrm{SA} .}=\frac{\pi \epsilon_{0} m v_{*}^{3}}{2 q}\left(\frac{R_{Q}}{L}\right)^{2} \sigma_{0}^{2}
$$


where we have written the equations in terms of a "period," $2 L$, over which the phase advance in the absence of current is given by $\sigma_{0}$. In this formula, $\sigma_{0}$ is in units of radians.

For an A.G. lattice, the current does not have this parabolic rise with $\sigma_{0}$ because of the envelope flutter required for the strong focusing. For a beam of zero emittance, the space charge forces cancel the external focusing and the beta function diverges. We have calculated the maximum beam radius for a beam with zero emittance in a thin lens FODO (see section 2.2.1) channel, using the envelope equations, Eqns. 2.5. With lenses of focal length $\pm f$ and separation $L$, we solved the envelope equations in tha approximation that the sum of the beam radii is nearly constant to obtain

$$
R_{\max }=\sqrt{\frac{q I}{2 \pi \varepsilon_{0} m v_{s}^{3}}} \frac{L}{2} \frac{2+\sin \left(\sigma_{0} / 2\right)}{\sin \left(\sigma_{0} / 2\right)}
$$

where $\sin \left(\sigma_{0} / 2\right)=L / 2 f$. We now obtain

$$
\mathrm{I}_{\text {A.G. }}=\frac{\pi \epsilon_{0} m v_{x}^{3}}{2 g}\left(\frac{R_{Q}}{\bar{L}}\right)^{2}\left(\frac{2 \sin \left(\sigma_{0} / 2\right)}{1+\frac{1}{2} \sin \left(\sigma_{0} / 2\right)}\right)^{2}
$$

This equation is written so that the leading factors are of the same form as for Eqn. A.1. The dependence on $\sigma_{0}$ of Eqn. A.2 reduces to that of Eqn. A.1 in the limit of low $\sigma_{0}$.

In the absence of instabilities, the ideal beam current in this approximation would appear to peak at $\sigma_{0}=180^{\circ}$, with a value of 2.8 times the $\sigma_{0}=60^{\circ}$ value. The parameterization in terms of the sine function hides the fact that $L / 2 f$ can exceed unity. The above result is valid within the range of $L / 2 f \leq 2$, rather than the zero-current limitation of $L / 2 f \leq 1$, but the same single-particle resonance with the focusing would require $\sigma_{0}<180^{\circ}$ even in the absence of collective instability of the beam. This is in marked contrast 
to the acceptance as a function of lattice strength for emittance-dominated beams. The limitations of alignment would require that this limit not be appraached very closely, even in the absence of collective instability. However, even at $\sigma_{0}=80^{\circ}$, the current is 1.5 times that for the same lattice at $\sigma_{\mathrm{o}}=60^{\circ}$. In a regime not limited by attainable focusing field gradients, much could be gained by increasing $\sigma_{0}$ to somewhat above $60^{\circ}$. This provides good reason for at empting the strongest focusing possible, and carefully probing the instablility limits of high-current beam transport.

\section{A.2 Useful Approximate Calculations}

In the process of this work, we found it simple and accurate to model the lenses as hard-edge quadrupoles, accounting for the space-charge forces in a smooth approximation correction to the focusing of the individual quadrupoles. We fit the occupancy factor of the quadrupoles, $\eta$, where

$$
\eta \equiv \frac{L_{G}}{L}
$$

$L_{Q}$ is the actual length of the lenses, and $2 L$ is the period of the focusing, to the single-particle pisase advance calculated numerically as a function of voltage. We found that $\eta=0.593$ gave very good agreement with the model $\sigma_{0}$ as a function of $V_{Q}$ from the ideal lattice calculation without space-charge, as shown in Table A.1.

Rather than solving the envelope equations numerically each time we needed an estimate for the matched beam size or for $\sigma$ for a particular set of beam parameters, we found that we could replace the space-charge forces in a simple way and replace the envelope equation integration by a transfer matrix calculation. In place of the space-charge forces, we calculated the 


\begin{tabular}{|c|c|c|c|c|c|c|}
\hline $\begin{array}{c}\sigma_{0} \\
(\mathrm{EE})\end{array}$ & $\begin{array}{c}\sigma_{0} \\
(\mathrm{SSC})\end{array}$ & $\epsilon_{\mathrm{unn}} / Q$ & $\begin{array}{l}R_{\max } \\
(\mathrm{EE})\end{array}$ & $\begin{array}{c}R_{\max } \\
(\mathrm{SSC})\end{array}$ & $(\stackrel{\sigma}{\mathrm{EE}})$ & (SSC) \\
\hline $59^{\circ}$ & $59^{\circ}$ & 0.0185 & 19.0 & 20.1 & $8^{\circ}$ & $7^{\circ}$ \\
\hline $78^{\circ}$ & $78^{\circ}$ & 0.0177 & 15.9 & 16.7 & $12^{\circ}$ & $11^{\circ}$ \\
\hline $\mathbf{8 3}^{\circ}$ & $83^{\circ}$ & 0.0215 & 15.5 & 16.3 & $17^{\circ}$ & $15^{\circ}$ \\
\hline $88^{\circ}$ & $88^{\circ}$ & 0.0286 & 14.8 & 15.5 & $24^{\circ}$ & $21^{\circ}$ \\
\hline $91^{\circ}$ & $91^{\circ}$ & 0.0242 & 13.7 & 14.5 & $22^{\circ}$ & $19^{\circ}$ \\
\hline $94^{\circ}$ & $94^{\circ}$ & 0.0324 & 12.5 & 13.0 & $29^{\circ}$ & $26^{\circ}$ \\
\hline $97^{\circ}$ & $97^{\circ}$ & 0.0800 & 13.1 & 13.6 & $57^{\circ}$ & $52^{\circ}$ \\
\hline $102^{\circ}$ & $102^{\circ}$ & 0.100 & 11.1 & 11.3 & $67^{\circ}$ & $63^{\circ}$ \\
\hline $116^{\circ}$ & $116^{\circ}$ & 0.172 & 8.9 & 9.0 & $93^{\circ}$ & $88^{\circ}$ \\
\hline $124^{\circ}$ & $124^{\circ}$ & 0.151 & 13.3 & 13.1 & $99^{\circ}$ & $92^{\circ}$ \\
\hline $135^{\circ}$ & $134^{\circ}$ & 0.156 & 9.8 & 9.6 & $108^{\circ}$ & $101^{\circ}$ \\
\hline $144^{\circ}$ & $142^{\circ}$ & 0.145 & 11.6 & 11.3 & $114^{\circ}$ & $104^{\circ}$ \\
\hline
\end{tabular}

Table A.1: Smooth space charge model compared with envelope equation integration. We have included the relative values of the emittance and current through the quantities appearing in the envelope equation, as the ratio of the unnormalized emittance, $\epsilon_{\text {unn }}$, to the generalized perveance, $Q$. Units for the various quantities are $\%$ period for the tunes, $\pi$ meter radian for $\epsilon_{\text {unn }} / Q$, and $\mathrm{mm}$ for radius. The notation (EE) represents quantities calculated using the envelope equations, while (SSC) denotes the same physical quantities estimated by averaging the space-charge forces along the lattice. 
equivalent defocusing lens strength for the beam, $K_{B}$, as

$$
K_{\mathrm{B}}=\frac{Q}{2 R_{0}^{2}}=\frac{q I}{\pi \epsilon_{0} m v_{s}^{3} R_{0}^{2}}
$$

taking the radius $R_{0}$ between quadrupoles as the appropriate size for the beam to calculate the average space-charge defocusing field. We calculated the quadrupole strength, $K_{Q}$, as

$$
K_{Q}=\frac{4 q V_{Q}}{m v_{s}^{2} R_{Q}^{2}}
$$

and used net focusing and defocusing strengths of $K_{+}$and $K_{-}$, respectively, of

$$
\begin{aligned}
& K_{+}=K_{Q}-K_{B} \\
& K_{-}=K_{Q}+K_{B} .
\end{aligned}
$$

The drift portion of the lattice is then treated as a defocusing lens.

After calculating the transfer matrix through one period, beginning at the midplane between quadrupoles and with a particular $K_{B}$, we used the relationship $R_{0}^{2}=\beta \epsilon$ to calculate the ratio $\epsilon / I$. The half-trace of the transfer matrix including space-charge forces gives the corresponding $\sigma$. We found very good agreement between this model and the envelope integration model using the $\mathrm{K}-\mathrm{V}$ equations. The thin lens lattice is solvable in simpie closed form for $\epsilon=0$, with the result for the transportable current given above in Eqn. A.2. The result for the maximum current through a given bore in the $\epsilon \rightarrow 0$ limit for the smooth space-charge model is

$$
I_{\text {s.s.c. }}=\frac{\pi \epsilon_{0} m v_{x}^{3}}{2 q}\left(\frac{R_{Q}}{L}\right)^{2}\left[\frac{2 \sin \left(\sigma_{0} / 2\right)}{\exp \left[\frac{1}{2} \sin \left(\sigma_{0} / 2\right)\right]}\right]^{2}
$$


as a function of $\sigma_{0}$, where we have written the equation in the same form as Eqns. A.1 and A.2. For small values of $\sin \left(\sigma_{0} / 2\right)$, Eqn. A.3 reduces to the envelope equation result. Even at extreme lattice strengths this model agrees well with results obtained from the envelope equations. Even for $\sigma_{0}$ as high as $180^{\circ}$, the ratio of limiting currents from the approximate solution of the envelope quations above and this smooth space charge calculation is

$$
\left.\frac{I_{\text {S.S.C. }}}{I_{\text {A.G. }}}\right|_{180^{\circ}} \simeq 0.83
$$

and for $v_{0}=90^{\circ}$

$$
\left.\frac{I_{\text {S.S.C. }}}{I_{\text {A.G. }}}\right|_{900} \simeq 0.90
$$

The ratio of the currents is about 0.95 for a $60^{\circ}$ lattice. These two approximations to the solution of the envelope equations converge to the exact solution in the limit of small lattice strength. Because the current is proportional to the product of the two beam dimensions, the actual beam radius is less in error than the above numbers for relative current would suggest. The S.S.C. form is only a few percent in error for calculating maximum envelope radius (and $\sigma$ ) as a function of $\epsilon, I$, and $\sigma_{0}$ over a very wide range of lattice strength, particularly if the current is a significant factor in the transport. We implemented this procedure on a programmable hand calculator for quick estimates of $\sigma, R_{+}$, and $R_{0}$ for the SBTE, as well as for stability estimates for $\mathrm{Cs}^{+2}$ ions in the space charge field of the $\mathrm{Cs}^{+}$beam. 


\section{Appendix B: Faraday Cups}

The charge collecting diagnostics must measure the total ion beam current with miniraal error. In addition, emittance measurements require the precise relative measurement of currents down into the sub-microampere range. For this purpose, a large secondary emission gain would be very useful if the gain were uniform. In this appendix we discuss the methods we used to attain these goals and the uncertainties of the measurements. We chose to measure the total beam current by direct ion collection, and to use the emittance diagnostic cups in the secondary emission (SE) mode.

\section{B.1 Secondary Electron Yields}

Relative measurement of small beam currents by diagnostics using secondary particle emission as an amplifying mechanism is very easy. Homogeneous plate materials are not expected to show large variation in secondary electron emission gain over their surfaces. In our measurements, we have seen only variations due to bias fields and angle of primary particle impact. The measured secondary electron yield on stainless steel sheet stock for normally incident $\mathrm{Cs}^{+}$ions in the $120-160 \mathrm{keV}$ range is 12 , depending only weakly on applied bias field up to a few $\mathrm{kV} / \mathrm{cm}$ strength. This is the result both for the full beam and for very highly attenuated beams during emittance scans. The ave:age gain across the diameter of 0.020 in tungsten wires has been measured to be about the same for our multiwire profile monitors, again about a factor of 12 .

\section{B.2 Slit Cups}

The slit cups (shown in Fig. 3.12) are used only for measuring the emittance or current profile of a beam. This function is served very well by the reverse biased secondary emission mode. The cup bias response in this mode 
is flat from 100 to $500 \mathrm{~V}$. We used $\pm 300 \mathrm{~V}$ biases for G2 and C, respectively. In the slit cups, the "grid" G1 is the slit itself and is expicitly grounded. The ratio of back- to forward-biased current signals is about 10, a somewhat lower ratio than found for the other cups, but we do not consider the difference significant.

\section{B.3 Absolute Current Measurement}

\section{B.3.1 Shallow Faraday Cups}

When we attempt to make absolute current measurements with a shallow Faraday cup, we must introduce at least one grid to suppress secondary ernission from the collector. We use two grids, each of at most $98 \%$ transparency. We thus expect to have electron currents of as much as $40 \%$ of the incoming beam current flowing neas the grids and must control the electrons very well in order to measure the ion current accurately.

We have labeled the electrodes as in Fig. 3.9. When grounding $G 1$ and biasing $G 2$ negative and $C$ positive, the cup current did not crisply saturate. The cup current continued to drop very slowly as the potential difference $C-G 2$ was raised. We found a somewhat crisper saturation for the reverse bias, secondary emission mode, but the current continued to rise very slowly as the potential difference $C-G 2$ was lowered.

Secondary electrons from the positively biases collector are energetically unable to escape to grounded surfaces, and the negative $G 2$ bias prevents the - from being drawn out by the beam potential of about +600 volts. Some $e^{-}$from $G 2$ may be collected at $C$. With the grid $G 1$ still at ground potential we found a transient negative current signal at the tail of the beam pulse. The time development may be seen in Fig. B.1. These effects are shown in Fig. B.1. The negative transient signal as a function of pulse duration is quite well correlated with the deviation of the current pulse shape from a square 


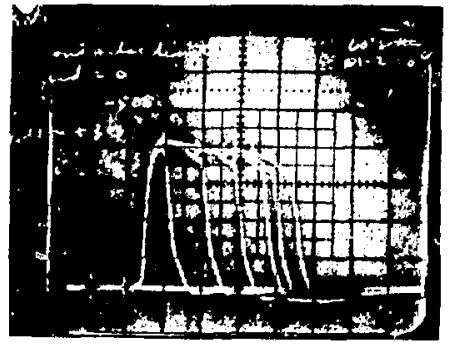

(a)

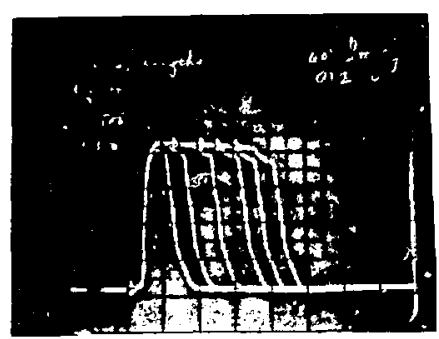

(b)

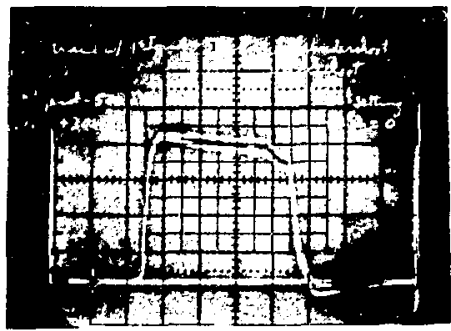

(c)

XBB $864-2706$

Figure B.1: Current response of the SFC. With the first grid grounded, the head and tail of the current pulse showed a saturation in SFC response at different biases (not shown). After we had chosen a bias configuration which gave a reasonable saturation over the length of the pulse, we noted an undershoot of the measured current at the end of the pulse. By overlaying pulses of various length (a), we found that the envelope of the undershoot closely approximated the deviation of the SFC response from the flat current vs. time we had expected. While keeping the same positive bias on the collector, we found that we could suppress the undershoot by biasing the first grid, $G 1$, to a higher positive potential than we biased the collector (b). A positive bias on $G 1$ had little effect for values lower than the collector potential, but with the biases $G 1=+500$ and $G 2=-500$, the current showed no variation $c$.er approximately the range $100<C<400$, and the undershoot was almost totally eliminated. We hopted to use $G 1=+500$, $G 2=-500$, and $C=+300$. The comparison between the initial and final bial configurations is shown in (c). 
pulse. However, with bias felds of the order of a $1 \mathrm{kV} / \mathrm{cm}$, the $\sim 1 \mu \mathrm{sec}$ decay time scale of the undershoot indicates that it is an ion effect rather than an electron effect. We must assume that there is a small secondary yield of positive ions, which are flowing from the collector to the grids.

We found that biasing $G 1$ to $+500 \mathrm{~V}$ essentially eliminated the cup undershoot. Any secondary positive ions will be unable to penetrate the $G 1$ bias and will be reflected preferentially to the collector. An additional benefit is the enhancement of the local electric field at $G 2$ on the upstream side, minimizing entry of secondary electrons into the $G 2-C$ region. We thus chose to operate the SFC's with a collector bias of +300 volts, biasing the grids G1 and G2, respectively, to \pm 500 volts.

The shallow cups were fabricated at various times, with grids of differing transparency, and so had different calibration factors. The comparison can be seen in Fig. 5.3, showing the direct response of all the cups to a beam with no known $\mathrm{Cs}^{+}$component loss exceeding about $2 \%$ over the lattice length.

\section{B.3.2 Deep Faraday Cups}

The DFC (deep Faraday cup) was designed by Dr. C. Kim using Hermannsfeldt's EGUN [34] program to calculate the axial potentials, including the beam space-charge. The resulting design is shown in Fig. 3.10. The collector and repeller biases may be raised to $\pm 5 \mathrm{kV}$. Operation with $C=+2 \mathrm{kV}$ and $R=-2 \mathrm{kV}$ will result in a potential minimum along the axis of -200 volts even in the presence of $15 \mathrm{~mA}$ of $120 \mathrm{keV} \mathrm{Cs}^{+}$. This provision was made because "electrons are everywhere," and we wanted to avoid any trouble with primary electrons.

The DFC bies curve saturates crisply at both polarities, the bias ring preventiug eiectrons from entering (negative bias) or leaving (positive bias) in each case, as shown in Fig. B.2. The ratio of the currents in the two bias 


\section{Deep Faraday cup bias curves for various bias conditions}

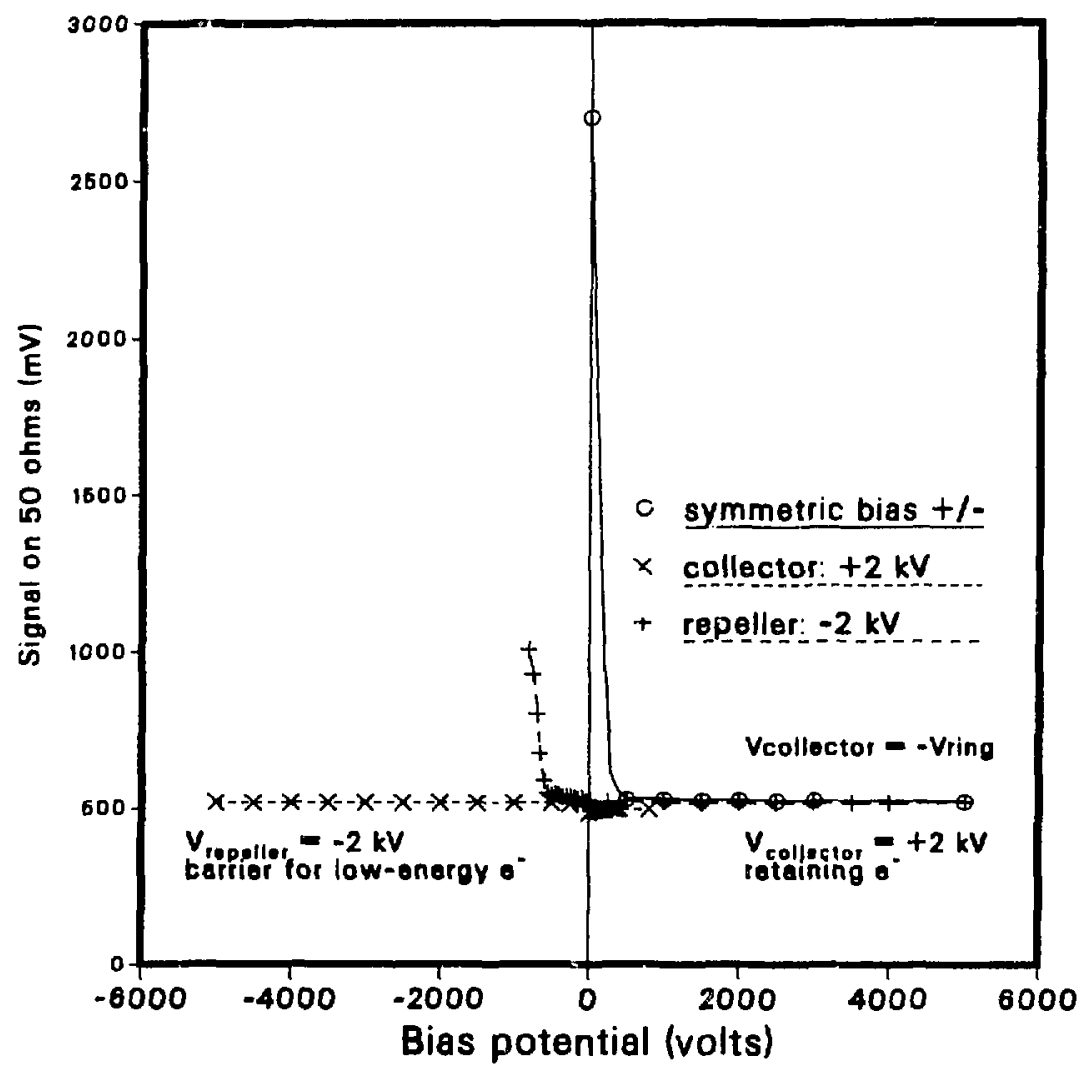

XBL 865-1839

Figure B.2: Bias curves for the DFC. With biases for the collector and the repeller ring differing only in polarity, the DFC response saturated cleanly in both the secondary electron retention and secondary emission modes. The ion current saturated at $\pm 500 \mathrm{~V}$, respectively, for the collector and repeller, but we operated at the design bias of $\pm 2 \mathrm{kV}$. This maintains a negative potential across the central plane of the repeller ring to prevent any possibile entry of low-energy electrons. 
modes is again about 12 . The absolute current calibration is thus subject to error primarily by secondary positive ion emission. The bore clearance is sufficient to avoid all problems of primary ion impact on the repeller ring. The major compromise lies in the inherently poor spatial resolution that arises from the capacitive coupling of the cup to the beam it is measuring. The slow beam results in a risetime for current perturbations of only 150-200 $\mathrm{ns}$ because the coupling of a given beam ion to the cup takes place over a 2-3 in distance centered at the 1 in gap separating the collector and repeller.

\section{B.3.3 Secondary Ion Effects}

We estimated the magnitude of the error in current measurement caused by the secondary ions by using the DFC near the source, where the current pulse drops sharply at the pulse end. Typical data are visible in Fig. 5.3. The undershoot we saw in the SFC traces is visible for short fall-times of the current pulse. The DFC after Q82 showed no undershoot because the long pulse tail masked any negative current transient. The magnitude of the ion error signal is about $4 \%$ of the total current. Some of the ions are probably collected by the repeller ring, but with too low an energy to result in significant secondary electron emission. Because we do not believe that the true beam current is less than the indicated value, and because the secondary ion emission (assumed to be the dominant error mechanism, lowering the mleasured current below the true value) appears to affect the current reading by no more than about $4 \%$, we quote our uncertainty in the value of the beam current as $I=I_{\text {masured }}^{+4 \%}$. 
Appendix C: Emittance Increase due to Grids in the Beam Path

In the SBTE, we use grids at the source for two purposes. The primary purpose is to terminate the accelerating field without grossly defocusing the beam. We also use additional, externally biased grids to increase the beam emittance in a controlled fashion. We will discuss the performance and mechanism of operation in this appendix.

Fine-grained spatial variation of a transverse electric field at the grid scatters the beam particles out of their former phase space positions. We will use $4 \epsilon_{\mathrm{RMS}}$ to parameterize the beam emittance, given by

$$
4 \epsilon_{x} \mathrm{RMS}=4\left\langle(x-\bar{x})^{2}\right)^{\frac{1}{2}}\left\langle\left(x^{\prime}-\overline{x^{\prime}}\right)^{2}\right\rangle^{\frac{1}{2}}=2 \tilde{x} \times 2 \tilde{\theta}_{x} .
$$

We use the quantity $2 \tilde{x}$ for the beam radius, and it is convenient to use $2 \tilde{\theta}$ as the angular spread parameter.

In the SBTE, the beam particle energy is an order of magnitude larger than the potential applied to the grids, and the impulse approximation is sufficient to calculate the velocity space perturbation of the beam. The intrinsic RMS angular spread of the beam adds in quadrature to the RMS grid perturbation angle, so that the grid perturbation adds a term in quadrature to the intrinsic source emittance.

Because the measurements reported in this Appendix were not the central concern of the program, and were compiled from scattered results throughout the time span of the experiment, some of the data are from operation with the original, $160 \mathrm{keV}$ injector configuration, and some are from the later configuration operated with a particle energy of $120 \mathrm{keV}$. As a result, some conceptually related calculations are reported for different particle energies. 


\section{C.1 Mechanism}

The two sources of electric field acting upon the grid are the beam self-field and externally applied fields. We will first examine the effect of a single grid terminating the acceleration field of the injector, and then of an externally biased grid array.

We will model a parallel-wire grid as an array of slots, each as in Fig. C.1. In the impulse approximation, a particle transiting the slit experiences a lens action with an equivalent focal length

$$
f_{\mathrm{S}}=\frac{2 T}{\Delta E_{\|}}
$$

where $T$ is the particle kinetic energy in $\mathrm{eV}$ and $\Delta E_{\|}$is the change in longitudinal field.

As shorthand, we define a parameter $\alpha$ by

$$
\alpha \equiv \frac{\Delta E_{\|}}{4 T}
$$

For a parallel-wire grid, calculatir.g the perturbation in the plane transverse to the grid, we have

$$
\Delta \theta_{\mathrm{l}}=2 \alpha r,
$$

where $r$ is the distance the particle passes from the center of the gap between wires. Thus, for an aperture width of $2 R$, we have

$$
\left\langle\theta_{\mathrm{t}}^{2}\right\rangle=\frac{\int_{0}^{R}(2 \alpha r)^{2} d r}{\int_{0}^{R} d r}=\frac{4}{3}(\alpha R)^{2}
$$

in the plane perpendicular to the slit. When the grid is oriented at $45^{\circ}$ with respect to the $x$ and $y$ planes, the result is

$$
2 \tilde{\theta}_{x}=2 \sqrt{\frac{2}{3}} \alpha R \simeq 1.63 \alpha R
$$




\section{Electric field lines near grid wires}

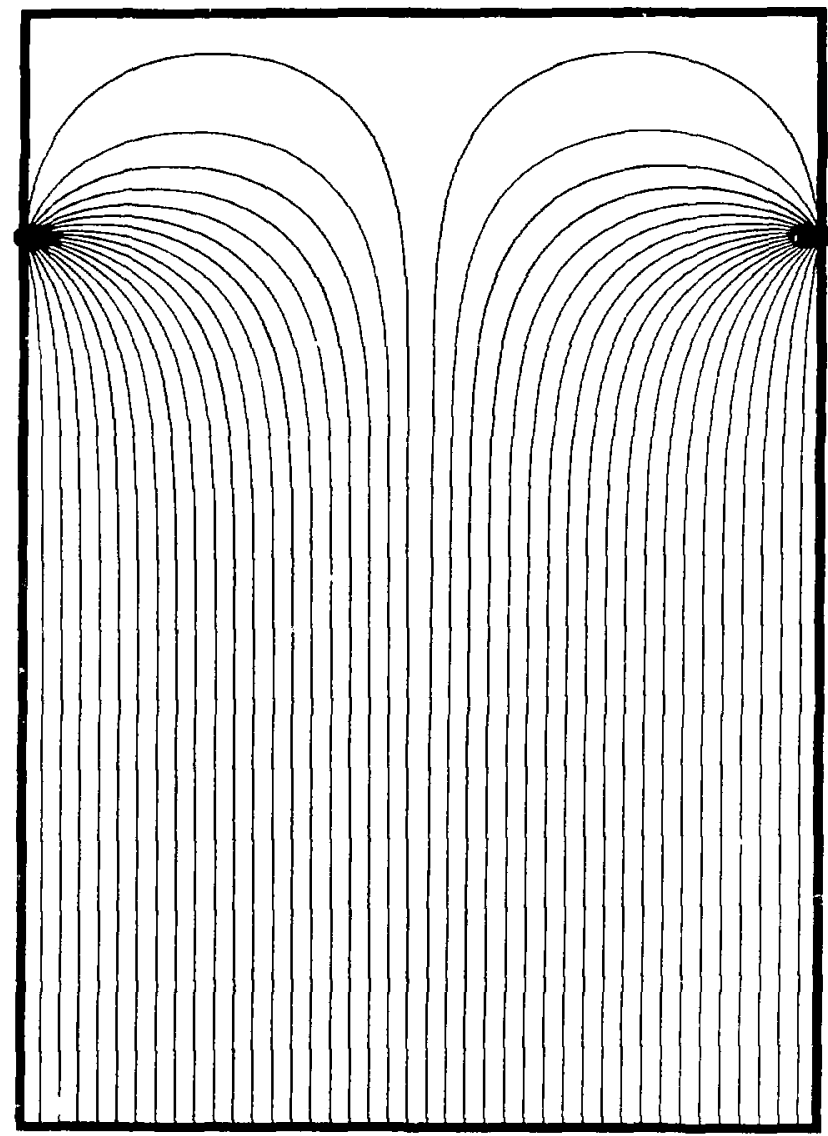

XBL $865-1759$

Figure C.1: We show field lines in one cell of a parallel-wire grid, with wires at either side of the figure, perpendicular to the plane of the page. The boundary conditions include a uniform field in the vertical direction at the bottom of the figure, with a feld-free region far above the grid. The electric field focuses the particles transiting the region, with a focal length dependent on the difference in the electric field component along the direction of travel of the particles. 


\begin{tabular}{c|c|c|} 
Hex grid & $45^{\circ}$ line grid & $90^{\circ}$ line grid \\
\hline$\alpha R$ & $1.63 \alpha R$ & $2.31 \alpha R$ \\
\hline
\end{tabular}

Table C.1: Variation of the grid perturbation of the emittance with grid structure. The quantity $\alpha$ depends on the difference in longitudinal electric field on the two sides of the grid, and $R$ is the radius of a circular aperture or the half-spacing of wires making up the grid.

A circular aperture under the same conditions gives a focal les zth

$$
f_{\mathrm{C}}=\frac{4 T}{\Delta \Sigma_{\|}}
$$

in each transverse plane. We have used both parallel-wire grids and metal honeycomb grids, which we will model as arrays of slit and circular apsrtures, respectively. The radial angular impulse $\Delta \theta_{r}$ for a particle transiting a circular aperture is

$$
\Delta \theta_{\mathrm{r}}=\alpha r
$$

as a function of the distance $r$ from the center of the aperture, so we may calculate $\left\langle\theta_{r}^{2}\right\rangle$ as

$$
\left\langle\theta_{r}^{2}\right\rangle=\frac{2 \pi \int_{0}^{R}(\alpha r)^{2} r d r}{2 \pi \int_{0}^{R} r d r}=\frac{(\alpha R)^{2}}{2}
$$

Because the perturbations in the two transverse planes are equal, and because $\left\langle\theta_{r}^{2}\right\rangle=\left\langle\theta_{x}^{2}+\theta_{y}^{2}\right\rangle=\left\langle\theta_{x}^{2}\right\rangle+\left\langle\theta_{y}^{2}\right\rangle$, we have

$$
2 \tilde{\theta}_{x}=\alpha R
$$

The results are summarized in the Table C.1. 
C.2 Termination of the Accelerating Field of the Injector

In the the low-perveance configuration of the injector, we did not grade the aperture plates exactly as for a planar Pierce diode. The applied field was peaked at the center of the gun to provide some additional focusing, so the field at the exit grid was somewhat below the planar diode value $4 V_{\text {gun }} / 3 L_{\text {gun }}$, which has the value $2.3 \mathrm{MV} / \mathrm{m}$. We will use $\Delta E_{\|}=2 \mathrm{MV} / \mathrm{m}$, so that $\alpha=3.125$ for $V_{\text {gun }}=160 \mathrm{kV}$.

In this configuration, the beam charge density $\rho$ at the grid was $90 \mu C / \mathrm{m}^{3}$. We will compare the relative effect from the segmented beam space-charge by calculating $\Delta \theta$ as a function of distance from the center of a slab, due to the self-field of the individual slab over a distance in $z$ of twice the wire separation (the wire separation is $2 R$ ).

$$
\Delta \theta(r)=\frac{q E_{\perp}}{m v_{x}} \frac{4 R}{v_{z}}=\frac{2 \rho R}{\epsilon_{0} V_{\text {gun }}} r
$$

so that

$$
f=\frac{r}{\Delta \theta(r)}=\frac{2 V_{\mathrm{gun}}}{4 R \frac{\rho}{\epsilon_{0}}}
$$

For a grid spacing of 0.062 in, the quantity $4 R \rho / \epsilon_{0}$ is about $30 \mathrm{kV} /$ meter, negligible with respect to the accelerating gradient of $2 \mathrm{MV} /$ meter.

We estimate the intrinsic gun emittance from measurements with a $9 \mathrm{~mm}$ beam radius and relativistic $\beta_{r}$ of $C . .^{*} 6$, using

$$
\epsilon_{I}^{2}=\epsilon_{T}^{2}-\epsilon_{G}^{2}
$$

where $\epsilon_{\mathrm{T}}$ is the net emittance after the grid and

$$
\epsilon_{\mathrm{G}}=R_{\mathrm{Bearm}}\left(2 \tilde{\theta}_{x}\right) \beta-\gamma
$$




\begin{tabular}{l|c|c|} 
& \multicolumn{1}{c}{ Line grid } & Hex grid \\
\cline { 3 - 4 }$\epsilon_{\mathrm{T}}$ & $(1.15-1.25) \times 10^{-7} \pi$ & $(1.3-1.4) \times 10^{-7} \pi$ \\
\cline { 3 - 3 }$\epsilon_{\mathrm{G}}$ & $0.58 \times 10^{-7} \pi$ & $0.73 \times 10^{-7} \pi$ \\
\cline { 3 - 4 }$\epsilon_{\mathrm{I}}$ & $(0.99-1.11) \times 10^{-7} \pi$ & $(1.07-1.19) \times 10^{-7} \pi$ \\
\cline { 2 - 3 } &
\end{tabular}

Table C.2: Intrinsic emittance of gun before grid passage

\begin{tabular}{|c|c|c|}
\multicolumn{1}{c}{$\begin{array}{c}\text { Normalized Emittance } \\
\left(10^{-7} \pi \text { meter radian }\right)\end{array}$} \\
\multicolumn{1}{c|}{ Grid geometry } & \multicolumn{1}{c|}{ measured } & calculated $\left(\epsilon_{1}=1.1\right)$ \\
\hline 0.063 in wire & 1.3 & 1.44 \\
\hline 0.050 in ribbon & 1.4 & 1.33 \\
\hline 0.125 in hex & 1.75 & 1.6 \\
\hline 0.0625 in hex & 1.3 & 1.25 \\
\hline
\end{tabular}

Table C.3: Relative effect of various terminating grids vs. calculation The results are shown in Table C.2.

We compare four different grid geometries for the $120 \mathrm{kV}$ gun, one at a time. We used wires on 0.062 in centers, ribbon naterial on 0.050 in centers, and 0.125 in and 0.062 in hex material, with $\Delta E_{\|}=2 \mathrm{MV} / \mathrm{m}, R_{\mathrm{B}}=12 \mathrm{~mm}$, and relativistic $\beta_{r}=0.0014$. Table $C .3$ summarizes the results.

\section{C.3 Effect of Multiple Biased Grids}

$N, w$ we calculate the effect of our multiple emittance-spoiling grids, for which the effective spacing is $4 \mathrm{~mm}$. We have

$$
\left(2 \tilde{\theta}_{\mathrm{G}}\right)^{2}=\left(R_{1} \alpha_{1}\right)^{2}+\left(R_{2} \alpha_{2}\right)^{2}+\left(R_{3} \alpha_{3}\right)^{2}
$$


For grids of uniform 0.125 in cell size, with relativistic $\beta_{r}=0.0016$ and $R_{B}=9$ mm, we obtain for the normalized emittance

$$
\epsilon_{G}=0.37 \sqrt{4+V+\frac{3}{8} V^{2}} \times 10^{-7} \pi \text { meter radian, }
$$

where $V$ is the voltage applied to the central grid in $\mathrm{kV}$. For the $120 \mathrm{kV}$ configuration, we had $\beta_{r}=0.0014$, and the cell size of the second grid was 0.062 in. Numerically, with $R_{\mathrm{B}}=12 \mathrm{~mm}$, the normalized emittance is

$$
\epsilon_{G}=0.56 \sqrt{4+V+\frac{3}{16} V^{2}} \times 10^{-7} \pi \text { meter radian }
$$

The results are shown along with measurements in Fig. C.2. The agreement is very reasonable, although the grid spacing for the $120 \mathrm{kV}$ configuration may be somewhat different from that for the $160 \mathrm{kV}$ case. 
as a Function of Applied Potential,

Compared with Measurement

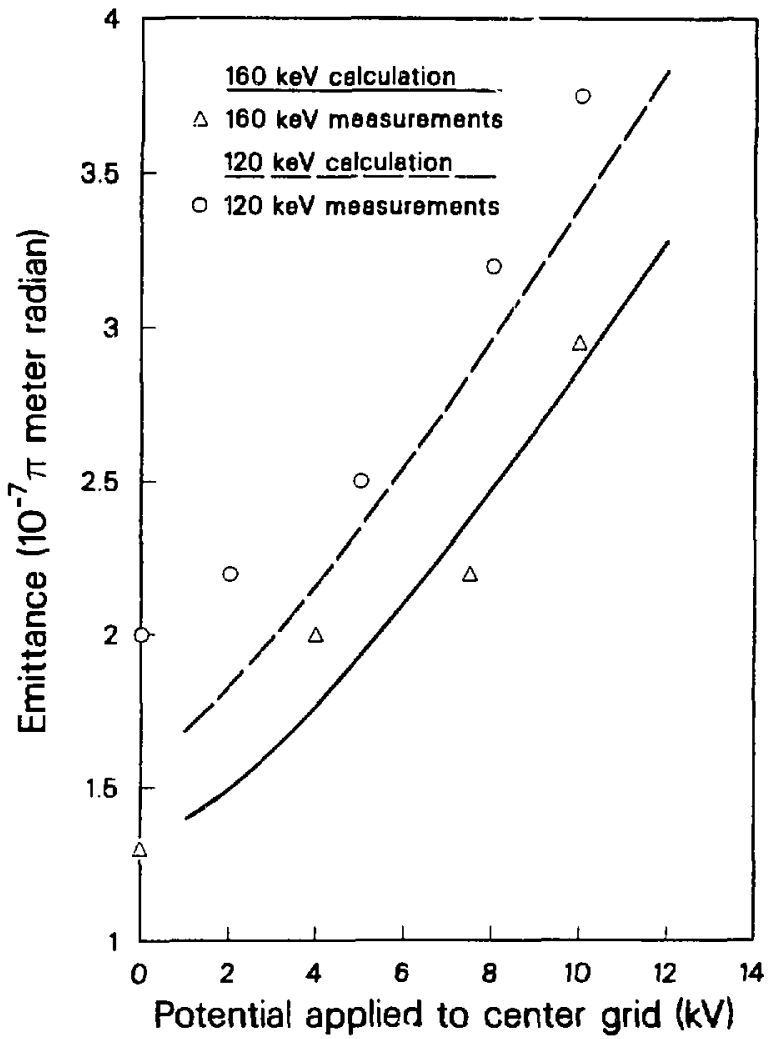

XCG $854-7170$

Figure C.2: Grid spoiling of the emittance compared to calculated values. 


\section{Appendix D: Quadrupole Design}

The SBTE was designed to make a fundamental experimental test of collective beam dynamics in a linear focusing lattice. Hence it is important that the actual focusing be linear insofar as possible. To enable detailed, high-resolution diagnostic capability, and to maximize collective effects, both the bore radius and beam current must be held as high as possible, consistent with maintaining focusing linearity. To satisfy these requirements, Dr. L. J. Laslett designed an electrode shape which provided highly linear focusing fields [50], while at the same time providing for relative ease of manufacture. The resulting pole-tip design is shown in Fig. D.1.

The fields of periodic arrays with various electrode shapes were examined using a 3-dimensional relaxation calculation. The field corresponding to the Gnal electrode design was decomposed as a Fourier-Bessel series, truncated to the first seven nonzero terms in $\cos (2 \theta)[50]$, as

$$
V(r, \phi, z)=\left[\sum_{m=1}^{\tau} A_{m} I_{2}\left(\frac{(2 m-1) \pi r}{l}\right) \sin \left(\frac{(2 m-1) \pi z}{l}\right)\right] \cos (2 \phi) .
$$

Most of the terms missing from the general form of the Fourier-Bessel expansion are identically zero due to the symmetry of the lattice and the choice of coördinate o:igin. Setting $\phi=0$ in one of the quadrupole focusing planes suppresses the $\sin (n \phi)$ terms; the potential is finite at $r=0$, so the coefficients of the functions $K_{n}$ are all zero; and choosing the z-origin so that $z=0$ at the midplane between quadrupoles ensures that the $\cos \left(\frac{m \pi z}{l}\right)$ and $\sin \left(\frac{2 m \pi z}{l}\right)$ coefficients are all zero. The values of $n$ allowed by the lattice symmetry for the azimuthal dependence are of the form $4 k+2$, and we kept only the quadrupole, or $\cos (2 \phi)$ terms for the field expansion used in later particle tracking and envelope integration programs. We list the coefficients for the 


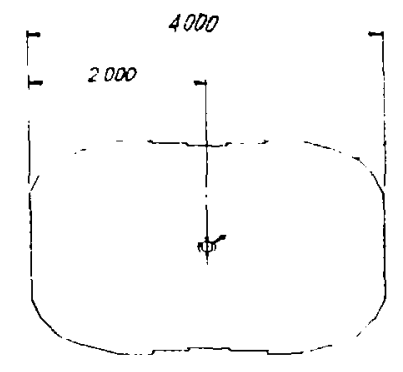

$121(1590)$ DR/LL, 875 $010 D P$ 10-32 UNF TAP, $688 \pm .010$ DP
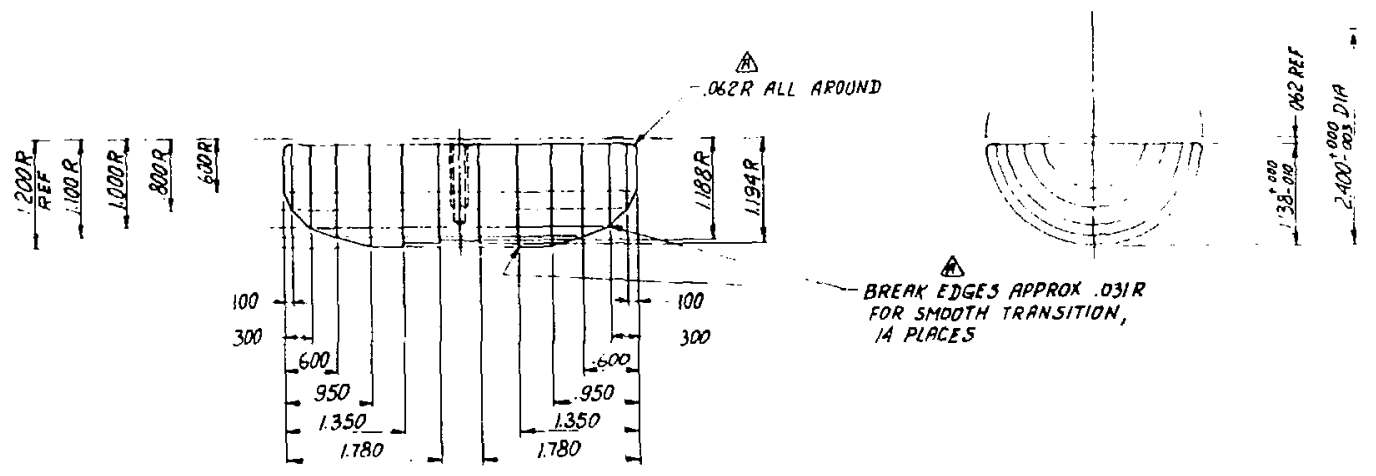

- BREAT EDGES APPROX .03/R BREAT EDGES APPROX .OJIR
GOR SMOOTH TRANSITION. 14 PLACES

Figure D.1: SBTE electrostatic quadrupole dimensions. 


\begin{tabular}{|c|c|c|}
\multicolumn{1}{c}{$m$} & \multicolumn{1}{c}{$A_{m}$} & $A_{m} I_{2}\left(\frac{(2 m-1) \pi}{B}\right)$ \\
\hline 1 & +29.92578 & +1.049172 \\
\hline 2 & -.0521235 & -.01964735 \\
\hline 3 & -.0561377 & -.08217125 \\
\hline 4 & +.0007866 & +.03580476 \\
\hline 5 & +.0004084 & $+.00 u s 7351$ \\
\hline 6 & -.0001266 & -.00467973 \\
\hline 7 & $+.00005 \% 3$ & +.00547446 \\
\hline
\end{tabular}

Table D.1: Coefficients used in field expansion

resulting series in Table D.1. Because the Bessel function $\mathrm{I}_{2}$ may attain large values at the bore radius, the products of these coefficients with their Bessel function multipliers at that radius are also included. Note that near the bore radius, the contributions of the higher-order terms are not becoming rapidly smaller. Nearer the axis, however, the high-order Bessel functions drop much. more rapidly than the lowest-order nis, because for small $z$,

$$
\mathrm{I}_{n}(z) \rightarrow \frac{1}{n !}\left(\frac{z}{2}\right)^{n}
$$

The peak value of the series for the listed coefficients for a 1.0 in radius and $\phi=0$ is about 1.04, and occurs at a point on the electrode surface. The value here should be unity, indicating that the level of error in the expansion is about $4 \%$, at least at this large radius.

This field representation was used to calculate $\sigma_{0}$ for the periodic lattice as a function of $V_{Q}$ for various beam emittances. The beam calculations were carried out using the envelope equations for linear applied field by taking the local field at the beam edge, obtaining a value for the focusing gradient by dividing by the local beam radius. This gives an average focusing gradient over the beam diameter for use in the envelope equations, while including 
the field nonlinearities in a very approximate way to take into account the variation in the focusing strength with beam radius. The value of $\sigma_{0}$ was obtained by performing the integral

$$
\sigma_{0}=\int_{x_{0}}^{z_{0}+3 L} \frac{\epsilon d z}{R_{x}^{2}}
$$

along with the beam envelope calculation, where the initial conditions had been chosen so that the beam was matched. As an additional check on the accuracy of the field representation, a comparison was made with the neeld generated froro a relaxation calculation constrained to have $\cos (2 \theta)$ symmetry. The resulting Fourier-Bessel coefficients were used in the same way to calculate the lattice $\sigma_{0}$ and the agreement between the two calculations for beam envelope values for $\sigma_{0} \simeq 60^{\circ}$ was better than 1 part in $10^{5}$, giving support to the conclusion that Eqn. D.1 is an accurate description of the field within the periodic lattice of the SBTE.

Fig. D.2 shows $\left.E_{z}(x)\right|_{y=0}$ for several values of $z$ along a lattice period, and in Fig. D.3 we have plotted the values of $\sigma_{0}$ vs. $a_{\max }$ calculated for various input beam emittances with $I=0$. The residual lattice nonlinearity is apparent in the variation of $\sigma_{0}$ with $a_{\text {max }}$.

For the lattice fields alone, it is relatively simple to determine the linearity of the focusing. However, in the high space-charge beam transport cases we wanted to investigate, the beam self-field provides a large perturbation to the lens fields, and is expected to be a strong source of field nonlinearity that could far outweigh the residual lens nonlinearity.

In summary, the lattice of the SBTE, using the electrode shape designed by Dr. Laslett, provides a good approximation to in linear focusing lattice, evin with the extreme electrode length to bore radius ratio of 4 that was used. The short period length permitted a high average current density for 
Fields of the SBTE Quads as a Function of Radius at Various Z-positions

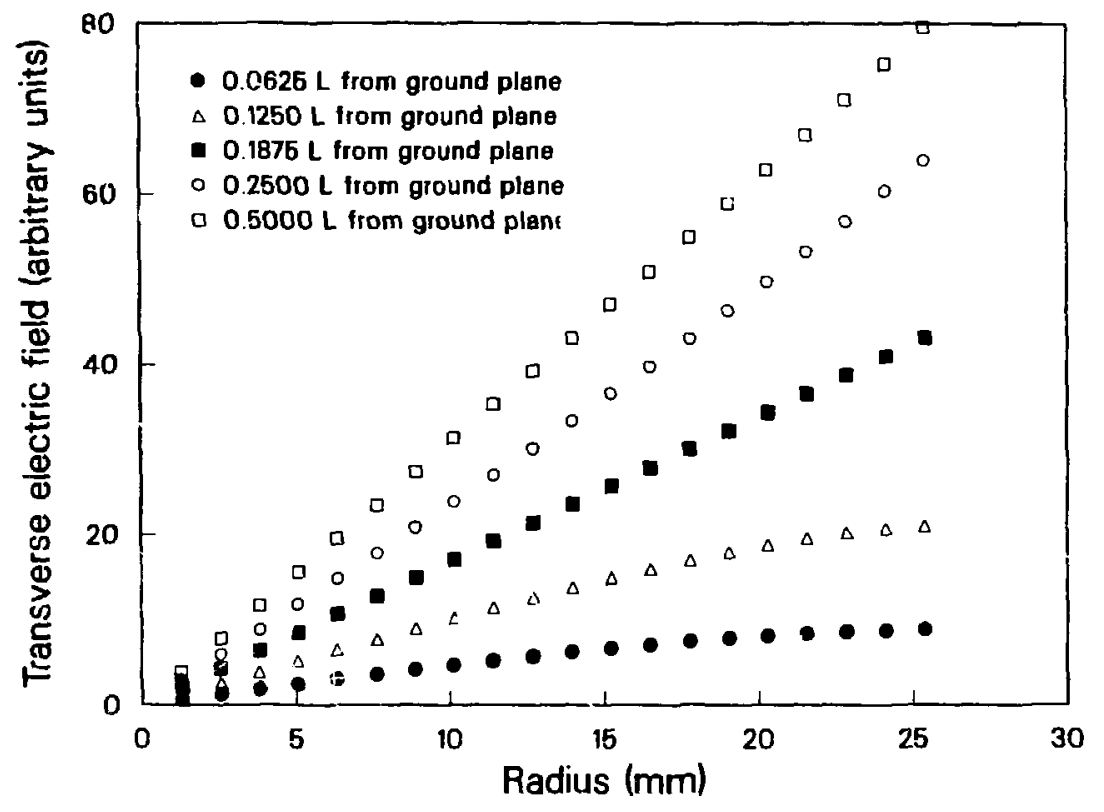

$x C G 864-7165$

Figure D.2: Transverse field lineàrity ri the SBTE periodic lattice. 


\section{Variation of $\sigma_{0}$ with Quadrupole Voltage}

at Various Oscillation Amplitudes

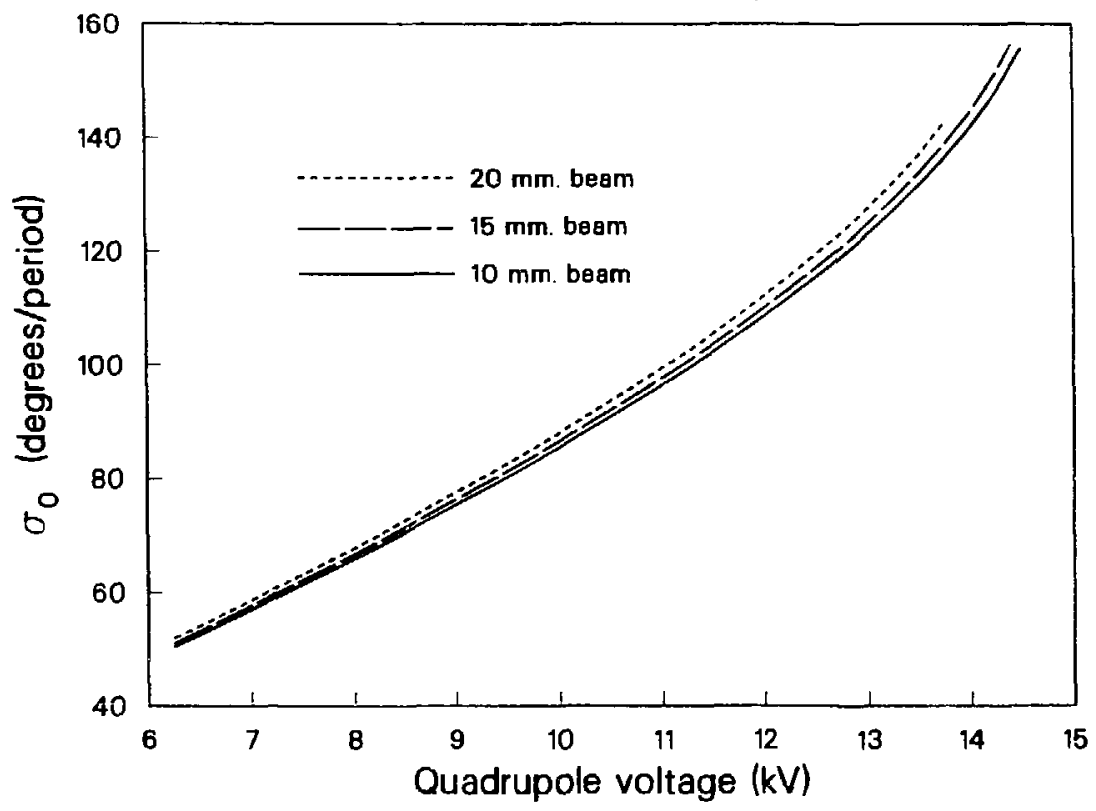

XCG $864-7171$

Figure D.3: Variation of $\sigma_{0}$ in the SBTE with particle oscillation amplitude. 
space-charge dominated beam transport. Also, both the lens separation of 2 in and the large bore radius allowed high resolution diagnostics. For example, the sample width of the slit diagnostics was 0.010 in $(0.25 \mathrm{~mm})$, compared to a typical beam diameter of $12 \mathrm{~mm}$. 


\section{Appendix E: Ion Sources}

We discuss the features of the aluminosilicate sources used in SBTE, focusing primarily on the emitter surface coating quality and manufacturing techniques. We initially chose to purchase commercially availab' sources for our $\mathrm{Cs}^{+}$beam. We then found that in order to obtain the desired current, we must operate the sources at very close to the maximum heater power they could tolerate. As a result we burned out two heater filaments. In addition, the commercial coating of Cs-doped aluminosilicate material was insufficient for our use, because after 2-6 weeks in service (varying from source to source) the $\mathrm{Cs}^{+}$current became seriously diminished.

\section{E.1 Source Recoating Procedure}

The source bodies were satisfactory, although they - suld just barely maintain the temperature required to emit the desired current. After replacing several sources because of cesium depletion, we decided to recoat the emitter surfaces ourselves with an aluminosilicate mixture. Dr. S. S. Rosenblum mixed alumina, silica, and $\mathrm{Cs}_{2} \mathrm{CO}_{3}$ in the proper stoichiometric proportions for the $\beta$-eucryptite zeolite, and fused the material in an inert gas furnace. After he had re-pulverized the mixture, we applied it to the source bodies and found techniques lescribed in the following for obtaining suitable adhesion between the aluminosilicate and the substrate.

We constructed a vacuum furnace of simple design (a cylindrical winding of 0.03-inch tantalum wire large enough to encircle the source-about 2 inches in diameter-and about 4 inches tall, supported by high-grade alumina tubing with slots cut for support of the hot wire, and surrounded by about 10 layers of 0.002 -inch molybdenum foil to serve as a heat shield, all mountable in a small yacuum chamber that was available) and baked the spent sources at $1700^{\circ} \mathrm{C}$ for 10 minutes. The overall cycle time was about 30 minutes to 
$1700^{\circ} \mathrm{C}, 10$ minules at $1700^{\circ} \mathrm{C}$, and then power off. We applied the powdered aluminosilicate (200 mesh) to the emitter surface in slurry form, using either distilled water or methanol, to a depth of about 0.010-0.020 inch. After pumping the liquid away in the vacuum for several hours, we raised the temperaiure as rapidly as possible, consistent with keeping the pressure reading below $10^{-4}$ Torr. A substantial amount of vapor was evolved, possibly $\mathrm{CO}_{2}$ entrained in the aluminosilicate in addition to the remaining carrier fluid for the coating. The furnace cycle was similar to the bake cycle for cleaning the sources.

The stack of heat shields at the top of the furnace had a hole through it, permitting optical measurements of the temperature. The pyrometer filters and optics permitted me to observe when the aluminosilicate inixture began to fuse. After the coating began to fuse visibly, we quenched the furnace by venting it with argon gas and turning off the heater power. The argon quench was an attempt to collapse small bubbles (of $\mathrm{CO}_{2}$ ?) of 0.01-0.02-inch diameter in the molten aluminosilicate before the material solidified.

The new coatings were sometimes too thick; and the thermal conductivity was apprarently too low to allow the emitter surface to reach the required temperature. The pyrometer-measured temperature of the emitter surface was below the temperature of the commercially coated sources by about $50^{\circ} \mathrm{C}$. After we ground the coating down to a thickness of about 0.010 inch, the temperature returned to its former value and the $\mathrm{Cs}^{+}$current rose to useful levels. The bubbles mentioned above were visible in the surface of the aluminosilicate, and can cause inhomogeneous emission of the ion beam. Transverse fields near the surface capable of degrading the emittance by a large factor over the intrinsic thermal emittance can easily result. The test stand geometry was not the same as the SBTE gun, so final tests had to be done in the actual SBTE gun. 


\section{E.2 Performance}

\section{E.2.1 Current}

The new coatings provided marginally higher bearn current than the original sources. However, the major gain was in longevity. The first of the two recozted sources we used lasted for nine months of regular use, and the second lasted for over a year. The source presently in use is a test formulation made by A.A. Warwick during his research on source fabrication for the MBE-4 accelerator experiment [51].

\section{E.2.2 Source Emittance}

The lower bound $\epsilon_{\min }$ on the normalized source emittance ( $4 \epsilon_{\mathrm{KM}}$ ) is given by the source radius, $R_{\mathrm{S}}$, and the mean thermal energy $\frac{k T}{2}$ in each transverse plane as

$$
\epsilon_{\min }=2 R_{s} \sqrt{\frac{\mathrm{kT}}{\mathrm{mc}^{2}}}=0.23 \times 10^{-7} \pi \text { meter radian }
$$

This is about a factor of 5 below the measured beam emittance, and indicates that there is a significant perturbation to the transverse velocity spread of the particles even before they exit the gun. Additional perturbations to the emittance of the bearn, due to passage through grids at the exit of the gun, are calculated and compared with measurements in Appendix C.

\section{E.2.3 Beam Purity}

When our in-house sources were first installed, the $\mathrm{Cs}^{+}$beam was very pure. As the sources aged, they all began to yield a small proportion of light ions along with the bulk $\mathrm{Cs}^{+}$beam. The appearance of the light ions is accompanied by a decrease in the cesium component of the beam. When the light-ion fraction grew to about $5 \%$, the source was no longer deemed 
satisfactory, and it was replaced at that time.

The light ions separate themselves from each other and from the cesium component by time-of-flight in short-pulse beam operation. This effect was used to analyze the mass spectrum of the light ions. We did this after the useful life of the first recoated source we used, during the initial operation of the injector in its shortened configuration. With more current being drawn from the injector, the light ion fraction had risen to nearly $20 \%$. We took this opportunity to measure the relative proportion of various mass numbers in the beam, rather than replace the source immediately.

Near the source, before the light ions separate from the bulk cesium beam, they experience the fields of the cesium component. Because static electric fields function as energy separators, rather than momentum separators as do magnetic fields, the light ions respond exactly as do the cesium ions to the self- and external fields. Later in the lattice, when they leave the bulk $\mathrm{Cs}^{+}$ beam and its space-charge fields, they are mismatched, and a large proportion is lost to the walls. About one-third of the light ion component was lost after separating from the bulk $\mathrm{Cs}^{+}$beam, based on Faraday cup measurements along the lattice.

As the light ion components separated in time, downward steps in the trailing edge of the current pulse, measured at the end of the transport line, allowed the masses to be calculated from time-of-light. This is similar the trailing step visible on the current pulses in Fig. 5.3. By far the largest lightion component was at $A=28$ (calculated with respect to the ${ }^{133} \mathrm{Cs}$ tirne of flight). Masses of other trace components were calculated with respect to the $A=28$ component. This main component $(A=28)$ accounted for almost $90 \%$ of the total light ions. Both silicon $(A=28)$ and aluminum $(A=27)$ are components of the emitter, and metallic alvminum is the material forming 
A I $\quad I(\mathrm{~mA}) \quad \begin{gathered}\% \text { of } \\ \text { light ions }\end{gathered}$ Possible species (based on $A / q$ )

\begin{tabular}{|c|c|c|c|}
\hline 16 & 0.03 & 1.5 & $\mathrm{O}^{+}(16)$ \\
\hline 28 & 1.7 & 88. & $\mathrm{Si}^{+}(28)_{3} \mathrm{Al}^{+}(27)$ \\
\hline 31 & 0.08 & 4. & $\mathrm{O}_{2}^{+}(32)$ \\
\hline 39 & 0.05 & 3. & $\mathrm{~K}^{+}(40)$ \\
\hline 44 & 0.04 & 2. & $\mathrm{SiO}^{+}(44), \mathrm{AlO}^{+}(43)$ \\
\hline $48-49$ & 0.01 & 0.5 & $\mathrm{Al}_{2} \mathrm{O}_{3}^{+2}(51)$ \\
\hline 57 & 0.01 & 0.5 & $\mathrm{AlO}_{2}^{+}(57), \mathrm{SiO}_{2}^{+}(58)$ \\
\hline 74 & 0.01 & 0.5 & $\mathrm{Al}_{2} \mathrm{O}^{+}(70), \mathrm{AlO}_{3}^{+}(75)$ \\
\hline 86 & 0.01 & 0.5 & $\mathrm{Al}_{2} \mathrm{O}_{2}^{+}(86)$ \\
\hline
\end{tabular}

Table E.1: Distribution of light ion impurities

most of the gun structure, and both are probably present in this mass peak. Other ion masses obtained assuming the mass of the major component to be 28 were consistent with a number of dissociation products of $\mathrm{Al}_{2} \mathrm{O}_{3}$ and $\mathrm{SiO}_{2}$. The mass determination could be in error by \pm 2 amu. Results are shown in Table E.1. We also note that there was a time delay of about 200-300 ns after the $\mathrm{Cs}^{+}$emission began before the light ion emission began. By applying very short pulses to the gun, we were able to obtain $\mathrm{Cs}^{+}$currents of more than $75 \%$ of the steady-state level with no measurable light ion component. 


\section{Bibliography}

[1] John Nuckolls. Laser Fusion Overview. Technical Report UCRL 77725, Lawrence Livermore Laboratory, May 197C.

[2] A. W. Maschke. Initiation of Thermonuclear Detonations. Technical Report BNL 19008, Brookhaven National Laboratory, June 1974.

[3] L. C. Teng. Technical Report ANLAD 59, Argonne National Laboratory, Argonne, Illinois, February 1963.

[4] L. J. Laslett. On Intensity Limitations Imposed by Transverse SpaceCharge Effects in Circular Particle Accelerators. In Proceedings of the 1969 Summer Study on Storage Rings, Accelerators, and Experimentation at Super-High Energies, pages 324-367, Brookhaven National Laboratory, 1963. BNL 7534.

[5] E. J. N. Wilson. The Importance of Non-linearities in Modern Proton Linear Accerators. In Proceedings of the First Course of the International School of Particle Accelerators, pages 111-138, CERN-77-13, 1977.

(6) Denis Keefe. Inertial Confinement Fusion. Annual Review of Nuclear and Particle Science, 32:391-441, 1982.

[7] David L. Judd. Phase-Space Constraints on Induction-Linac HeavyIon Inertial-Fusion Igniters. Technical Report LBL-14038, Lawrence Berkeley Laboratory, January 1982.

[8] J. D. Lawson. Proc. Phys. Soc. (London), B70:2-10, 1957.

[9] George Schmidt. Physics of High Temperature Plasmas. Acadenic Press, New York, second edition, 1979.

[10] U. von Mollendorff. The HIBALL Study. In Proceedings of the 1984 INS Symposium on Heavy Ion Accelerators and their Applications to Inertial Fusion, pages 155-167, Institute for Nuclear Study, Tokyo, January 1984.

[11] T. Yamaki. A Design Study of HIF, HIBLIC-I. In Proceedings of the 1981 INS Symposium on Heavy Ion Accelerators and their Applications to Inertial Fusion, pages 141-154, Institute for Nuclear Study, Tokyo, January 1984. 
[12] M. J. Monsler, et al. An Overview of Inertial Fusion Reactor Design. Nuclear T'echnology/Fusion, 1:302-358, July 1981.

[13] Thor $O$. Bohachevsky. Models and Analyses for Inertial Confinement $F_{u-}$ sion Reactor Studies. Technical Report LA-8557, Los Alamos Scientific Laboratory, May 1981.

(14) P. Lapostolle. Possible Emittance Increase Through Filamentation Due to Space Charge in Continuous Beams. In Proceedings of the 1911 Particle Accelerator Conference, pages 1101-1104, IEEE Transactions on Nuclear Science, NS-18, 1971.

[15] R. L. Gluckstern. Oscillation Modes in Two Dimensional Beams. In Proceedings of the 1970 Proton Linear Accelerator Conference, pages 811822, FNAL, Batavia, Illinois, 1970.

[16] L. Smith, S. Chattopadhyay, I. Hofmann, and L. J. Laslett. Technical Report HIFAN-13-15, unpubl., Lawrence Berkeley Laboratory, 1977.

[17] I. Hofmann. Negative Energy Oscillations and Instability of Intense Beams. Particle Accelerators, 10:253-258, 1980.

[18] George K. Brewer. Some Characteristics of a Magnetically Focused Electren Beam. Journal of Applied Physics, 30(7):1022-1038, 1959.

[19] L. Brillouin. A Theorem of Larmor and its Importance for Electrons in Magnetic Fields. Physical Review, 67:260-266, 1945.

[20] I. Hofmann, L. J. Laslett, L. Smith, and I. Haber. Stability of the Kapchinskij-Vladirmiskij (K-V) Distribution in Long Periodic Transport Systems. Particle Accelerators, 13:145, 1983.

[21] Han S. Uhm and Ronald C. Davidson. Stability Properties of Intense Nonneutral Ion Beams for Keavy Ion Fusion. Technical Report PFCJA-79-20, Massachusetts Institute of Technology Plasma Fusion Center, December 1979.

[22] A. W. Maschke. Heavy Ion Space Charge Limits. Technical Report BNL 20297, Brookhaven National Laboratory, July 1975.

[23] J. Klabunde, P. Spādtke, and A. Schōnlein. High Current Beam Transport Experiments at GSI. In Proceedings of the 1985 Particle Accelerator Conference (Vancouver), IEEE Transactions on Nuclear Science, NS-32, pages 2462-2464, 1985. 
[24] J. MaAdoo, et al. Electron Beam Transport and Emittance Measurements in a Long Periodic Solenoid Channel. In Proctedings of the 1985 Particle Accelerator Conference (Vancouver), IEEE Transactions on Nuclear Science, NS-32, pages 2632-2634, 1985.

[25] M. G. Tiefenback and D. Keefe. Measurements of Stability Limits for a Space-Charge-Dominated Ion Beam in a Long A. G. Transport Channel. In Proceedings of the 1985 Particle Accelerator Conference, IEEE Transactions on Nuclear Science, NS-32, pages 2483-2485, 1985. also LBL-19647.

[26] E. D. Courant and H. S. Snyder. Theory of the Alternating Gradient Synchrotron. Annals of Physics, 3:1-48, 1958.

[27] Henri Brück. Circular Particle Accelerators (transl.). Presses Universitaires de France, 1966. (transl. pub. by Los Alamos Scientific Laboratory, LA-TR-72-10), 1972.

[28] J. D. Lawson. Space-Charge Optics. In A. Septier, editor, Applied Charged Particle Optics, Part $C$, pages 1-48, Academic Press, New York, N. Y., 1983.

[29] Ronald C. Davidson. Theory of Nonneutral Plasmas. W. A. Benjamin, Inc., Reading, Massachusetts, 1974.

[30] I. V. Kapchinskij and V. V. Vladirmiskij. Limitations of Proton Beam Current in a Strong Focusing Linear Accelerator Associated with the Beam Space Charge. In Proceedings of the Second International Conference on High-Energy Accelerators and Instrumentation, pages 274-288, CERN, 1959.

[31] Frank J. Sacherer. RMS Envelope Equations with Space Charge. In Proceedings of the 1971 Particle Accelerator Conference, pages 11051107, IEEE Transactions on Nuclear Science, NS-18, 1971.

[32] R. K. Feeney, William E. Sayle, II, and J. W. Hooper. Aluminosilicate sources of positive ions for use in collision experiments. Reviews of Scientific Instruments, 47(8):9004-967, August 1976.

[33] A. N. Pargellis and M. Seidl. Thermionic emission of alkali ions from zeolites. Journal of Applied Physics, 49(9):4933-4938, September 1978. 
[34] W. B. Herrmannsfeldt. SLAC Electron Optics Program. Technical Report SLAC report 166, Stanford Linear Accelerator Center, September 1973.

[35] Michael Lampel and Michael Tiefenback. An applied voltage to eliminate current transients in a one-dimensional diode. Applied Physics Letters, 43(1):57-58, July 1983.

[36] A. W. Maschke, J. Brodowski, and E. Meier. Space Charge Limits in ESQ Transport Systems. In Proceedings of the 1989 Particle Accelerator Conference (Santa $\mathrm{Fe}$ ), IEEE Transactions on Nuclear Science, NS-30 (4), p. 2558, 1983.

[37] Andris Faltens. Private communication, May 1986.

[38] Charles Kim. Single Beam Transport Experiment Reference Parameters. Technical Report HIFAN-160, Lawrence Berkeley Laboratory, March 1981. Unpublished.

[39] Andris Faltens and S. S. Rosenblum. Electrostatic Energy Analyzer (EEA) for HIFAR. Technical Report HIFAR-17, unpublished, Lawrence Berkeley Laboratory, Berkeley, California, 1985.

[40] J. Strückmeier and M. Reiser. Theoretical Studies of Envelope Oscillations and Instabilities of Mismatched Intense Charged-Particle Beams in Periodic Focusing Channels. Particle Accelerators, 15:227-260, 1984.

[41] C. M. Celata, I. Haber, L. J. Laslett, L. Smith, and M. G. Tiefenback. The Effect of Induced Charge at Boundaries on Transverse Dynamics of a Space-Charge-Dominated Beam. In Proceedings of the 1985 Particle Accelerator Conference (Vaneouver), IEEE Transactions on Nuclear Science, NS-32, pages 2480-2482, 1985. also LBL-19485.

[42] J. Strūckmeier, J. Klabunde, and M. Reiser. On the Stability and Emittance Growth of Different Particle Phase-Space Distributions in a Long Magnetic Quadrupole Channel. Particle Accelerators, 15:47-65, 1984.

[43] T. P. Wangler, K. R. Crandall, R. S. Mills, and M. Reiser. Relation Between Field Energy and RMS Emittance in Intense Particle Beams. In Proceedings of the 1985 Particle Accelerator Conference (Vancouver), IEEE Transactions on Nuclear Science, NS-32, pages 2196-2200, 1985. 